Andreas Boesch

\title{
Construction and Analysis of a Microwave-induced Plasma Lamp For Precision Spectroscopy
}

Dissertation

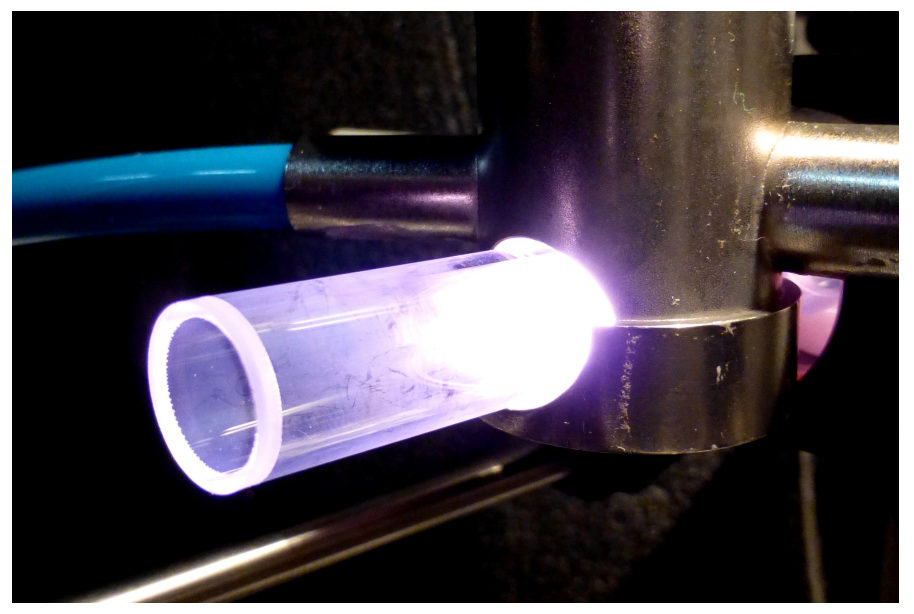





\title{
Construction and Analysis of a Microwave-induced Plasma Lamp for Precision Spectroscopy
}

\author{
Dissertation \\ zur Erlangung des mathematisch-naturwissenschaftlichen Doktorgrades \\ "Doctor rerum naturalium" \\ der Georg-August-Universität Göttingen \\ im Promotionsprogramm ProPhys \\ der Georg-August University School of Science (GAUSS)
}

vorgelegt von

Andreas Boesch

aus Flensburg

Göttingen, 2016 


\section{Betreuungsausschuss}

Prof. Dr. Ansgar Reiners, Sonnenphysik und stellare Astrophysik, Institut für Astrophysik, Georg-August-Universität Göttingen

Prof. Dr. Stefan Dreizler, Sonnenphysik und stellare Astrophysik, Institut für Astrophysik, Georg-August-Universität Göttingen

$\underline{\text { Mitglieder der Prüfungskommission }}$

Referent: Prof. Dr. Ansgar Reiners, Sonnenphysik und stellare Astrophysik, Institut für Astrophysik, Georg-August-Universität Göttingen

Korreferent: Prof. Dr. Thomas Giesen, Laborastrophysik, Universität Kassel

Weitere Mitglieder der Prüfungskommission:

Prof. Dr. Wolfram Kollatschny, Extragalaktische Astrophysik, Institut für Astrophysik, Georg-August-Universität Göttingen

Prof. Dr. Wolfgang Glatzel, Sonnenphysik und stellare Astrophysik, Institut für Astrophysik, Georg-August-Universität Göttingen

Prof. Dr. Claus Ropers, Nano-Optik und ultraschnelle Dynamik, IV. Physikalisches Institut, Georg-August-Universität Göttingen

Prof. Dr. Manfred Schüssler, Sonne und Heliosphäre, Max-Planck-Institut für Sonnensystemforschung 


\section{Abstract}

Spectroscopy is one of the most powerful techniques to analyze light of astronomical objects. New instrumentation strives to extend high-precision spectroscopy from the optical to the near-infrared spectral range. This development is driven by research on cool low-mass stars and the search for low-mass extrasolar planets with the radial velocity technique. A crucial requirement for precise spectroscopic measurements is the wavelength calibration that maps the pixels of a spectrograph's detector to the corresponding wavelengths. In the optical, hollow cathode lamps and gas absorption cells are established wavelength calibrators, but these techniques cannot be readily transferred to the near-infrared because they do not provide enough spectral lines over a broad spectral range. Many spectral lines in the near-infrared are present in discharge spectra of molecules, such as nitrogen or CN. In this thesis, I investigate whether discharge spectra of these two molecules are potential wavelength references for astrophysical spectrographs.

An experimental setup with a microwave-induced plasma lamp has been constructed. Characteristics of this lamp are the electrodeless design and the relatively inexpensive equipment. The plasma is sustained within a sealed glass tube so that measurements can be repeated with the same gas mixture and gas pressure. In addition, the sealed cell allows for a compact setup with no requirements for gas supply during operation. Spectra of different gas discharges, using two sealed gas cells, are recorded with a high-resolution Fourier transform spectrometer. One cell is filled with pure nitrogen gas, while the second cell is filled with a gas mixture producing emission from $\mathrm{CN}$ molecules. In the context of wavelength calibration, the analysis of the spectra focuses on line density, relative line intensities, wavelength stability and aging behavior.

The nitrogen discharge provides a spectrum with densely-spaced emission lines over the whole spectral range $4500-11000 \mathrm{~cm}^{-1}(0.9-2.2 \mu \mathrm{m})$. In the spectrum of the second discharge cell, about 4500 lines of $\mathrm{CN}$ and about 26000 lines of molecular nitrogen are detected (4500-10000 $\mathrm{cm}^{-1}$ ). Both species combined provide about four times more lines as uranium from hollow cathode lamps in this spectral range. The wavelength stability of the spectra is measured with a precision of about $1 \mathrm{~m} / \mathrm{s}$ over 24 hours. These properties make the microwave-induced plasma lamp an interesting candidate for wavelength calibration of future high-resolution spectrographs. However, the number of spectral lines usable for calibration will be reduced due to inhomogeneities regarding line spacing and line intensities, depending on the characteristics of a spectrograph (e.g., resolution and detector response). The demonstrated operational time of a single gas cell is about 180 hours, which is about 4-6 times shorter than typical lifetimes of hollow cathode lamps.

Application of the microwave-induced plasma lamp for astrophysics is not limited to the task of wavelength calibration. An example is the laboratory study of molecular spectra 
which can be used to analyze magnetic fields in cool stars. First measurements with the discharge lamp operated in a magnetic field are presented. A magnetic flux density of $B=(0.130 \pm 0.003) \mathrm{T}$ is determined using the Zeeman-splitting of three argon lines. Measurements of molecular species, such as $\mathrm{CN}$, in a calibrated field could be used in the future to identify magnetically-sensitive lines and to determine Landé factors. 


\section{Zusammenfassung}

Die Spektroskopie ist eine der leistungsfähigsten Techniken um das Licht von astronomischen Objekten zu analysieren. Neue Instrumente sollen hochpräzise Spektroskopie nicht nur im optischen, sondern auch im nah-infraroten Spektralbereich ermöglichen. Diese Entwicklung wird u.a. durch die Untersuchung von kalten Sternen mit geringer Masse und durch die Suche nach extrasolaren Planeten mithilfe der Radialgeschwindigkeitsmethode angetrieben. Eine entscheidende Voraussetzung für präzise Spektroskopie ist die Wellenlängenkalibrierung, die den Pixeln des Detektors die entsprechenden Wellenlängen zuordnet. Hohlkathodenlampen und Gasabsorptionszellen sind etablierte Methoden zur Kalibrierung im optischen Spektralbereich. Diese Techniken können jedoch nicht ohne Weiteres im nahen Infrarot angewendet werden, weil sie nicht genug Spektrallinien über einen weiten Wellenlängenbereich aufweisen. Emissionsspektren von Molekülen, wie z.B. Stickstoff oder CN, besitzen hingegen viele Spektrallinien im nahen Infrarot. In dieser Arbeit wird untersucht, ob sich Emissionsspektren von diesen beiden Molekülen zur Wellenlängenkalibrierung von Spektrographen in der Astrophysik eignen.

Ein Versuchsaufbau mit einer mikrowelleninduzierten Plasmalampe wurde konstruiert. Eigenschaften dieser Lampe sind der elektrodenlose Betrieb und das relativ kostengünstige Equipment. Das Plasma wird innerhalb eines geschlossenen Glasrohres erzeugt, sodass Messungen mit gleichbleibenden Gasgemischen und Drücken durchgeführt werden können. Diese geschlossene Gaszelle ermöglicht darüber hinaus einen kompakten Versuchsaufbau, der während des Betriebs unabhängig von einer Gasversorgung ist. Mit einem hochauflösenden Fourier-Transform-Spektrographen werden Spektren von verschiedenen Gasentladungen aufgenommen. Dabei werden zwei verschiedene Gaszellen verwendet. Eine Zelle ist mit reinem Stickstoff gefüllt, während die andere Zelle mit einem Gasgemisch gefüllt ist, welches Emission von CN Molekülen erzeugt. Die Spektren werden hinsichtlich der Liniendichte, relativen Linienintensitäten, Wellenlängenstabilität und des Alterungsprozesses untersucht.

Das Stickstoffplasma liefert ein dichtes Emissionsspektrum mit Spektrallinien im gesamten Spektralbereich 4500-11000 $\mathrm{cm}^{-1}(0.9-2.2 \mu \mathrm{m})$. Im Emissionsspektrum der zweiten Gaszelle werden ca. 4500 Linien von CN und ca. 26000 Linien von molekularem Stickstoff detektiert (4500-10000 $\mathrm{cm}^{-1}$ ). Beide Spezies zusammen liefern ungefähr vier Mal mehr Linien als Uran in Hohlkathodenlampen in diesem Spektralbereich. Die Wellenlängenstabilität der Spektren kann mit einer Präzision von ungefähr $1 \mathrm{~m} / \mathrm{s}$ über 24 Stunden gemessen werden. Diese Eigenschaften machen die mikrowelleninduzierte Plasmalampe zu einer interessanten Alternative für die Kalibrierung von zukünftigen hochauflösenden Spektrographen. Die unterschiedlichen Linienintensitäten und Abstände zwischen den Linien führen jedoch dazu, dass nicht alle Spektrallinien für eine Kalibrierung zur Verfügung stehen werden, abhängig von den konkreten Eigenschaften eines Spektrographen (z.B. Auflösung und 
Detektorempfindlichkeit). Die demonstrierte Betriebsdauer einer Gaszelle beträgt ca. 180 Stunden und ist damit ungefähr 4-6 Mal kürzer als die typische Lebensdauer von Hohlkathodenlampen.

Die Verwendung der mikrowelleninduzierten Plasmalampe für die Astrophysik ist nicht auf Wellenlängenkalibrierung beschränkt. Ein weiteres Beispiel ist die Untersuchung von Molekülspektren im Labor, welche verwendet werden können um Magnetfelder in kalten Sternen zu analysieren. Es werden erste Messungen mit der Plasmalampe in einem Magnetfeld präsentiert. Eine magnetische Flussdichte von $(0.130 \pm 0.003) \mathrm{T}$ kann über die Zeeman-Aufspaltung von drei Argon-Linien bestimmt werden. Die Analyse von Molekülen, wie z.B. CN, in einem kalibrierten Magnetfeld könnte in Zukunft dafür verwendet werden, um magnetisch sensitive Linien zu identifizieren und Landé-Faktoren zu bestimmen. 


\section{Contents}

1 Introduction $\quad 1$

1.1 Precision spectroscopy, radial velocities and exoplanets . . . . . . . . . . . . 1

1.2 Wavelength calibration of spectrographs . . . . . . . . . . . . . . 4

1.2.1 Telluric lines and gas absorption cells . . . . . . . . . . . . . 4

1.2.2 Hollow cathode lamps . . . . . . . . . . . . . . . . . 6

1.2.3 Fabry-Perot etalons and laser frequency combs . . . . . . . . . . 7

1.3 Why a microwave-induced plasma lamp? . . . . . . . . . . . . . . . 9

1.4 Outline of the thesis . . . . . . . . . . . . . . . . 11

2 The radial velocity technique 13

2.1 Introduction . . . . . . . . . . . . . . . . . . . . . . . . . . 13

2.2 The radial velocity equation . . . . . . . . . . . . . . . . . 14

2.2.1 Characteristics of an elliptical orbit . . . . . . . . . . . . . . . 14

2.2 .2 Derivation of the radial velocity equation . . . . . . . . . 15

2.2 .3 The radial velocity semi-amplitude . . . . . . . . . . . . 18

2.3 Radial velocity induced wavelength shifts in spectra . . . . . . . . . . 20

2.4 Measuring relative radial velocities from spectra . . . . . . . . . . . . . . 21

2.4.1 Cross-correlation and forward-modeling . . . . . . . . . . . . 21

2.4.2 Simultaneous RV measurements of two combined sources . . . . . . 22

2.5 Radial velocity information content of a spectrum . . . . . . . . . . . . 27

3 Fourier transform spectroscopy 31

3.1 Introduction . . . . . . . . . . . . . . . . . . 31

3.2 Principles of Fourier transform spectroscopy . . . . . . . . . . . . . . . 32

3.2 .1 The ideal instrument . . . . . . . . . . . . . . . . 32

3.2.2 Limited optical path difference and apodization . . . . . . . . . . . 34

3.2 .3 Finite size of entrance aperture . . . . . . . . . . . . . . 37

3.2 .4 Phase shift . . . . . . . . . . . . . . . . 40

3.3 The Bruker IFS $125 \mathrm{HR} \ldots \ldots \ldots \ldots \ldots$. . . . . . . . . . . 40

3.4 Analysis of FTS spectra . . . . . . . . . . . . . . . . . . 44

3.4.1 Determination of noise level . . . . . . . . . . . . . . . 44

3.4.2 A multi-profile fit algorithm for emission line spectra . . . . . . . . . 46

4 The microwave-induced plasma lamp $\quad 51$

4.1 Introduction . . . . . . . . . . . . . . . . . . 51

4.2 Microwave-induced plasmas . . . . . . . . . . . . . . . . 52

4.3 Components of the experimental setup . . . . . . . . . . . . . 54

4.3 .1 Overview .......................... 54 
4.3 .2 Microwave generator . . . . . . . . . . . . . . . 56

4.3 .3 Microwave cavity . . . . . . . . . . . . . . 5 56

4.3 .4 Gas cells . . . . . . . . . . . . . . . . . 57

4.3.5 Further equipment . . . . . . . . . . . . . . . . . 59

4.4 How to operate the discharge lamp . . . . . . . . . . . . . . . 59

5 Near-infrared wavelength calibration with molecular discharge spectra $\quad \mathbf{6 1}$

5.1 Introduction . . . . . . . . . . . . . . . . . 61

5.2 Spectral characterization of discharge gas cells . . . . . . . . . . . . . . 62

5.2 .1 Summary of measurements and data analysis . . . . . . . . . . 62

5.2 .2 The $\mathrm{N}_{2}$ discharge cell . . . . . . . . . . . . . . . . 68

5.2 .3 The CN discharge cell . . . . . . . . . . . . . . . 78

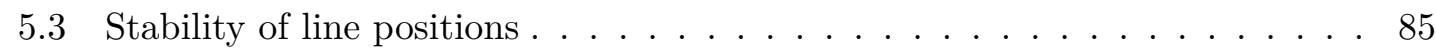

5.3 .1 Measurement procedure . . . . . . . . . . . . . . 85

5.3.2 Algorithm for measuring relative radial velocity shifts . . . . . . . 86

5.3 .3 Limits on radial velocity precision . . . . . . . . . . . . 8 87

5.3 .4 Radial velocity stability . . . . . . . . . . . . . . . . 89

5.4 Aging behavior . . . . . . . . . . . . . . . . . . . . 92

5.4 .1 Accelerated aging test . . . . . . . . . . . . . 92

5.4 .2 Discussion of aging behavior . . . . . . . . . . . . . . . 94

5.5 Measurements with CRIRES . . . . . . . . . . . . . . . . 95

6 First tests with the discharge lamp in a magnetic field $\quad 99$

6.1 Introduction and motivation . . . . . . . . . . . . . . . . . 99

6.2 Selection and installation of permanent magnets . . . . . . . . . . . . . 101

6.3 Calibration of the magnetic field with argon lines . . . . . . . . . . . . . . 104

6.4 Outlook . . . . . . . . . . . . . . . . . . . . . . 108

$\begin{array}{lll}7 & \text { Summary and discussion } & 111\end{array}$

7.1 Wavelength calibration . . . . . . . . . . . . . . . . 112

7.2 Outlook . . . . . . . . . . . . . . . . . . . 113

$\begin{array}{ll}\text { Bibliography } & 115\end{array}$ 


\section{Introduction}

\section{Contents}

1.1 Precision spectroscopy, radial velocities and exoplanets . . . 1

1.2 Wavelength calibration of spectrographs .......... 4

1.2.1 Telluric lines and gas absorption cells . . . . . . . . . . . 4

1.2.2 Hollow cathode lamps . . . . . . . . . . . . . . . 6

1.2.3 Fabry-Perot etalons and laser frequency combs . . . . . . . . . 7

1.3 Why a microwave-induced plasma lamp? . . . . . . . . . 9

1.4 Outline of the thesis . . . . . . . . . . . . 11

\subsection{Precision spectroscopy, radial velocities and exoplanets}

Almost all information from astronomical objects beyond Earth's atmosphere reach us in form of electromagnetic radiation. Spectroscopy is one of the most powerful techniques to analyze this radiation. It allows to study the intensities of a source at the different wavelengths that compose the emitted light. In general, spectroscopy is the study of the interaction of light with matter (Bernath, 2005, Sect. 1.1).

Astronomical spectroscopy has enabled physicists to infer many properties of distant objects (e.g., stars, galaxies, interstellar matter), such as their chemical composition, temperature, density, magnetic fields, and motion (e.g., Tennyson, 2011, Sect. 1.2). Most detailed information is obtained from high-resolution spectra that allow to identify and analyze individual spectroscopic features at a high precision in wavelength. An overview over the many research topics of precision spectroscopy in astronomy - from abundance measurements, probing fundamental constants, astroseismology to extrasolar planets - is given in Santos et al. (2008). Recent textbooks on astrophysical spectroscopy have been written, e.g., by Tennyson (2011) and Appenzeller (2013): the first book focuses on the analysis of atomic and molecular spectra, while the second one is devoted to observational and technical aspects. A comprehensive review of the historical evolution of astronomical spectroscopy has been given by Hearnshaw (2014). These days, one of the most recognized applications for high-resolution, high-precision spectroscopy is the radial velocity method used to detect and confirm planets outside the Solar System (Santos et al., 2008). The search for planets orbiting stars other than our Sun is a strong driver in the development of new instruments for precision spectroscopy.

The possibility of the existence of alien worlds outside our Solar System has been one of the oldest questions of humankind (compare, e.g., Perryman, 2011, Sect. 1.1). Only about 25 
years ago, with the first discoveries of extrasolar planets, could this question be answered in the affirmative. The first detection of two planetary bodies orbiting around a pulsar was published in the year 1992 by Wolszczan \& Frail, and the first detection of a planet in an orbit around a Sun-like star followed in 1995 by Mayor \& Queloz. Since then, a combination of scientific interest and public attention has turned exoplanet research into one of the most active fields in astrophysics. An extrasolar planet, or exoplanet, is a planet that orbits a star other than our Sun. The definition of a planet in our Solar System has been given in resolution B5 by the International Astronomical Union (IAU). However, the classification of a planet, and the derived classification of an exoplanet (compare the recommendation of the IAU's Working Group on Extrasolar Planets from 2003), is somewhat ambiguous as discussed by Perryman (2011, Sect. 1.5).

As of 11 November 2015, a total number of 1056 exoplanet detections have been published in refereed papers ${ }^{1}$. I show in Fig. 1.1 a histogram with the number of newly detected exoplanets for each year ${ }^{2}$. The colors of the histogram bars indicate the methods that were used for detection. A direct detection of an exoplanet is difficult, because planets are very faint compared to their host star. Therefore, different indirect techniques have been developed alongside the method of "direct imaging". These indirect techniques measure the effect of the gravitational influence of the planet on the host star, or the obscuration of a small part of the star's disk by the planet, or the distortion caused by the planet in the gravitational bending of light. A detailed description of the different detections methods (astrometry, pulsar timing, microlensing, direct imaging, transits, and radial velocity) is given in several textbooks, e.g., in Seager (2010), Perryman (2011) and Kitchin (2012). By the number of detections, radial velocity measurements and transit observations are the two most successful methods. The radial velocity technique is the only detection method based on high-resolution spectroscopy and it will serve as an application example of precision spectroscopy throughout this thesis. With this technique, astrophysicists determine the velocity of a star along the line of sight to an observer by measuring the wavelength shift of stellar absorption lines in a recorded spectrum. To find extrasolar planets, this technique uses the motion of the planet and its host star around their common center of mass. I will discuss the physical and mathematical foundations of the radial velocity method in detail in Chap. 2 (see Fig. 2.1 for an illustration of the concept). The first radial velocity detections were published by Mayor \& Queloz (1995) and Marcy \& Butler (1996).

The number of transiting exoplanets has increased strongly since the first detections with the space-based telescopes CoRoT and Kepler in the years 2007 and 2010 (Barge et al., 2008, Borucki et al., 2010, respectively). Transit observations deliver an even larger number of exoplanet candidates. However, exoplanet candidates from transits observations need follow-up observations by other techniques, e.g., radial velocity measurements, to confirm

\footnotetext{
${ }^{1} \mathrm{~A}$ list of all detected exoplanets can be found in the "The Extrasolar Planets Encyclopaedia" at www. exoplanet.eu.

${ }^{2}$ Only data since the year 1992 is shown in Fig. 1.1, although two earlier detections were listed in the database at the date of data retrieval. These two detections were however confirmed years later and are not included in the figure for clarity. Which exoplanet detection was the first is a matter of debate, but the discoveries mentioned in the text are widely excepted milestones. See also Perryman (2012) and Kitchin (2012).
} 


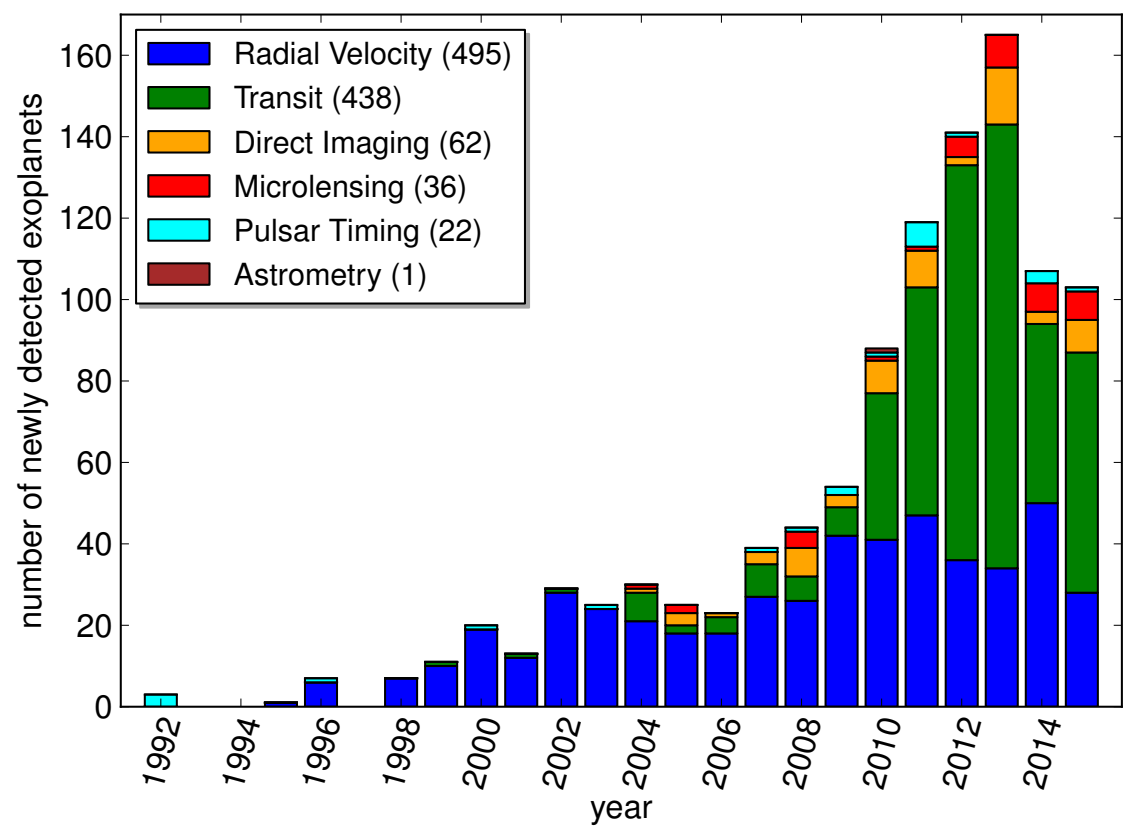

Figure 1.1: Number of detected and confirmed extrasolar planets per year (1992-2015). In the legend, the total number of detected exoplanets with each technique is given in parenthesis. The category "transit" includes transit timing variations, and the category "pulsar timing" includes detections based on orbit timing residuals. Created with data from www. exoplanet. eu retrieved on 11 November 2015.

the existence of the planet because the transit signal can be mimicked by different kinds of eclipsing systems (e.g., Bouchy \& Queloz, 2007).

An Earth-mass planet orbiting around a Sun-like star causes the star to move with a radial velocity of only about $10 \mathrm{~cm} / \mathrm{s}$ (Sect. 2.2.3). The precision to measure such small radial velocities cannot yet be accomplished by today's instruments. According to Appenzeller (2013), with present-day techniques radial velocity variations as small as about $1 \mathrm{~m} / \mathrm{s}$ can be observed. Therefore, a critical task to achieve more accurate radial velocity measurements is the development of new stable instruments, including precise wavelength references. In addition to increasing the accuracy of optical spectrographs, a different strategy is to observe stars with lower masses than the Sun. Because the gravitational pull by an exoplanet of a certain mass has a greater effect on a star with a lower mass, one expects a higher radial velocity signal (Sect. 2.2.3). Therefore, low-mass stars, such as M dwarfs, are natural targets for the search of low-mass planets. M dwarfs have a lower effective temperature compared to Sun-like stars and emit most of their light at wavelengths above $1 \mu \mathrm{m}$. They are best observed with spectrographs that are optimized for near-infrared wavelengths. Reiners et al. (2010) investigated the radial velocity precision that can be achieved for low-mass stars in the near-infrared. They conclude that measurements in the Y-, J- and H-bands result in a higher accuracy for M stars of spectral type later than M4.

For these reasons, $\mathrm{M}$ dwarfs are in the focus of future radial velocity surveys such as CARMENES which operates simultaneously with two spectrographs covering the optical 
(550-1050 nm) and the near-infrared (900-1700 nm) wavelength range (Quirrenbach et al., 2014). Other spectrograph projects in planning or under construction covering the nearinfrared include SPIRou, HZPF, CRIRES+, and E-ELT/HIRES (Thibault et al., 2012, Mahadevan et al., 2012, Follert et al., 2014, Zerbi et al., 2014, respectively). All these instruments require reliable wavelength calibration as discussed in the next section.

\subsection{Wavelength calibration of spectrographs}

A crucial task in precision spectroscopy is the wavelength calibration. Astrophysical spectrographs record the spectrum on a detector, e.g., a charge-coupled device (CCD) composed of a matrix of pixels. Each pixel at a position $(x, y)$ on the detector measures an intensity $I(x, y)$. The detector does however not provide information on the wavelength of the recorded light. The wavelength calibration assigns wavelengths $\lambda$ to the pixels of the spectrograph's detector, i.e., allows to transform $I(x, y)$ into $I(\lambda)$. This assignment is done using spectral lines with known wavelengths from a reference source. In other words, "The calibration is a process that establishes, under specified conditions, the relationship between the values indicated by the measuring system, and the corresponding values of a quantity realized by a reference standard or working standard" (Pepe \& Lovis, 2008, p. 378).

Different sources can serve as wavelength references for the calibration. In this section, I give an overview of the different types highlighting their advantages and disadvantages. Special attention is given to their performance in the near-infrared: sources for this spectral region are sparse, and new or improved techniques are required.

\subsubsection{Telluric lines and gas absorption cells}

In 1973, Griffin \& Griffin suggested the usage of absorption lines from the Earth's atmosphere (telluric lines) for the wavelength calibration to improve the accuracy in radial velocity measurements. At that time, the standard error for radial velocity measurements was at the level of $0.1-0.5 \mathrm{~km} / \mathrm{s}$. According to Griffin \& Griffin (1973), the dissimilarities in the illumination of the spectrograph by the reference lamp (e.g., a hollow cathode lamp as described below) and the star light limited the achievable accuracy. The usage of telluric lines could solve this problem, because the light of the reference spectrum would travel the same path as the star light. Further advantages claimed by Griffin \& Griffin (1973) include that the reference spectrum is superimposed on the stellar spectrum, and that this technique does not need maintenance because it is naturally available. They showed the feasibility of this calibration method by providing radial velocity measurements of the stars Arcturus and Procyon with standard errors of less than $0.03 \mathrm{~km} / \mathrm{s}$. However, Griffin \& Griffin (1973) also mentioned that calibration is only possible in wavelength regions where both stellar and telluric lines are present and of comparable strength.

In the near-infrared, spectra are dominated by atmospheric emission lines from hydroxyl $(\mathrm{OH})$ between $0.61 \mu \mathrm{m}$ and $2.62 \mu \mathrm{m}$ (Rousselot et al., 2000). Rousselot et al. (2000) provide 
a spectral line list of the night-sky $\mathrm{OH}$ airglow emission between $1 \mu \mathrm{m}$ and $2.25 \mu \mathrm{m}$ that can be used for wavelength calibration. The line list is based on medium resolution observations with the Infrared Spectrometer and Array Camera at the VLT telescope in Chile, and includes about 500 spectral lines. This atlas extends the previous work by Osterbrock et al. $(1996,1997)$ which covered the spectral range $0.519-1.060 \mu \mathrm{m}$, and was produced with data from the HIRES spectrograph at the 10-m Keck telescope in Hawaii. Abrams et al. (1994) carried out laboratory measurements of $\mathrm{OH}$ in the spectral range 1.1-5.4 $\mu \mathrm{m}$, derived molecular parameters and predicted line position in the optical down to around $500 \mathrm{~nm}$.

The CRIRES instrument at the VLT telescope is a high-resolution spectrograph operating in the infrared between $1 \mu \mathrm{m}$ and $5 \mu \mathrm{m}$ (Käufl et al., 2004). Based on experience made during the commissioning of CRIRES, Kerber et al. (2008) report that the number and distribution of night-sky emission lines is inadequate at a high resolving power of $R=\lambda / \Delta \lambda \approx 100000$. Only a few lines achieve a good signal-to-noise ratio at typical science exposure times. At wavelengths above $3 \mu \mathrm{m}$, the calibration with telluric absorption lines is possible, but the stability is limited because of bulk atmospheric motion (Kerber et al., 2008). Seifahrt \& Käufl (2008) carried out radial velocity measurements with CRIRES and investigated the stability of telluric lines at $4.1 \mu \mathrm{m}$ in a narrow spectral range of about $100 \mathrm{~nm}$. Over 4.5 hours, the line positions were stable to $10 \mathrm{~m} / \mathrm{s}$ measured relative to an $\mathrm{N}_{2} \mathrm{O}$ gas absorption cell. A similar precision on a time scale of one week with $\mathrm{CO}_{2}$ lines was reported by Figueira et al. (2010), again using the CRIRES instrument.

The properties of atmospheric features are always dependent on the telescope site and they vary with time. It would be desirable to maintain the calibration source under conditions that can be controlled by the observer, while at the same time keeping the advantages of the calibration method with telluric lines. This has been achieved by placing gas absorption cells in the light beam in front of the spectrograph. The absorption features of the captive gas are imprinted onto the stellar spectrum like telluric lines.

Being aware of the work by Griffin \& Griffin (1973) and the difficulties of the calibration with telluric lines, Campbell \& Walker (1979) suggested to position a cell filled with hydrogen flouride (HF) in front of the spectrograph's entrance. Hydrogen flouride was chosen for a couple of reasons, including the line separation of several angstroms in order to see unblended stellar lines in between. Later, Marcy \& Butler (1992) used an iodine $\left(\mathrm{I}_{2}\right)$ absorption cell for precision radial velocity measurements with the Hamilton Spectrograph at the Lick observatory. Marcy \& Butler (1992) highlight the advantages of $\mathrm{I}_{2}$ compared to HF: iodine has a strong line absorption coefficient and the gas cell only needs to be a few centimeters long to provide sufficiently deep absorption lines at a pressure much below atmospheric pressure, as compared to a cell length of one meter for HF; the wavelength coverage between $500 \mathrm{~nm}$ and $630 \mathrm{~nm}$ is much broader, as compared to only $10 \mathrm{~nm}$; iodine is chemically stable, only slightly corrosive and nonlethal. The iodine spectrum $F_{\mathrm{I}_{2}}$ is superimposed on the stellar spectrum $F_{\text {star }}$ and lines from both sources are blended. The combined spectrum is then convolved by the point spread function PSF of the instrument and the observed spectrum $F_{\text {obs }}$ is given by:

$$
F_{\mathrm{obs}}(\lambda) \approx\left[F_{\mathrm{star}}(\lambda) \cdot F_{\mathrm{I}_{2}}(\lambda+\Delta \lambda)\right] * P S F \quad .
$$


The stellar spectrum and the wavelength shift $\Delta \lambda$ relative to the iodine reference can be extracted through a deconvolution and modeling process (Marcy \& Butler, 1992, Lovis \& Fischer, 2010).

The calibration with $I_{2}$ is limited to optical wavelengths. Other gases that have been used for wavelength calibration at near-infrared wavelengths include nitrous oxide $\left(\mathrm{N}_{2} \mathrm{O}\right)$, ammonia $\left(\mathrm{NH}_{3}\right)$, and methane isotopologues (Käufl et al., 2004, Bean et al., 2010, AngladaEscudé et al., 2012, respectively). According to Bean et al. (2010), the ammonia cell allows to calibrate a spectral range of $36.4 \mathrm{~nm}$ at around $2.3 \mu \mathrm{m}$ with CRIRES. Cells filled with mixtures of different gases are being tested to extend the wavelength range that can be calibrated with a single setup. For example, the planned short-path gas cell for the upgraded CRIRES spectrograph (CRIRES+) contains acetylene, ammonia, and an isotopologue of methane (Seemann et al., 2014). A different combination of gases was investigated by Valdivielso et al. (2010) and a combination of four different commercially available gas cells was proposed by Mahadevan \& Ge (2009).

\subsubsection{Hollow cathode lamps}

Baranne et al. (1996) described a different calibration concept for the ELODIE spectrograph at the Observatoire de Haute-Provence. Instead of placing an absorption cell in front of the spectrograph, a hollow cathode lamp (thorium-argon) serves as a reference source. Optical fibers are used to overcome the problem with the illumination of the entrance slit and the collimator. A fiber acts as an image scrambler because of the multiple internal reflections. The resulting image at the output end of the fiber is essentially independent of illumination details at the fiber input. This makes illumination of the spectrograph isolated from telescope guiding errors or the alignment of the calibration sources (Heacox, 1986). The ELODIE spectrograph was used for the detection of the first extrasolar planet around a Sun-like star (51 Pegasi b; Mayor \& Queloz, 1995).

Hollow cathode lamps (HCL) provide a rich spectrum of sharp emission lines that can be used for wavelength calibration. The design and operation was summarized, e.g., by Kerber et al. (2007): a HCL contains a cylindrical cathode made from the material of interest, e.g., thorium or uranium, inside a glass enclosure filled with a buffer gas. Noble gases, usually neon or argon, are used as the buffer gas. An anode provides the potential to ionize the filling gas and the charged particles accelerate towards the cathode, where they sputter material into the discharge. The sputtered atoms collide with other particles, get excited, and emit light when they relax to the ground state. The spectrum of a HCL does not only contain emission lines from the cathode material, but also from the filling gas. The emission lines of the filling gas are often much stronger, and can cause saturation of the detector or scattered light making parts of the spectrum unusable (see, e.g., Fig. 1 in Redman et al., 2011 and Mahadevan \& Ge, 2009).

Several line lists for thorium-argon HCL have been compiled. Kerber et al. (2008) observed low-current ThAr lamps with a Fourier transform spectrometer and report 2400 lines that are usable for calibration in the wavelength range 900-4500 nm. Kerber et al. (2008) also give an overview of previous work on ThAr lamps in their Table 1, including the 
work by Palmer \& Engleman (1983), Hinkle et al. (2001), and Lovis \& Pepe (2007). The recent work by Redman et al. (2014) includes new observations of a ThAr lamp between $350 \mathrm{~nm}$ and $1175 \mathrm{~nm}$ which are combined with seven previously published thorium lists to optimize energy levels and calculate wavelengths for 19874 thorium lines between $250 \mathrm{~nm}$ and $5500 \mathrm{~nm}$. In contrast to the optical wavelength range, the density of thorium lines in the near-infrared is relatively low (e.g., Redman et al., 2012, Mahadevan \& Ge, 2009). An alternative is the usage of HCLs with uranium. Redman et al. (2011) compiled a line list for uranium from $850 \mathrm{~nm}$ to $4000 \mathrm{~nm}$ from observations of uranium-argon and uranium-neon HCLs. They report six times as many uranium lines as compared to thorium in the near-infrared.

Two main effects of aging have been reported for HCLs which limit the lifetime of the lamps (e.g., Kerber et al., 2007). First, the sputtering process removes material from the cathode which leads to an erosion of the cathode and to a change in the cathode's geometry. Second, the fill gas gets absorbed by the surfaces inside the lamp which leads to a decrease in gas pressure until the pressure is too low to sustain the discharge. Nave et al. (2012) report that the voltage increases over time for a HCL operated at a constant current, and suggest to use this quantity as a diagnostic tool of lamp aging.

\subsubsection{Fabry-Perot etalons and laser frequency combs}

The ideal calibrator for an astrophysical spectrograph provides equally-spaced spectral lines usable over the whole wavelength range of interest. In addition, all lines should be of similar intensity, and their position locked to a precise natural standard, such as an atomic energy transition (compare also Pepe \& Lovis, 2008). Compared to the previously mentioned calibration sources, Fabry-Perot etalons and laser frequency combs are relatively new techniques that are being developed for precise wavelength calibration.

The basic design of Fabry-Perot etalons consists of two parallel semitransparent mirrors separated by a fixed distance. The light of a continuum source is coupled into one end. The light that exits on the other end has a spectrum with emission peaks equally spaced in frequency (e.g., Demtröder, 2006, Sect. 10.4.1). The design of the Fabry-Perot can be customized to fit a certain spectral resolution (change mirror distance) and to cover a certain wavelength range (change mirror coating). The disadvantages of etalons are that their transmission signal drifts in wavelength under the influence of changing environmental conditions, and that the emission peaks are not automatically linked to a physical standard. Fabry-Perot etalons are used for the calibration of the CARMENES spectrograph in combination with HCLs. In preparation for this instrument, Schäfer \& Reiners (2012) analyzed the influence of the environmental conditions on the radial velocity precision of a Fabry-Perot etalon: in order to reach a precision of $10 \mathrm{~cm} / \mathrm{s}$, the pressure inside the Fabry-Perot needs to be stable to approximately $4 \cdot 10^{-3}$ mbar at a reduced pressure of 1 mbar. At a pressure of 1 mbar, the influence of temperature variations is also much less as at atmospheric pressure, but it still needs to be stabilized to $0.018 \mathrm{~K}$.

Laser frequency combs (LFC) work with pulsed lasers with a typical pulse repetition rate of $\nu_{\text {rep }}=250 \mathrm{MHz}$ (e.g., Udem et al., 2002, Wilken et al., 2010, 2012, Ycas et al., 2012). The 
laser light is sent through a non-linear fiber which broadens the spectrum to cover a larger wavelength range. The frequency spacing of the equidistant spectral lines corresponds to the repetition rate $\nu_{\text {rep }}$. The frequency of each line is given by $\nu_{n}=n \nu_{\text {rep }}+\nu_{0}$, where $n$ is an integer and $\nu_{0}$ is called the carrier-envelope offset frequency. This offset frequency is a result of a difference in phase and group velocity in the pulsed laser beam (see Fig. 1 in Murphy et al., 2007). To stabilize the laser output, the frequencies $\nu_{\text {rep }}$ and $\nu_{0}$ need to be detected and locked (e.g., Ycas et al., 2012). The repetition frequency can be directly measured with a fast photodiode. The offset frequency can be determined via heterodyne measurements from an octave-spanning spectrum (Udem et al., 2002): the beat signal of two signals with frequencies $2 \nu_{n}$ and $\nu_{2 n}$ is $\nu_{\text {beat }}=2\left(n \nu_{\text {rep }}+\nu_{0}\right)-\left(2 n \nu_{\text {rep }}+\nu_{0}\right)=\nu_{0}$. Both frequencies, $\nu_{\text {rep }}$ and $\nu_{0}$, can be locked to natural standards. One of the problems with current laser frequency combs is the small spacing between neighboring spectral lines, i.e., a small free spectral range. With a repetition rate of $\nu_{\text {rep }}=250 \mathrm{MHz}$, the free spectral range at wavelength $\lambda=600 \mathrm{~nm}$ is only $\Delta \lambda=0.0005 \mathrm{~nm}$. This cannot be resolved with astronomical spectrographs. For example, a high-resolution spectrograph with a resolving power of $R=\lambda / \Delta \lambda=100000$ resolves spectral features that are a minimum $\Delta \lambda=0.006 \mathrm{~nm}$ apart (at $\lambda=600 \mathrm{~nm}$ ). Therefore, the comb light is sent through Fabry-Perot cavities for filtering and to generate frequency combs with a larger free spectral range (e.g., Steinmetz et al., 2009). These Fabry-Perot cavities also need to be stabilized. Laser frequency combs are being tested with different spectrographs, e.g., at optical wavelengths with HAPRS at the 3.6-meter telescope in La Silla or at near-infrared wavelengths with the Pathfinder spectrograph at the 9.2-meter Hobby-Eberly telescope (Wilken et al., 2012, Ycas et al., 2012, respectively).

In summary, the established wavelength calibrators are iodine absorption cells and HCLs for the optical wavelength regime (e.g., Perryman, 2011). However, the transfer of these techniques into the near-infrared is not readily possible (e.g., Figueira et al., 2010, Mahadevan $\& \mathrm{Ge}, 2009)$. The line density of HCLs in the near-infrared is much lower than in the optical and strong lines from the fill gas contaminate the spectrum. For captive gas cells, there is no known gas which provides a high density of absorption lines over a wide range in the near-infrared. New techniques, such as Fabry-Perot etalons and laser frequency combs, are still under development and need to prove their long-time reliability. In addition, these new technologies still have shortcomings: Fabry-Perot etalons are susceptible to environmental conditions introducing wavelength drifts; laser frequency combs require a high financial investment and complex peak filtering to match the resolution of current astrophysical spectrographs.

Therefore, alternative calibration sources for the near-infrared should be investigated. In the next section, I argue why molecular emission spectra, as recorded from a microwaveinduced plasma lamp, have the potential to become viable wavelength references and should be investigated in this context. 


\subsection{Why a microwave-induced plasma lamp?}

The spectra of many molecular gas discharges consist of densely spaced emission lines in the near-infrared. An example for such a molecule is CN, a free radical that produces easily under conditions that provide donors of carbon and nitrogen (Ram et al., 2010). The rotation-vibrational bands of $\mathrm{CN}$ often appear as parasitic contributions in laboratory studies of other molecules, but were recognized as potential wavelength references for near-infrared spectrographs (A. Seifahrt and P. Bernath, private communication). The CN molecule has a prominent electronic transition in the visible to near-infrared, the "Red System" (A $\mathrm{A}^{2} \Pi-\mathrm{X}^{2} \Sigma^{+}$; e.g., Ram et al., 2010).

A histogram with the number of emission lines from the Red System of the CN molecule, as documented in the line list by Brooke et al. (2014), is shown in Fig. 1.2. Brooke et al. (2014) give observed and calculated values for the line positions. In the spectral range $4000-10000 \mathrm{~cm}^{-1}(1-2.5 \mathrm{\mu m})$ the line list contains about 27000 calculated transitions from over 60 vibrational bands. However, not all of these lines have been observed in experimental studies and cannot be expected to be usable for wavelength calibration. This number states an upper limit of the theoretically documented transitions and the corresponding line distribution is represented by the blue histogram. The green histogram uses data from the same line list but shows the distribution of observed lines from the three strong vibrational bands $0-0,0-1$, and $0-2\left(v^{\prime}-v^{\prime \prime}\right.$; vibrational quantum number in the upper state-vibrational quantum number in the lower state). The locations of the three bands are clearly visible with about 2500 lines. The distribution of $\mathrm{CN}$ emission lines that are present in a discharge can be expected to be somewhere between the blue and the green histogram, because lines from other vibrational bands should also be present in the spectrum. For comparison, the distributions of spectral lines from two species used in HCLs are also shown in Fig. 1.2. The line list of thorium compiled by Kerber et al. (2008) contains about 1200 lines in the spectral range $4000-10000 \mathrm{~cm}^{-1}$. This list was used to create the wavelength solution of the CRIRES spectrograph. A more recent atlas of thorium was compiled by Redman et al. (2014) including data from several previously published line lists (about 4000 lines). An alternative to thorium in HCLs is uranium which has a higher line density in the near-infrared. Redman et al. (2011) list about 7400 uranium lines. However, they operated a uranium-argon HCL at a current of $300 \mathrm{~mA}$ which is much higher than the typical operating currents for HCL used for wavelength calibration (about $10 \mathrm{~mA}$ ). It is important to note, that all line lists are based on observations which have been taken under different experimental conditions. The distributions shown in Fig. 1.2 are therefore not necessarily the intrinsic line densities, but nevertheless give an overview over the number of documented lines (compare also Fig. 6 in Redman et al., 2011).

Molecular gas discharges can be created with different experimental techniques. For example, Ram et al. (2010) list four different laboratory sources that have been used to record CN spectra. A microwave-induced plasma (MIP) lamp uses an electrodeless resonant cavity to create a gas discharge inside a non-conductive tube, e.g., a glass tube. The MIP lamp is characterized by comparatively low power, low running costs, and simple operation (Tschöpel, 1979, Sect. 8). To create CN in the discharge, different gases are mixed in the gas cell and excited in the MIP lamp. One of the components in this gas 


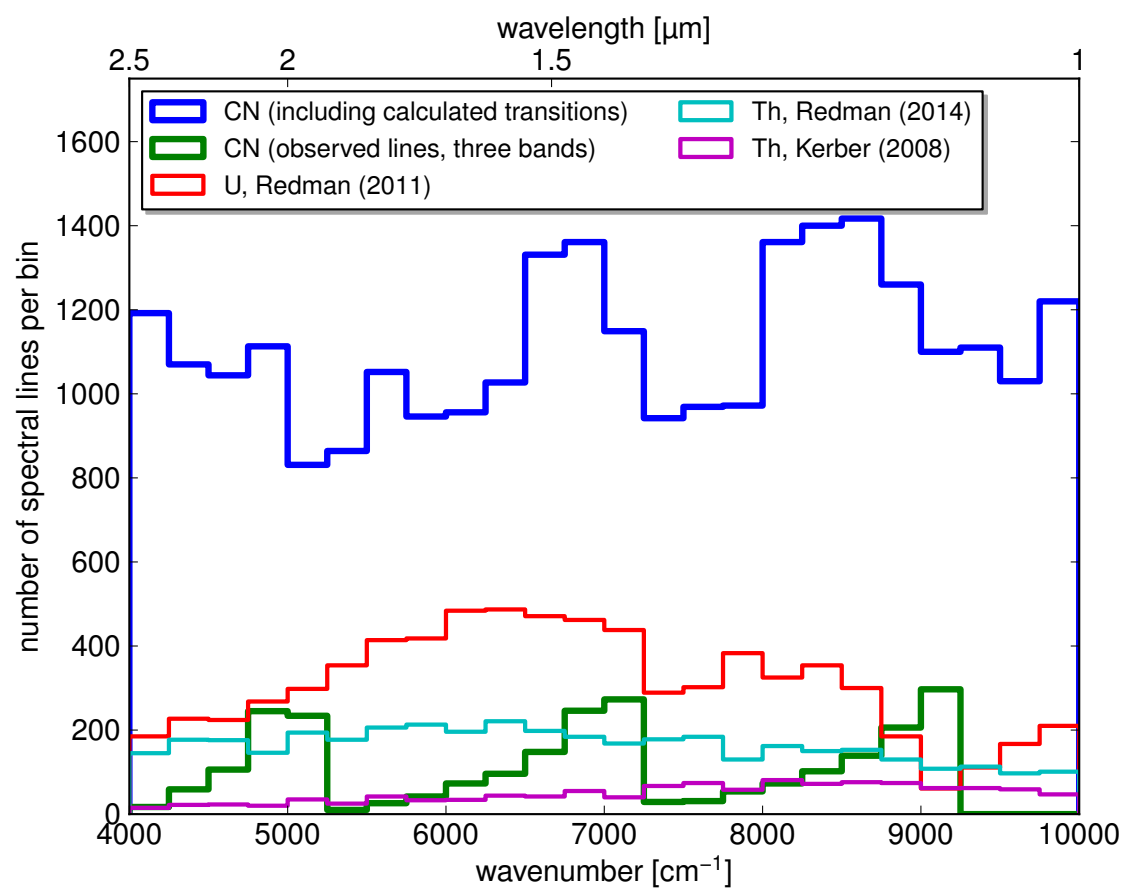

Figure 1.2: Number of documented emission lines for the $C N$ molecule and for species used in $H C L s$ (uranium and thorium) in the near-infrared. The bin width is $250 \mathrm{~cm}^{-1}$. The line list by Brooke et al. (2014) is used for the CN molecule: the green histogram includes only observed lines from the three vibrational bands 0-0,0-1 and 0-2 from the Red System, while the blue histogram includes all calculated transitions from the Red System. Histograms for uranium (U) and thorium (Th) are created with data from Redman et al. (2011), Redman et al. (2014) and Kerber et al. (2008) (red, cyan, and purple curve, respectively).

mixture is nitrogen, and the discharge spectrum also contains many spectral lines from vibrational bands of molecular nitrogen. While analyzing the spectra, it became clear that the many lines of molecular nitrogen in the near-infrared could also be beneficial for wavelength calibration, either in the form of a pure nitrogen spectrum or in combination with emission lines from the $\mathrm{CN}$ molecule.

In addition to a high line density, the molecular emission spectra have more potential advantages in the context of wavelength calibration (compare also Boesch et al., 2014, Boesch \& Reiners, 2015). The frequencies of all lines are defined by physical laws not supposed to change over time, in contrast to Fabry-Perot etalons, and the equipment is relatively inexpensive, in contrast to laser frequency combs. In comparison with HCLs, there will be no contamination from an additional filling gas that produces many strong atomic lines. A low microwave power, low gas temperature and low gas pressure lead to narrow emission lines that are desired in precision spectroscopy. Last but not least, an electrodeless MIP lamp can be expected to be less affected by aging than HCLs because no sputtering processes are involved. There are also aspects of discharge spectra which will pose challenges to the wavelength calibration procedure: as with atomic emission lines from HCLs, the molecular lines are not equally separated and many lines will be blended. 
In this thesis, I attempt to answer the question whether molecular emission spectra, in particular of molecular nitrogen and CN, recorded from a microwave-induced plasma can be used as a wavelength calibration source for near-infrared astrophysical spectrographs. In this context, I analyze the line density, relative line intensities, wavelength stability and aging behavior of molecular spectra that are recorded with a high-resolution Fourier transform spectrometer. The work includes the construction of an experimental setup for an MIP lamp that can potentially be used at a telescope site in the future, i.e., is compact, transportable, and has only little requirements for supply infrastructure.

Application of the MIP lamp in an astrophysical context should not be limited to the potential task of wavelength calibration. The lamp can be used to study spectra from different gaseous species that are of astrophysical interest. For example, the CN molecule has been observed in spectra of different astronomical objects, such as stellar atmospheres (Lambert et al., 1984), the interstellar medium (Meyer \& Jura, 1985) and comets (Fray et al., 2005). I show in Fig. 1.3 a part of the spectrum of the star Arcturus with absorption lines from CN (blue curve on top). The spectrum was published by Hinkle et al. (1995) and they identified a total of 1652 absorption lines from CN. For comparison, I show a discharge spectrum with lines from CN at the bottom of Fig. 1.3 (green curve). The labels are assigned using the line list by Brooke et al. (2014). It can be clearly seen that more $\mathrm{CN}$ lines can be resolved in the lower spectrum because the lines have a smaller width, but all CN transitions that are assigned in the stellar spectrum are also present in the discharge spectrum.

A specific problem in astrophysics that requires laboratory measurements of molecular spectra is the analysis of magnetic fields in cool stars using the Zeeman effect. As an outlook for future work, I will motivate in this thesis the usage of the MIP lamp to study molecular spectra under the influence of a magnetic field and present first experimental steps taken towards the realization of such measurements.

\subsection{Outline of the thesis}

A short overview of the structure of this thesis follows. In Chap. 2, I introduce the radial velocity technique. This method is a prominent example of high-resolution, high-precision spectroscopy and will serve as a physical motivation for the need of stable wavelength calibration. I present a modified version of the algorithm by Anglada-Escudé \& Butler (2012), which allows to disentangle wavelength shifts from spectra of the MIP lamp and of an absorption gas cell serving as a simultaneous relative wavelength reference. The Fourier transform spectrometer that has been used for recording spectra is described in Chap. 3, together with some theoretical background and aspects of data analysis. In Chap. 4, I present the experimental setup with the MIP lamp. The analysis of the discharge spectra is then reported in Chap. 5 with a focus on their usability as wavelength references. In Chap. 6, I discuss an alternative application of the MIP lamp: the investigation of molecular species under the influence of a magnetic field. The modifications to the experimental setup and first calibration measurement are presented. I complete this thesis in Chap. 7 with a summary and an outlook for future work. 


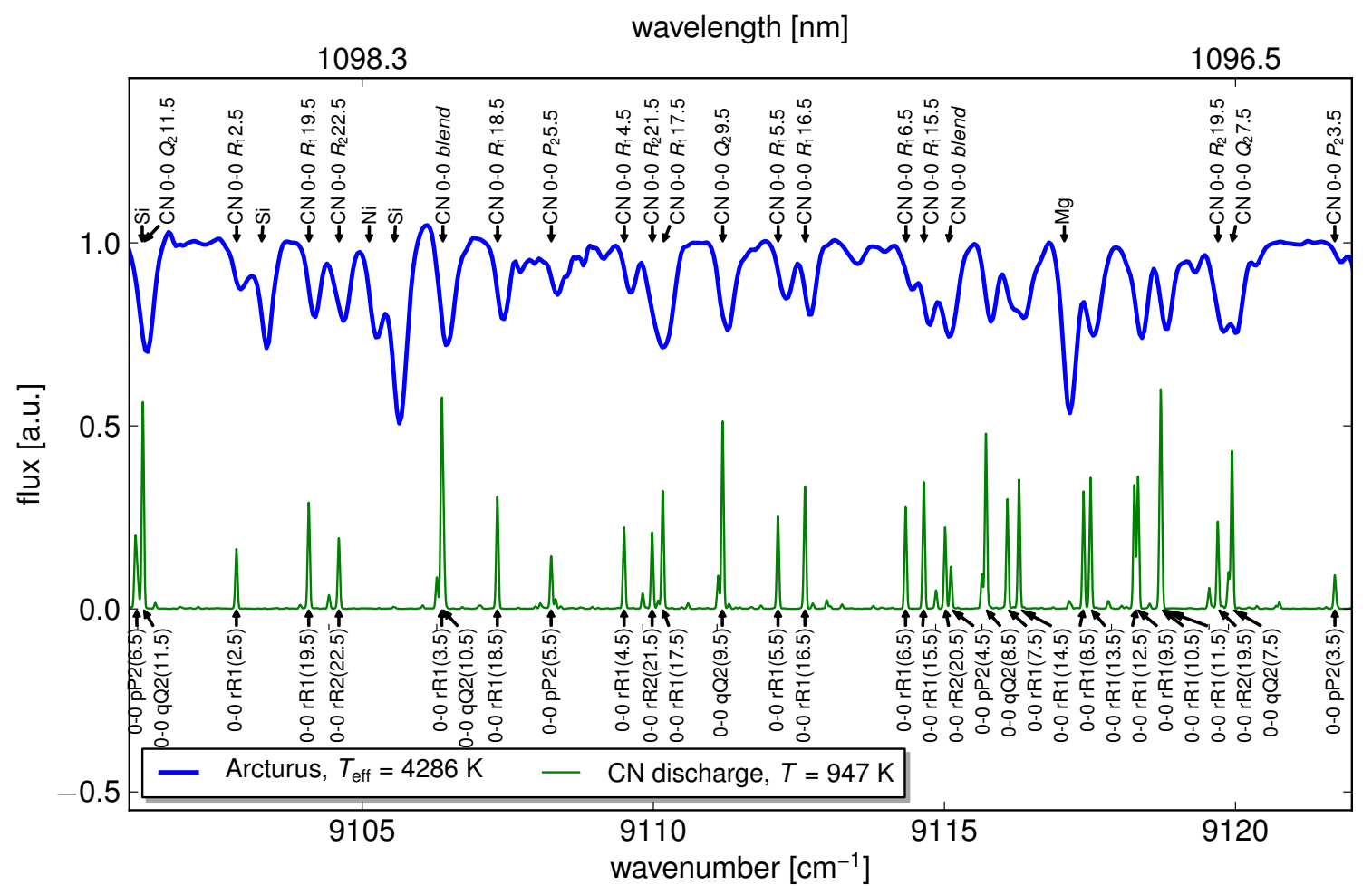

Figure 1.3: On top in blue: Spectrum of the star Arcturus with line identifications from Hinkle et al. (1995). Note the many lines from CN. At the bottom in green: Spectrum of an MIP with emission lines from CN. The transitions are identified using the line list by Brooke et al. (2014). The effective temperature $T_{\text {eff }}$ of Arcturus is taken from Ramirez $\mathcal{G}$ Allende Prieto (2011) and the temperature $T$ for the discharge spectrum is determined from the (temperature broadened) line widths.

Some material of this thesis has been previously published and is reproduced with permission by the Editor in Chief of the journal Astronomy \& Astrophysics (A\&A). Credit: A. Boesch and A. Reiners, A\&A, 582, A43 (2015), reproduced with permission (C) ESO. Some material is submitted to A\&A for publication in the article "Near-infrared wavelength calibration with molecular discharge spectra of nitrogen and CN" (A. Boesch and A. Reiners). The material is reprinted here with agreement of the co-author and I state that I have written the corresponding texts. References to figures, tables and sections have been adjusted to match the structure of this document. In this thesis, commercial products are identified to specify the experimental procedure adequately. The identification is not intended to imply recommendation or endorsement, nor is it intended to imply that the products identified are necessarily the best available for the purpose. 


\section{The radial velocity technique}

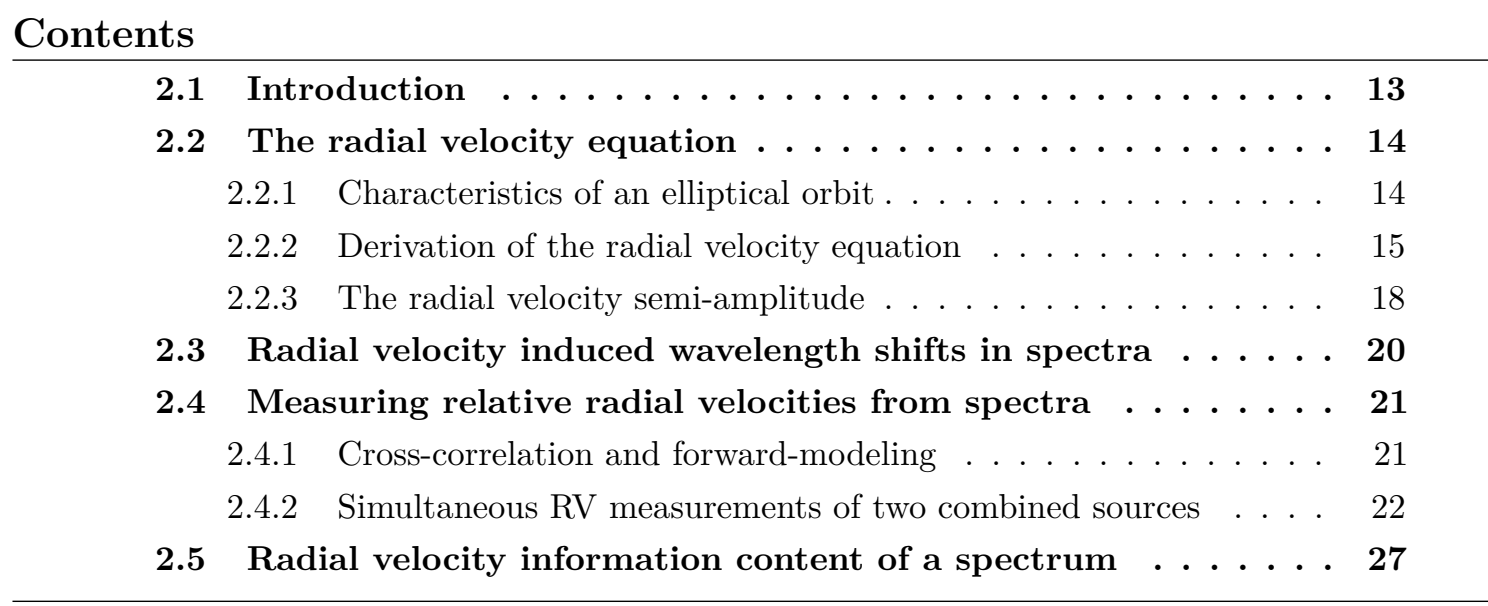

\subsection{Introduction}

The radial velocity (RV) technique is a prominent example for high-precision spectroscopy in astrophysics. It is the most successful method to detect and also confirm extrasolar planets (see Sect. 1.1). This technique allows to determine the velocity of a star along the line of sight to an observer by measuring the wavelength shift of stellar absorption lines in a recorded spectrum. This displacement in wavelength is also called Doppler shift. A periodic variation in the Doppler shift over time can be an indicator for a star's orbital movement around the common barycenter with its planetary companion(s). The concept is illustrated Fig. 2.1. The measurement of radial velocities is not limited to exoplanets' host stars, but has also been applied, e.g., to binary stars and the Sun (e.g., Lu \& Rucinski, 1999, Takeda et al., 2015).

The quest to detect Earth-mass extrasolar planets with the RV method is a major driver in the development of new calibration sources for precision spectroscopy. As astronomers seek to find planets with lower masses that imprint ever smaller signals in the spectroscopic data, instruments need to meet higher demands on stability. When developing calibration sources that establish wavelength solutions for spectrographs, it is therefore common to state the stability of reference lines in velocity units or to say that a calibration technique is stable enough to achieve a certain RV precision, e.g., $1 \mathrm{~m} / \mathrm{s}$.

Reliable wavelength calibration sources are needed for many spectroscopic measurements. However, the requirements on wavelength stability and wavelength coverage are arguably 


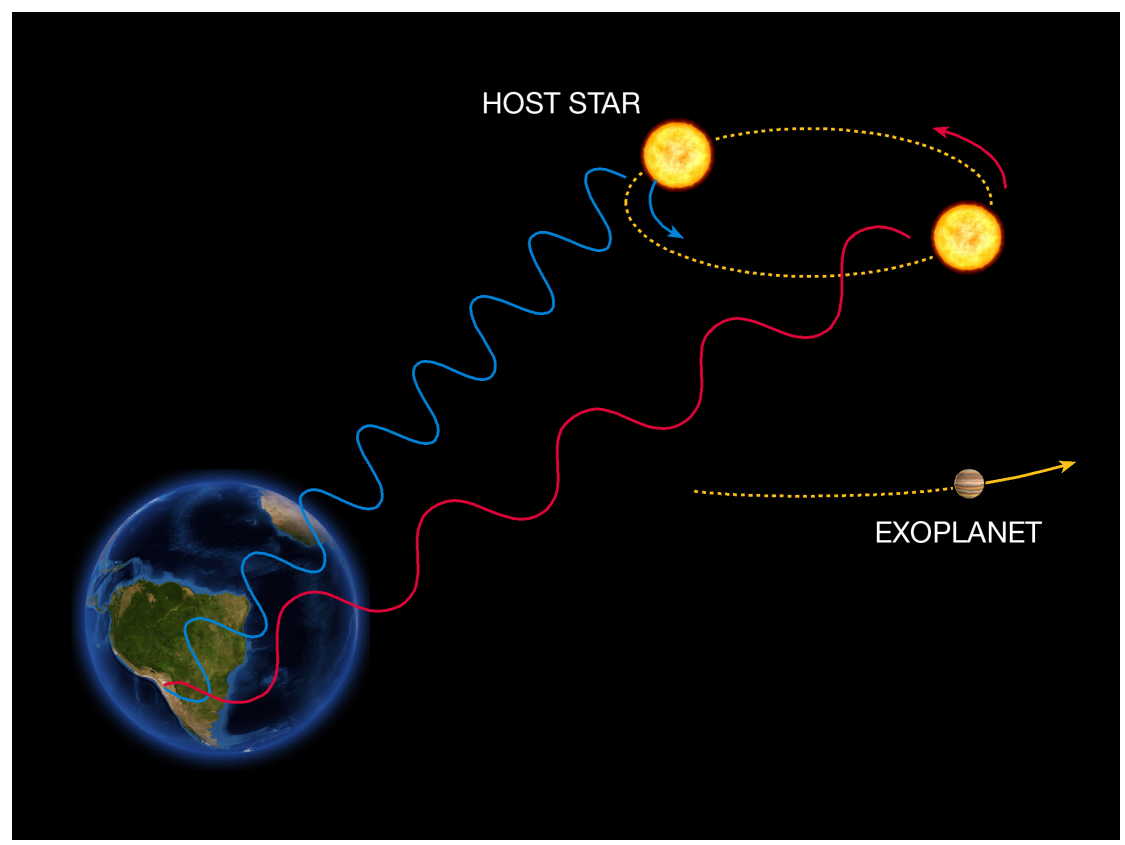

Figure 2.1: Artist's impression of the radial velocity method. The host star orbits around the common center of mass of the star-planet system. When the star moves towards the observer on Earth, its spectrum is blue-shifted (indicated by the blue wave). It is red-shifted when the star moves away. Image from ESO (www.eso.org/public/images/eso0722e).

among the highest for current and future precision RV observations. Therefore, I introduce the RV technique in detail in this section. In Sect. 2.2, I derive the equation which links a measured RV to the physical properties of a star's orbit. The determination of the RV from the Doppler shift in the spectrum is discussed in Sect. 2.3. In Sect. 2.4 follows a description of the algorithm that is used to determine the RV stability of laboratory spectra recorded with a Fourier transform spectrometer (FTS). Finally, the precision limit for RV measurements is discussed in Sect. 2.5.

\subsection{The radial velocity equation}

The objective of the following derivation is to find a mathematical expression for the radial velocity $v_{\text {rad }}$, i.e., the velocity along the line of sight to the observer on Earth, of a star due to gravitational interaction with an orbiting body. Detailed descriptions of the RV method can be found in several textbooks, e.g., by Seager (2010) or Perryman (2011). Here, I present a concise, yet complete derivation that leads to Eq. 2.15.

\subsubsection{Characteristics of an elliptical orbit}

The mutual motion of two gravitationally bound objects, such as a planet and its host star, is governed by Newton's Law of Gravitation and Laws of motion. In preparation for the 
following derivation of the RV equation, I introduce characteristic quantities of an elliptical orbit and some fundamental relations. The bodies move on elliptical orbits around a common center of mass. Strictly speaking, three different orbits can be identified: the orbit of the star around the common barycenter, the orbit of the planet around the barycenter and the relative orbit of the two bodies. The last one can also be understood as the planet orbiting around the star if the coordinate system is chosen accordingly. Following Hilditch (2001, Sect. 2.3) and Perryman (2011, Sect. 2.1), I consider the three parameters orbital period $P$, semi-major axis $a$, and orbital eccentricity $e$. The quantities for the barycentric orbit of the star are labeled with subscript "s", those of the planet with subscript "p" and the relative orbit with "rel". It follows directly from the geometry of the problem that

$$
e_{\mathrm{s}}=e_{\mathrm{p}}=e_{\mathrm{rel}} \equiv e \quad \text { and } \quad P_{\mathrm{s}}=P_{\mathrm{s}}=P_{\text {rel }} \equiv P
$$

Kepler's Third Law can be written in the following ways when it is applied to the three orbits:

for the relative orbit:

for the barycentric orbit of the star:

for the barycentric orbit of the planet:

$$
\begin{aligned}
\frac{4 \pi^{2} a_{\mathrm{rel}}^{3}}{P^{2}} & =G\left(M_{\mathrm{s}}+M_{\mathrm{p}}\right) \\
\frac{4 \pi^{2} a_{\mathrm{s}}^{3}}{P^{2}} & =\frac{G M_{\mathrm{p}}^{3}}{\left(M_{\mathrm{s}}+M_{\mathrm{p}}\right)^{2}} \\
\frac{4 \pi^{2} a_{\mathrm{p}}^{3}}{P^{2}} & =\frac{G M_{\mathrm{s}}^{3}}{\left(M_{\mathrm{s}}+M_{\mathrm{p}}\right)^{2}}
\end{aligned}
$$

In addition to the orbital parameters, the masses of the star and the planet, $M_{\mathrm{S}}$ and $M_{\mathrm{p}}$, and the gravitational constant $G$ have been introduced. From Eqs. 2.1 to 2.3 we can infer that the semi-major axes and masses for the three orbits are in the following proportions

$$
a_{\mathrm{s}}: a_{\mathrm{p}}: a_{\mathrm{rel}}=M_{\mathrm{p}}: M_{\mathrm{s}}:\left(M_{\mathrm{s}}+M_{\mathrm{p}}\right)
$$

In the remainder of the text, the subscripts "s", "p" and "rel" are suppressed if the notation is clear from the context.

An elliptical, or Keplerian, orbit is fully described by seven parameters (e.g., Perryman, 2011, Sect. 2.1). I already introduced the orbital period $P$, semi-major axis $a$, and orbital eccentricity $e$. In addition, the orientation of the orbital plane on the sky and the timedependent position of the orbiting body are described by four angles: the longitude of the ascending node $\Omega$, the inclination $i$, the argument of periapsis $\omega$ and the true anomaly $\nu$. Figure 2.2 shows an elliptical orbit which I simulated and illustrated with the following parameters: $e=0.5, \Omega=325^{\circ}, i=30^{\circ}, \omega=70^{\circ}$ and $\nu=72^{\circ}$.

\subsubsection{Derivation of the radial velocity equation}

The quantity $z$ (marked in red color in Fig. 2.2) is the distance between the star and the barycenter projected onto the line of sight that is perpendicular to the plane of sky. We obtain the radial velocity if we calculate the change in this distance over time:

$$
v_{\text {rad }}=\frac{\mathrm{d} z}{\mathrm{~d} t} .
$$




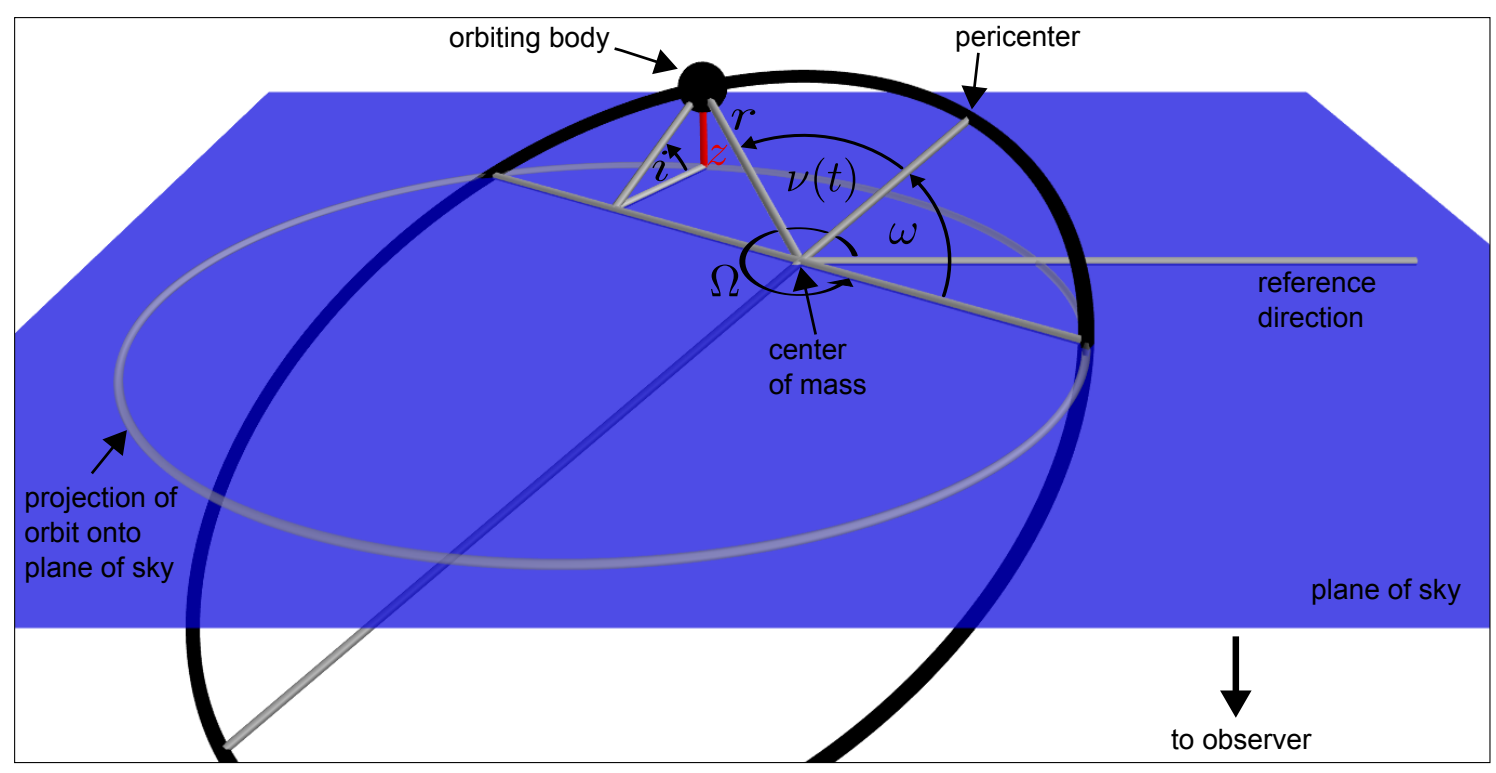

Figure 2.2: Orbital parameters of an elliptical orbit. The black dot designated as "orbiting body" is the host star orbiting around the barycenter of the star-planet system. The planet itself is not shown.

We can express the distance $z$ in terms of the three angles $i, \nu, \omega$ and the length of the position vector $r$ using trigonometric relations in two right-angle triangles (see Fig. 2.2). From the triangle containing the inclination $i$ as one angle, we obtain the relation

$$
z=\tilde{z} \cdot \sin (i)
$$

and using the triangle in the orbital plane, we can write

$$
\tilde{z}=r \cdot \sin (\pi-(\nu+\omega))=r \cdot \sin (\nu+\omega) .
$$

The last step is valid without loss of generality concerning the position of the orbiting body on the ellipse, because of the periodic properties of the sine function. Combining Eqs. 2.6 and 2.7, we can substitute $z$ in Eq. 2.5:

$$
v_{\mathrm{rad}}=\frac{\mathrm{d}}{\mathrm{d} t}[r \cdot \sin (\nu+\omega) \cdot \sin (i)]
$$

The radius $r$ and the true anomaly $\nu$ are time dependent, i.e., $r=r(t)$ and $\nu=\nu(t)$, while it is assumed that $\omega$ is constant over time (no apsidal precession). We carry out the differentiation with respect to time and obtain:

$$
\begin{aligned}
& v_{\mathrm{rad}}=r \cdot \frac{\mathrm{d}}{\mathrm{d} t} \sin (\nu+\omega) \cdot \sin (i)+\frac{\mathrm{d} r}{\mathrm{~d} t} \cdot \sin (\nu+\omega) \cdot \sin (i) \\
& v_{\mathrm{rad}}=[r \dot{\nu} \cos (\nu+\omega)+\dot{r} \sin (\nu+\omega)] \cdot \sin (i) .
\end{aligned}
$$

In the next steps, we find expressions for $r \dot{\nu}$ and $\dot{r}$ (Eqs. 2.12 and 2.13) in order to eliminate these quantities from Eq. 2.8. The radius $r$ of an ellipse can be written as (e.g., Perryman, 
2011, Sect. 2.1):

$$
r=\frac{\left(1-e^{2}\right) a}{1+e \cos (\nu)},
$$

where $a$ is the semi-major axis and $e=\sqrt{1-b^{2} / a^{2}}$ is the eccentricity of the ellipse ( $b$ is the semi-minor axis). We take the time derivative to find an expression for $\dot{r}$. Note that $\nu=\nu(t)$ is time-dependent, while $e$ and $a$ are constant quantities:

$$
\begin{aligned}
& \dot{r}=\frac{[1+e \cos (\nu)] \cdot \frac{\mathrm{d}}{\mathrm{d} t}\left[\left(1-e^{2}\right) a\right]-\left(1-e^{2}\right) a \cdot \frac{\mathrm{d}}{\mathrm{d} t}[1+e \cos (\nu)]}{[1+e \cos (\nu)]^{2}} \\
& \dot{r}=\frac{\left(1-e^{2}\right) a}{1+e \cos (\nu)} \cdot \frac{\dot{\nu} e \sin (\nu)}{1+e \cos (\nu)} \\
& \dot{r} \stackrel{\text { Eq. }}{=} 2.9 r \dot{\nu} \cdot \frac{e \sin (\nu)}{1+e \cos (\nu)} .
\end{aligned}
$$

We use Kepler's Second Law to find an expression for $r \dot{\nu}$. The Law states that a line drawn from the orbiting body to the focus of the ellipse sweeps out equal areas in equal times. In a time step $\mathrm{d} t$, this line covers a small triangle with side lengths of $r$ and $r \mathrm{~d} \nu$ resulting in an area of

$$
\mathrm{d} A=\frac{1}{2} \cdot r \cdot r \mathrm{~d} \nu,
$$

and an areal velocity of

$$
\frac{\mathrm{d} A}{\mathrm{~d} t}=\frac{1}{2} r^{2} \dot{\nu} .
$$

This equation can be solved for $A$ by separation of variables and subsequent integration:

$$
\begin{aligned}
\int \mathrm{d} A & =\int \frac{1}{2} r^{2} \dot{\nu} \mathrm{d} t \\
A & =\frac{1}{2} r^{2} \dot{\nu} t+C .
\end{aligned}
$$

After one orbital period $P$, the star has traveled around the whole ellipse and $A(t=$ $P)=A_{\text {ellipse }}$. We find that the integration constant is $C=0$ and using $A_{\text {ellipse }}=\pi a b=$ $\pi a \sqrt{a^{2}\left(1-e^{2}\right)}$, we obtain:

$$
r \dot{\nu}=\frac{1}{r} \cdot \frac{2 \pi a^{2} \sqrt{1-e^{2}}}{P} .
$$

In the final step to find the expressions to eliminate $r \dot{\nu}$ and $\dot{r}$ from the RV equation, we need to correct for the term $r$ in Eq. 2.11 and for the term $r \dot{\nu}$ in Eq. 2.10. Therefore, we substitute the expression for $r$ (Eq. 2.9) into Eq. 2.11, and then plug the result for $r \dot{\nu}$ into Eq. 2.10. The results are the two targeted equations:

$$
\begin{aligned}
r \dot{\nu} & =\frac{1+e \cos (\nu)}{\left(1-e^{2}\right) a} \cdot \frac{2 \pi a^{2} \sqrt{1-e^{2}}}{P}=\frac{2 \pi a[1+e \cos (\nu)]}{P \sqrt{1-e^{2}}} \text { and } \\
\dot{r} & =\frac{2 \pi a[1+e \cos (\nu)]}{P \sqrt{1-e^{2}}} \cdot \frac{e \sin (\nu)}{1+e \cos (\nu)}=\frac{2 \pi a e \sin (\nu)}{P \sqrt{1-e^{2}}} .
\end{aligned}
$$


Equations 2.12 and 2.13 can be substituted into the formula for the radial velocity (Eq. 2.8) to obtain an expression that only depends on orbital parameters:

$$
\begin{aligned}
& v_{\mathrm{rad}}=\left[\frac{2 \pi a(1+e \cos (\nu))}{P \sqrt{1-e^{2}}} \cdot \cos (\nu+\omega)+\frac{2 \pi a e \sin (\nu)}{P \sqrt{1-e^{2}}} \cdot \sin (\nu+\omega)\right] \cdot \sin (i) \\
& v_{\mathrm{rad}}=\underbrace{\frac{2 \pi a \sin (i)}{P \sqrt{1-e^{2}}} \cdot[\cos (\nu+\omega)+e \cos (\nu) \cos (\nu+\omega)+e \sin (\nu) \sin (\nu+\omega)] .}_{K} .
\end{aligned}
$$

Here I introduce the quantity $K$ which includes all time-independent factors. It is called the radial velocity semi-amplitude (e.g., Perryman, 2011, Sect. 2.1) and will be discussed in the next section. In order to simplify the term in the squared brackets, the following trigonometric identities may be used (e.g., Woan, 2000):

$$
\begin{aligned}
\cos (x+y) & =\cos (x) \cos (y)-\sin (x) \sin (y) \quad, \\
\sin (x+y) & =\sin (x) \cos (y)+\cos (x) \sin (y) \quad \text { and } \\
1 & =\sin ^{2}(x)+\cos ^{2}(x) .
\end{aligned}
$$

Using these equations, Eq. 2.14 can be simplified and we obtain the final RV equation:

$$
v_{\text {rad }}=K[\cos (\nu+\omega)+e \cos (\omega)]
$$

The radial velocity $v_{\text {rad }}$ is a function of the true anomaly $\nu$ which changes with time. The shape of the RV curve depends on the eccentricity $e$ and the argument of periapsis $\omega$. For a circular orbit $(e=0)$, the RV signal follows an undisturbed cosine curve. The amplitude of the RV signal depends on the factor $K$. Figure 2.3 shows radial velocity measurements of the star $\iota$ Horologii over several years. A clear RV curve with a semi-amplitude of about $70 \mathrm{~m} / \mathrm{s}$ becomes visible when the data points from observations taken at different times are folded with the estimated orbital period.

\subsubsection{The radial velocity semi-amplitude}

In the previous section, we derived the radial velocity equation (Eq. 2.15) and introduced the host star's radial velocity semi-amplitude:

$$
K=\frac{2 \pi a_{s} \sin (i)}{P \sqrt{1-e^{2}}} .
$$

In this section, an expression for $K$ is derived that depends only on the mass of the star $M_{\mathrm{s}}$, the mass of the planet $M_{\mathrm{p}}$ and the distance between the two of them $a_{\mathrm{rel}}$. This allows to estimate the maximum radial velocity from the physical parameters of the star-planet system. 

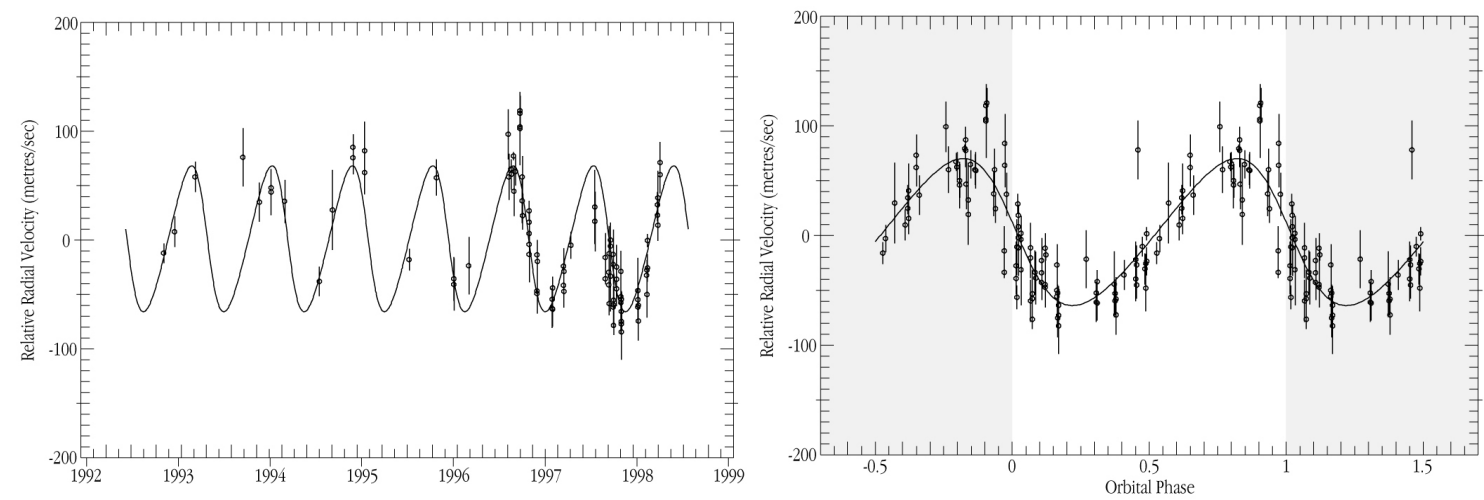

Figure 2.3: Radial velocity variations of ८ Horologii over a period of nearly six years (left panel) and folded onto the orbital period of 320 days (right panel). The curved line indicates the variation that is caused by the orbiting planet determined from a best-fit Keplerian orbit. The planet approaches the observer between orbital phase 0.5 and 1 , when the star receeds and the radial velocity is positive. The planet is in front of the star at phase 0 when the radial velocity is zero and behind the star at phase 0.5. Graphs are from ESO (www.eso.org/public/images/eso9938a and www.eso.org/public/images/eso9938b).

From from Eqs. 2.1 and 2.4 we already know $P^{2}=4 \pi^{2} a_{\text {rel }}^{3} /\left(G\left(M_{\mathrm{s}}+M_{\mathrm{p}}\right)\right)$ and $a_{\mathrm{s}}=$ $M_{\mathrm{p}} a_{\mathrm{rel}} /\left(M_{\mathrm{s}}+M_{\mathrm{p}}\right)$. Thus, we can write:

$$
\begin{aligned}
K & =2 \pi \cdot a_{s} \cdot \frac{1}{P} \cdot \frac{\sin (i)}{\sqrt{1-e^{2}}} \\
& =2 \pi \cdot \frac{M_{\mathrm{p}} a_{\mathrm{rel}}}{M_{\mathrm{s}}+M_{\mathrm{p}}} \cdot \frac{G^{\frac{1}{2}}\left(M_{\mathrm{s}}+M_{\mathrm{p}}\right)^{\frac{1}{2}}}{2 \pi a_{\mathrm{rel}}^{\frac{3}{2}}} \cdot \frac{\sin (i)}{\sqrt{1-e^{2}}} \\
& =G^{\frac{1}{2}} \cdot a_{\mathrm{rel}}^{-\frac{1}{2}} \cdot M_{\mathrm{p}} \cdot\left(M_{\mathrm{s}}+M_{\mathrm{p}}\right)^{-\frac{1}{2}} \cdot \frac{\sin (i)}{\sqrt{1-e^{2}}} .
\end{aligned}
$$

It can be assumed that the mass of the planet is much smaller than the mass of the star, i.e., $M_{\mathrm{s}}+M_{\mathrm{p}} \approx M_{\mathrm{s}}$. To express the formula in quantities from the Solar System, we give the mass of the planet $M_{\mathrm{p}}$ in Earth masses $\left(\mathrm{M}_{\oplus}=5.974 \cdot 10^{24} \mathrm{~kg}\right)$, the mass of the star $M_{s}$ in solar masses $\left(\mathrm{M}_{\odot}=1.989 \cdot 10^{30} \mathrm{~kg}\right)$ and the orbiting distance $a_{\text {rel }}$ in astronomical units $\left(\mathrm{AU}=1.496 \cdot 10^{11} \mathrm{~m}\right)$. The gravitational constant has the value $G=6.67398 \mathrm{~m}^{3} \mathrm{~kg} \mathrm{~s}^{-2}$. The numerical values are taken from Karttunen et al. (2003) and the constant term becomes

$$
G^{\frac{1}{2}} \cdot(1 \mathrm{AU})^{-\frac{1}{2}} \cdot\left(1 \mathrm{M}_{\oplus}\right) \cdot\left(1 \mathrm{M}_{\odot}\right)^{-\frac{1}{2}}=0.0895 \frac{\mathrm{m}}{\mathrm{s}},
$$

This leads to a descriptive expression for the radial velocity semi-amplitude:

$$
K=0.0895 \cdot\left(\frac{a_{\mathrm{rel}}}{1 \mathrm{AU}}\right)^{-\frac{1}{2}}\left(\frac{M_{\mathrm{p}}}{1 \mathrm{M}_{\oplus}}\right)\left(\frac{M_{\mathrm{s}}}{1 \mathrm{M}_{\odot}}\right)^{-\frac{1}{2}} \frac{\sin (i)}{\sqrt{1-e^{2}}} \frac{\mathrm{m}}{\mathrm{s}}
$$


It can be seen directly from Eq. 2.16 that an Earth-mass planet orbiting a star with the mass of the Sun in a circular orbit at a distance of 1 AU creates a RV signal with an amplitude of $8.95 \mathrm{~cm} / \mathrm{s}$. This is less than a tenth of the preferred walking speed of humans which tends to be $1.4 \mathrm{~m} / \mathrm{s}$ (Browning et al., 2006). For comparison, the Doppler shift introduced by Jupiter on the Sun is about $12.5 \mathrm{~m} / \mathrm{s}$. Keeping all the other parameters constant, the RV amplitude increases for higher-mass planets, lower-mass host stars or smaller separations. From this point of view, it is not surprising that many of the first discovered exoplanets using the RV method are so called "Hot Jupiters"; planets with masses similar to Jupiter's that orbit close to their star. An example for a Hot Jupiter is the first exoplanet detected around a main-sequence star (51 Pegasi b; Mayor \& Queloz, 1995).

\subsection{Radial velocity induced wavelength shifts in spectra}

The radial velocity of a star is determined by measuring the simultaneous wavelength shift of the many absorption lines that make up the star's spectrum (e.g., Perryman, 2011, Sect. 2.2). When radiation of wavelength $\lambda_{\text {em }}$ is emitted from an object traveling at a velocity $\hat{v}$ with respect to the observer, the observed wavelength $\lambda_{\text {obs }}$ is given by the relativistic Doppler effect (e.g., Kang, 1999):

$$
\lambda_{\mathrm{obs}}=\lambda_{\mathrm{em}} \cdot \frac{1+\frac{\hat{v}}{c} \cos (\Theta)}{\left(1-\left(\frac{\hat{v}}{c}\right)^{2}\right)^{1 / 2}},
$$

where $\hat{v}>0$ if the star recedes from the observer and $\hat{v}<0$ if the star approaches the observer. I use the hat-notation, $\hat{v}$, to distinguish the velocity from the radial velocity $v_{\text {rad }} \equiv v=\hat{v} \cos (\Theta)$. The angle $\Theta$ is located between the velocity vector and the radiation wave vector towards the observer. The speed of light is given by $c$. The relativistic correction can be neglect for $\hat{v} \ll c$ :

$$
\lambda_{\mathrm{obs}}=\lambda_{\mathrm{em}} \cdot\left(1+\frac{\hat{v}}{c} \cos (\Theta)\right)=\lambda_{\mathrm{em}} \cdot\left(1+\frac{v}{c}\right) .
$$

The shift in wavelength $\Delta \lambda$ between the emitted and observed light can now be written as:

$$
\Delta \lambda=\lambda_{\mathrm{obs}}-\lambda_{\mathrm{em}}=\lambda_{\mathrm{em}} \cdot \frac{v}{c} .
$$

This leads to an expression for the radial velocity $v_{\text {rad }} \equiv v$ from a measurement of the wavelength difference in a spectrum:

$$
v=\frac{\Delta \lambda}{\lambda_{\mathrm{em}}} \cdot c
$$

The effect of a Doppler shift on a spectral line is illustrated in Fig. 2.4. A Gaussian profile represents a single absorption line. The full width at half maximum (FWHM) is 
set to $0.01 \mathrm{~nm}$ and the depth is normalized to the range $[0,1]$. The unshifted line (blue) is centered at $630.25 \mathrm{~nm}$. This is the position of an iron absorption line in the solar spectrum which is shown for comparison (yellow dashed curve). A narrow telluric oxygen line is also present in the observed data next to the broader Fe line. In the left panel, the red curve is shifted by $5 \mathrm{~km} / \mathrm{s}$, with respect to the blue curve. This shift corresponds approximately to the FWHM of the Fe line. In the right panel, the red curve is shifted by $500 \mathrm{~m} / \mathrm{s}$ which corresponds to a wavelength difference of approximately $10^{-3} \mathrm{~nm}$. As already mentioned above (see discussion after Eq. 2.16), an Earth-mass planet in an orbit around a Sun-mass star at a distance of $1 \mathrm{AU}$ would induce a RV shift of only about $10 \mathrm{~cm} / \mathrm{s}$. This is 5000 times less than the shift shown in the right panel of Fig. 2.4. The corresponding wavelength shift is of the order of $10^{-7} \mathrm{~nm}$. In return, Eqs. 2.16 and 2.18 can be used to calculate the wavelength stability that a reference source needs to provide to detect an exoplanet from RV measurements taken at different times. In practice, the information from one spectral line is not sufficient for precise RV measurements with Doppler shifts at the $\mathrm{m} / \mathrm{s}$ level. Instead, astronomers use spectra with many lines to reduce the uncertainty in the RV measurement (see also Sect. 2.5). Different methods have been developed to extract the RV from spectra and I discuss some of them in the next section.
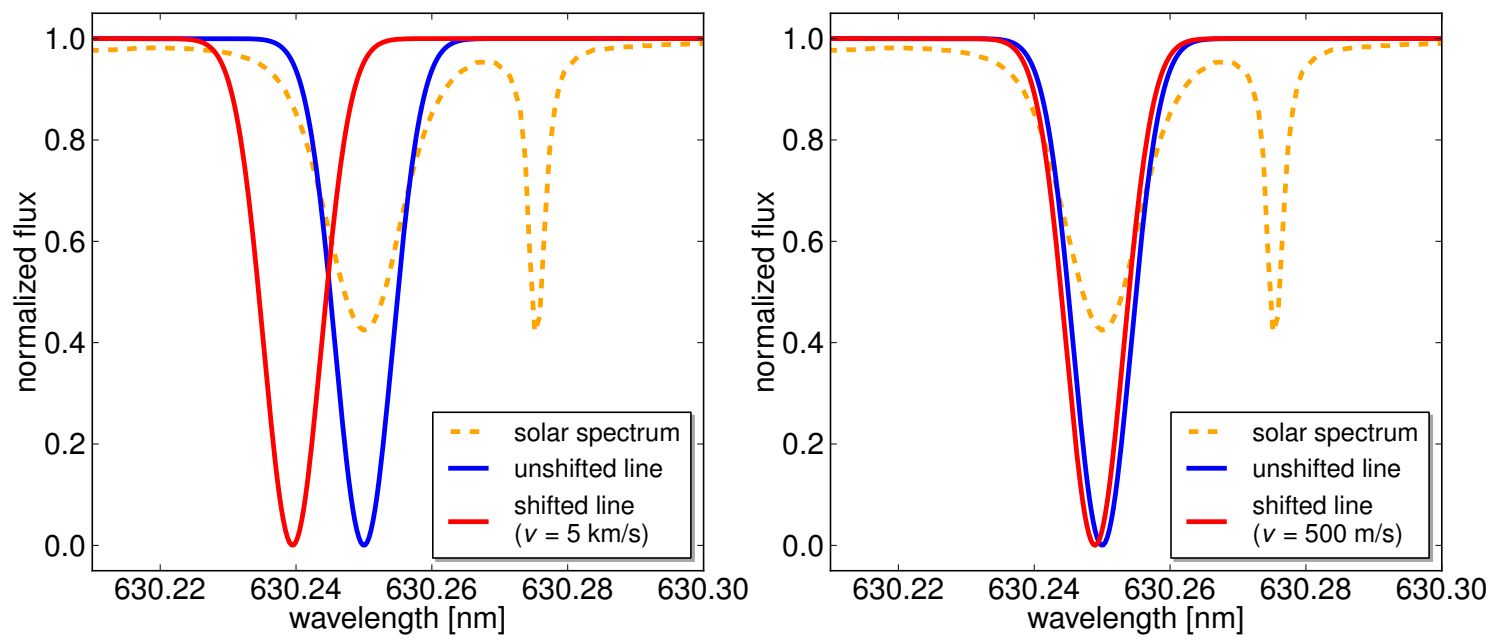

Figure 2.4: Illustration of spectral line shifts due to different radial velocities. The red curve is shifted with respect to the blue curve. The solar spectrum recorded by Kurucz et al. (1984) is shown for comparison (yellow curve).

\subsection{Measuring relative radial velocities from spectra}

\subsubsection{Cross-correlation and forward-modeling}

A crucial task in radial velocity measurements is the extraction of the Doppler shift from the observed spectrum. Two techniques are widely used in the exoplanet community: cross-correlation and forward-modeling (Lovis \& Fischer, 2010). Further methods include 
fitting of line profiles or the mirroring method (Parimucha \& Škoda, 2007). Radial velocity measurements usually consist of many observations of the same source taken at different times (a time series of observations). Most RV measurements do not need absolute wavelength solutions, but are relative with respect to another spectrum. A template or master spectrum is needed to serve as the reference to which all other observations are compared. The template can be recorded in a separate observation, or created by averaging the observations from the time series. It should have a high signal-to-noise ratio.

An efficient way to compute the global shift between two similar spectra is cross-correlation (Lovis \& Fischer, 2010). A spectrum is cross-correlated with the template spectrum and the cross-correlation function has its maximum at the RV offset between the two spectra. In practice, it takes a lot of time and effort to record a high quality template for each source, e.g., each star. Instead of using an individual template for each star, the cross-correlation can be carried out with a weighted binary mask. This mask is nonzero only at the line positions. CORAVEL (CORrelation-RAdial-VELocities) was one of the first spectrographs to be used with this method that has been refined over the years, e.g., for the HARPS spectrograph (Baranne et al., 1979, Queloz, 1995, Pepe et al., 2002, Mayor et al., 2003).

Anglada-Escudé \& Butler (2012) proposed a forward modeling algorithm under the HARPSTERRA project. It is based on minimizing the difference of the observed spectrum against a parameterized template. The individual spectra need to be wavelength calibrated. For HARPS, which is a highly stabilized instrumented, this is achieved using a hollow cathode lamp that is recorded simultaneously with the science exposure using a second calibration fiber. Anglada-Escudé \& Butler (2012) define the difference between the template $F$ and another observed spectrum $f$ at each wavelength $\lambda$ as

$$
R[\lambda, \hat{\alpha}]=F\left[\alpha_{\mathrm{v}} \lambda\right]-f[\lambda] \cdot \sum_{m=0}^{M} \alpha_{m}\left(\lambda-\lambda_{\mathrm{c}}\right)^{m},
$$

with a set of fitting parameters $\hat{\alpha}=\left\{\alpha_{\mathrm{v}}, \alpha_{0}, \ldots, \alpha_{M}\right\}$. The free parameter $\alpha_{\mathrm{v}}$ corresponds to the RV offset between the two spectra. The spectrum $f$ is multiplied by a polynomial to correct for changes in the intensity caused by variability in the blaze function. According to Anglada-Escudé \& Butler (2012), this variability can be related to several instrumental or observational effects, such as atmospheric differential refraction. They use a third order polynomial for the flux correction. In an iterative process, the set of parameters $\hat{\alpha}$ is calculated which minimizes the difference $R$ over all wavelengths in a least-squares fashion. The subtraction of the central wavelength $\lambda_{c}$ helps to avoid numerical problems with large numbers. In the next section, I describe how I modified this algorithm to calculate the wavelength stability of spectra recorded with the FTS.

\subsubsection{Simultaneous RV measurements of two combined sources}

Spectra recorded with an FTS have an intrinsic wavelength scale that is based on the internal HeNe laser (see Chap. 3 for more information on the functionality of an FTS). The spectra are automatically wavelength calibrated and we can use the methods described in the previous section to measure the Doppler shift relative to a template. However, the 
wavelength solution of the FTS cannot be expected to be stable on a $1 \mathrm{~m} / \mathrm{s}$ level due to instrumental and environmental influences. An example for instrumental effects is the frequency stability of the internal laser which is referenced by the manufacturer to be $1.5 \mathrm{~m} / \mathrm{s}$ over 24 hours (stabilized HeNe laser SIOS SL-04). Environmental effects include variations of pressure, temperature or outgassing inside the instrument. It is difficult to predict to which extend these variations influence the wavelength scale because the exact conditions inside the instrument are not known. A strategy to overcome the stability limitations is to simultaneously measure another calibration device which is expected to be stable. The calibration source monitors the wavelength drift of the measuring system and serves as a relative wavelength reference. This device can, e.g., be a gas absorption cell as it is used for wavelength calibration of astrophysical spectrographs. To measure the wavelength stability of the spectra from the microwave-induced plasma (MIP) lamp, I decided to combine its emission spectrum with the absorption spectrum of a $\mathrm{C}_{2} \mathrm{H}_{2}$ absorption cell. Using a beam-splitter, the light from the MIP lamp is combined with light from a halogen lamp illuminating the $\mathrm{C}_{2} \mathrm{H}_{2}$ cell. The experimental configuration is explained later in detail in Sect. 4.3. The resulting spectrum $f_{\text {com }}$ is a superposition of the emission spectrum from the MIP lamp and the absorption spectrum of the $\mathrm{C}_{2} \mathrm{H}_{2}$ cell with a blackbody contribution from the halogen lamp. Figure 5.17 in Sect. 5.3 shows such a spectrum.

I modified the HARPS-TERRA algorithm introduced above to measure and disentangle the individual Doppler shifts of the two sources. The principle of the algorithm is also outlined in Sect. 5.3 with a concrete application to the MIP lamp and a $\mathrm{C}_{2} \mathrm{H}_{2}$ absorption cell. Here, I show the full analytical implementation of the modeling procedure in a general notation for two different light sources with individual template spectra $F_{1}, F_{2}$ and an observed spectrum $f_{\text {com }}$ with simultaneous (combined) illumination. The concept of the method is illustrated in Fig. 2.5. The starting point for the algorithm is Eq. 2.19 which is modified to model the spectral information from two sources:

$$
\begin{aligned}
R[\lambda, \hat{\alpha}] & =F_{1}\left[\alpha_{1, \mathrm{v}} \lambda\right] \cdot \sum_{m=0}^{M} \alpha_{1, m}\left(\lambda-\lambda_{\mathrm{c}}\right)^{m} \\
& +F_{2}\left[\alpha_{2, \mathrm{v}} \lambda\right] \cdot \sum_{l=0}^{L} \alpha_{2, l}\left(\lambda-\lambda_{\mathrm{c}}\right)^{l}-f_{\mathrm{com}}[\lambda] .
\end{aligned}
$$

The two templates $F_{1}, F_{2}$ are separate recordings of the two individual sources. They are parameterized with individual fitting parameters for their Doppler shifts, $\alpha_{1, \mathrm{v}}$ and $\alpha_{2, \mathrm{v}}$. In addition to the wavelength shifts, a polynomial for each template is used to correct for intensity changes. Experience shows that a constant or linear polynomial is sufficient because the illumination is very stable. In contrast to Eq. 2.19 , the polynomial is applied to the template $F$ and not to the spectrum $f_{\text {com }}$ of the time-series. Anglada-Escudé \& Butler (2012) write that this would couple the flux normalization coefficients to the Doppler factor in a nonlinear fashion, which would be an undesirable complication from a numerical point of view. For the new application with two templates, the formula needs to be adjusted with the notation in Eq. 2.20 and no limitations in the execution of the algorithm have been noticed. The variable $\hat{\alpha}$ comprises all fitting parameters for better readability, e.g., 
$\hat{\alpha}=\left\{\alpha_{1, \mathrm{v}}, \alpha_{1,0}, \alpha_{1,1}, \alpha_{2, \mathrm{v}}, \alpha_{2,0}, \alpha_{2,1}\right\}$ for the case with two linear polynomials. This case is considered in the following calculations for better readability. The two templates $F_{1}$, $F_{2}$ as well as the combined spectrum $f_{\text {com }}$ are convolved with the instrumental profile, or point spread function $P S F$, of the FTS. Due to the distributivity of the convolution, $\left(F_{1} * P S F\right)+\left(F_{2} * P S F\right)=\left(F_{1}+F_{2}\right) * P S F$, this does not influence the algorithm as long as the instrumental profile is stable during the observation run. In the remainder of this section, I present the mathematical algorithm to calculate the RV shifts of the two templates, $F_{1}$ and $F_{2}$, to match the spectrum of the two combined light sources. I adopt the notation from Anglada-Escudé \& Butler (2012).
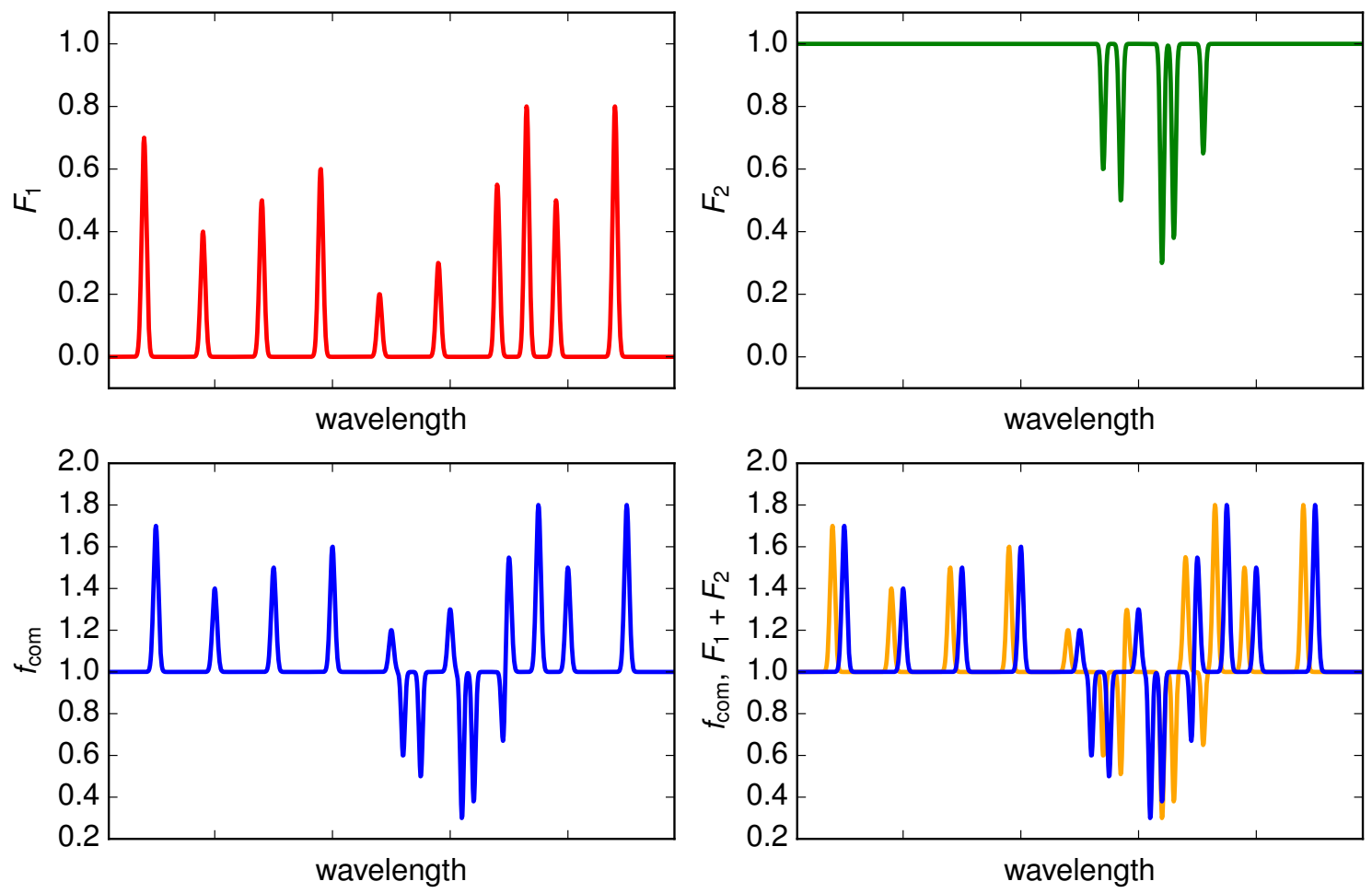

Figure 2.5: Illustration of the modified algorithm. Top panels: template spectra $F_{1}$ and $F_{2}$ (representing discharge spectrum and absorption gas cell spectrum, respectively). Lower panel (left): A recorded spectrum of the two combined sources $f_{\text {com. }}$. Lower panel (right): A recorded spectrum of the two combined sources $f_{\text {com }}$ (blue curve) and the sum of the two templates (yellow curve). Notice the small wavelength offset (in different directions) between $f_{\text {com }}$ and $F_{1}+F_{2}$. The algorithm tries to match the spectra by shifting the two templates.

The goal is to minimize the sum of $R$ over all $N$ observed wavelengths (datapoints) in a least-squares sense, i.e., we try to minimize

$$
\chi^{2}=\sum_{i=1}^{N} \omega_{i}\left(R\left[\lambda_{i}, \hat{\alpha}\right]\right)^{2} .
$$

The weights $\omega_{i}$ are discussed at the end of this section in more detail. Equation 2.20 is a non-linear expression for the difference $R$ as a function of the parameters $\hat{\alpha}$. We do a 
Taylor expansion up to the linear terms evaluated at a value $\hat{\alpha}_{(0)}$ :

$$
\begin{aligned}
R[\lambda, \hat{\alpha}] & \approx R\left[\lambda, \hat{\alpha}_{(0)}\right] \\
& +\left.\frac{\partial R}{\partial \alpha_{1, \mathrm{v}}}\right|_{\lambda, \hat{\alpha}_{(0)}}\left(\alpha_{1, \mathrm{v}}-\alpha_{1, \mathrm{v}(0)}\right)+\left.\frac{\partial R}{\partial \alpha_{1,0}}\right|_{\lambda, \hat{\alpha}_{(0)}}\left(\alpha_{1,0}-\alpha_{1,0}(0)\right. \\
& +\left.\frac{\partial R}{\partial \alpha_{1,1}}\right|_{\lambda, \hat{\alpha}_{(0)}}\left(\alpha_{1,1}-\alpha_{1,1_{(0)}}\right)+\left.\frac{\partial R}{\partial \alpha_{2, \mathrm{v}}}\right|_{\lambda, \hat{\alpha}_{(0)}}\left(\alpha_{2, \mathrm{v}}-\alpha_{2, \mathrm{v}(0)}\right) \\
& +\left.\frac{\partial R}{\partial \alpha_{2,0}}\right|_{\lambda, \hat{\alpha}_{(0)}}\left(\alpha_{2,0}-\alpha_{2,0}(0)\right)+\left.\frac{\partial R}{\partial \alpha_{2,1}}\right|_{\lambda, \hat{\alpha}_{(0)}}\left(\alpha_{2,1}-\alpha_{2,1}(0)\right),
\end{aligned}
$$

with the derivatives

$$
\begin{aligned}
\left.\frac{\partial R}{\partial \alpha_{1, \mathrm{v}}}\right|_{\lambda, \hat{\alpha}_{(0)}} & =\left.\frac{\partial F_{1}\left[\alpha_{1, \mathrm{v}} \lambda\right]}{\partial\left(\alpha_{1, \mathrm{v}} \lambda\right)} \frac{\partial\left(\alpha_{1, \mathrm{v}} \lambda\right)}{\partial \alpha_{1, \mathrm{v}}} \cdot\left(\alpha_{1,0}+\alpha_{1,1}\left(\lambda-\lambda_{c}\right)\right)\right|_{\lambda, \hat{\alpha}_{(0)}} \\
& =\left.\lambda \frac{\partial F_{1}\left[\alpha_{1, \mathrm{v}} \lambda\right]}{\partial\left(\alpha_{1, \mathrm{v}} \lambda\right)} \cdot\left(\alpha_{1,0}+\alpha_{1,1}\left(\lambda-\lambda_{c}\right)\right)\right|_{\lambda, \hat{\alpha}_{(0)}}, \\
\left.\frac{\partial R}{\partial \alpha_{1,0}}\right|_{\lambda, \hat{\alpha}_{(0)}} & =\left.F_{1}\left[\alpha_{1, \mathrm{v}} \lambda\right]\right|_{\lambda, \hat{\alpha}_{(0)}}, \\
\left.\frac{\partial R}{\partial \alpha_{1,1}}\right|_{\lambda, \hat{\alpha}_{(0)}} & =\left.F_{1}\left[\alpha_{1, \mathrm{v}} \lambda\right]\left(\lambda-\lambda_{c}\right)\right|_{\lambda, \hat{\alpha}_{(0)}}, \\
\left.\frac{\partial R}{\partial \alpha_{2, \mathrm{v}}}\right|_{\lambda, \hat{\alpha}_{(0)}} & =\left.\frac{\partial F_{2}\left[\alpha_{2, \mathrm{v}} \lambda\right]}{\partial\left(\alpha_{2, \mathrm{v}} \lambda\right)} \frac{\partial\left(\alpha_{2, \mathrm{v}} \lambda\right)}{\partial \alpha_{2, \mathrm{v}}} \cdot\left(\alpha_{2,0}+\alpha_{2,1}\left(\lambda-\lambda_{c}\right)\right)\right|_{\lambda, \hat{\alpha}_{(0)}} \\
\left.\frac{\partial R}{\partial \alpha_{2,0}}\right|_{\lambda, \hat{\alpha}_{(0)}} & =\left.F_{2}\left[\alpha_{2, \mathrm{v}} \lambda\right]\right|_{\lambda, \hat{\alpha}_{(0)}}, \\
\left.\frac{\partial R}{\partial \alpha_{2,1}}\right|_{\lambda, \hat{\alpha}_{(0)}} & =F_{2}\left[\alpha_{2, \mathrm{v}} \lambda\right]\left(\lambda-\alpha_{c, \mathrm{v}} \lambda\right] \\
& =\left.\left(\alpha_{2,0}+\alpha_{2,1}\left(\lambda-\lambda_{c}\right)\right)\right|_{\lambda, \hat{\alpha}_{(0)}},
\end{aligned}
$$

Using the Taylor-expanded expression for $R$ and $\delta \hat{\alpha}=\hat{\alpha}-\hat{\alpha}_{(0)}$, we can rewrite the merit function (Eq. 2.21):

$$
\begin{array}{r}
\chi^{2}=\sum_{i}^{N} \omega_{i}\left(R\left[\lambda_{i}, \hat{\alpha}_{(0)}\right]+\left.\frac{\partial R}{\partial \alpha_{1, \mathrm{v}}}\right|_{\lambda, \hat{\alpha}_{(0)}} \delta \alpha_{1, \mathrm{v}}+\left.\frac{\partial R}{\partial \alpha_{1,0}}\right|_{\lambda, \hat{\alpha}_{(0)}} \delta \alpha_{1,0}+\left.\frac{\partial R}{\partial \alpha_{1,1}}\right|_{\lambda, \hat{\alpha}_{(0)}} \delta \alpha_{1,1}\right. \\
\left.+\left.\frac{\partial R}{\partial \alpha_{2, \mathrm{v}}}\right|_{\lambda, \hat{\alpha}_{(0)}} \delta \alpha_{2, \mathrm{v}}+\left.\frac{\partial R}{\partial \alpha_{2,0}}\right|_{\lambda, \hat{\alpha}_{(0)}} \delta \alpha_{2,0}+\left.\frac{\partial R}{\partial \alpha_{2,1}}\right|_{\lambda, \hat{\alpha}_{(0)}} \delta \alpha_{2,1}\right)^{2} .
\end{array}
$$


The next step is to minimize the merit-function. This done by calculating derivatives with respect to the increments $\delta \hat{\alpha}$ and setting them to zero:

$$
\begin{aligned}
& \frac{\partial \chi^{2}}{\partial \delta \alpha_{1, \mathrm{v}}}=\left.2 \sum_{i=1}^{N} \omega_{i} R\left[\lambda_{i}, \hat{\alpha}\right] \frac{\partial R}{\partial \alpha_{1, \mathrm{v}}}\right|_{\lambda, \hat{\alpha}_{(0)}} \stackrel{!}{=} 0 \\
& \frac{\partial \chi^{2}}{\partial \delta \alpha_{1,0}}=\left.2 \sum_{i=1}^{N} \omega_{i} R\left[\lambda_{i}, \hat{\alpha}\right] \frac{\partial R}{\partial \alpha_{1,0}}\right|_{\lambda, \hat{\alpha}_{(0)}} \stackrel{!}{=} 0 \\
& \frac{\partial \chi^{2}}{\partial \delta \alpha_{1,1}}=\left.2 \sum_{i=1}^{N} \omega_{i} R\left[\lambda_{i}, \hat{\alpha}\right] \frac{\partial R}{\partial \alpha_{1,1}}\right|_{\lambda, \hat{\alpha}_{(0)}} \stackrel{!}{=} 0 \\
& \frac{\partial \chi^{2}}{\partial \delta \alpha_{2, \mathrm{v}}}=\left.2 \sum_{i=1}^{N} \omega_{i} R\left[\lambda_{i}, \hat{\alpha}\right] \frac{\partial R}{\partial \alpha_{2, \mathrm{v}}}\right|_{\lambda, \hat{\alpha}_{(0)}} \stackrel{!}{=} 0 \\
& \frac{\partial \chi^{2}}{\partial \delta \alpha_{2,0}}=\left.2 \sum_{i=1}^{N} \omega_{i} R\left[\lambda_{i}, \hat{\alpha}\right] \frac{\partial R}{\partial \alpha_{2,0}}\right|_{\lambda, \hat{\alpha}_{(0)}} \stackrel{!}{=} 0 \\
& \frac{\partial \chi^{2}}{\partial \delta \alpha_{2,1}}=\left.2 \sum_{i=1}^{N} \omega_{i} R\left[\lambda_{i}, \hat{\alpha}\right] \frac{\partial R}{\partial \alpha_{2,1}}\right|_{\lambda, \hat{\alpha}_{(0)}} \stackrel{!}{=} 0
\end{aligned}
$$

We can solve this system of six equations for the increments $\delta \hat{\alpha}$ by using the matrix notation (the evaluation points and dependencies are dropped for better readability):

$$
\begin{aligned}
& \left(\begin{array}{llllllll}
\sum \frac{\partial R}{\partial \alpha_{1, \mathrm{v}}} \frac{\partial R}{\partial \alpha_{1, \mathrm{v}}} & \sum \frac{\partial R}{\partial \alpha_{1, \mathrm{v}}} \frac{\partial R}{\partial \alpha_{1,0}} & \sum \frac{\partial R}{\partial \alpha_{1, \mathrm{v}}} \frac{\partial R}{\partial \alpha_{1,1}} & \sum \frac{\partial R}{\partial \alpha_{1, \mathrm{v}}} \frac{\partial R}{\partial \alpha_{2, \mathrm{v}}} & \sum \frac{\partial R}{\partial \alpha_{1, \mathrm{v}}} \frac{\partial R}{\partial \alpha_{2,0}} & \sum \frac{\partial R}{\partial \alpha_{1, \mathrm{v}}} \frac{\partial R}{\partial \alpha_{2,1}} \\
\sum \frac{\partial R}{\partial \alpha_{1,0}} \frac{\partial R}{\partial \alpha_{1, \mathrm{v}}} & \sum \frac{\partial R}{\partial \alpha_{1,0}} \frac{\partial R}{\partial \alpha_{1,0}} & \sum \frac{\partial R}{\partial \alpha_{1,0}} \frac{\partial R}{\partial \alpha_{1,1}} & \sum \frac{\partial R}{\partial \alpha_{1,0}} \frac{\partial R}{\partial \alpha_{2, \mathrm{v}}} & \sum \frac{\partial R}{\partial \alpha_{1,0}} \frac{\partial R}{\partial \alpha_{2,0}} & \sum \frac{\partial R}{\partial \alpha_{1,0}} \frac{\partial R}{\partial \alpha_{2,1}} \\
\sum \frac{\partial R}{\partial \alpha_{1,1}} \frac{\partial R}{\partial \alpha_{1, \mathrm{v}}} & \sum \frac{\partial R}{\partial \alpha_{1,1}} \frac{\partial R}{\partial \alpha_{1,0}} & \sum \frac{\partial R}{\partial \alpha_{1,1}} \frac{\partial R}{\partial \alpha_{1,1}} & \sum \frac{\partial R}{\partial \alpha_{1,1}} \frac{\partial R}{\partial \alpha_{2, \mathrm{v}}} & \sum \frac{\partial R}{\partial \alpha_{1,1}} \frac{\partial R}{\partial \alpha_{2,0}} & \sum \frac{\partial R}{\partial \alpha_{1,1}} \frac{\partial R}{\partial \alpha_{2,1}} \\
\sum \frac{\partial R}{\partial \alpha_{2, \mathrm{v}}} \frac{\partial R}{\partial \alpha_{1, \mathrm{v}}} & \sum \frac{\partial R}{\partial \alpha_{2, \mathrm{v}}} \frac{\partial R}{\partial \alpha_{1,0}} & \sum \frac{\partial R}{\partial \alpha_{2, \mathrm{v}}} \frac{\partial R}{\partial \alpha_{1,1}} & \sum \frac{\partial R}{\partial \alpha_{2, \mathrm{v}}} \frac{\partial R}{\partial \alpha_{2, \mathrm{v}}} & \sum \frac{\partial R}{\partial \alpha_{2, \mathrm{v}}} \frac{\partial R}{\partial \alpha_{2,0}} & \sum \frac{\partial R}{\partial \alpha_{2, \mathrm{v}}} \frac{\partial R}{\partial \alpha_{2,1}} \\
\sum \frac{\partial R}{\partial \alpha_{2,0}} \frac{\partial R}{\partial \alpha_{1, \mathrm{v}}} & \sum \frac{\partial R}{\partial \alpha_{2,0}} \frac{\partial R}{\partial \alpha_{1,0}} & \sum \frac{\partial R}{\partial \alpha_{2,0}} \frac{\partial R}{\partial \alpha_{1,1}} & \sum \frac{\partial R}{\partial \alpha_{2,0}} \frac{\partial R}{\partial \alpha_{2, \mathrm{v}}} & \sum \frac{\partial R}{\partial \alpha_{2,0}} \frac{\partial R}{\partial \alpha_{2,0}} & \sum \frac{\partial R}{\partial \alpha_{2,0}} \frac{\partial R}{\partial \alpha_{2,1}} \\
\sum \frac{\partial R}{\partial \alpha_{2,1}} \frac{\partial R}{\partial \alpha_{1, \mathrm{v}}} & \sum \frac{\partial R}{\partial \alpha_{2,1}} \frac{\partial R}{\partial \alpha_{1,0}} & \sum \frac{\partial R}{\partial \alpha_{2,1}} \frac{\partial R}{\partial \alpha_{1,1}} & \sum \frac{\partial R}{\partial \alpha_{2,1}} \frac{\partial R}{\partial \alpha_{2, \mathrm{v}}} & \sum \frac{\partial R}{\partial \alpha_{2,1}} \frac{\partial R}{\partial \alpha_{2,0}} & \sum \frac{\partial R}{\partial \alpha_{2,1}} \frac{\partial R}{\partial \alpha_{2,1}}
\end{array}\right) \\
& \left(\begin{array}{l}
\delta \alpha_{1, \mathrm{v}} \\
\delta \alpha_{1,0} \\
\delta \alpha_{1,1} \\
\delta \alpha_{2, \mathrm{v}} \\
\delta \alpha_{2,0} \\
\delta \alpha_{2,1}
\end{array}\right)=\left(\begin{array}{l}
-\sum R \frac{\partial R}{\partial \alpha_{1, \mathrm{v}}} \\
-\sum R \frac{\partial R}{\partial \alpha_{1,0}} \\
-\sum R \frac{\partial R}{\partial \alpha_{1,1}} \\
-\sum R \frac{\partial R}{\partial \alpha_{2, \mathrm{v}}} \\
-\sum R \frac{\partial R}{\partial \alpha_{2,0}} \\
-\sum R \frac{\partial R}{\partial \alpha_{2,1}}
\end{array}\right)
\end{aligned}
$$

This is a simple matrix equation of the form $\underline{A} \cdot \underline{\delta \alpha}=\underline{b}$ that can be solved for the vector $\underline{\delta \alpha}$ with many standard software packages (e.g., Numpy's linalg. solve for Python). The calculation is repeated iteratively and the free parameters are updated with the calculated increments $\hat{\alpha}_{\text {new }}=\hat{\alpha}_{(0)}+\delta \hat{\alpha}$. The iterative process is stopped as soon as the increment falls below a threshold. I use the criterion $\delta v_{i}=\delta \alpha_{i, \mathrm{v}} \cdot c<10^{-4} \mathrm{~m} / \mathrm{s}$ for $i=\{1,2\}$, and the algorithm usually needs only a few iterations $(<6)$ to converge. The RV shift of each template can be calculated from the final parameter $\alpha_{\mathrm{v}}$ using the following formula (use 
$\alpha_{1, \mathrm{v}}$ or $\alpha_{2, \mathrm{v}}$ accordingly):

$$
\lambda_{\mathrm{obs}}=\lambda_{\mathrm{em}} \alpha_{\mathrm{v}} \stackrel{\mathrm{Eq} .2 .17}{=} \lambda_{\mathrm{em}} \cdot\left(1+\frac{v}{c}\right) \quad \Rightarrow v=\left(\alpha_{\mathrm{v}}-1\right) \cdot c
$$

The errors on the free parameters can be calculated from the covariance matrix which is the inverse of matrix $A$ :

$$
\sigma\left(\alpha_{k}\right)=\sqrt{\left(A^{-1}\right)_{k k}} \cdot c
$$

To obtain meaningful uncertainties from Eq. 2.23 through error propagation, the weights $\omega_{i}$ have to be properly chosen. The algorithm uses information from three different spectra, $F_{1}, F_{2}$ and $f_{\text {com }}$, and therefore the weights need to account for the noise $\sigma(f)$ from all three spectra $\left(f=\left\{F_{1}, F_{2}, f_{\text {com }}\right\}\right)$. I use $\omega=\left(\sigma\left(F_{1}\right)^{2}+\sigma\left(F_{2}\right)^{2}+\sigma\left(f_{\text {com }}\right)^{2}\right)^{-1}$ because the measurements are taken independently of each other. For spectra recorded with the FTS, the weights are the same at all wavelengths and $\sigma(f)$ is determined as described in Sect. 3.4.1. The measurement errors from the FTS spectra are normally distributed as can, e.g., be seen from the right panel in Fig. 3.5 (Sect. 3.4.1). This is a requirement so that the covariance matrix of the least-squares minimization has a clear quantitative interpretation (Press et al., 2002). Anglada-Escudé \& Butler (2012) note that correlations between parameters are ignored when using Eq. 2.23 to calculate the errors, but that it still provides good estimates for the formal precision of the results. A Markov Chain Monte Carlo (MCMC) calculation was used to sample the multidimensional probability space in order to determine correlations between parameters and to visualize the parameter space (e.g., Goodman \& Weare, 2010). The MCMC simulations were carried out with the software package emcee for Python ${ }^{1}$. The projections of samples in the multidimensional parameter space showed little correlation of $\alpha_{1, \mathrm{v}}$ and $\alpha_{2, \mathrm{v}}$ with the other parameters. The simulations were done for a few of the spectra of the two time series presented in Sect. 5.3. In all of these cases, the final parameters matched the results from the least-squares procedure at least at the significant digits. The calculated errors for the parameters were slightly smaller when determined from the MCMC simulation. The MCMC calculations verify that the results from the least-squares algorithm are reliable. This conclusion, drawn from the MCMC simulations using a few spectra of each time series, can be safely assumed to apply to all the spectra that have been analyzed with the least-squares technique, because the spectra of a time series are all very similar. A practical advantage of the least-squares algorithm is the shorter computation time: it takes only a few seconds to reach convergence, while a full MCMC simulation takes several hours for each spectrum ${ }^{2}$.

\subsection{Radial velocity information content of a spectrum}

The shape and the signal-to-noise ratio of a spectrum set a limit to the achievable precision of Doppler shift measurements. Connes (1985) first presented the calculation of the velocity

\footnotetext{
${ }^{1}$ http://dan.iel.fm/emcee/

${ }^{2}$ Calculations were run on an Intel Xeon X5680 @ 3.33GHz computer; the exact computation time for MCMC depends on the number of samples and the number of parallel threads.
} 
uncertainty corresponding to a certain spectral profile and photon count. Later, the photon noise limit for Doppler shift measurements was discussed again in the context of exoplanet detection by Butler et al. (1996) and Bouchy et al. (2001). The presentations in these papers focus on stellar spectra that are recorded with echelle spectrographs. This includes that the dominating noise is assumed to be photon noise. However, spectra recorded with an FTS exhibit a different noise behavior (see Sect. 3.4.1 and, e.g., Davis et al., 2001). The following derivation follows the principle steps used by the above mentioned authors, but presents the final equation for the velocity uncertainty in quantities that can be directly measured from the FTS spectra (Eq. 2.27). This equation is used in Sect. 5.3.3 to calculate the RV information content in spectra of the MIP lamp.

We consider a noise-free template spectrum with intensity $f_{0, i}$ and wavelength $\lambda_{i}$ at a sampling point labeled $i$. A second spectrum is taken at a later epoch under similar conditions, but this time the spectrum contains random noise. It will reveal a change in intensity $\left(f_{0, i}\right.$ becomes $\left.f_{i}\right)$ at a certain sampling point, either from statistical noise, a change in radial velocity, or a combination of both. For a small wavelength shift, the change in intensity can be written as

$$
f_{0, i}-f_{i} \approx \frac{\partial f_{i}}{\partial \lambda_{i}} \cdot \Delta \lambda_{i}=\frac{\partial f_{i}}{\partial \lambda_{i}} \cdot \frac{v_{i}}{c} \lambda_{i},
$$

where Eq. 2.18 was used for the Doppler shift in the last step (Bouchy et al., 2001). We rearrange the above equation to obtain a new expression for the (relative) Doppler shift:

$$
v_{i}=\frac{f_{0, i}-f_{i}}{\lambda_{i} \cdot\left(\partial f_{i} / \partial \lambda_{i}\right)} \cdot c .
$$

The radial velocity is measured through a change in intensity for a given sampling point $i$. We can calculate the uncertainty of the velocity through error propagation, assuming that $f_{i}$ is the only noise-affected quantity:

$$
\sigma\left(v_{i}\right)=\sqrt{\left(\sigma\left(f_{i}\right) \cdot \frac{\partial v_{i}}{\partial f_{i}}\right)^{2}}=\frac{\sigma(f)}{\lambda_{i} \cdot\left|\partial f_{i} / \partial \lambda_{i}\right|} \cdot c .
$$

For measurements with the FTS, the noise in the spectrum can be expected to be uniformly distributed over all wavelengths (see Sect. 3.4.1). Therefore, the index $i$ on $\sigma(f)$ is dropped. For FTS spectra, a correction for zero-filling as described in Sect. 3.4.1 needs to be considered in order to obtain the correct weight for each data point from $\sigma(f)$. Next, we calculate the weighted mean of the velocities from all $N$ data points in the wavelength range under investigation. The weighted mean is calculated in the following way

$$
v=\frac{\sum_{i}^{N} v_{i} w_{i}}{\sum_{i}^{N} w_{i}},
$$

with the weights given by $w_{i}=1 / \sigma\left(v_{i}\right)^{2}$ (Drosg, 2009). For the following calculation, we will also need the partial derivative:

$$
\frac{\partial v}{\partial v_{i}}=\frac{w_{i}}{\sum_{j}^{N} w_{j}} .
$$


We calculate the uncertainty of the velocity measurement over all wavelengths through Gaussian error propagation of the uncertainties in the underlying individual data points:

$$
\begin{aligned}
\sigma(v) & =\sqrt{\sum_{i}^{N}\left(\frac{\partial v}{\partial v_{i}} \cdot \sigma\left(v_{i}\right)\right)^{2}} \stackrel{\text { Eq. } .225}{=} \sqrt{\sum_{i}^{N}\left(\frac{w_{i}^{2}}{\left(\sum_{j}^{N} w_{j}\right)^{2}} \cdot \frac{1}{w_{i}}\right)} \\
& =\sqrt{\frac{\sum_{i}^{N} w_{i}}{\left(\sum_{j}^{N} w_{j}\right)^{2}}}=\sqrt{\frac{1}{\sum_{i}^{N} w_{i}}} \\
& =\frac{1}{\sqrt{\sum_{i}^{N} \frac{1}{\sigma^{2}\left(v_{i}\right)}}} .
\end{aligned}
$$

We substitute Eq. 2.24 into Eq. 2.26 and obtain the final expression for the uncertainty of radial velocity measurements due to the shape and the noise of the spectrum:

$$
\sigma(v)=c \cdot\left(\sqrt{\sum_{i=1}^{N}\left(\frac{\lambda_{i} \cdot\left(\partial f_{i} / \partial \lambda_{i}\right)}{\sigma(f)}\right)^{2}}\right)^{-1}
$$

Consequently, $\sigma(v)$ decreases if the spectral lines are sharp (large derivatives) and the uncertainties on the individual data points are small. Furthermore, more data points and therefore more spectral lines increase the precision. For these reasons, precision radial velocity measurements are best conducted at a high resolution that allows to record sharp spectral lines and with spectrographs that can record a large spectral range. 



\section{Fourier transform spectroscopy}

Contents

3.1 Introduction $\ldots \ldots \ldots \ldots \ldots \ldots \ldots \ldots \ldots \ldots \ldots$

3.2 Principles of Fourier transform spectroscopy . . . . . . . . . 32

3.2 .1 The ideal instrument . . . . . . . . . . . . . . . . . . 32

3.2.2 Limited optical path difference and apodization . . . . . . . . 34

3.2 .3 Finite size of entrance aperture . . . . . . . . . . . . 37

3.2 .4 Phase shift . . . . . . . . . . . . . . . . . . 40

3.3 The Bruker IFS 125 HR $\ldots \ldots \ldots \ldots \ldots$

3.4 Analysis of FTS spectra $\ldots \ldots \ldots \ldots \ldots$

3.4.1 Determination of noise level . . . . . . . . . . . . . . 44

3.4.2 A multi-profile fit algorithm for emission line spectra . . . . . . 46

\subsection{Introduction}

The Institut für Astrophysik in Göttingen operates a Bruker IFS 125 HR Fourier transform spectrometer (FTS). Its basic design is that of a Michelson interferometer. An FTS does not directly measure the light's intensity as a function of wavelength, but observations are made in a conjugate space (Fourier space) in form of an interferogram. The spectrum is later calculated from the interferogram with a Fourier transform. The IFS $125 \mathrm{HR}$ is the primary instrument for the spectral analysis of the microwave-induced plasma lamp.

Advantages of an FTS are the high spectral resolution and the integrated wavelength scale which is generated in reference to an internal helium-neon laser. The achievable resolution is in principal determined by the maximum optical path difference (OPD). The OPD is the difference in the path lengths of the two beams in the interferometer. With a maximum OPD of $\Delta=208 \mathrm{~cm}^{-1}$, the Göttingen FTS can achieve a nominal resolution of $\Delta \tilde{\nu}=1 / \Delta=0.0048 \mathrm{~cm}^{-1}$. The frequency scale of a spectrum recorded with an FTS is usually given in wavenumbers $\tilde{\nu}$ with the unit inverse centimeters $\left(\mathrm{cm}^{-1}\right)$. The conversion to wavelengths $\lambda$, which are more common for visible and near-infrared spectroscopy in astronomy, is given by:

$$
\tilde{\nu}\left[\mathrm{cm}^{-1}\right]=\frac{10^{7} \frac{\mathrm{nm}}{\mathrm{cm}}}{\lambda[\mathrm{nm}]} .
$$


The terms "wavenumbers" and "wavelength" are often used synonymously in this thesis, e.g., the designation "wavelength scale" is used for FTS spectra, although they are usually displayed as a function of wavenumber.

In Sect. 3.2, I summarize principles of Fourier transform spectroscopy. The FTS began operation at the Institut für Astrophysik only a few weeks before the start of the work for this thesis, and some practical aspects that have been learned since then are included in Sect. 3.2. Extensive literature on Fourier transform spectroscopy is available, e.g., in the textboooks by Chamberlain (1979), Brault (1985), Kauppinen \& Partanen (2001), Davis et al. (2001), Wartewig (2003), and Griffiths \& de Haseth (2007). The design of the Göttingen FTS is presented in Sect. 3.3. In Sect. 3.4, I discuss the determination of noise in the FTS spectra and outline a modeling algorithm that I developed to determine parameters of emission lines.

\subsection{Principles of Fourier transform spectroscopy}

\subsubsection{The ideal instrument}

Figure 3.1 shows a schematic drawing of a Michelson interferometer. At this point, a perfectly collimated beam of monochromatic light with a single wavelength $\lambda_{0}=1 / \tilde{\nu}_{0}$ is assumed. We can derive an expression for the interference signal after the light has passed through the instrument (see also the textbooks listed in Sect. 3.1). The incident light can be described as a plane electromagnetic wave

$$
A_{\text {in }}=A_{0}\left(\tilde{\nu}_{0}\right) \cdot e^{-2 i \pi \tilde{\nu}_{0} x},
$$

where $A_{0}$ is the maximum amplitude and $x$ is a point along the direction of travel of the wave. One part of the electromagnetic wave transmits through the beamsplitter, gets reflected at mirror M1 and then gets reflected at the beamsplitter. It has the amplitude $A_{1}$ after passing through the instrument and covering the optical path length $x_{1}$. The second part of the wave gets first reflected at the beamsplitter, gets reflected at mirror M2, and then transmits through the beamsplitter. It has the amplitude $A_{2}$ after passing through the interferometer and covering the optical path length $x_{2}$. At this point, we assume that there are no losses at the optical components. The beamsplitter shall have a reflectance of $R$ and a transmittance of $T$, with $R+T=1$. The recombined wave $A\left(\tilde{\nu}_{0}\right)$ is the sum of the two individual waves:

$$
\begin{aligned}
A\left(\tilde{\nu}_{0}\right) & =A_{1}\left(\tilde{\nu}_{0}\right)+A_{2}\left(\tilde{\nu}_{0}\right) \\
& =\sqrt{R} \cdot \sqrt{T} \cdot A_{0}\left(\tilde{\nu}_{0}\right) \cdot e^{-2 i \pi \tilde{\nu}_{0} x_{1}}+\sqrt{R} \cdot \sqrt{T} \cdot A_{0}\left(\tilde{\nu}_{0}\right) \cdot e^{-2 i \pi \tilde{\nu}_{0} x_{2}} \\
& =\sqrt{R} \cdot \sqrt{T} \cdot A_{0}\left(\tilde{\nu}_{0}\right) \cdot\left(e^{-2 i \pi \tilde{\nu}_{0} x_{1}}+e^{-2 i \pi \tilde{\nu}_{0} x_{2}}\right)
\end{aligned}
$$

The square root of $T$ and $R$ is used here because we operate on the amplitude of the electric field (see, e.g., Demtröder, 2006). On the detector, we measure intensities that are 


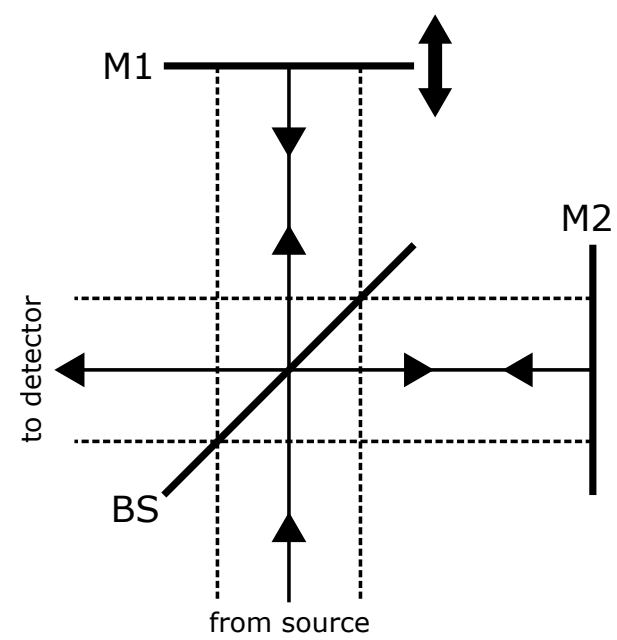

Figure 3.1: Schematic drawing of a Michelson interferometer. Dashed lines are the outer rays of the collimated beam through the instrument. BS: beamsplitter, M1: movable mirror, M2: fixed mirror.

proportional to the squared amplitude. Using $I_{0}=A_{0}^{2}$, we can write for the intensity:

$$
\begin{aligned}
I^{\prime} \propto|A|^{2} & =A A^{*} \\
& =R T I_{0}\left(\tilde{\nu}_{0}\right) \cdot\left(e^{-2 i \pi \tilde{\nu}_{0} x_{1}}+e^{-2 i \pi \tilde{\nu}_{0} x_{2}}\right)\left(e^{+2 i \pi \tilde{\nu}_{0} x_{1}}+e^{+2 i \pi \tilde{\nu}_{0} x_{2}}\right) \\
& =R T I_{0}\left(\tilde{\nu}_{0}\right) \cdot\left(1+e^{2 i \pi \tilde{\nu}_{0}\left(x_{2}-x_{1}\right)}+e^{-2 i \pi \tilde{\nu}_{0}\left(x_{2}-x_{1}\right)}+1\right) \\
& =2 R T I_{0}\left(\tilde{\nu}_{0}\right) \cdot\left[1+\cos \left(2 \pi \tilde{\nu}_{0}\left(x_{2}-x_{1}\right)\right)\right] .
\end{aligned}
$$

Next, we introduce the optical path difference $\delta=x_{2}-x_{1}$, and assume that the beamsplitter provides $50 \%$ reflection and $50 \%$ transmission, $R=T=0.5$ :

$$
I^{\prime}(\delta) \propto \frac{1}{2} I_{0}\left(\tilde{\nu}_{0}\right)\left[1+\cos \left(2 \pi \tilde{\nu}_{0} \delta\right)\right] .
$$

This is the intensity of the wave at the detector, also called interference record (Griffiths \& de Haseth, 2007, Sect. 2.2). It is composed of a constant component and an alternating term. For spectroscopy, usually only the alternating term is considered. After subtraction of the constant component, we obtain the interferogram

$$
I(\delta) \propto \frac{1}{2} I\left(\tilde{\nu}_{0}\right) \cdot \cos \left(2 \pi \tilde{\nu}_{0} \delta\right) .
$$

In practice, there are losses throughout the instrument due to optical efficiency and detector response. We take these effects into account by replacing $I$ with $S$ :

$$
I(\delta)=S\left(\tilde{\nu}_{0}\right) \cdot \cos \left(2 \pi \tilde{\nu}_{0} \delta\right)
$$

If we measure a light source that emits not only one wavenumber $\tilde{\nu}_{0}$, we need to adjust 
Eq. 3.1 by integrating over all wavenumbers:

$$
I(\delta)=\int_{-\infty}^{\infty} S(\tilde{\nu}) \cos (2 \pi \tilde{\nu} \delta) \mathrm{d} \tilde{\nu}
$$

This is one part of a Fourier transform pair. The spectrum $S(\tilde{\nu})$ is calculated from the interferogram through the inverse transform:

$$
S(\tilde{\nu})=\int_{-\infty}^{\infty} I(\delta) \cos (2 \pi \tilde{\nu} \delta) \mathrm{d} \delta
$$

In theory, a spectrum can be calculated with infinitely high resolution and accuracy using Eq. 3.3. In reality, this is not possible because of practical limitations, including the following three: (i) the optical path difference is limited by the finite scan length of the traveling mirror; (ii) the beam through the interferometer is not perfectly collimated because of an extended source; and (iii) the exact position of the zero path difference is often not known introducing a phase shift. In the following sections, I discuss some aspects of these three limitations with applications to the Göttingen FTS . A much more detailed and in-depth discussion can again be found in the already mentioned textbooks on Fourier transform spectroscopy (see Sect. 3.1).

\subsubsection{Limited optical path difference and apodization}

A real-world interferometer has a finite size and therefore a finite scan length limiting the achievable resolution. As a result, the theoretical spectrum of a monochromatic light source is no longer a sharp $\delta$-peak, but the spectral line has a non-zero line width. In addition, the truncation of the interferogram leads to artifacts in the spectrum in form of sidelobes near the spectral lines. A way to reduce these sidelobes is the application of an apodization function which will be discussed later in this section.

Mathematically, the finite maximum optical path difference $\Delta$ modifies the integration limits in Eq. 3.3:

$$
S_{\Delta}(\tilde{\nu})=\int_{-\Delta}^{\Delta} I(\delta) \cdot \cos (2 \pi \tilde{\nu} \delta) \mathrm{d} \delta
$$

Instead of modifying the integration limits, we can also multiply the interferogram $I(\delta)$ with a boxcar function $D(\delta) \equiv \Pi(\delta / 2 \Delta)$ having the following properties:

$$
D(\delta)= \begin{cases}1, & \text { if }|\delta| \leq \Delta \\ 0, & \text { if }|\delta|>\Delta\end{cases}
$$

The convolution theorem states that the Fourier transform of two multiplied functions is equal to the convolution of the two individually Fourier transformed functions (e.g., Woan, 
2000). The Fourier transform of the interferogram $I(\delta)$ is of course the spectrum $S(\tilde{\nu})$, and the Fourier transform of the boxcar function is designated with $F(\tilde{\nu})$ :

$$
S_{\Delta}(\tilde{\nu})=\int_{-\infty}^{\infty} I(\delta) \cdot D(\delta) \cdot \cos (2 \pi \tilde{\nu} \delta) \mathrm{d} \delta=S(\tilde{\nu}) * F(\tilde{\nu})
$$

It can be shown (e.g., Woan, 2000) that $F(\tilde{\nu})$ is a sinc function:

$$
F(\tilde{\nu})=2 \Delta \frac{\sin (2 \pi \tilde{\nu} \Delta)}{2 \pi \tilde{\nu} \Delta}=2 \Delta \operatorname{sinc}(2 \pi \tilde{\nu} \Delta) .
$$

The sinc function $F(\tilde{\nu})$ is the natural instrumental line shape (ILS) function of the FTS, i.e., the broadened line profile of a monochromatic light source due to the limited optical path difference. This is inevitably connected to a fundamental question in spectroscopy, which is the definition of a resolution criterion: how far do two spectral features need to be separated in wavelength to be individually measurable? Different resolution criteria, such as Rayleigh or full width at half maximum (FWHM), have been defined (Griffiths \& de Haseth, 2007, Sect. 2.3). The width of the ILS becomes a crucial parameter in this context. To calculate the FWHM of the sinc function, we find the wavenumber $\tilde{\nu}_{\frac{1}{2}}$ where the profile has half the flux compared to its center at wavenumber $\tilde{\nu}_{0}$ :

$$
F\left(\tilde{\nu}_{\frac{1}{2}}\right)=2 \Delta \operatorname{sinc}\left(2 \pi \tilde{\nu}_{\frac{1}{2}} \Delta\right) \stackrel{!}{=} \frac{2 \Delta}{2}=\frac{F\left(\tilde{\nu}_{0}\right)}{2} \quad \Rightarrow \quad \operatorname{sinc}\left(2 \pi \tilde{\nu}_{\frac{1}{2}} \Delta\right)=\frac{1}{2} .
$$

We solve numerically for $\tilde{\nu}_{\frac{1}{2}}$ and obtain $\tilde{\nu}_{\frac{1}{2}}= \pm 0.60335 /(2 \Delta)$. Consequently, the FWHM of the sinc profile is given by:

$$
F W H M_{\text {sinc }}=2 \cdot\left|\tilde{\nu}_{\frac{1}{2}}\right|=\frac{0.60335}{\Delta} .
$$

The left panel of Fig. 3.2 shows one sinc function centered around wavenumber $\tilde{\nu}_{0}$ (blue curve) and a second sinc function shifted by $F W H M_{\text {sinc }}=0.6 / \Delta$. The dashed red curve represents the sum of the two sinc profiles. One can see that the two peaks are not resolved if they are separated by their FWHM, as the red dashed curve does only show one peak at its center. In addition, one can infer from the left panel of Fig. 3.2 that the convolution with a sinc function does not only broaden a spectral line but does also introduce strong sidelobes. These are artefacts from the abrupt truncation of the interferogram at its end and do not have a physical meaning. The sidelobes can be reduced by multiplying the interferogram with an apodization function. The apodization modifies the interferogram to ensure a smoother transition toward zero at its ends. The case where no apodization is applied is also called boxcar apodization because of the shape of function $D(\delta)$.

The triangular function is a special apodization profile when working with the Bruker IFS 125 HR FTS. The Fourier transform of the triangular function is the function $\operatorname{sinc}^{2}(\pi \tilde{\nu} \Delta)$ and its profile is shown in the right panel of Fig. 3.2 (e.g., Woan, 2000). The sidelobes are much smaller for this type of apodization and the two spectral lines can be resolved if separated by $F W H M_{\text {sinc }^{2}}=0.9 / \Delta$. For the Bruker IFS 125 HR FTS, the resolution and the maximum OPD are related through

$$
\Delta=\frac{0.9}{(\Delta \tilde{\nu})_{\text {OPUS }}},
$$



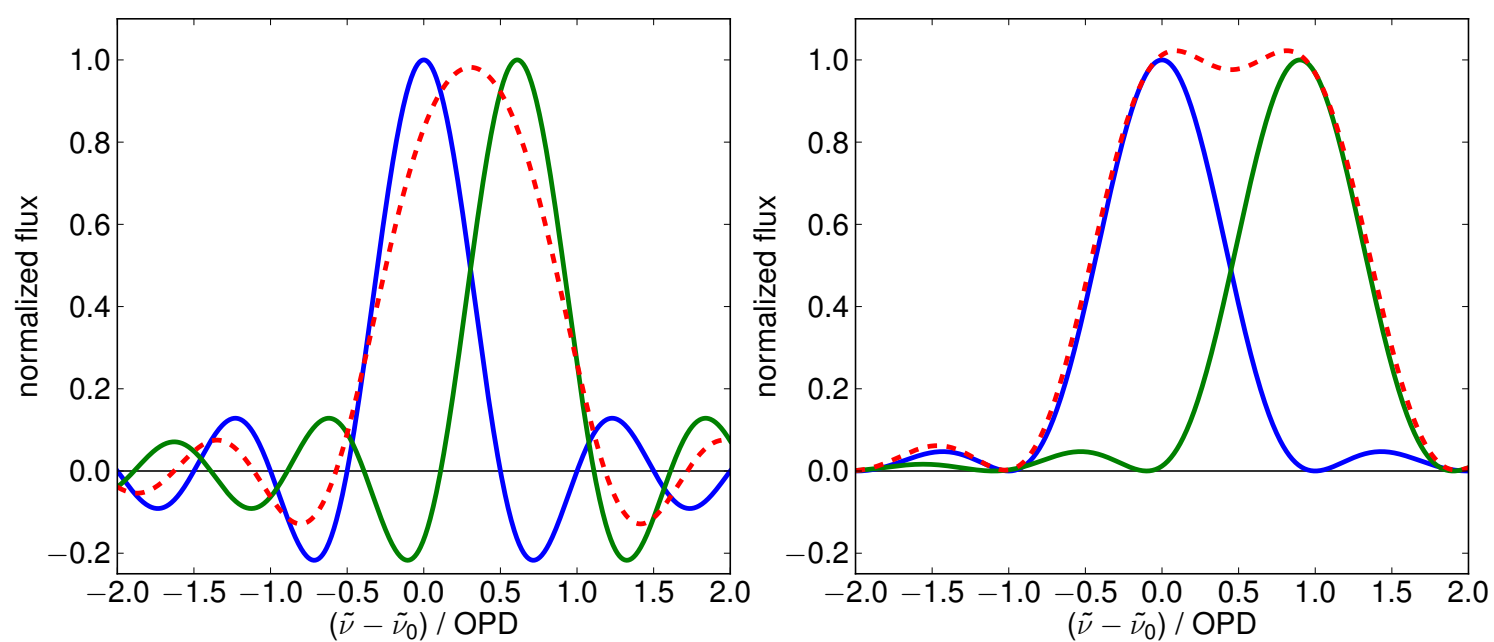

Figure 3.2: Left panel (boxcar apodization): The function $\operatorname{sinc}(2 \pi \tilde{\nu} \Delta)$ centered at wavenumber $\tilde{\nu}_{0}$ (blue curve) and the same function shifted by one FWHM (green curve). The red dashed curve is the sum of the two sinc profiles. Right panel (triangular apodization): Same as left panel, but with function $\operatorname{sinc}^{2}(\pi \tilde{\nu} \Delta)$.

where $(\Delta \tilde{\nu})_{\text {OPUS }}$ is the resolution set in the OPUS software which controls the FTS (Bruker Optics, private communication and IFS $120 \mathrm{HR}$ user's manual). The resolution $(\Delta \tilde{\nu})_{\text {OPUS }}$ corresponds to the FWHM of the ILS using a triangular apodization. The reference to the triangular apodization has an additional motivation: most conventional spectrographs use an entrance slit that determines the amount of light entering the instrument, and the diffraction pattern behind a slit follows the profile of a sinc ${ }^{2}$ function (see, e.g., Demtröder, 2006). Beside the triangular apodization, other apodization function have been developed and Table 3.1 lists the methods that are available in the OPUS software. The usage of apodization comes at the cost of a wider ILS function and a reduced spectral resolution. The stronger the apodization, i.e., the more the sidelobes are suppressed, the higher the degradation of the resolution. The selection of a particular type of apodization is therefore always a compromise between these two aspects. In practice, the observer specifies a desired resolution in the OPUS software. This resolution assumes the usage of the triangular apodization function. If a different apodization is used, the corresponding observed resolution needs to be calculated by comparing the change in the width of the ILS function. The last column in Table 3.1 lists the conversion factors between $(\Delta \tilde{\nu})$ OPUS and the observed resolution $\Delta \tilde{\nu}$ for each apodization function.

The nominal resolution $\Delta \tilde{\nu}=1 / \Delta$ is generally used if the exact value is not required (Griffiths \& de Haseth, 2007). It is a good approximation and independent of the applied apodization and resolution criterion. 


\begin{tabular}{lcc}
\hline \hline apodization & FWHM & $\Delta \tilde{\nu} /(\Delta \tilde{\nu})$ OPUS \\
\hline Boxcar / none & $0.61 / \Delta$ & 0.68 \\
Triangular & $0.9 / \Delta$ & 1 \\
Four points / Trapezoidal & between & boxcar and triangular \\
Happ-Genzel & $1.1 / \Delta$ & 1.23 \\
Blackman-Harris, 3-term & $0.92 / \Delta$ & 1.02 \\
Blackman-Harris, 4-term & $1.4 / \Delta$ & 1.52 \\
Norton-Beer, weak & $0.73 / \Delta$ & 0.81 \\
Norton-Beer, medium & $0.85 / \Delta$ & 0.95 \\
Norton-Beer, strong & $0.98 / \Delta$ & 1.08 \\
\hline
\end{tabular}

Table 3.1: Apodization functions provided by the OPUS software with the FWHM of the respective Fourier transforms (ILS functions). The last column gives the conversion factor between the resolution value in the OPUS software and the actual FWHM of the ILS. Reference: Bruker Optics, IFS 120 HR user's manual; see also Wartewig (2003).

\subsubsection{Finite size of entrance aperture}

A perfectly collimated beam was assumed for the discussion of the ideal spectrometer in Sect. 3.2.1. However, only a point source can in theory be perfectly collimated, while an extended source will always slowly diverge. For the FTS, the size of the light source is set by the circular entrance aperture. It has a finite opening which introduces two effects: the wavelength scale is shifted and the resolution is degraded. Both effects are discussed in this section.

The light rays in a not perfectly collimated beam travel different paths through the interferometer and interfere after different optical path differences. If $\alpha$ is the divergence half angle of the beam and $l$ is the distance that the moving mirror travels, the generalized optical path difference is given by the following relation (Brault, 1985, Sect. 3.2):

$$
\delta=2 l \cos (\alpha)
$$

This results in $\delta=2 l$ for the central ray traveling on the optical axis $(\alpha=0)$ and a smaller OPD for the outer rays $(\alpha>0)$. The diverging beam creates a light cone with a solid angle $\Omega$ that is related to the divergence half angle through the relation $\Omega=\pi \alpha^{2}=\pi r^{2} / f^{2}$. Here, $r$ is the radius of the cone at the (focal) distance $f$, and $\alpha$ is assumed to be small enough to apply the small angle approximation. The solid angle extends to $\Omega_{\max }$ for the outer rays.

For simplicity, a monochromatic light source is assumed for the following calculation. We 
substitute Eq. 3.7 into Eq. 3.1 and integrate over the solid angle:

$$
\begin{aligned}
I_{\Omega} & =\int_{0}^{\Omega_{\max }} S\left(\tilde{\nu}_{0}\right) \cdot \cos \left(2 \pi \tilde{\nu}_{0} \cdot 2 l \cos (\alpha)\right) \mathrm{d} \Omega \\
& =\int_{0}^{\Omega_{\max }} S\left(\tilde{\nu}_{0}\right) \cdot \cos \left(2 \pi \tilde{\nu}_{0} \cdot 2 l\left(1-\frac{\Omega}{2 \pi}\right)\right) \mathrm{d} \Omega \\
& =S\left(\tilde{\nu}_{0}\right) \cdot \Omega_{\max } \operatorname{sinc}\left(\frac{\tilde{\nu}_{0} l \Omega_{\max }}{2}\right) \cdot \cos \left(2 \pi \tilde{\nu}_{0} \cdot 2 l\left(1-\frac{\Omega_{\max }}{4 \pi}\right)\right) .
\end{aligned}
$$

See Brault (1985, Sect. 3.2 and Appendix I) for the evaluation of the integral. In case of a polychromatic light source, one would still need to integrate over all wavenumbers. The modified interferogram $I_{\Omega}$ contains additional terms compared to the interferogram given in Eq. 3.1. These terms represent the two already mentioned effects: the modified argument of the cosine function is related to the wavelength shift and the additional factor before the cosine function changes the resolution. I discuss both effect now in more detail.

For a diverging beam, the measured wavenumbers $\tilde{\nu}_{\text {measured }}$ are shifted compared to the wavenumbers $\tilde{\nu}_{\text {center }}$ that would be recorded from the central ray traveling at the optical axis of the instrument. The result is a redshift of the spectrum, i.e., the wavenumbers appear to be smaller than the true values:

$$
\tilde{\nu}_{\text {measured }}=\tilde{\nu}_{\text {center }}\left(1-\frac{\Omega_{\max }}{4 \pi}\right) .
$$

The internal HeNe laser is another component that adds a wavenumber shift. It does not take the exact same path through the interferometer as the science light beam, and also has a different beam divergence (Griffiths \& de Haseth, 2007, Sect. 2.6). The total wavenumber shift is linear and can be corrected with a single correction factor $\kappa$ :

$$
\tilde{\nu}_{\text {corrected }}=\tilde{\nu}_{\text {measured }}(1+\kappa)
$$

Salit et al. (2004) showed that a single multiplicative correction factor $\kappa$ is accurate to $6 \cdot 10^{-9}$ (corresponds to about $2 \mathrm{~m} / \mathrm{s}$ ) for their FTS at optical wavelengths. For the Göttingen FTS, wavelength stability measurements with the discharge spectra (compare Sect. 5.3) have also not revealed a noticeable wavelength dependency.

The expression for the interferogram $I_{\Omega}$ also includes a new factor $\Omega_{\max } \operatorname{sinc}\left(\tilde{\nu} l \Omega_{\max } / 2\right)$ as compared to Eq. 3.1. This results in a circular fringe pattern at the detector plane, even for a monochromatic light source as it can be seen in Fig. 2.13 in Griffiths \& de Haseth (2007). With other words, the divergence angle of the beam is mapped into radii of the fringe pattern (see also Fig. 5.2 in Davis et al., 2001). In the spectral domain, the Fourier transform of the function $\operatorname{sinc}\left(\tilde{\nu}_{0} l \Omega_{\max } / 2\right)$ becomes the boxcar function $\Pi\left(2 \pi \tilde{\nu} / \Omega_{\max } \tilde{\nu}_{0}\right)$ with a width of $w=\Omega_{\max } \tilde{\nu}_{0} / 2 \pi$ (compare Eqs. 3.4 and 3.5). Thus, the ILS is further broadened through a convolution with a boxcar function. To minimize this degradation in resolution, only light from the central fringe must be recorded at the detector. It can be shown (Davis et al., 2001, Sect. 5.2) that this can be achieved by limiting the diameter of the entrance aperture: there is a maximum aperture diameter $d_{\max }$ for a chosen 
resolving power $R=\tilde{\nu} / \Delta \tilde{\nu}$, or a maximum wavenumber $\tilde{\nu}_{\max }$ that can be recorded without a significant loss of resolution for a given diameter $d$ :

$$
d_{\max }=f \sqrt{\frac{8}{R}} \quad \Leftrightarrow \quad \tilde{\nu}_{\max }=\Delta \tilde{\nu} \cdot \frac{8 f^{2}}{d^{2}} .
$$

I made the experience, that this criterion is used by the OPUS software to signal the user the maximum recommended aperture size for a given wavelength range.

We can estimate the fraction of the additional broadening of the ILS function relative to its width caused by the limited scan length. The maximum effect occurs for the largest aperture size at the largest wavenumber. We calculate the width of the boxcar function for the maximum aperture $d_{\max }$ :

$$
w=\frac{\tilde{\nu}_{0}}{2 \pi} \cdot \Omega_{\max }=\frac{\tilde{\nu}_{0}}{2 \pi} \cdot \frac{\pi r_{\max }^{2}}{f^{2}}=\frac{\tilde{\nu}_{0}}{2 \pi} \cdot \frac{\pi\left(f \sqrt{8 / R_{\max }} / 2\right)^{2}}{f^{2}}=\frac{\tilde{\nu}_{0}}{R_{\max }}=\Delta \tilde{\nu} \cdot \frac{\tilde{\nu}_{0}}{\tilde{\nu}_{\max }} .
$$

Thus, in the case of maximum aperture size, the maximum width of the boxcar function (at $\tilde{\nu}_{0}=\tilde{\nu}_{\max }$ ) is exactly one resolution element. In the case of no further apodization, this equals the width of the sinc function $w_{\max }=\Delta \tilde{\nu}=0.6 / \Delta$. I show in the left panel of Fig. 3.3 the additional broadening of the ILS function caused by the convolution with the boxcar function of width $w_{\max }$. The solid blue curve shows a sinc profile which is the ILS function for the boxcar apodization and the dashed blue curve is the result after convolution. The width of the line profile increases by $10.9 \%$. For comparison, if the boxcar has only half the width of the sinc function, the profile increases only by about $2 \%$ after convolution. The effect is therefore usually neglectable if the aperture size is limited as described above (Davis et al., 2001, Sect. 5.2).
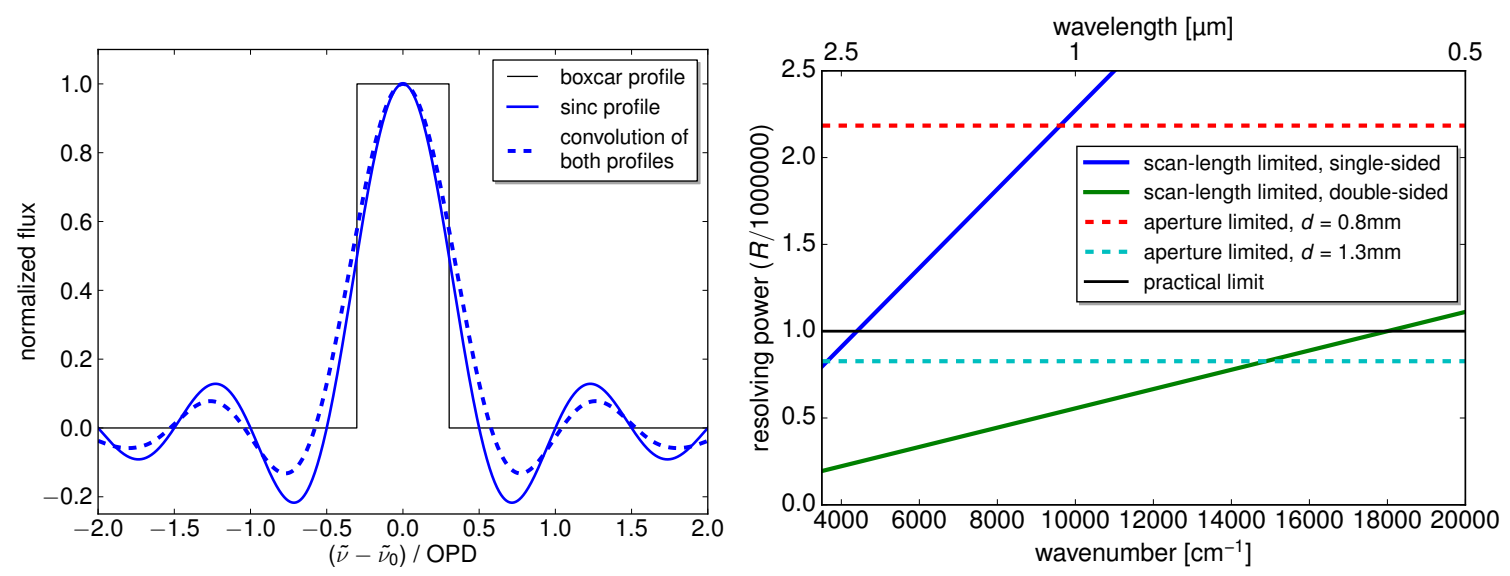

Figure 3.3: Left panel: Self-apodization due to finite entrance aperture. The solid blue curve represents a sinc profile. The dashed curve represents the profile after convolution with the boxcar function. Right panel: Maximum resolving power $R$ of the FTS as a function of wavenumber considering limited scan length and finite aperture.

For preparation of measurements, one often needs to know the maximum resolving power $R$ that can be achieved for a certain maximum OPD or a maximum aperture size. The 
right panel of Fig. 3.3 shows the achievable resolving power $R$ of the FTS as a function of wavenumber $\tilde{\nu}$. The solid blue and green lines mark the instrumentation limits that are caused by the finite scan lengths for two different modes of operation: singlesided and double-sided interferograms. The curves follow the relation $R=\tilde{\nu} / \Delta \tilde{\nu}$, with $\Delta \tilde{\nu}=0.0044 \mathrm{~cm}^{-1}$ for single-sided measurements and $\Delta \tilde{\nu}=0.018 \mathrm{~cm}^{-1}$ for double-sided measurements. The dashed red and cyan lines mark the limits due to a finite aperture for two different diameters of $d=0.8 \mathrm{~mm}$ and $d=1.3 \mathrm{~mm}$, respectively (see Eq. 3.10). In practice, a resolving power above one million is hardly reached for the IFS $125 \mathrm{HR}$ (Bruker Optics, personal communication and instrument brochure).

\subsubsection{Phase shift}

Davis et al. (2001, p. 101) write about the phase: "We know that the true spectrum is real and positive, and we assume that it can be represented by a symmetric function and by a cosine transform of a symmetric interferogram. The sine transform representing the complex part is zero. The phase is zero. But in fact the measured interferogram is not a real symmetric function, because experimental, instrumental, and computational limitations introduce asymmetries into it." As a consequence, distortions in line shapes and other artifacts can occur in the spectrum if no phase correction is applied.

For example, a phase shift $\Phi$ is introduced if no sampling point of the interferogram coincides with the zero path difference. Instead, there is an offset $\phi$ in the OPD and Eq. 3.2 changes to

$$
I_{\Phi}(\delta)=\int_{-\infty}^{\infty} S(\tilde{\nu}) \cos (2 \pi \tilde{\nu}(\delta+\phi)) \mathrm{d} \tilde{\nu}=\int_{-\infty}^{\infty} S(\tilde{\nu}) \cos (2 \pi \tilde{\nu} \delta+\Phi) \mathrm{d} \tilde{\nu}
$$

This is an example for a phase that depends linearly on wavenumber, $\Phi=2 \pi \tilde{\nu} \phi$. Other sources of phase errors are electronic filters or unbalanced dispersion from optics in the interferometer which can cause a phase spectrum that includes higher orders, $\Phi=$ $A+B \tilde{\nu}+C \tilde{\nu}^{2}+\ldots$ (Davis et al., 2001, Sect. 7.3, Griffiths \& de Haseth, 2007, Sect. 2.5).

A simple method to create a real and positive spectrum from a symmetric interferogram is to calculate the modulus or power spectrum. However, this comes at the cost of increased noise. Phase corrections methods, such as the one introduced by Mertz, determine the phase angle and allow to correct for phase shifts. (Griffiths \& de Haseth, 2007, Sect. 4.3)

The best way to reduce artifacts in the spectrum from a phase shift, is the recording of a symmetric, double-sided interferogram (Davis et al., 2001, Sects. 7.5 and 7.7). All measurements for the work present in this thesis use double-sided interferograms with a maximum resolution of $(\Delta \tilde{\nu})_{\text {OPUS }}=0.018 \mathrm{~cm}^{-1}$ and Mertz phase correction.

\subsection{The Bruker IFS $125 \mathrm{HR}$}

In this section, I describe the design of the Bruker IFS 125 HR Fourier transform spectrometer that was used as the primary instrument for recording spectra of the microwave-induced 
plasma lamp. Figure 3.4 shows a picture of the FTS with the beam path inside the instrument.

The light enters the instrument through one of two entrance windows made from calcium fluoride $\left(\mathrm{CaF}_{2}\right.$, with a wedge of $\left.6 \mathrm{mrad}\right)$. A fiber connector and a collimating mirror are mounted in front of one entrance window (label 1 in Fig. 3.4). This "parallel port" is used for all measurements of the microwave-induced plasma lamp that are presented in this thesis. The other entrance window allows to send light directly from external emission sources into the instrument (2). A breadboard is mounted to the FTS to assemble small experimental setups (3). Alternatively, the FTS houses two internal lamps which cover the optical to mid-infrared spectral range (tungsten lamp and globar; 4). The light is focused onto the circular entrance aperture before it enters the interferometer chamber (5). The size of the aperture can be changed from 0.5 to $12.5 \mathrm{~mm}$ in 16 steps. In combination with the parallel port, the maximum size of the aperture is limited by the diameter of the optical fiber if the aperture is not to be underfilled: the optics of the parallel port magnify the size of the fiber image by a factor of 2.5.

Inside the interferometer chamber, a parabolic mirror (6) with a focal length of $f=418 \mathrm{~mm}$ collimates the light before it reaches the beamsplitter. Three beamsplitters are available covering different wavelength regimes from the visible to the near-infrared (7): Quartz, $\mathrm{CaF}_{2}$, and potassium bromide $(\mathrm{KBr})$. The beamsplitter is positioned with an angle of $30^{\circ}$ relative to the incident beam. This angle allows for a larger throughput at the same beamsplitter size compared to the classical $45^{\circ}$ design (Griffiths \& de Haseth, 2007, Sect. 5.1.1). The separated beams get reflected at two mirrors. These are cube-corner (retro-) reflectors that send the light back on a path parallel to the incident beam. In contrast to flat mirrors, this design compensates tilts in all three dimensions (Griffiths \& de Haseth, 2007, Sect. 5.2.1). One of the two mirrors is fixed (8), while the other one can move back and forward changing the optical path difference (9). The movable mirror is mounted on top of a carriage that rides on two steel rods and the carriage is moved on the rails by a tensioned steel cable. In addition, the mirror sits on a voice coil on the carriage. The movement of this additional drive is used for low-resolution measurements, and to compensate for velocity errors in the main drive during high-resolution scans (Griffiths \& de Haseth, 2007, Sect. 5.2.1). The moving mirror can travel a distance of about $104 \mathrm{~cm}$ from the zero path difference. The fixed mirror is located about $50 \mathrm{~cm}$ away from the beamsplitter to allow recording of double-sided, symmetric interferograms. To record a double-sided interferogram, the moving mirror extends the scan beyond the position of zero path difference towards the beamsplitter.

After reflection at the cube-corner mirrors, the two separate light beams recombine at the beamsplitter. The recombined light is focused on a circular exit aperture before it enters the detector chamber (10). By default, the diameter of the exit aperture is one setting larger than the aperture at the interferometer's entrance. The FTS is provided with four detectors designed from different semiconductor materials (11). The silicon (Si) and indium gallium arsenide (InGaAs) photodiodes are operated at room temperature, while the indium antimonide (InSb) and mercury cadmium telluride (MCT or $\mathrm{HgCdTe}$ ) detectors need to be cooled with liquid nitrogen. For the measurements in the near-infrared 
(down to $4000 \mathrm{~cm}^{-1}$ ) presented in this thesis, I use the InSb detector in combination with the $\mathrm{CaF}_{2}$ beamsplitter.

Light from a stabilized HeNe laser (SIOS SL-04) is coupled into the interferometer near the beamsplitter and its interference signal is measured at two photodiodes on both sides of the beamsplitter (12). The interference signal of the laser is used to trigger the analog-to-digital converter (ADC) connected to the detector in the detector chamber. This method ensures that the sampling points of the interferogram correspond to equal distances in the optical path difference. The right sampling of the interferogram is very important to avoid aliasing in the spectrum. Griffiths \& de Haseth (2007, Chap. 3) cover the sampling process in detail.

The instrument is controlled through Bruker's OPUS software package. The OPUS program was also used to carry out the Fourier transforms from interferograms to spectra. 


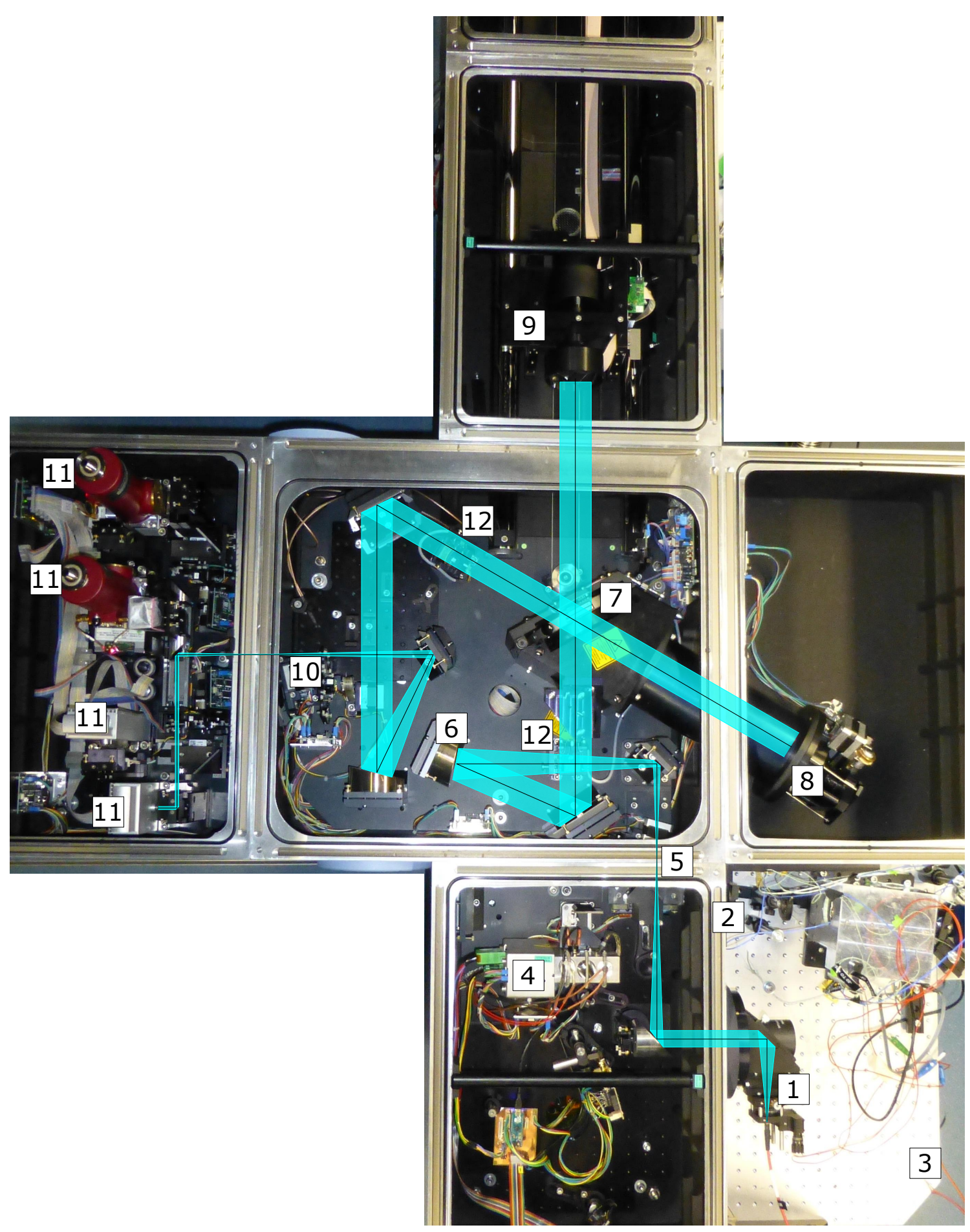

Figure 3.4: The Bruker IFS 125 HR Fourier Transform Spectrometer in the optics laboratory of the Institut für Astrophysik in Göttingen. The light path inside the instrument (from the fiber entrance port to the Silicon detector) is drawn into the picture. Refer to Sect. 3.3 for the numerical labels. 


\subsection{Analysis of FTS spectra}

In this section, I cover practical aspects regarding the calculation of the noise in the spectra and the determination of spectral line parameters. The methods for these two task have already been outlined in Boesch \& Reiners (2015). Here, the procedures are explained in more detail.

\subsubsection{Determination of noise level}

For grating spectrographs used in astronomy, the uncertainty in the flux measurement is often given as the square root of the photon count (photon noise). This is based on the fact that the photon count follows the discrete Poisson distribution (e.g., Appenzeller, 2013, Sect. 3.4 $)^{1}$. In Fourier transform spectroscopy the noise type in the spectrum is not as obvious, because we first record an interferogram and later calculate the spectrum using Fourier transform. Under the assumption of white noise in the interferogram, Davis et al. (2001, p. 121 and 122) come to the conclusion that "[...] the noise in the spectrum is uniformly distributed and does not depend on the local signal strength" and that "[...] the noise in the spectrum is proportional to the mean signal level only, in both absorption and emission". A study of the performance of an FTS with respect to photon noise and flicker noise was presented by Voigtman \& Winefordner (1987) with the following results: photon noise is uniformly distributed throughout the baseline of the spectrum; however, fluctuations of the source, or flicker noise, is frequency depended and is mapped into the surrounding wavelength region. These findings are also reported by Griffiths \& de Haseth (2007, Chap. 7). Analysis of emission spectra from the MIP lamp have shown that the noise in the baseline does not significantly change when measured in different spectral regions. I neglect intensity fluctuations of the source and assume that the noise in the interferogram is dominated by frequency independent detector noise and photon noise. Therefore, the error on the flux $f$ is constant over all wavenumbers in the spectrum, and a constant absolute noise level $\sigma_{\text {anl }}(f)$ can be defined as described below.

The recorded discharge spectra often exhibit densely-spaced emission lines over a large spectral range with almost no continuum where the noise can be measured. However, the spectra contain less and less lines toward wavenumbers smaller than $\approx 4200 \mathrm{~cm}^{-1}$, because the quartz optics (fibers, lenses) do not transmit light in this spectral range. Although there is no signal from the light source, this spectral region does have the same noise level as the rest of the spectrum. For example, I show in the left panel of Fig. 3.5 a discharge spectrum in the spectral range $4000-4500 \mathrm{~cm}^{-1}$ : the intensities of the spectral lines decrease towards smaller wavenumbers until there is no apparent signal above noise. It can also be seen from the spectral region $4000-4200 \mathrm{~cm}^{-1}$ that the baseline is not exactly centered around zero (orange line). A possible explanation for this offset can be found in the Fourier transform. An uncertainty in the constant term of the Fourier transform relates to an uncertainty of the intensity measurement in the interferogram at zero path difference. This

\footnotetext{
${ }^{1}$ The contributions of other noise sources (e.g., detector noise) need to be included for a more complete noise treatment.
} 
value is wavelength independent. I calculate the median of the flux values in the range $4000-4200 \mathrm{~cm}^{-1}$ and subtract this offset from the entire spectrum before further analysis. In the right panel of Fig. 3.5, I show a histogram with the distribution of the flux values from the wavenumber range $4000-4200 \mathrm{~cm}^{-1}$ (after subtraction of the baseline offset). I fit a Gaussian profile to the histogram and adopt the standard deviation of the Gaussian function as the absolute noise level $\sigma_{\text {anl }}$.
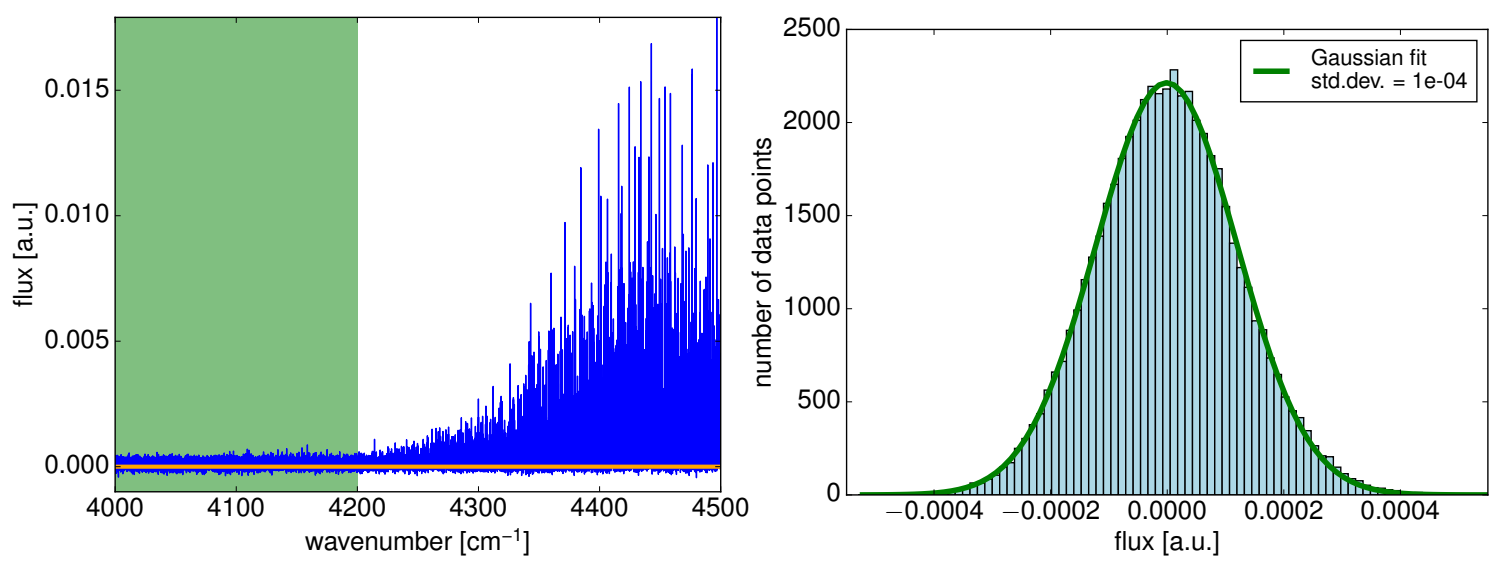

Figure 3.5: Left panel: Discharge spectrum in the wavenumber range $4000-4500 \mathrm{~cm}^{-1}$. The range shaded in green color is used to determine $\sigma_{a n l}$. Right panel: Histogram of the flux values in the spectral region 4000-4200 $\mathrm{cm}^{-1}$ after subtraction of the baseline offset (compare left panel). A Gaussian profile is fitted to the histogram (Boesch 83 Reiners, 2015, Fig. 3, adjusted).

The absolute noise level describes the scatter of the measured data points around the expected values. In Fourier transform spectroscopy, $\sigma_{\text {anl }}(f)$ does not necessarily give the correct weight for each data point to be used for error propagation. This is related to the procedure how the spectrum is calculated from the interferogram. First, the interferogram is sampled at $a_{\text {int }}$ independent points. The most commonly used technique to calculate the spectrum from the interferogram is the Cooley-Tukey algorithm, which is a very fast implementation of Fast Fourier Transform (FFT). The Cooley-Tukey algorithm works on datasets with $N_{\text {int }}=2^{n}$ data points, where $n$ is a positive integer (Press et al., 2002). To use all the recorded information of the interferogram, $N_{\text {int }}$ must be equal or larger than $a_{\text {int }}$. In practice, the number points in the interferogram does not equal a base 2 number and a method called zero-filling is applied: zeros are added at the end of the interferogram until its size equals a base 2 number. Different zero-filling factors can be selected in the OPUS software: A zero-filling factor of 2 adds just enough zero-valued points to reach an interferogram size of the next base 2 number; a zero-filling factor of 4 increases $n$ by 1 ; a zero-filling factor of 8 increases $n$ by 2 ; and so on. After FFT, the spectrum contains $N_{\text {int }}$ data points, and a higher value of $n$ leads to interpolation. The information that was originally recorded in $a_{\text {int }}$ independent sampling points in the interferogram is spread in the spectrum over $N_{\text {int }}$ points which are not all independent. The weight of each data point must therefore be corrected for the influence of zero-filling, and a correction factor can be derived from the definition of the standard deviation. The standard deviation for 
$N_{\text {int }}$ data points $f_{i}$ is defined as

$$
\sigma(f)=\sqrt{\frac{1}{N_{\text {int }}} \sum_{i=1}^{N_{\text {int }}}\left(f_{i}-\bar{f}\right)^{2}},
$$

where $\bar{f}$ is the mean of the sample of $f_{i}$ (Woan, 2000; for large $N_{\text {int }}$ there is no significant difference between dividing by $N_{\text {int }}$ or $N_{\text {int }}-1$ ). To take into account the $a_{\text {int }}$ independent points from the interferogram, Eq. 3.11 needs to be modified by multiplying the standard deviation with a correction factor $\sqrt{N_{\text {int }} / a_{\text {int }}}$. This way, we can calculate the weight of each data point $\sigma(f)$ by applying the correction factor to the absolute noise level: $\sigma(f)=\sqrt{N_{\text {int }} / a_{\text {int }}} \cdot \sigma_{\text {anl }}(f)$.

\subsubsection{A multi-profile fit algorithm for emission line spectra}

Creating a model of a recorded spectrum is among the first steps in the analysis of many spectral properties. For emission line spectra this task includes locating and fitting the emission peaks. The molecular spectra that are under investigation in this thesis cover a large spectral range of several thousand wavenumbers and exhibit thousands of spectral lines. Many of the lines are blended. There are two main challenges for the modeling procedure that arise from these properties. First, the large number of spectral lines increases the computing time. Second, the blended lines make it harder to determine the correct line properties. I developed a Python script tailored to determine line parameters of the discharge spectra recorded with the FTS. It can be highly parallelized to reduce the computing time and uses a superposition of line profiles to take into account the effect of blended lines. The algorithm is separated into two parts. It first locates the approximate positions of the peaks using the zero-crossings in the first derivative of the spectrum together with the corresponding peak intensities. Then, the line profiles are fitted to derive accurate line parameters, i.e., line positions, line intensities and line widths.

Spectral lines generally have the shape of a Voigt-profile (a convolution of a Gaussian and a Lorentzian profile) due to different line broadening mechanisms (see, e.g., Bernath, 2005). However, if pressure broadening can be neglected, compared to the other broadening mechanisms, a Gaussian profile is usually an adequate choice. I tested a Voigt profile for the fit function for different discharge spectra, but did not obtain better fit results as compared to a Gaussian profile. The Voigt profile is more sensitive at the wings of a line and, as consequence, is not as robust against noise or blends with low-intensity lines.

The routine to find and fit the emission lines uses the following steps:

1. Load the spectrum and subtract the offset value to center the baseline around zero. The offset value is calculated during the determination of the absolute noise level, as explained in Sect. 3.4.1.

2. Choose a minimum intensity threshold. Only peaks with an intensity above this threshold will be detected and included in the line fitting. This minimum peak intensity needs to be chosen manually and comes from experience. It should be a good compromise between including as many lines as possible in the fit and achieving 


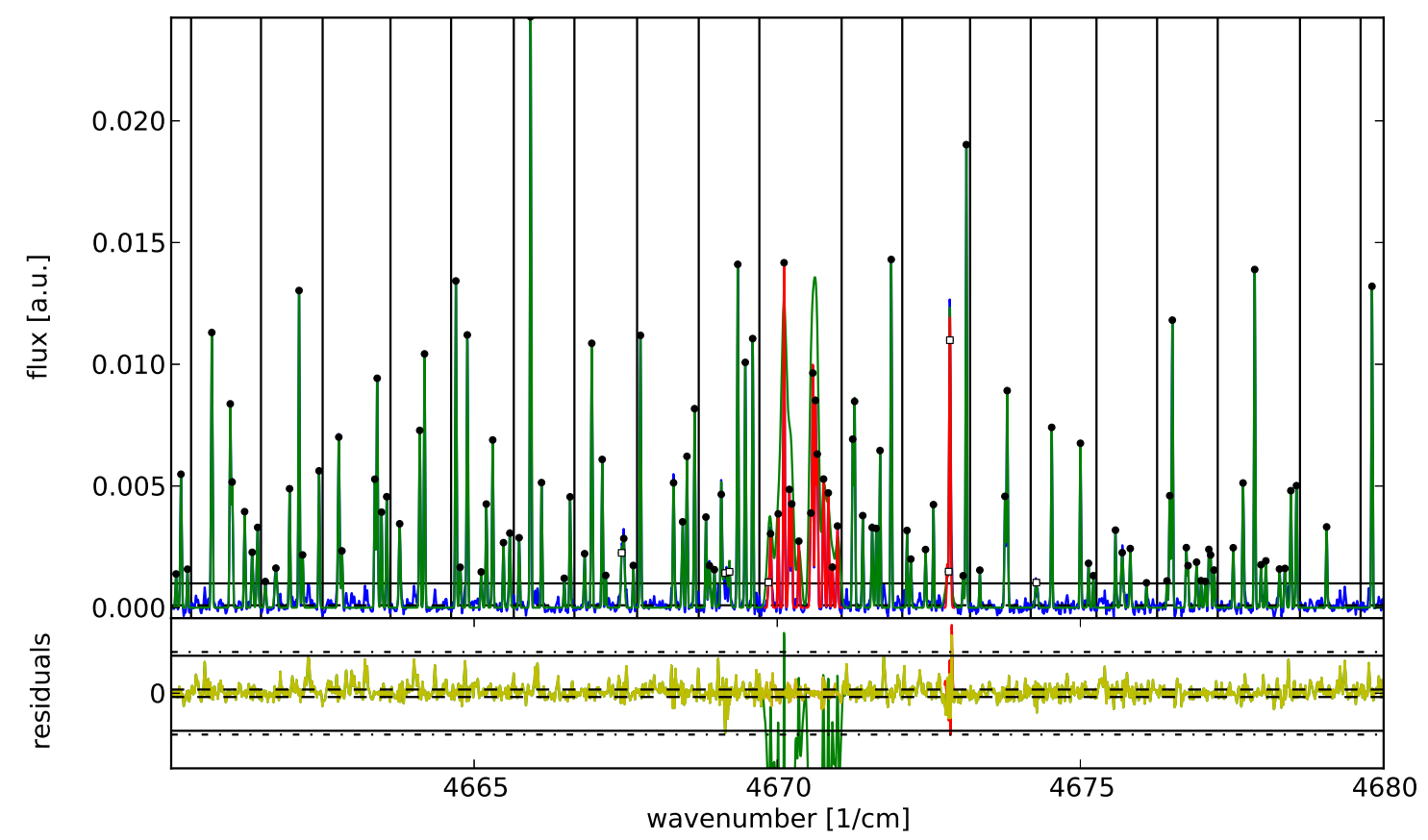

Figure 3.6: Spectrum of a nitrogen gas discharge (blue curve) and fits to the data (green and red curves). Illustration for the fit algorithm that is presented in the main text.

meaningful fit results for lines at low intensities. The minimum peak intensity can be chosen as a multiple of the absolute noise level $\sigma_{\text {anl }}$.

3. Calculate a cubic spline fit $f$ through the data points.

4. Calculate the derivative $f^{\prime}$ of the spline at each data point.

5. Search for zero-crossings in the derivative with a negative slope. Each zero-crossing yields two data points $\tilde{\nu}_{i}$ and $\tilde{\nu}_{i+1}\left(\tilde{\nu}_{i}<\tilde{\nu}_{i+1}\right)$ with $f^{\prime}\left(\tilde{\nu}_{i}\right)>0$ and $f^{\prime}\left(\tilde{\nu}_{i+1}\right)<0$.

6. Check, whether (i) $f\left(\tilde{\nu}_{i}\right)$ or $f\left(\tilde{\nu}_{i+1}\right)$ is larger than the minimum intensity threshold, and whether (ii) $f\left(\tilde{\nu}_{i}\right)$ or $f\left(\tilde{\nu}_{i+1}\right)$ is the maximum flux value within one resolution element. If both conditions are fulfilled, accept $\tilde{\nu}_{0}$ as peak position and $f\left(\tilde{\nu}_{0}\right)$ as peak intensity, with $f\left(\tilde{\nu}_{0}\right)=\max \left[f\left(\tilde{\nu}_{i}\right), f\left(\tilde{\nu}_{i+1}\right)\right]$. The resolution element equals the resolution value from the OPUS software times a factor that depends on the applied apodization (see Table 3.1). By definition, only one line can be resolved per resolution element.

7. Save the peak list.

8. Split the spectrum into bins. The spectral lines in the different bins will be modeled separately. The default bin size is $1 \mathrm{~cm}^{-1}$. The bin size is increased by one resolution element as long as a peak position is closer than three resolution elements to a boundary between two bins. This procedure minimizes boundary effect for the line fitting. 
9. Fit a superposition of Gaussian (or Voigt) profiles to the peaks in each bin. The results from step 6 are the start values for the fits. The results of the fits are the line center $\tilde{\nu}_{0}$, the line intensity $I$, the Gaussian width $\sigma_{\mathrm{G}}$ and, for the Voigt profile, the Lorentz parameter $\gamma$. The full width at half maximum (FWHM) of a line can be calculated using $F W H M=2 \sigma_{\mathrm{G}} \sqrt{2 \ln (2)}$ for a Gaussian profile or $F W H M=0.5346 \cdot 2 \gamma+\sqrt{0.2166 \cdot(2 \gamma)^{2}+\left(2 \sigma_{\mathrm{G}} \sqrt{2 \ln (2)}\right)^{2}}$ for a Voigt profile (Olivero \& Longbothum, 1977). The fitting procedure employs a weighted least-squares minimization (package mpfit for Python ${ }^{2}$ ). This task can be highly parallelized because the fits in the different spectral bins are independent of each other.

10. Model the entire spectrum by adding all line profiles with the line parameters determined in the previous step. Compare the recorded spectrum with the model by calculating the residuals.

11. Find outliers in the residuals. Residual points are considered outliers if their absolute values exceed a certain threshold, e.g., the minimum intensity threshold plus the absolute noise level. The closest spectral line position for each outlier is determined, and a line needs to be fitted again if an outlier falls within its FWHM. For strong lines, it can be difficult to keep the residuals below the above mentioned threshold. An addtional criterion is used to account for these cases: residuals also need to exceed a certain fraction of the line's peak intensity to be considered as outliers.

12. Determine new spectral ranges for the second round of fitting. The default fit range is based on the median FWHM of all lines $\left(F W H M_{\text {med }}\right)$ as calculated from the line parameters determined in step 9. The median FWHM of all lines is used, instead of a specific line's FWHM, because the latter could be a bad result from a failed fit. The fit range extends to $\pm 1.7 F W H M_{\text {med }}$ around the center of the line that needs to be fitted again. At this distance, a Gaussian profile has declined to $1 / e^{2}$ of its peak value and can be adequately modelled. The default fit range is extended until no line is closer than $1.7 F W H M_{\text {med }}$ to a boundary.

13. Fit a superposition of Gaussian (or Voigt) profiles to the peaks in each spectral region determined in the previous step. The start parameters for the fits are slightly changed from the first fit to reduce the chance of obtaining the same results.

14. In the spectral regions determined in step 12, calculate the weighted squared residuals $\left(\chi^{2}\right)$ using the results from the first and the second fit. Keep the line parameters that lead to a lower $\chi^{2}$.

15. Using the final line parameters, add all line profiles to model the entire spectrum, and check again for outliers (compare step 11).

16. Spectral lines are flagged for two cases: (i) if the fit did not converge properly within boundary conditions, and (ii) if the residuals are too large.

17. Save the line list with line position, peak intensity, Gaussian width, Lorentz parameter (=0 for Gaussian profile), FWHM, and flag indication.

\footnotetext{
${ }^{2}$ http://cars.uchicago.edu/software/python/mpfit.html
} 
In Fig. 3.6, some aspects of the algorithm are illustrated. The blue curve in the top panel is the experimental data (mostly covered by the green curve). The solid horizontal line represents the minimum intensity threshold. All peaks above this line are included in the modeling. The vertical lines are the bins for the first round of fitting. The green curve represents the model after the first fit, i.e., a superposition of Gaussian profiles with line parameters as determined from a multi-line fit in the separate bins. The residuals are plotted in the lower panel. The dashed line marks the absolute noise level, while the solid line represents again the minimum intensity threshold. The sum of these two values results in the dash-dotted line which marks the limit for outliers. We see in Fig. 3.6 that the residuals from the first fit exceed the dashed-dotted line in two spectral regions: at around $4670 \mathrm{~cm}^{-1}$ where the large residuals are clearly visible (green curve in the bottom panel); and at $4672.5 \mathrm{~cm}^{-1}$ where the green curve is hidden behind the yellow curve. These two regions are fitted again (red curve in top panel). For the spectral region around $4670 \mathrm{~cm}^{-1}$, the second fit delivers an improved result and the new line parameters are accepted. For the line at $4672.5 \mathrm{~cm}^{-1}$, the second fit does not improve the model and the parameters from the first fit are kept instead. The residuals for the final model are given by the yellow curve in the lower panel. All emission lines with a successful fit are marked with a full circle at the maximum peak position. The flagged lines (see step 16 above) are indicated by an open square. 



\section{The microwave-induced plasma lamp}

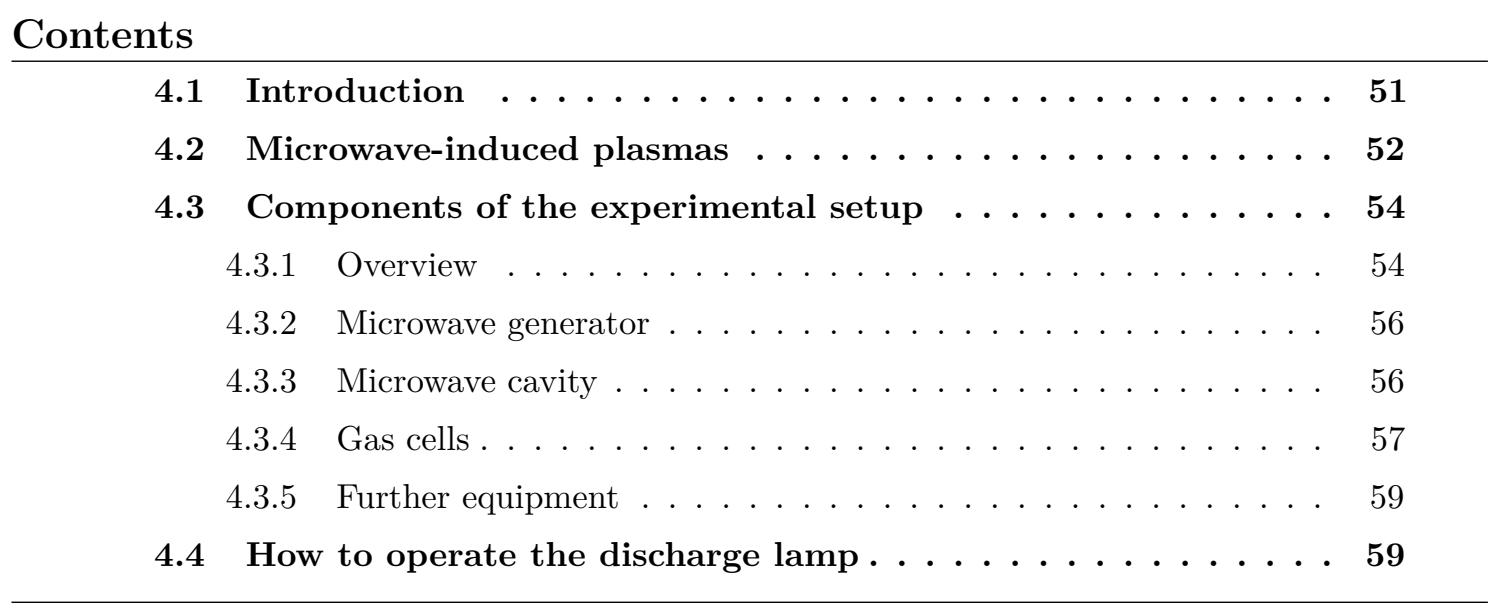

\subsection{Introduction}

In this chapter, I describe the experimental setup that I constructed during the time of the doctoral program. At the heart of the setup is a plasma source that can emit light from gas discharges. Additional equipment is used to feed the emitted light to the Fourier transform spectrometer (FTS) and for simultaneous wavelength calibration with a gas absorption cell. Light emission from molecules can be achieved in microwave (MW) plasmas that are frequently used by chemists in analytical spectrometry. Generally, there are two different kinds of microwave plasmas differentiated by the method of power transmission to the gas (Skogerboe \& Coleman, 1976). In the capacitively coupled plasma (CMP), the microwaves are guided to a coaxial electrode that is used to form a flamelike plasma at the tip of the electrode. A description and drawing of a CMP torch can be found in Cobine \& Wilbur (1951). A different technique, the microwave-induced plasma (MIP), uses an electrodeless resonant cavity to create the gas discharge inside a nonconductive tube.

The latter technique is used for the gas discharges in the presented experiment. The concept and properties of the MIP are introduced in Sect. 4.2. In Figure 4.1, I show an image of the first experimental setup with a gas discharge in the optics laboratory at the Institut für Astrophysik in Göttingen. The image provides a good overview over the components that are needed for an MIP. Since then, the experimental setup evolved into the state that is described in detail in Sect. 4.3. This chapter concludes with practical information on how to operate the MIP lamp (Sect. 4.4). 


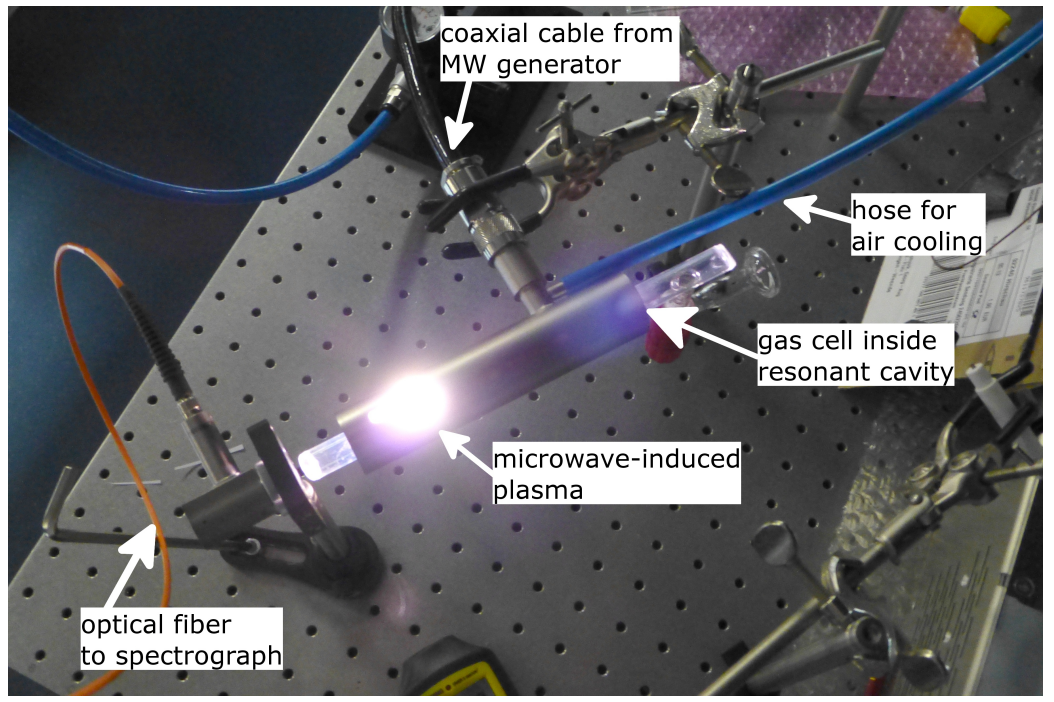

Figure 4.1: One of the first sustained discharges with a gas cell filled with helium (taken on 25 June 2013).

\subsection{Microwave-induced plasmas}

According to Rutscher (2001, p. 15), "Plasmas are quasi-neutral particle systems in the form of gaseous or fluid-like mixtures of free electrons and ions, frequently also containing neutral particles (atoms, molecules) [...]." They can be further divided into low- and high-pressure plasmas, and thermal- and non-thermal plasmas. An MIP is a non-thermal plasma and in general each species in the plasma has its own temperature (e.g., Jankowski \& Reszke, 2011, Sect. 1.4). It is characterized by comparatively low power, low running costs, and simple operation (Tschöpel, 1979, Sect. 8).

A typical frequency for MW applications is $2.45 \mathrm{GHz}$, because this frequency is cleared for commercial usage (Tschöpel, 1979). The mass production of commercial MW components operating at this frequency is a reason why the equipment comes at a relatively low cost. The power for the MIP is provided by a MW generator. The electromagnetic waves are guided from the generator via a coaxial cable to a resonant coaxial cavity. A non-conductive tube, e.g., made from quartz glass, passes through the cavity and contains the gas which is used to create the discharge. The cavity has the physical dimensions to support standing microwaves. The oscillating field in the cavity creates a strong electric field at a point where the tube intersects with the cavity. The flow of energy is shown in Fig. 4.2. To ignite the plasma, a short burst from a high-frequency generator (Tesla coil) is provided close to the discharge tube to create seed electrons in the gas. The free electrons are accelerated via the oscillating electric field until they have gained sufficient energy to cause excitation and ionization processes through collisions with gas atoms and molecules (Jankowski \& Reszke, 2011, Sect. 1.2). The molecules and atoms emit light when they relax from the excited state to a state of lower energy. For this kind of plasma generation, the gas is not in contact with any kind of electrode, but energy is transferred electrodeless via the 
interaction of the gas species with the electromagnetic field. This mode of operation gives reason to believe that the MIP lamp shows only little signs of aging because the gas does not interact with material from an electrode.

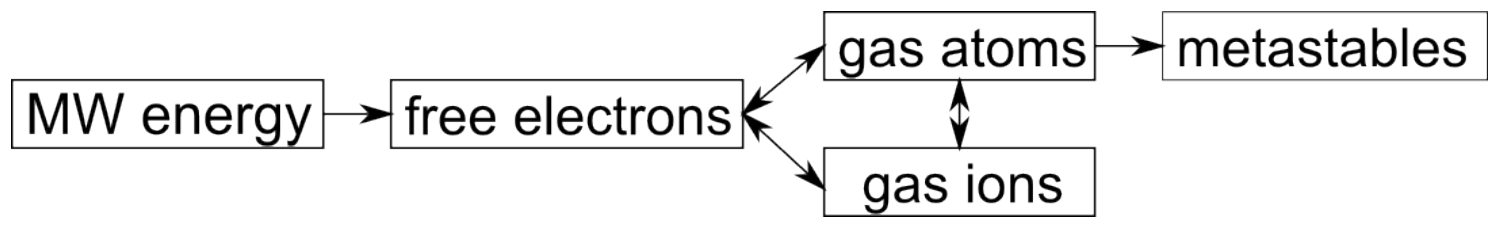

Figure 4.2: Flow of energy from microwave generator to the plasma. Adopted from Jankowski $\&$ Reszke (2011, Fig. 1.1).

The mean power absorbed by an electron from the microwave electric field is given by

$$
P=\frac{e^{2} E_{\max }^{2}}{2 m \nu} \cdot \frac{\nu^{2}}{\nu^{2}+\omega^{2}}
$$

where $e$ and $m$ are the charge and mass of the electron, respectively, $E_{\max }$ is the maximum field amplitude, $\omega$ is the field frequency, and $\nu$ is the collision frequency between the electron and the gaseous atoms (Jankowski \& Reszke, 2011, Sect. 1.2, Schmidt \& Conrads, 2001, Sect. 11.2). The latter is often called electron-neutral collision frequency because it measures the frequency of collisions between electrons and neutral particles. Sometimes the electron-electron and electron-ion collision frequencies are also included, but the collisions with these charges particles are relatively insignificant (Elghazaly et al., 2007). Because the electrons have a much lower mass as the other particles in the plasma, they are absorbing most of the energy from the microwaves (compare Eq. 4.1). The absorbed power $P$ is a function of the electron collision frequency $\nu$. We can find its maximum by calculating the derivative

$$
\begin{aligned}
\frac{\mathrm{d} P}{\mathrm{~d} \nu} & =\frac{e^{2} E_{\max }^{2}}{2 m} \cdot \frac{1 \cdot\left(\nu^{2}+\omega^{2}\right)-\nu \cdot 2 \nu}{\left(\nu^{2}+\omega^{2}\right)^{2}}=\frac{e^{2} E_{\max }^{2}}{2 m} \cdot \frac{\omega^{2}-\nu^{2}}{\left(\nu^{2}+\omega^{2}\right)^{2}} \\
& =\frac{e^{2} E_{\max }^{2}}{2 m} \cdot \frac{\omega-\nu}{\omega+\nu} \stackrel{!}{=} 0 .
\end{aligned}
$$

Solving for $\nu$ gives a maximum of $P$ at $\nu=\omega$. The electron-neutral collision frequency depends on the gas pressure and the nature of the gas (Schmidt \& Conrads, 2001, Sect. 11.3.4). According to Jankowski \& Reszke (2011), the optimum pressure for microwave plasma operation is about 4 Torr $(3 \mathrm{mbar})$ : low pressure means a smaller number of particles and a longer free path length between collisions which leads to high energies for the free electrons.

More information on microwave plasmas, including the historical development, can be found in the book by Jankowski \& Reszke (2011) and the references therein. Another overview of plasma excitation, including microwave-induced plasmas, is given by Tschöpel (1979). Different spectral light sources, including electrodeless discharge lamps, are covered in the book by Kirkbright \& Sargent (1974). The capacitively- and inductively-coupled plasmas (CCP and ICP) are also commonly found in analytical spectrometry, but operate at radio frequencies (e.g., Koch, 1985). 


\subsection{Components of the experimental setup}

\subsubsection{Overview}

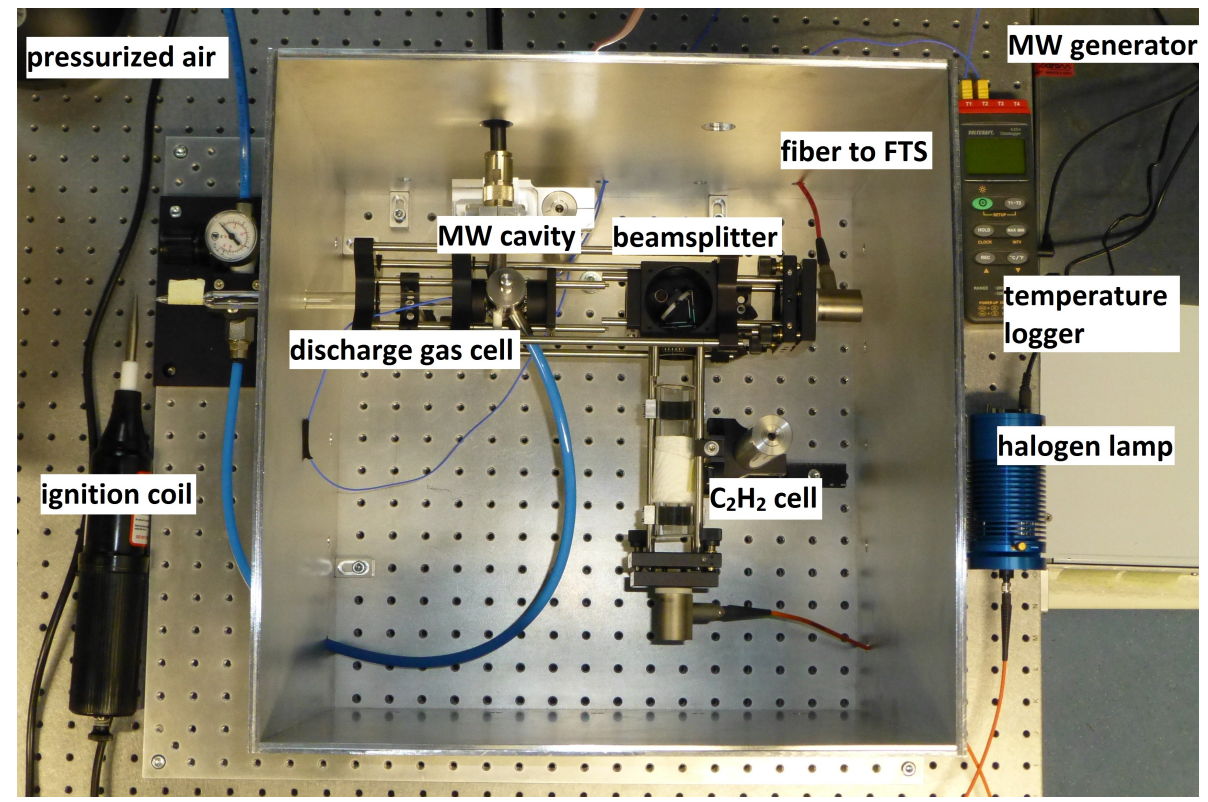

Figure 4.3: Picture of the microwave-induced discharge lamp located in the optics laboratory of the Institut für Astrophysik in Göttingen. Previously shown in Boesch et al. (2014).

The setup consists of the components needed to create the MIP, and of additional optics, gas cells, and lamps to carry out the experiments. Figures 4.3 and 4.4 show a picture and a schematic drawing of the complete experimental setup. The experiment is located inside an aluminum box with a ground space of $42 \times 42 \mathrm{~cm}$ and a height of $36 \mathrm{~cm}$. It is mounted on a portable aluminum breadboard $(50 \times 60 \mathrm{~cm})$ with a grid of M6 screw threads separated by $25 \mathrm{~mm}$. The breadboard has four rubber pads that ensure a firm stand. The aluminum enclosure serves as a Faraday cage to prevent leakage of MW radiation. It also absorbs noise produced by the flow of pressurized air which is needed for cooling inside the MW cavity. The microwave generator has dimensions of $48 \times 56 \times 14 \mathrm{~cm}$ and is located next to the aluminum box. The main components of the experimental setup are

- the MW generator,

- the MW cavity,

- the sealed discharge gas cell,

- a high-frequency coil for igniting the discharge,

- the acetylene $\left(\mathrm{C}_{2} \mathrm{H}_{2}\right)$ absorption gas cell for simultaneous wavelength reference,

- a halogen lamp for illuminating the absorption cell and for flat-field spectra.

The first four items belong to the actual discharge lamp, while the last two items are 


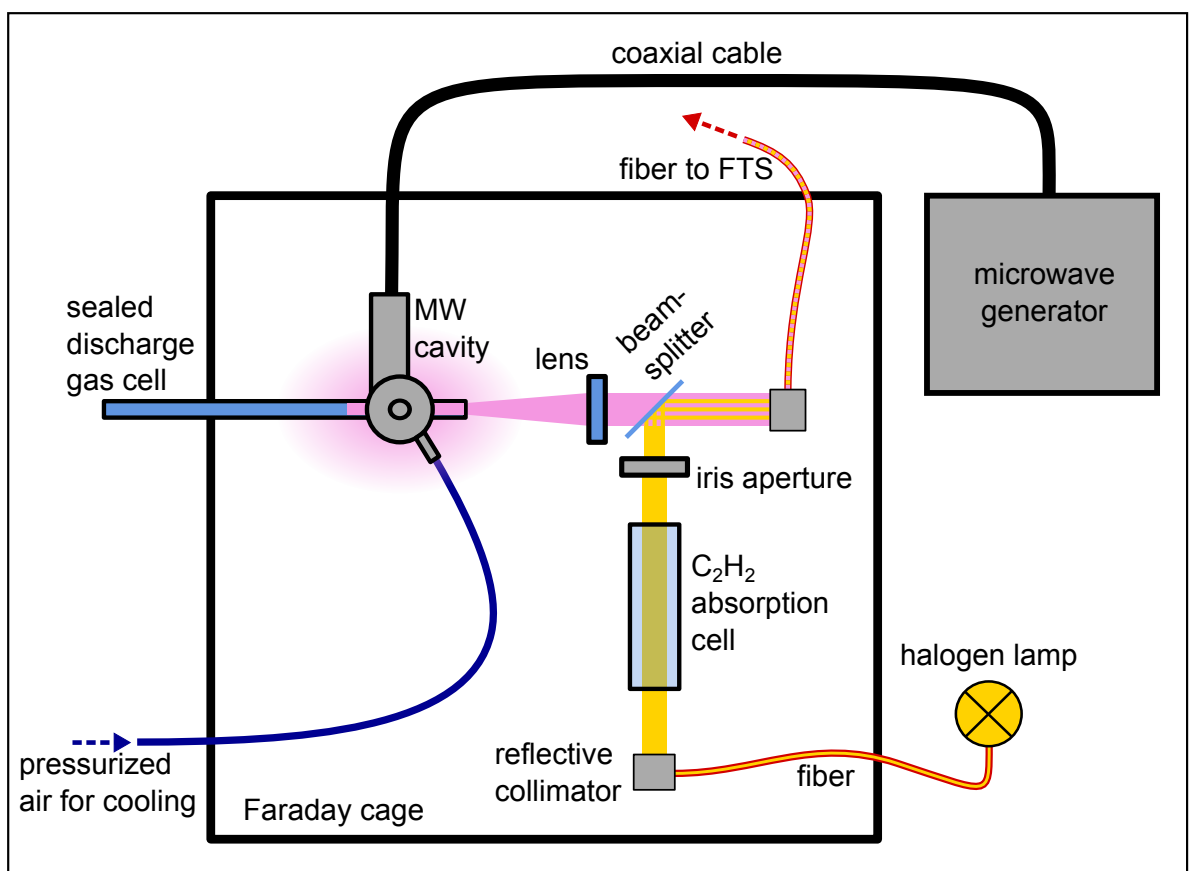

Figure 4.4: Schematic drawing of the experimental setup. A previous version of this drawing is shown in Boesch et al. (2014).

needed for calibration measurements. Not all components are needed for every type of measurement. Figure 4.4 shows the typical setup for simultaneous measurements of the discharge and the $\mathrm{C}_{2} \mathrm{H}_{2}$ absorption cell. The absorption cell serves as a relative wavelength reference to track drifts of the wavelength scale of the Fourier transform spectrometer (FTS) that is used to record spectra. The light from the halogen lamp illuminates the absorption cell and is combined with the light from the discharge at the beamsplitter (see Sects. 2.4.2 and 5.3 for more details on the simultaneous measurements). For observation of the sole discharge spectrum, the beamsplitter can be removed to obtain a higher signal. An optical fiber is used to feed the light from the experimental setup to the FTS. A main design characteristic of the constructed experiment is the usage of a sealed glass cell containing the gas for the discharge. It allows for a compact setup and little requirements on infrastructure to operate the lamp. Classically, an MIP is operated with a flowing gas system in a laboratory, i.e., there is a continuous gas flow from pressurized gas cylinders to a vacuum pump. However, a flowing gas system occupies a relatively large space and requires special appliances for the gas cylinders. Both are usually not available or desirable at an astronomical observatory.

Radiation security is an important aspect and a MW meter (IFP 05 C from Sairem) is available to check MW leakage. Measured values inside the aluminum enclosure are below $4 \mathrm{~mW} / \mathrm{cm}^{2}$ for input power of $50 \mathrm{~W}$ and no radiation is detected outside the Faraday cage. For household microwave ovens, which usually operate at powers of 500-1200 W, the maximum allowed leakage is $5 \mathrm{~mW} / \mathrm{cm}^{2}$ at a distance of $5 \mathrm{~cm}$ from the device (DIN EN 60335-2-25). 


\subsubsection{Microwave generator}

The input power for the lamp is provided by a microwave generator from Sairem. This generator is based on semiconductor technology which gradually replaces the older magnetrons. Magnetrons are based on the interaction of an electron beam with an electromagnetic field inside a glass or metal vacuum enclosure. They provided the first practical sources of high-frequency power, and were the only sources available for several decades. Today, semiconductor (solid-state) technology is used in the majority of microwave applications, especially at lower powers and lower frequencies as shown in the left part of Fig. 4.5 (Pozar, 2012, Sect. 11.5). Figure 4.5 also shows a picture of the microwave generator used for the experiments.

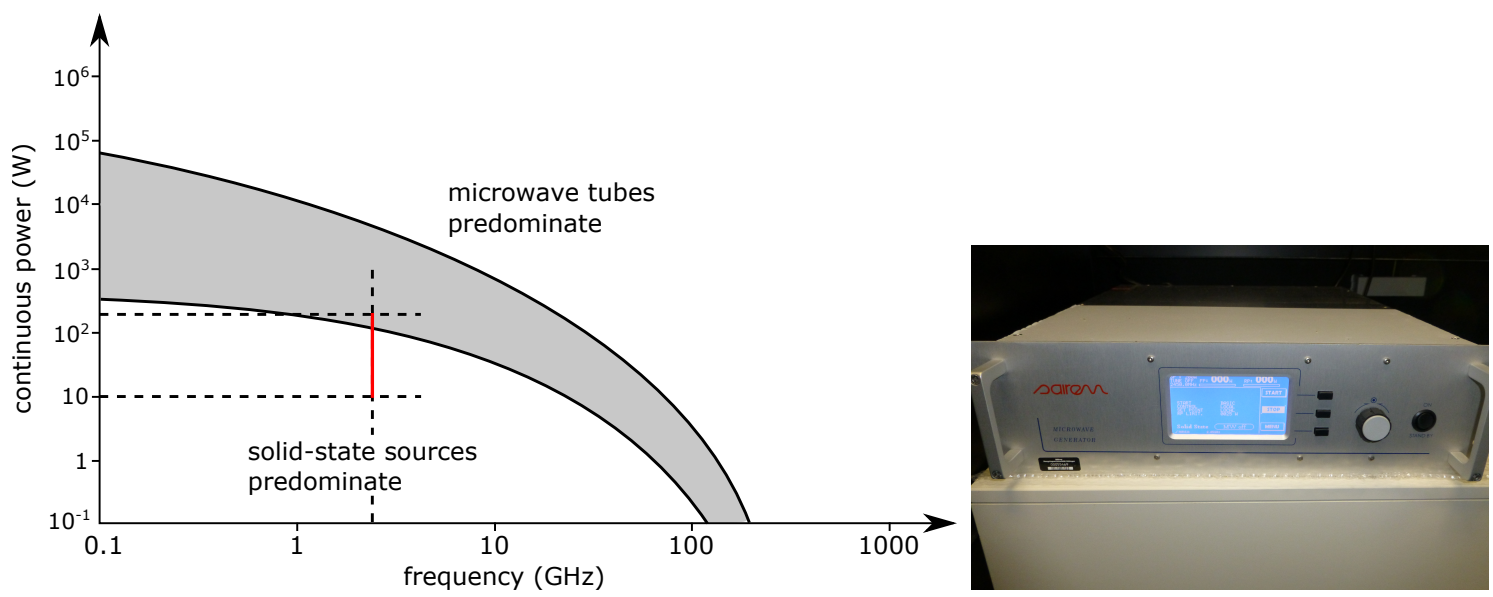

Figure 4.5: The graph illustrates the domains of the two types of $M W$ generators: magnetron vs. solid-state; adopted from Pozar (2012, Fig. 11.28). The red line marks the typical operational conditions in the experiment with a power range of 10-200 W at a frequency of 2.45 GHz. Picture on the right: Microwave generator used in the experimental setup.

The main advantages of the solid-state generator are according to the manufacturer: (i) a narrow and stable frequency spectrum even at low power; (ii) a detector with linear measurement of reflected and forward power; (iii) no high voltage inside the generator; and (iv) the possibility to adjust the frequency. Table 4.1 summarizes the specifications of the MW generator. The generator can be monitored and controlled externally through an RS232 connection. A Python script has been written for this purpose and is available on a RaspberryPi (miniature single-board computer) which can be accessed through the Institute's computer network. The microwave output is guided in a coaxial cable to the resonant cavity that is described in the next section.

\subsubsection{Microwave cavity}

The microwave cavity is a metal coaxial resonator. It has the physical dimensions to support standing waves and is a structure for focusing the microwave energy inside the discharge tube (Jankowski \& Reszke, 2011, Sect. 2.1). Two MW resonators were purchased 


\begin{tabular}{|c|c|}
\hline Manufacturer: & Sairem \\
\hline Model: & GMS200WSM56MPFCFST1IRVFAIT \\
\hline Frequency: & $\begin{array}{l}\text { central frequency at } 2450 \mathrm{MHz} \\
\text { adjustable between } 2430 \mathrm{MHz} \text { and } 2470 \mathrm{MHz} \text {, stepsize } 1 \mathrm{MHz}\end{array}$ \\
\hline Output power: & $0 \mathrm{~W}$ to $200 \mathrm{~W}$, stepsize $1 \mathrm{~W}$ \\
\hline Power stability: & $1 \%$ stability from $10 \%$ to $100 \%$ maximum power \\
\hline Ripple rate: & $<1 \%$ RMS from $10 \%$ to $100 \%$ \\
\hline Power rise time: & $<20 \mathrm{~ms}$ from $10 \%$ to $100 \%$ \\
\hline Microwave output: & coaxial cable with $\mathrm{N}$-type connector, $50 \Omega$ impedance \\
\hline
\end{tabular}

Table 4.1: Specifications of the solid-state microwave generator used for the MIP lamp.

from Opthos Instruments: a Broida-type (3/4-wave) cavity and an Evenson-type (1/4-wave) cavity. These two cavities operate at a MW frequency of $2.45 \mathrm{GHz}$ which corresponds to a wavelength of about $12 \mathrm{~cm}$. The Broida-type resonator has an effective length of about $9 \mathrm{~cm}$. It is also called a cylindrical cavity and can be seen in Fig. 4.1. The Evenson-type resonator has an effective length of about $3 \mathrm{~cm}$ and is shown in the left picture of Fig. 4.6. Similar cavities are discussed, among others, in the work by Fehsenfeld et al. (1965) and a modified design for the Evenson-type cavity is described by Regan \& Haugsjaa (1977).

The Broida-type cavity was used for the first realization of the experimental setup. According to the manufacturer, it provides a more uniform discharge compared to the Evenson-type cavity. However, I made the experience that the energy transmission from the microwaves into the discharge is not optimal, as indicated by the amount of reflected power. The reflected power, as measured at the MW generator, is the power that is not absorbed by the gas discharge but instead gets reflected back to the generator. In the experiments with the Broida-type cavity, the reflected power exceeded $30 \%$ of the forward power. According to the manual of the MW generator, the reflected power should be kept below $30 \%$ of the forward power to prevent damage to the instrument.

High reflected power results from a mismatch in resonant frequency or impedance between the cavity and the power supply (Fehsenfeld et al., 1965). In contrast to the Broida-cavity, the Evenson-cavity provides two tuning devices to match the impedance of the load to that of the generator. Referring to the left picture of Fig. 4.6, the coaxial cable from the MW generator connects to the stub on the left side of the cavity. The resonant frequency of the cavity is adjusted by means of the tuning stub (at the top) and the coupling is optimized with the slider on the right side which adjust the length of the inner conductor. With the two adjustment sticks, the reflected power can be minimized down to $0 \mathrm{~W}$.

\subsubsection{Gas cells}

I show different gas cells from the experiment in the right picture of Fig. 4.6. The upper three cells are used with the MIP lamp. They have an outer diameter of $12 \mathrm{~mm}$ to fit through the MW cavity and a length of about $20-25 \mathrm{~cm}$. From top to bottom, the three cells (1-3) represent an evolution in the gas cell design for this project. They are filled 
with gases at a reduced pressure of a few millibar.
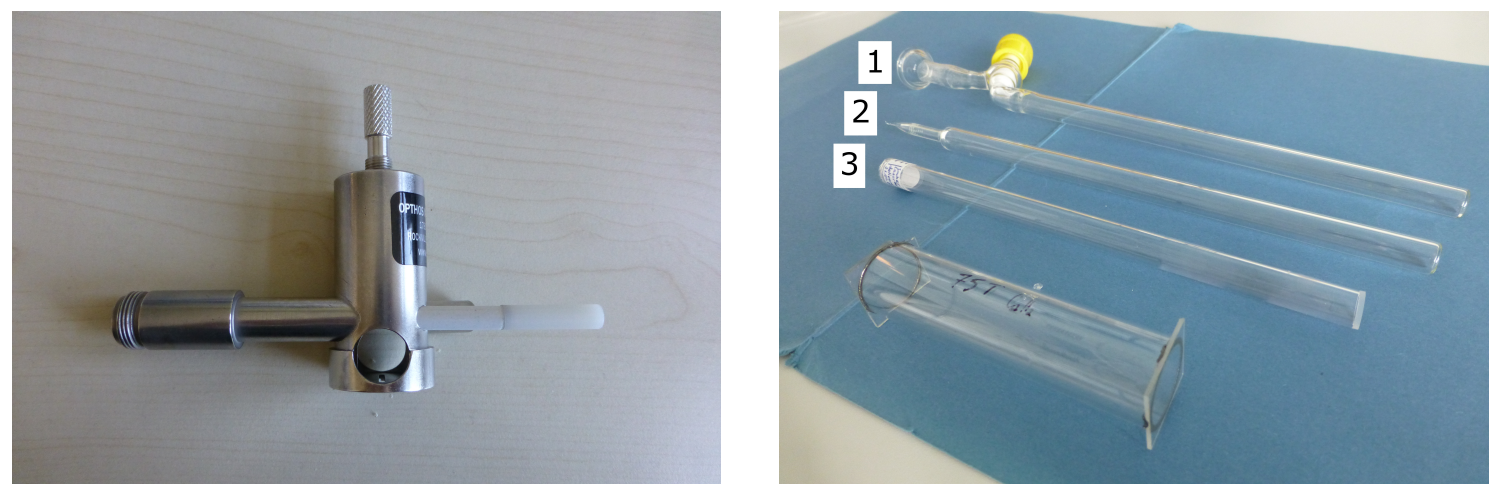

Figure 4.6: Left: Evenson-type $M W$ cavity. The hole at the center has a diameter of $13 \mathrm{~mm}$ to accommodate the gas cell. Right (gas cells from top to bottom): Discharge cell made from Pyrex with a valve; sealed discharge cell made from Pyrex; sealed discharge cell made from quartz with tilted quartz window; absorption cell with tilted windows and filled with $\mathrm{C}_{2} \mathrm{H}_{2}$.

Gas cell "1" was produced by the glass blower at the Chemistry Faculty in Göttingen. It is made from Pyrex and has a Young valve. The socket joint at the end allows to connect the cell to a special vacuum line in one of the laboratories at the Institut für Physikalische Chemie. This vacuum line was used to fill the cell. The cell with a valve could be reused to test different gases and pressures.

Gas cell "2" is also made from Pyrex but was produced by Glasgerätebau Ochs in Lenglern near Göttingen. This cell has a thin pipe at its end where the glass is easily melted with a torch. Thereby, the cell can be fire-sealed right after the filling process and leakage through a valve is prevented. The cell cannot be refilled after it has been sealed. The spectra recorded with gas cells " 1 " and " 2 " showed contamination from unwanted gases, e.g., residual air. It became clear that the available facilities for cell preparation and filling are not sophisticated enough to produce gas cells free of contamination.

Gas cell "3" was then purchased from Sacher Lasertechnik and is made from quartz glass. A plane quartz window is melted onto the front end of the cell with a small tilt. According to the manufacturer, the gas cell is baked at $10^{-8}$ Torr for 24 hours to remove impurities and then cooled to room temperature prior to filling the cell. Two cells filled with different gases were ordered: the " $\mathrm{N}_{2}$ cell" is filled with 2 mbar of high-purity dry nitrogen gas and the "CN cell" is filled with nitrogen $(2 \mathrm{mbar})$, carbon monoxide $(0.7 \mathrm{mbar})$ and helium (0.7 mbar). The latter gas cell produces emission from CN molecules, when the discharge is active. The gas mixture of the $\mathrm{CN}$ cell was determined during experiments at the Department of Chemistry and Biochemistry of the Old Dominion University in Norfolk ${ }^{1}$ : Using a flowing gas system, the partial pressures of the gas mixture could be varied while the MIP was active. Simultaneous observation of the MIP with an FTS allowed to select the partial pressures that maximize the vibrational bands of $\mathrm{CN}$ in the spectrum. Possible

\footnotetext{
${ }^{1}$ I thank Peter Bernath and Michael Dulick for the opportunity to visit their research group and for the support with the measurements.
} 
formation and excitation mechanism of $\mathrm{CN}$ in discharge plasmas have been discussed in the literature, but "[...] up to now the plasma chemistry of CN radicals and processes leading to the excitation of CN electronic states are still poorly understood" (Grigorian \& Cenian, 2011, p. 337). The $\mathrm{N}_{2}$ cell and the $\mathrm{CN}$ cell were used to record spectra for the analysis presented in Chap. 5 .

The gas cell at the bottom in Fig. 4.6 is filled with 100 mbar of acetylene $\left(\mathrm{C}_{2} \mathrm{H}_{2}\right)$ and is used in absorption as a relative wavelength reference. The cell has a diameter of $25 \mathrm{~mm}$ and a length of $10 \mathrm{~cm}$. Wedged windows are placed on both ends and are tilted at a small angle in opposite directions to avoid fringing effects. The $\mathrm{C}_{2} \mathrm{H}_{2}$ cell was purchased from Wavelength References, Inc.

\subsubsection{Further equipment}

Halogen lamp The high-power version of a halogen lamp from Ocean Optics (HL-2000HP-FHSA) is used for two purposes: first, to illuminate the $\mathrm{C}_{2} \mathrm{H}_{2}$ absorption cell as shown in Fig. 4.4 and, second, to record flat-field spectra. The lamp emits light in the wavelength range $360-2400 \mathrm{~nm}$ and has a nominal bulb temperature of $3000 \mathrm{~K}$. The temperature corresponds to a peak of the theoretical black-body curve at $969 \mathrm{~nm}$. The manufacturer provided calibrated spectral data from the same type of lamp operated at a lower voltage (HL-2000-FHSA) for wavenumbers above $6250 \mathrm{~cm}^{-1}$ (up to $1.6 \mu \mathrm{m}$ ). The maximum deviation of the measured spectral output from the theoretical black-body curve with the specified temperature is about $7 \%$. Based on this data, it is assumed that the high-power version of the lamp follows its corresponding black-body curve with a similar accuracy.

High-frequency coil The gas discharge is ignited with the high-frequency generator PG 100 Basic in combination with a hand-held probe from GNT.

Temperature logger The Voltcraft K204 Datalogger can record data from up to four K-type thermocouples. The sensors are distributed around the experiment. One sensor is usually installed at the position where the discharge gas cell enters the MW cavity to monitor the temperature inside the resonator.

Optics and fibers Table 4.2 lists optical components that are integrated into the setup. All parts are from Thorlabs.

\subsection{How to operate the discharge lamp}

The following list summarizes the steps to start the MIP lamp. I refer to the manual of the MW generator for more information on its different settings. 


\begin{tabular}{|c|c|c|}
\hline type & model & description \\
\hline \multirow[t]{2}{*}{ optical fibers } & FG550LEC & core diameter: $550 \mu \mathrm{m}$, length: $10 \mathrm{~m}$ (to FTS) \\
\hline & M38L01 & $\begin{array}{l}\text { core diameter: } 200 \mu \mathrm{m} \text {, length: } 1 \mathrm{~m} \text { (from halogen lamp } \\
\text { to absorption cell) }\end{array}$ \\
\hline collimator & RC08SMA-P01 & protected silver reflective collimator, $0.45-20 \mu \mathrm{m}$ \\
\hline beamsplitter & BSW23 & 50:50, infrared fused silica, coating: $0.9-2.6 \mu \mathrm{m}$ \\
\hline lens & LA 1255 & N-BK7, plano-convex, uncoated \\
\hline iris & SM1D12C & graduated ring-actuated iris diaphragm \\
\hline
\end{tabular}

Table 4.2: Optical components used in the experiment.

1. Insert discharge gas cell into the MW cavity. Confirm that it is secured in place and cannot move.

2. Turn on the cooling system by opening the valve for the pressurized air.

3. Turn on MW generator and set forward power: press the front panel knob once and turn the knob to adjust the power value.

4. Use the menu to set the maximum reflected power value. It should not be higher than $30 \%$ of the forward power during normal operation. Select the desired mode for the case the reflected power is reached: "RP Regul." regulates the forward power to keep the reflected power below the set value; "STOP on PR" shuts down the microwave generation automatically when the limit for the reflected power is reached.

5. Use the menu to set the start mode. The "RAMP" mode (e.g., with a delay of 20 seconds) allows to ignite the discharge at a low MW power before the limit for reflected power is reached. This is a helpful mode, because as long as the discharge is not ignited, all microwave power will be reflected back to the generator and, if set, can trigger the automatic shutdown.

6. Turn on the high-frequency generator and set the power by turning the knob. A value of $50 \%$ used to be sufficient to start the discharge.

7. Double-check if air-cooling is turned on. Press "START" button on front panel of MW generator to start microwave output.

8. Point the tip of the high-frequency coil close to the discharge cell and push trigger button on hand-piece. The discharge ignites and is sustained by the microwaves.

9. Check reflected power at MW generator. Reflected power must not be kept above $50 \mathrm{~W}$ for more than 1 minute and must be below $30 \%$ of forward power. Alternately, adjust the two tuning rods at the MW cavity to minimize the reflected power (eventually decrease forward power while operating at the cavity).

10. The forward power can be adjusted while the discharge is active (as described in step 3). Press "STOP" to turn off MW generation and stop the discharge. 


\section{Near-infrared wavelength calibration with molecular discharge spectra}

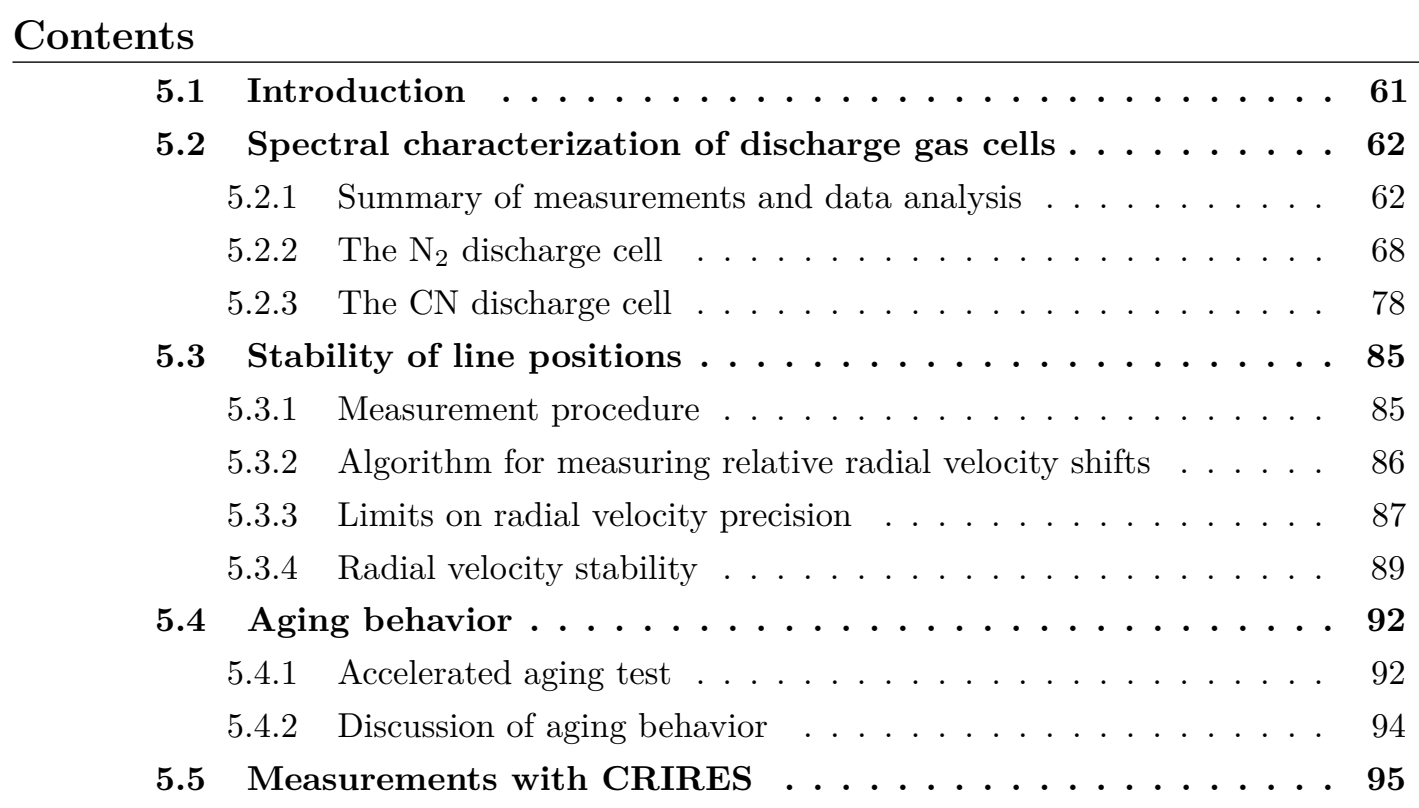

\subsection{Introduction}

Molecular discharge spectra recorded from a microwave-induced plasma (MIP) exhibit a large number of spectral lines in the near-infrared. I analyze spectral properties of the MIP lamp to evaluate its potential as a wavelength calibration source for astrophysical spectrographs in the wavelength range between approximately $1 \mu \mathrm{m}$ and $2 \mu \mathrm{m}$. The spectra of two different discharge gas cells are investigated. I use the term " $\mathrm{N}_{2}$ (discharge) cell" for the cell filled with 2 mbar of pure nitrogen. The second cell, the "CN (discharge) cell", is filled with nitrogen $(2 \mathrm{mbar})$, carbon monoxide $(0.7 \mathrm{mbar})$ and helium $(0.7 \mathrm{mbar})$. The latter gas cell produces emission from $\mathrm{CN}$ molecules when the discharge is active. See Sect. 4.3.4 for more details on the gas cells.

The analysis of the two discharge cells focuses on three aspects that are important in the context of wavelength calibration. First, I record high-resolution spectra for characterization. The characterization includes investigation of wavelength distribution and relative intensities of the spectral lines. A sufficient number of spectral lines is the most basic requirement for 
a spectrum to be usable for wavelength calibration. The distribution of spectral lines over a large wavelength range is especially important in high-resolution spectroscopy, where the recorded spectral range can be limited by detector size or can be spread over many echelle orders. The study of relative intensities is important because detectors are only sensitive to a limited dynamic range. Second, I investigate the wavelength stability of the spectra. A stable reference spectrum is needed to compare observations taken at different times, e.g., radial velocity (RV) measurements. Third, I explore the aging behavior of the MIP lamp, because wavelength calibrators need to have a constant spectral output over the anticipated time of operation. However, spectra of emission sources usually change over time and the lamps have a limited lifetime. For example, hollow cathode lamps (HCLs) experience aging related to sputtering processes and gas absorption (e.g., Kerber et al., 2007).

It is helpful to know the evolution of the project for a better understanding of the order of the presented measurements and results. As described in Sect. 1.3, the emission spectrum of the $\mathrm{CN}$ molecule was originally in the focus as a potential wavelength calibration source. $\mathrm{CN}$ molecules produce easily under conditions that provide donors of nitrogen and carbon (Ram et al., 2010). It turned out that the MIP spectra with emission lines of CN also exhibit lines from molecular nitrogen. In fact, the spectral lines from molecular nitrogen outnumber the $\mathrm{CN}$ lines, as we will see later in this chapter. In the course of the project it became clear that it is necessary to find out which spectral lines belong to molecular nitrogen and which lines originate from $\mathrm{CN}$ molecules. This assignment can be done with spectral line lists from the literature for each species. Spectral line lists for CN have been compiled by Ram et al. (2010) and Brooke et al. (2014), and can be readily used. For molecular nitrogen however, I could not find a line list that covers several thousands of wavenumbers in the near-infrared, although the spectrum of molecular nitrogen had been studied extensively in the past (see Lofthus \& Krupenie (1977) for an extensive review on the spectrum of molecular nitrogen). Therefore, I decided to first study a discharge of pure nitrogen gas using the $\mathrm{N}_{2}$ cell and to compile a line list for molecular nitrogen. Using this line list, I was then able to identify molecular nitrogen lines in the spectrum of the CN cell.

In Sect. 5.2, the spectral characterization of the two discharge cells is presented. I start with a summary of the measurements and the data analysis, before I separately present the results for the $\mathrm{N}_{2}$ cell and the CN cell. The wavelength stability was measured for spectra of both gas cells over 24 hours (Sect. 5.3). In Sect. 5.4, I show the results from a dedicated observation run to study the aging behavior of the MIP lamp. Observations of the MIP lamp with the CRIRES spectrograph are reported in Sect. 5.5.

\subsection{Spectral characterization of discharge gas cells}

\subsubsection{Summary of measurements and data analysis}

For the characterization of the discharge spectra, I used the experimental setup that is shown in Fig. 5.1. It is part of the complete setup that has been described in detail in Chap. 4. Because the $\mathrm{C}_{2} \mathrm{H}_{2}$ cell is not needed for these measurements, I removed the 
beamsplitter from the setup to increase the throughput and to reduce the influence of optical components on the recorded signal.

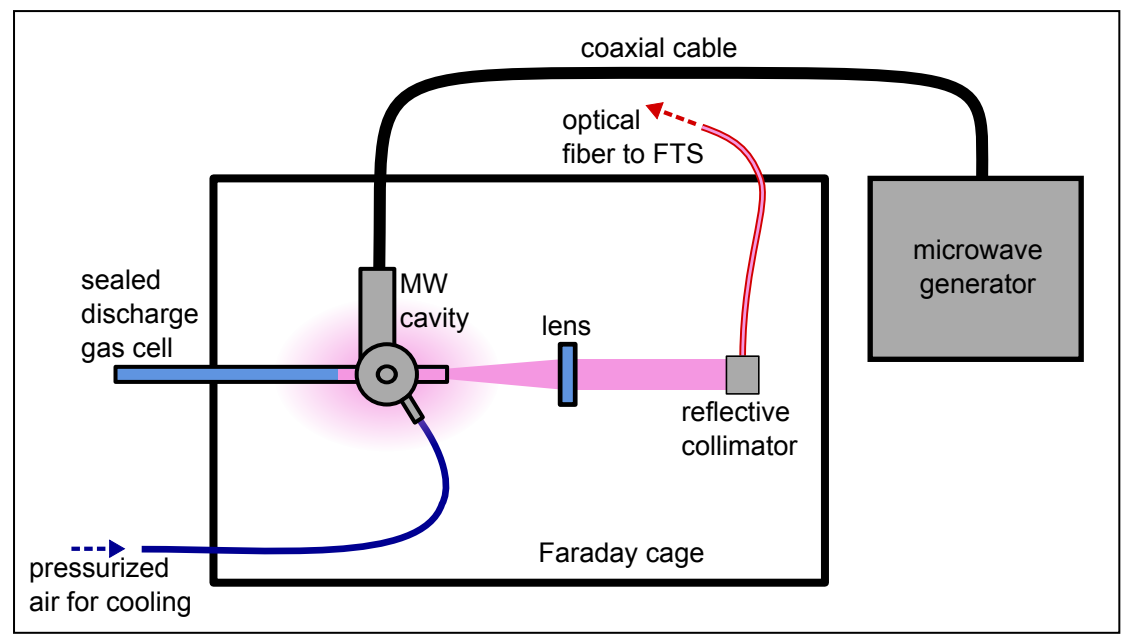

Figure 5.1: The experimental setup for characterizing the discharge gas cell. (Boesch $\& 3$ Reiners, 2015, Fig. 1)

On 14 February 2015, I recorded three high resolution spectra $\left((\Delta \tilde{\nu})_{\text {OPUS }}=0.018 \mathrm{~cm}^{-1}\right.$; spectral resolving power of $R \approx 560000$ at $\lambda=1 \mu \mathrm{m}$ ) of the gas discharge with the $\mathrm{N}_{2}$ cell. For each spectrum, the discharge was operated at a different microwave (MW) power. The power was set at the generator to $25 \mathrm{~W}, 50 \mathrm{~W}$, or $100 \mathrm{~W}$ for the three measurements. Subsequently, on 15 February 2015, I recorded one spectrum of the CN discharge cell at the same resolution with the MW generator operated at $50 \mathrm{~W}$. The experimental procedure and the settings of the FTS were the same for all measurements. The chosen resolution allows to record a symmetric interferogram minimizing phase corrections errors. For the FTS, I used an entrance aperture of $1.3 \mathrm{~mm}$, the $\mathrm{CaF}_{2}$ beamsplitter, and the nitrogen-cooled $\mathrm{InSb}$ detector. The final interferogram for each measurement was an average of 300 scans with a total scanning time of about $6 \mathrm{~h} 45 \mathrm{~min}$. The Norton-Beer-medium apodization function was applied.

In addition, three spectra of a sealed discharge cell filled with 2 mbar of argon gas were recorded: one spectrum was recorded before the measurements of the $\mathrm{N}_{2}$ cell, one between the measurements of the $\mathrm{N}_{2}$ cell and the $\mathrm{CN}$ cell, and one after the measurement of the CN cell. The emission lines of argon are used to correct the spectra for an absolute wavenumber scale. For these measurements, the argon gas cell simply replaced the $\mathrm{N}_{2}$ cell or CN cell in the experimental setup. Furthermore, I recorded spectra of a laboratory halogen source for the purpose of flat-fielding. The three flat-field spectra were also recorded before, between and after the measurements of the $\mathrm{N}_{2}$ cell and the $\mathrm{CN}$ cell. For these measurements, the discharge gas cell was removed from the experimental setup and the halogen lamp was positioned behind the MW cavity. The light of the halogen lamp shines through the MW cavity and follows the same path to the FTS as the light from the discharge.

Figure 5.2 shows a raw spectra of the $\mathrm{N}_{2}$ cell, and Fig. 5.3 shows the raw spectrum of 
the CN cell. Intensities are given in arbitrary units (a.u.) as obtained from the Fourier transform. Strong vibrational bands of molecular nitrogen and CN are identified for orientation as described in the captions of the respective figures. The transmittance of the optical components in the experiment and the detector sensitivity set the limits of the recorded spectral range.

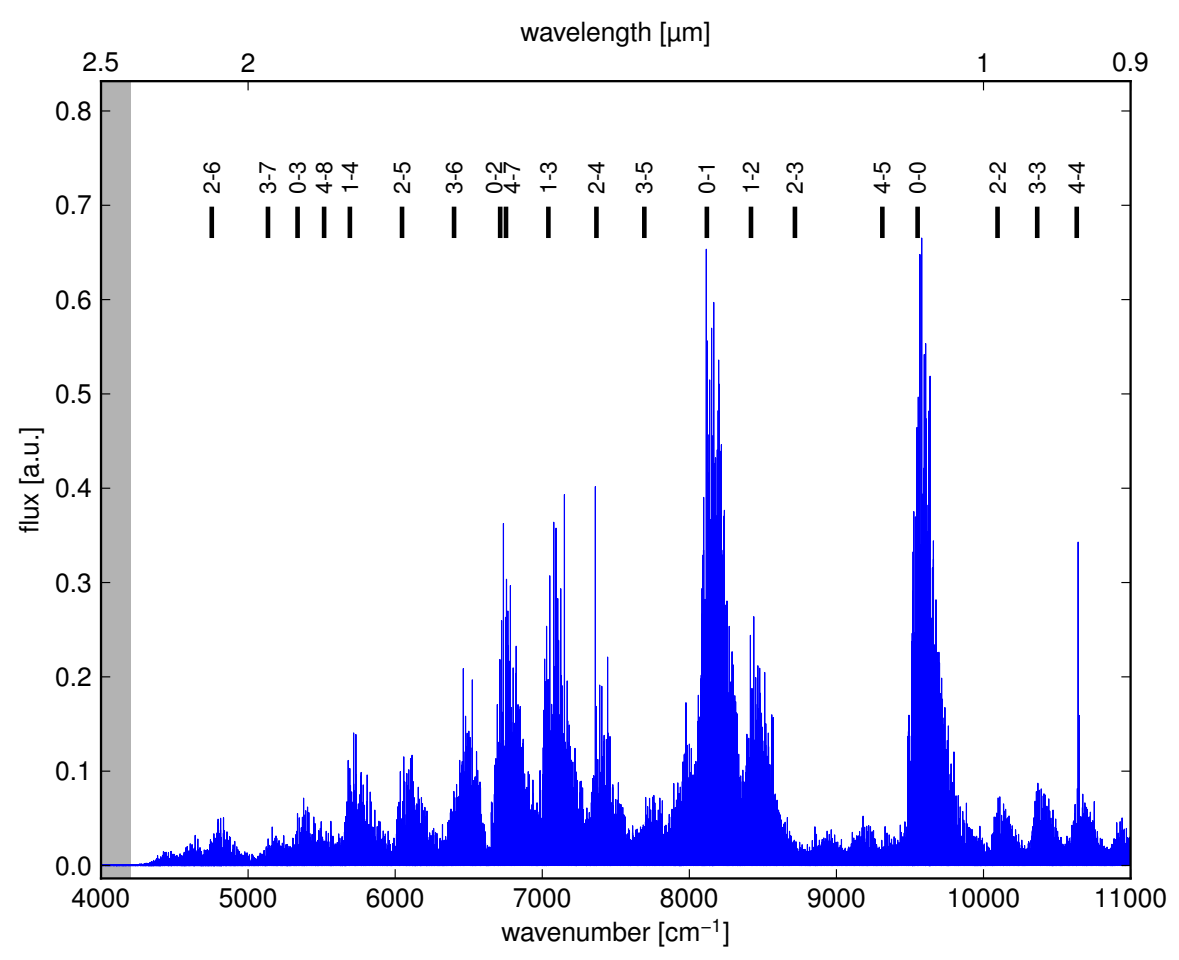

Figure 5.2: Raw emission spectrum of the nitrogen gas discharge operated at a $M W$ power of $50 \mathrm{~W}$. Strong vibrational bands from the First Positive System of ${ }^{14} N_{2}$ are labeled $\left(v^{\prime}-v^{\prime \prime}\right)$ for orientation using band origin wavenumbers as given by Gilmore et al. (1992). The spectral range below $4200 \mathrm{~cm}^{-1}$ (gray box) does not contain any emission lines and was used to determine the absolute noise level in the spectrum. (Boesch 8 Reiners, 2015, Fig. 2)

The data analysis for the characterization of the two discharge cells includes the following steps: determination of the absolute noise level; locating and fitting the emission lines with peak intensities above a minimum threshold value; correction for instrumental effects; and line identification. Most of these task have already been discussed in previous chapters. In the following, I give a summary of the analysis as carried out for the two discharge cells and give references to the respective sections in this thesis. The different steps of data analysis have also been outline in Boesch \& Reiners (2015, Sects. 3.1-3.5).

Noise level In each spectrum, the absolute noise level $\sigma_{\text {anl }}$ was determined as described in Sect. 3.4.1. The wavenumber range $4000-4200 \mathrm{~cm}^{-1}$ was used in the spectrum of the $\mathrm{N}_{2}$ cell (shaded region in Fig. 5.2), and the wavenumber range $4000-4050 \mathrm{~cm}^{-1}$ was used in the spectrum of the $\mathrm{CN}$ cell to calculate the absolute noise level. In addition, the baseline 


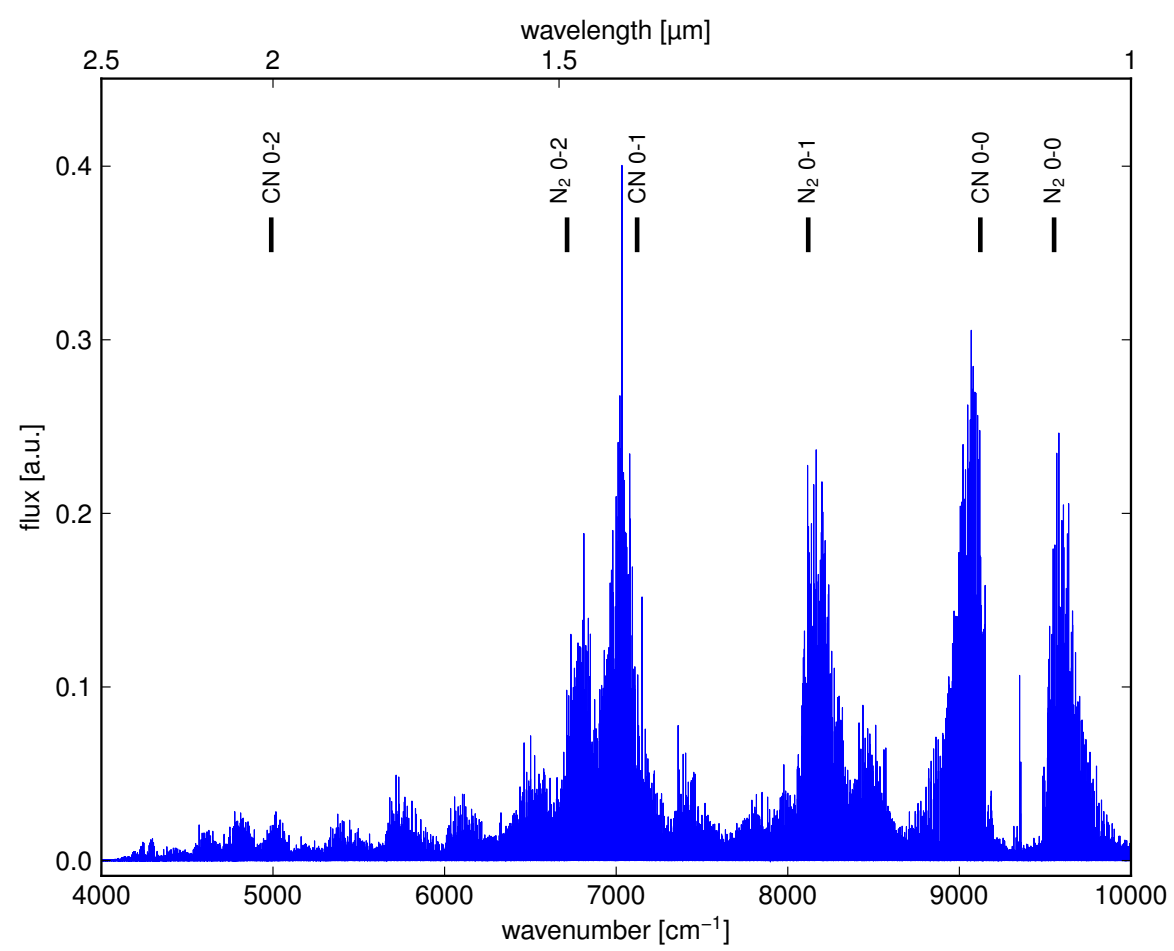

Figure 5.3: Raw emission spectrum of the CN cell. Strong vibrational bands from the First Positive System of ${ }^{14} \mathrm{~N}_{2}$ and the Red System of $C N$ are labeled with their vibrational quantum numbers for orientation using band origin wavenumbers from Gilmore et al. (1992) and Ram et al. (2010), respectively.

offset was determined and subtracted from each spectrum before further analysis. The calculated values for all spectra are listed in the first two rows of Table 5.1.

Peak finding and peak fitting In each spectrum, the parameters of the emission peaks were determined with the algorithm described in Sect. 3.4.2. Superpositions of Gaussian profiles were used to fit the emission lines. The minimum intensity of a peak to be included in the fitting process was $10 \sigma_{\mathrm{anl}}$. This value is a good compromise between including as many lines as possible in the analysis and obtaining good fit results even for low-intensity lines. The spectral lines in the wavenumber range $4500-11000 \mathrm{~cm}^{-1}$ were fitted for the $\mathrm{N}_{2}$ cell and the spectral lines in the wavenumber range $4500-10000 \mathrm{~cm}^{-1}$ were fitted for the CN cell. The third and fourth row in Table 5.1 list the minimum peak intensity and the total number of fitted lines for each spectrum.

Correction of line positions As described in Sect. 3.2.3, the wavenumber scale of the FTS is linearly shifted and the positions of the detected spectral lines need to be corrected using Eq. 3.9. Measured line positions of 22 spectral lines from the spectra of the argon discharge were compared with the corresponding Ar I line positions listed in the NIST database (Kramida et al., 2014, Norlén, 1973, Hirao et al., 2000). For each reference line, 


\begin{tabular}{l|l|ccc|c}
\hline \hline & & \multicolumn{3}{|c|}{$\mathrm{N}_{2}$ cell } & CN cell \\
& & $25 \mathrm{~W}$ & $50 \mathrm{~W}$ & $100 \mathrm{~W}$ & $50 \mathrm{~W}$ \\
\hline 1 & baseline offset [a.u.] & 0.00009 & 0.0001 & 0.0001 & 0.00009 \\
2 & absolute noise level [a.u.] & 0.0001 & 0.0001 & 0.0001 & 0.0001 \\
3 & minimum peak intensity [a.u.] & 0.001 & 0.001 & 0.001 & 0.001 \\
4 & number of peaks found and fitted & 40408 & 51776 & 58274 & 34982 \\
5 & wavenumber correction factor $\kappa_{\text {eff }}$ & \multicolumn{2}{|c|}{$(-0.85 \pm 0.85) \cdot 10^{-7}$} & $(-0.8 \pm 0.8) \cdot 10^{-7}$ \\
\hline
\end{tabular}

Table 5.1: Results from data reduction and analysis for the different recorded spectra.

an individual correction factor $\kappa=\left(\tilde{\nu}_{0, \text { ref }} / \tilde{\nu}_{0 \text {,measured }}\right)-1$ was calculated, where $\tilde{\nu}_{0, \text { ref }}$ is the argon line position from the NIST database and $\tilde{\nu}_{0 \text {,measured }}$ is the corresponding line position measured in the recorded spectrum. I show in the left panel of Fig. 5.4, the individual correction factors $\kappa$ as a function of the wavelength of the argon lines. The errorbars are calculated through error propagation of uncertainties in the line positions as listed in the original reference (a conservative value of $0.003 \mathrm{~cm}^{-1}$ was adopted; Norlén, 1973) and of uncertainties in the determination of the line positions in the recorded spectrum. For each calibration spectrum, an effective correction factor $\kappa_{\text {eff }}$ was calculated which is the weighted average of the 22 individual correction factors. The effective correction factors are $\kappa_{\text {eff, before }}=(-0.9 \pm 0.8) \cdot 10^{-7}, \kappa_{\text {eff, between }}=(-0.8 \pm 0.8) \cdot 10^{-7}$ and $\kappa_{\text {eff, after }}=(-0.8 \pm 0.8) \cdot 10^{-7}$ for the observations of the argon discharge taken before, between and after the measurements of the $\mathrm{N}_{2}$ cell and $\mathrm{CN}$ cell, respectively. The final correction values are based on $\kappa_{\text {eff, before }}$ and $\kappa_{\text {eff, between }}$ for the measurements of

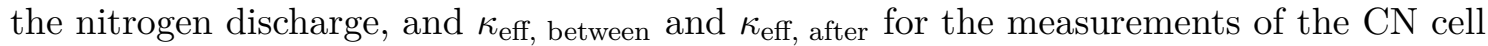
(conservative error propagation was applied). They are listed in the last row of Table 5.1.

Correction of line widths As described in Sect. 3.2, spectral features are broadened by the instrumental line shape (ILS) function of the FTS. The width of the ILS depends primarily on the scan length of the interferometer and the applied apodization. To calculate the natural line widths before instrumental broadening, I make the assumption that the spectral lines and the ILS function have the shape of Gaussian profiles. This is a reasonable approximation for the ILS profile produced by the applied Norton-Beermedium apodization function (compare Fig. 5.3 in Wartewig, 2003). The instrumental broadening can be described by a convolution of the spectral line with the ILS function. The convolution of two Gaussian functions results in another Gaussian function and the width of the convolved profile is equal to the squared sum of the original profiles' widths (compare, e.g., Woan, 2000). Therefore, we can calculate the corrected full width at half maximum of a spectral line, $F W H M_{\mathrm{c}}$, using $F W H M_{\mathrm{c}}=\sqrt{F W H M^{2}-F W H M_{\text {instr }}^{2}}$, where $F W H M$ is the uncorrected full width at half maximum of the spectral line, and $F W H M_{\text {instr }}$ is the full width at half maximum of the ILS function. The best estimate for $F W H M_{\text {instr }}$ is the resolution set in the OPUS software $(\Delta \tilde{\nu})$ OPUS multiplied by the correction factor for the applied apodization (see Table 3.1). This results in $F W H M_{\mathrm{instr}}=0.017 \mathrm{~cm}^{-1}$.

Measurements were carried out to estimate uncertainties for this correction. The recorded 

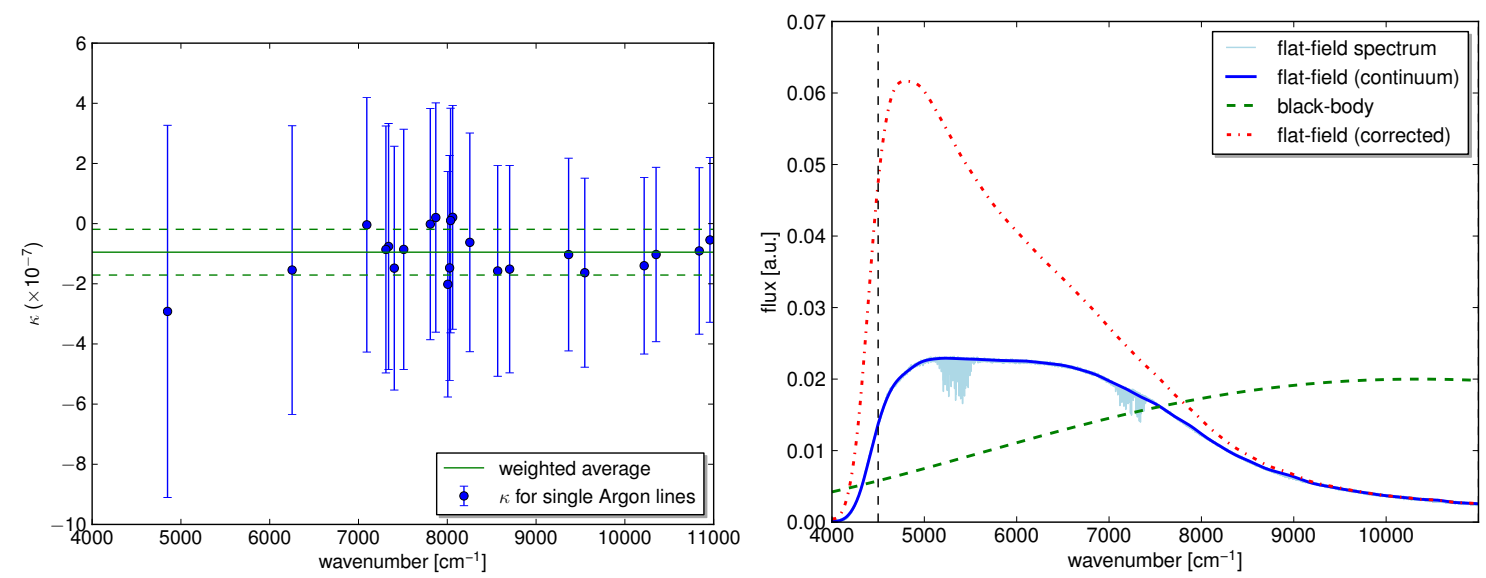

Figure 5.4: Left: Correction factor $\kappa$ for the absolute wavenumber scale of the FTS as determined for 22 atomic argon lines. The horizontal line indicates the weighted average (solid line) with its error margin (dashed lines). Shown are the data for the argon spectrum recorded before the measurements of the nitrogen spectra. Right: Spectrum of a halogen lamp is used as a flat-field (spectrum shown in light blue with absorption bands from atmospheric water). The dark blue line describes the continuum of this spectrum. The green dashed curve shows the theoretical normalized curve of a blackbody at the halogen lamp's bulb temperature of $3000 \mathrm{~K}$ (scaled by a factor of 1/50 for visualization). The red dot-dashed curve is the flat-field continuum divided by the theoretical normalized blackbody spectrum. The raw intensities of the spectral lines are corrected by the latter curve. The vertical dashed line indicates $4500 \mathrm{~cm}^{-1}$. (Boesch 83 Reiners, 2015, Figs. 4 and 5)

spectral line of a monochromatic light source has the finite line width of the ILS function. In the laboratory, the device which best resembles a monochromatic light source is a frequency-locked laser. On 4 March 2015, the spectrum of a Toptica DL 100 laser was recorded. The laser was locked to the D2 hyperfine transition of rubidium and has an expected line width of less than $100 \mathrm{kHz}(<0.1 \mathrm{~m} / \mathrm{s} \text {; compare Huke et al., 2015 })^{1}$. The position of the laser line is $\tilde{\nu}_{0} \approx 12820 \mathrm{~cm}^{-1}\left(\lambda_{0} \approx 780 \mathrm{~nm}\right)$. The measured width of the line was $F W H M_{\text {laser }}=0.0186 \mathrm{~cm}^{-1}$. This value is about $9 \%$ larger than the theoretical width $F W H M_{\text {instr }}$ caused by the finite scan length of the FTS in combination with the applied apodization function. The difference of the theoretical and the measured value could be caused by the finite entrance aperture or misalignment of the beams inside the FTS.

Correction of line intensities The relative intensities in the spectrum are affected by the wavelength dependent transmittance of the optical components and the detector sensitivity. Flat-field measurements of a laboratory halogen source were used to determine the intensity modulations and to correct the peak intensities. The halogen lamp (see Sect. 4.3.5) has a bulb temperature of $3000 \mathrm{~K}$ and its spectrum is shown in the right panel of Fig. 5.4. The recorded spectrum is shown in light blue recognizable by the two absorption bands from atmospheric water. The theoretical normalized spectrum of a black body with a

\footnotetext{
${ }^{1}$ I thank Philipp Huke for assisting with the measurements of the frequency-locked laser.
} 
temperature of $3000 \mathrm{~K}$ is given as the green dashed curve. It is clearly visible that the response of the measurement system is less sensitive towards larger wavenumbers. The continuum of the recorded black body spectrum (dark blue curve) is divided by the theoretical curve (green curve) to obtain the final flat-field signal (red dot-dashed curve). The intensity $I$ of a detected spectral line is divided by the latter curve at the corresponding wavenumber to obtain a flat-field corrected intensity $I_{\mathrm{c}}$. Based on information about the spectral output of the halogen lamp (see Sect. 4.3.5), the uncertainty in the flat-field process is assumed to be about $10 \%$.

\subsubsection{The $\mathrm{N}_{2}$ discharge cell ${ }^{2}$}

Three spectra were recorded of the $\mathrm{N}_{2}$ discharge cell with the MW generator operated at different MW powers of $25 \mathrm{~W}, 50 \mathrm{~W}$, and $100 \mathrm{~W}$. The analysis starts with an identification of atomic and molecular lines with data from the literature. For molecular nitrogen, only two line lists published by Effantin et al. (1979) and Ferguson et al. (1992) were found that covered at least some parts of the observed spectral range. In Sects. 5.2.2.4 and 5.2.2.5, I discuss in more detail the line intensities and line density for the discharge operated at $50 \mathrm{~W}$. I complete the spectral characterization of the $\mathrm{N}_{2}$ cell with a comparison of line widths and line intensities as a function of the three different MW powers. The results from this section have been published in Boesch \& Reiners (2015) together with spectral line lists of the nitrogen discharge.

\subsubsection{Atomic line identification}

All emission peaks in the spectrum were checked against line lists from the atomic line database from NIST (Kramida et al., 2014) for N I, N II, O I, and Ar I. We checked for oxygen and argon because they are the most abundant species in the atmosphere after nitrogen, and would be obvious contaminators, if the cell was not filled and sealed properly. We only used lines with observed wavelength values in the NIST database. For a match, we require the distance between the recorded line and the database to be less than the line's half width at half maximum $\left(\mathrm{HWHM}_{\mathrm{c}}=\mathrm{FWHM}_{\mathrm{c}} / 2\right)$. The probability that the position of a line from the literature matches a line in our recorded spectrum by chance is $20 \%, 30 \%$ and $38 \%$ for the measurements with MW power of $25 \mathrm{~W}, 50 \mathrm{~W}$ and $100 \mathrm{~W}$, respectively, because of the many densely-spaced spectral lines.

Although up to 35 oxygen lines (53\% of the lines listed in the NIST database) and 35 argon lines $(57 \%)$ fulfill the above mentioned $\mathrm{HWHM}_{\mathrm{c}}$ criterion, we judge them to be misidentified in the crowded spectrum. We come to this assessment, because the determined $\mathrm{FWHM}_{\mathrm{c}}$ of the lines do not follow the expected linear trend with wavenumber (see Sect. 5.2.2.6 and compare with Fig. 5.9) and the peak intensities $I_{\mathrm{c}}$ do not change by the same amount when the MW power is altered (compare with Fig. 5.10). The absence of lines from oxygen and argon indicates that no leakage of air into the gas cell had occurred.

\footnotetext{
${ }^{2}$ Sections 5.2.2.1, 5.2.2.2, 5.2.2.4, 5.2.2.5 and 5.2.2.6 have been published in Boesch \& Reiners (2015) as Sects. 3.6, 3.7, 4.2, 4.3 and 4.4 , respectively.
} 
For nitrogen, only lines from the neutral atom could be identified with confidence. This is not surprising because the degree of ionization is low in this kind of electrodeless discharge lamp (Thorne et al., 1999).

\subsubsection{Identification of molecular nitrogen lines}

We use the spectral line lists with branch designations for molecular nitrogen transitions in the wavenumber range of our spectra from Effantin et al. (1979) and Ferguson et al. (1992). As for the atomic line identification, we require that the distance to the literature value is less than the line's half width at half maximum.

The work by Effantin et al. (1979) provides line lists for the (0-0) band of the $\mathrm{B}^{3} \Pi_{\mathrm{g}}-\mathrm{A}^{3} \Sigma_{\mathrm{u}}^{+}$ system of ${ }^{14} \mathrm{~N}_{2}$ and ${ }^{15} \mathrm{~N}_{2}$. The lists consist of measured wavenumbers for 21 branches and calculated wavenumbers for 27 branches. The observed and calculated wavenumbers differ by less than $0.01 \mathrm{~cm}^{-1}$, where both values are given. Effantin et al. (1979) did not observe lines from the six branches designated $\mathrm{R}_{13}, \mathrm{Q}_{13}, \mathrm{P}_{13}, \mathrm{R}_{31}, \mathrm{Q}_{31}$, and $\mathrm{P}_{31}$. Our nitrogen gas contains the natural ratio of isotopes $\left({ }^{14} \mathrm{~N}_{2}:{ }^{15} \mathrm{~N}_{2}=99.636: 0.364\right.$; Lide 2006) and therefore only very faint lines of ${ }^{15} \mathrm{~N}_{2}$ are expected, if any at all. We do not include them in the analysis. For ${ }^{14} \mathrm{~N}_{2}$, we can identify up to $80 \%$ of the lines from the literature, including some from the above mentioned branches that were not observed by Effantin et al. (1979).

Furthermore, we identified up to $89 \%$ of the lines from four vibrational bands of the Meinel System of ${ }^{14} \mathrm{~N}_{2}^{+}$as listed in Ferguson et al. (1992). The four bands have their origins at $9016 \mathrm{~cm}^{-1}(0-0), 6841 \mathrm{~cm}^{-1}(0-1), 10889 \mathrm{~cm}^{-1}(1-0)$, and $6572 \mathrm{~cm}^{-1}(1-2)$.

The HITRAN2012 database (Rothman et al., 2013) lists additional 584 quadrupol transitions in the electronic ground state of ${ }^{14} \mathrm{~N}_{2}$ (in the wavenumber range $4500-11000 \mathrm{~cm}^{-1}$ ). The number of matches in line position $(17 \%, 26 \%$, and $34 \%$ for our three measurements with $\mathrm{MW}$ power of $25 \mathrm{~W}, 50 \mathrm{~W}$, and $100 \mathrm{~W}$, respectively) is below the probability for a random match for each spectrum, as stated in the previous section.

\subsubsection{Summary of detected spectral lines}

The number of spectral lines that have been detected in the three spectra are shown in Table 5.2. We observe that the total number of detected lines increases with higher MW power: the peak intensities of the spectral lines are larger for a higher MW power, while the noise level stays relatively constant (compare Table 5.1). Consequently, more lines exceed the minimum peak intensity threshold.

At the same time, the number of lines without a flag decreases. One or more flags can be assigned to a spectral line during the data analysis process. They mark noticeable problems that might effect the line's parameters. For completeness, the different causes for a flag are summarized. During the fitting process, flags are assigned if fit parameters do not converge within boundary conditions, or if line fits have large residuals (see also Sect. 3.4.2). A line is flagged as a blended line if a neighboring line is closer than the sum of the two lines' FWHM and if the neighboring line has at least half of the line's peak intensity. The 
second criterion considers the fact that a strong line influences the line shape of a weak neighboring line more than vice versa. Furthermore, a line with a determined FWHM that is narrower than the measurement's resolution is flagged and it could be an artifact in the spectrum. Finally, we consider that the widths of lines from the same species (same mass) should follow a similar linear trend as a function of wavenumber (see also Sect. 5.2.2.6): a spectral line with a $\mathrm{FHWM}_{\mathrm{c}}$ that deviates more than five times from the linear trend for its species is flagged and could, e.g., be an unresolved blend; for unidentified lines not assigned to a species, this criterion is modified and a flag is assigned if the error on the FHWM is five times larger than the average error on the FWHM of all lines.

\begin{tabular}{|c|c|c|c|}
\hline & $25 \mathrm{~W}$ & $50 \mathrm{~W}$ & $100 \mathrm{~W}$ \\
\hline \multirow{2}{*}{$\begin{array}{l}\text { Total number of lines } \\
\text { thereof lines without flags }\end{array}$} & 40408 & 51776 & 58274 \\
\hline & 24585 & 24863 & 22723 \\
\hline identified N I lines $^{a}$ & 25 & 27 & 29 \\
\hline thereof lines without flags & 17 & 14 & 17 \\
\hline${\text { identified } \mathrm{N}_{2} \text { lines }}^{b}$ & 860 & 953 & 1003 \\
\hline thereof lines without flags & 632 & 636 & 611 \\
\hline identified $\mathrm{N}_{2}^{+}$lines ${ }^{c}$ & 886 & 898 & 903 \\
\hline thereof lines without flags & 542 & 476 & 395 \\
\hline unidentified lines & 38637 & 49898 & 56339 \\
\hline thereof lines without flags & 23394 & 23737 & 21700 \\
\hline
\end{tabular}

Table 5.2: Number of spectral lines in the three spectra. Notes: ${ }^{(a)}$ Out of 29 lines from the NIST database. ${ }^{(b)}$ Out of 1260 lines of the $(0-0)$ band of the $B^{3} \Pi_{g}-A^{3} \Sigma_{u}^{+}$system of ${ }^{14} N_{2}$ from Effantin et al. (1979). ( ${ }^{(c)}$ Out of 1017 lines of the $(0-0),(0-1),(1-0)$, and $(1-2)$ bands of the $A^{2} \Pi_{u}-X^{2} \Sigma_{g}^{+}$system of ${ }^{14} N_{2}^{+}$from Ferguson et al. (1992). (Boesch 83 Reiners, 2015, Table 4)

About $90 \%$ of all flags are due to blends. For the three measurements conducted with $25 \mathrm{~W}, 50 \mathrm{~W}$, and $100 \mathrm{~W}$, the percentage of blended lines is $35 \%, 47 \%$, and $55 \%$, respectively. The increase of blended lines with higher MW power can be explained with the broader FWHM of the lines and by the overall larger number of lines. The broader FWHM and the larger number of detected lines makes it also more likely that lines are assigned to one of the species.

About 3-4\% of all detected lines could be assigned to atomic nitrogen (N I) or molecular nitrogen $\left(\mathrm{N}_{2}\right.$ or $\left.\mathrm{N}_{2}^{+}\right)$. The majority of lines remains unidentified. Theoretical treatment of the molecular transitions and spectral modeling are needed to confirm that these lines originate from nitrogen. We tried to fit the nitrogen spectrum with the program PGOPHER ${ }^{3}$ using spectroscopic constants given in the literature as initial values. Spectroscopic constants for molecular nitrogen are given, e.g., in Laher \& Gilmore (1991) and Roux et al. (1983). Unfortunately, the constants in these two papers could not be used to accurately reproduce the recorded spectra. Laher \& Gilmore (1991) only provide vibrational and rotational constants, but no further constants, e.g., for spin-orbit coupling. These constants are not sufficient to reproduce the complicated structure of the spectrum (see also Akatsuka,

\footnotetext{
${ }^{3}$ PGOPHER, a Program for Simulating Rotational, Vibrational and Electronic Structure, C. M. Western, University of Bristol, http://pgopher.chm.bris.ac.uk
} 
2012). Roux et al. (1983) do provide values for many constants for several vibrational levels of the First Positive System of $\mathrm{N}_{2}$, however, it appears that they used a different Hamiltonian for their calculations as the one implemented in the PGOPHER program ${ }^{4}$. The assignment of all lines in the nitrogen spectrum using theoretical modeling is desirable, but is not necessary for the following analysis of spectral properties. Because the cell is filled with nitrogen gas of high purity (see also Sect. 4.3.4), it is believed that the vast majority of spectral lines originates from molecular nitrogen. This assumption is supported by the observation that no significant amounts of oxygen and argon have been detected in the spectrum.

\subsubsection{Line intensities}

We present the distribution of line intensities using the spectrum of the discharge operated at MW power of $50 \mathrm{~W}$. Figure 5.5 displays the flat-field corrected line intensities $I_{\mathrm{c}}$ as a function of wavenumber. The corresponding raw spectrum is shown in Fig. 5.2. At lower intensities, the distribution exhibits an envelope with a shape related to the applied flat-field curve (compare with Fig. 5.4 [right panel]). This means that our measurements are less sensitive to fainter lines toward larger wavenumbers, owing to the response curve of the InSb detector. It can therefore be expected that all emission lines with a peak intensity $I_{\mathrm{c}} \geq 0.4$ a.u. are included in our spectra and line lists, but that the sample is incomplete for fainter lines.

A histogram of the peak intensities $I_{\mathrm{C}}$ is shown in Fig. 5.6, again exemplary for the discharge operated at $\mathrm{MW}$ power of $50 \mathrm{~W}$. The distribution peaks at around $0.1 \mathrm{a} . \mathrm{u}$. and decreases quickly toward smaller intensities ${ }^{5}$. The shape of the distribution at intensities below 0.4 is biased by the wavenumber-dependent detection efficiency. The total dynamic range of the spectral lines covers about four orders of magnitude. Figure 5.6 also highlights the fraction of lines with and without flags relative to the total number of detected lines as a function of intensity. For all three measurements, the percentage of flagged lines stays above $40 \%$ for the lower peak intensities between 0.01 and 0.1 , and decreases to a value below $25 \%$ for intensity values above 10 .

Near-infrared detectors used for astrophysical spectrometers can usually distinguish line intensities over a dynamic range of two orders of magnitude. Therefore, only a fraction of the spectral lines of the molecular nitrogen spectrum are usable for wavelength calibration of astrophysical spectrographs in practice. However, as the utilization of a spectral line of molecular nitrogen for wavelength calibration should not depend on its intensity, an observer is flexible in selecting the appropriate exposure time for a certain calibration frame.

\footnotetext{
${ }^{4}$ This issue was also mentioned by Brett McGuire in his Bachelor's Thesis "Design and Implementation of a Liquid Nitrogen-Cooled Hollow Cathode Discharge Source for the Study of the Reaction $\mathrm{H}_{3}^{+}+\mathrm{H}_{2} \rightarrow \mathrm{H}_{2}+\mathrm{H}_{3}^{+}$at Physically Relevant Temperatures and the Study of the $\mathrm{B}^{3} \Pi_{g} \rightarrow \mathrm{A}^{3} \Sigma_{u}^{+}$Band System of $\mathrm{N}_{2}$ ", University of Illinois (2009), and private communication.

${ }^{5}$ The designation for arbitrary units, a.u., for the intensity is dropped in the remainder of the article for better readability.
} 


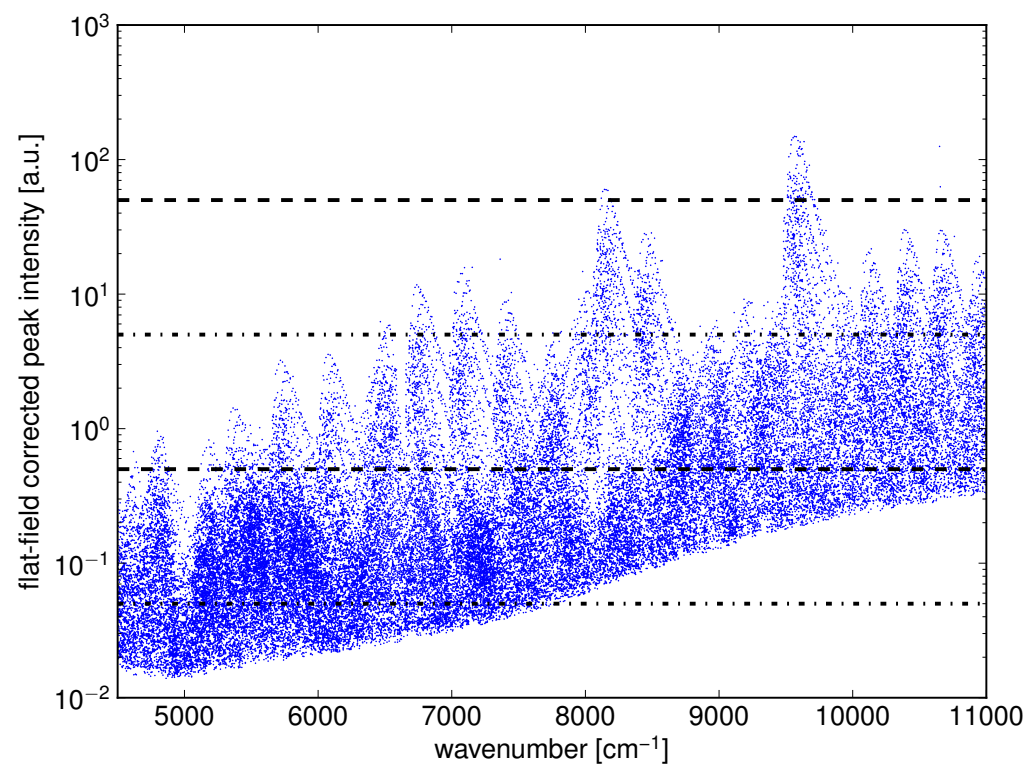

Figure 5.5: Line intensities after flat-field correction as a function of wavenumber. Note the logarithmic scale. The horizontal lines indicate intensity ranges used in the upper panel (dashed lines) and lower panel (dash-dotted lines) of Fig. 5.8. Data from discharge operated at MW power of $50 \mathrm{~W}$. (Boesch \& Reiners, 2015, Fig. 7)

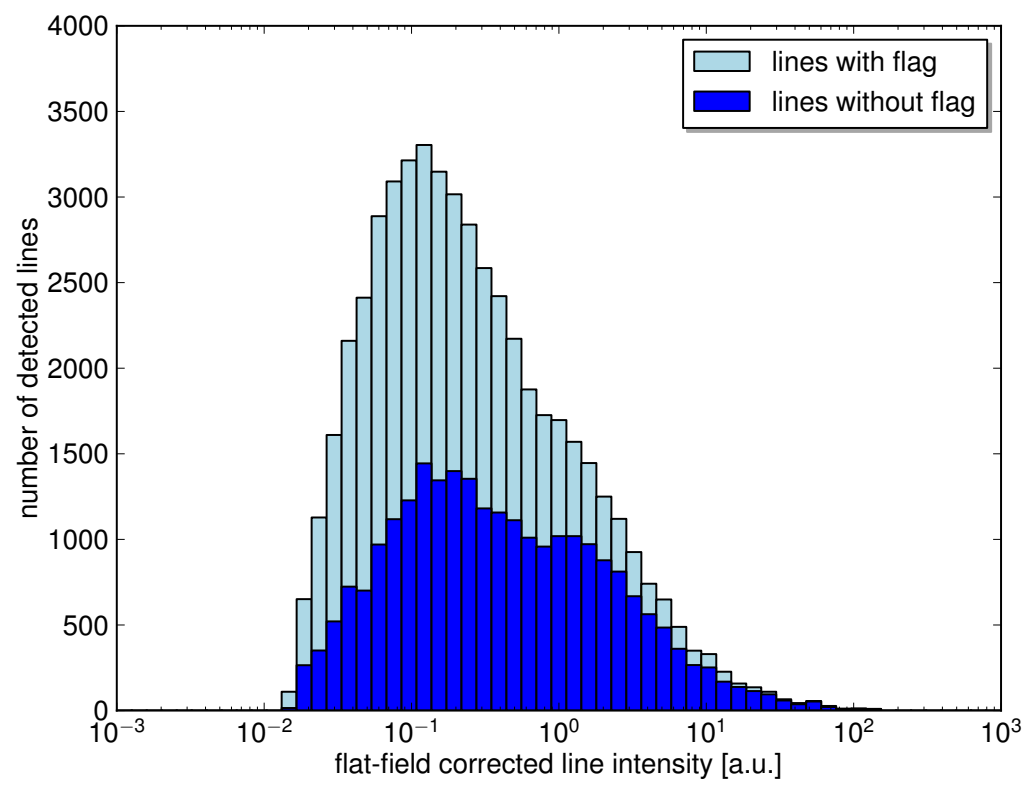

Figure 5.6: Histogram of the peak intensities $I_{c}$ of the detected lines. Bars are stacked on top of each other. Data from discharge operated at $M W$ power of $50 \mathrm{~W}$. (Boesch 6 Reiners, 2015, Fig. 8) 


\subsubsection{Line density}

The spectrum of the nitrogen gas discharge exhibits emission lines over the complete spectral range under investigation. A histogram of the line distribution is shown in Fig. 5.7 for all three measurements. Around 350 to 1300 lines are located within one bin covering $100 \mathrm{~cm}^{-1}$. The number of lines in each bin increases with higher operating MW power.

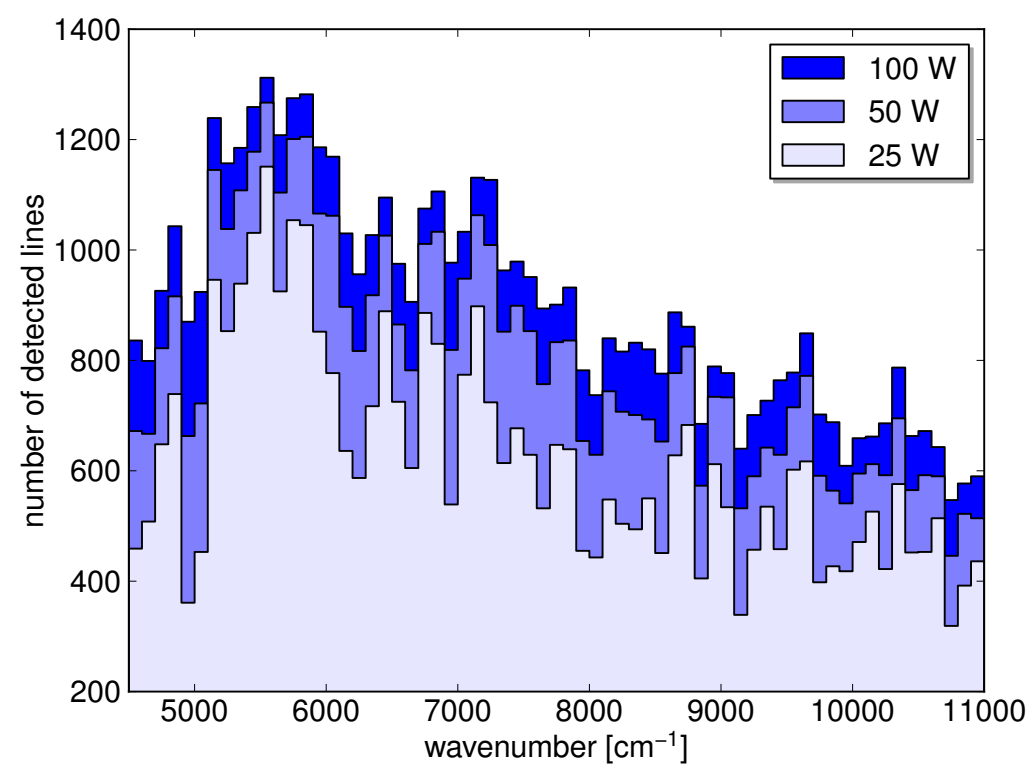

Figure 5.7: Histograms showing the distribution of emission lines as function of wavenumber. Each bin covers a range of $100 \mathrm{~cm}^{-1}$. The bars are not stacked and show the absolute number of lines in one bin for each of the three spectra operated at different $M W$ powers. (Boesch \& Reiners, 2015, Fig. 9)

In order to be useful for wavelength calibration, a spectrum needs a sufficient number of lines, depending on the instrument's resolution and wavelength coverage. Similar to Fig. 5.7, Fig. 5.8 shows the distribution of lines as a function of wavenumber, but this time each bin covers a constant number of 1000 resolution elements with a resolution of $R=\lambda / \Delta \lambda=100000$. This corresponds to specifications of high-resolution grating spectrographs like, e.g., CRIRES (Käufl et al., 2004). Considering the expected dynamic range of a near-infrared detector, in Fig. 5.8 we only include emission lines with peak intensities within two orders of magnitude: the histogram in the upper panel of Fig. 5.8 contains lines with flat-field corrected intensities of 0.5-50 (compare horizontal lines in Fig. 5.5). For example, at wavenumbers above $9000 \mathrm{~cm}^{-1}$, an average of about 0.4 lines fall into one resolution element. This number decreases toward smaller wavenumbers until very few lines of this intensity are available at $4500 \mathrm{~cm}^{-1}$. To detect more lines at smaller wavenumbers, we need to go to lower intensities as shown in the lower panel, which can be achieved using longer exposure times and a longpass filter. The second histogram contains lines with intensities $0.05-5$. Here, on average, there are approximately 0.5 lines per resolution element in the wavenumber range $5500-7750 \mathrm{~cm}^{-1}$. At wavenumbers above 
$7750 \mathrm{~cm}^{-1}$ (dashed line) fainter lines are missing in our measurements.

The continuous spread of more than 40000 detected lines over the wavenumber range $4500-11000 \mathrm{~cm}^{-1}$ ensures a sufficient number of lines for wavelength calibration. The exposure time might need to be adjusted when observing in different spectral regions to obtain the optimal number of lines, depending on a spectrograph's specific design. [See Sect. 5.2.3.4 for possible limitations due to blended lines at lower resolution.]

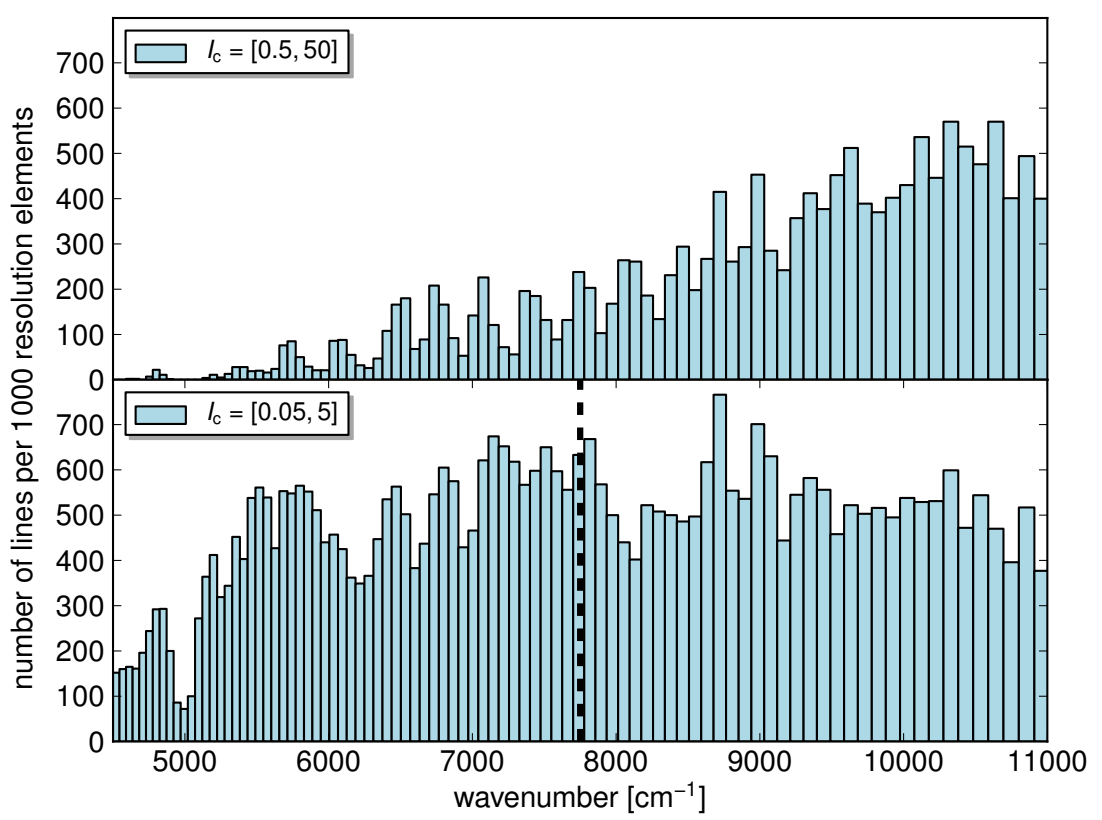

Figure 5.8: Histograms of detected emission lines as a function of wavenumber. The samples are limited to emission lines with a peak intensity 0.5 - 50 (upper panel) and $0.05-5$ (lower panel). Each bin covers 1000 resolution elements based on a resolution of $R=100000$. In the lower panel, the dashed vertical line is located at $7750 \mathrm{~cm}^{-1}$ and the sample is expected to be incomplete at higher wavenumbers (compare with Fig. 5.5). Data from discharge operated at $M W$ power of $50 \mathrm{~W}$. (Boesch 8 Reiners, 2015, Fig. 10)

\subsubsection{The influence of different MW powers on line width and intensity}

Varying the microwave power supplied to the cavity changes the mean power absorbed by electrons from the electric field and therefore the rate of collisional excitations in the plasma (Jankowski \& Reszke, 2011, chap. 1.2). Here we present how the widths of the spectral lines become broader with higher MW power and how the intensities of the atomic nitrogen lines increase with MW power.

Doppler broadening is expected to be the dominating effect on the line width after correction for instrumental line broading because of the low gas pressure and the Gaussian-shaped 
spectral lines. The theoretical description of Doppler broadening can be expressed as

$$
F W H M_{\mathrm{c}}=\sqrt{\frac{8 k T \ln (2)}{m c^{2}}} \tilde{\nu}_{0},
$$

where $k$ is the Boltzmann constant, $m$ is the mass of the species, and $c$ is the speed of light (e.g., Bernath, 2005, chap. 1.3). According to Eq. 5.1, lighter species create broader spectral lines compared to heavier species (at a constant temperature $T$ ), and the line width increases linearly with wavenumber $\tilde{\nu}_{0}$.

The $\mathrm{FWHM}_{\mathrm{c}}$ of all lines without a flag is plotted against wavenumber in Fig. 5.9. The identified lines of atomic and molecular nitrogen are highlighted. A linear regression using Eq. 5.1 is applied to the data points of each identified species, as represented by the lines in the respective colors in Fig. 5.9. The linear regression weights each point according to its uncertainty in $\mathrm{FWHM}_{\mathrm{c}}$, which can lead to a slight displacement of the linear regression relative to the bulk of the data. Atomic nitrogen clearly follows a trend of larger $\mathrm{FWHM}_{\mathrm{c}}$ as compared to molecular nitrogen. We also see the expected linear trend with wavenumber for all species. The distribution of the $\mathrm{FWHM}_{\mathrm{c}}$ from the unidentified lines is consistent with a combined $\mathrm{FWHM}_{\mathrm{c}}$ distribution of lines from $\mathrm{N}_{2}$ and $\mathrm{N}_{2}^{+}$with a ratio of $0.82: 0.18$, respectively. The overall scatter is, however, too large to make conclusive statements about the origins of individual lines.

Equation 5.1 includes a temperature parameter $T$. The definition of a single gas temperature is here not possible because a microwave induced plasma is not in a local thermodynamic equilibrium. Each species in the plasma rather has its own temperature (Jankowski \& Reszke, 2011, chap. 1.4). Because a change in temperature for a certain species is related to a change in FWHM, we compile the values of $T$ as derived from the linear regression for completeness in Table 5.3. The temperature rises for all species if the MW power is increased. The error on $T$, as obtained directly from the fit, is very small $(\leq 1 \mathrm{~K})$ because the linear regression consists of only one free parameter. To estimate more realistic uncertainties, we varied the data points by $\pm \sigma\left(\mathrm{FWHM}_{\mathrm{c}}\right)$ and report the resulting change in $T$ in Table 5.3 . 

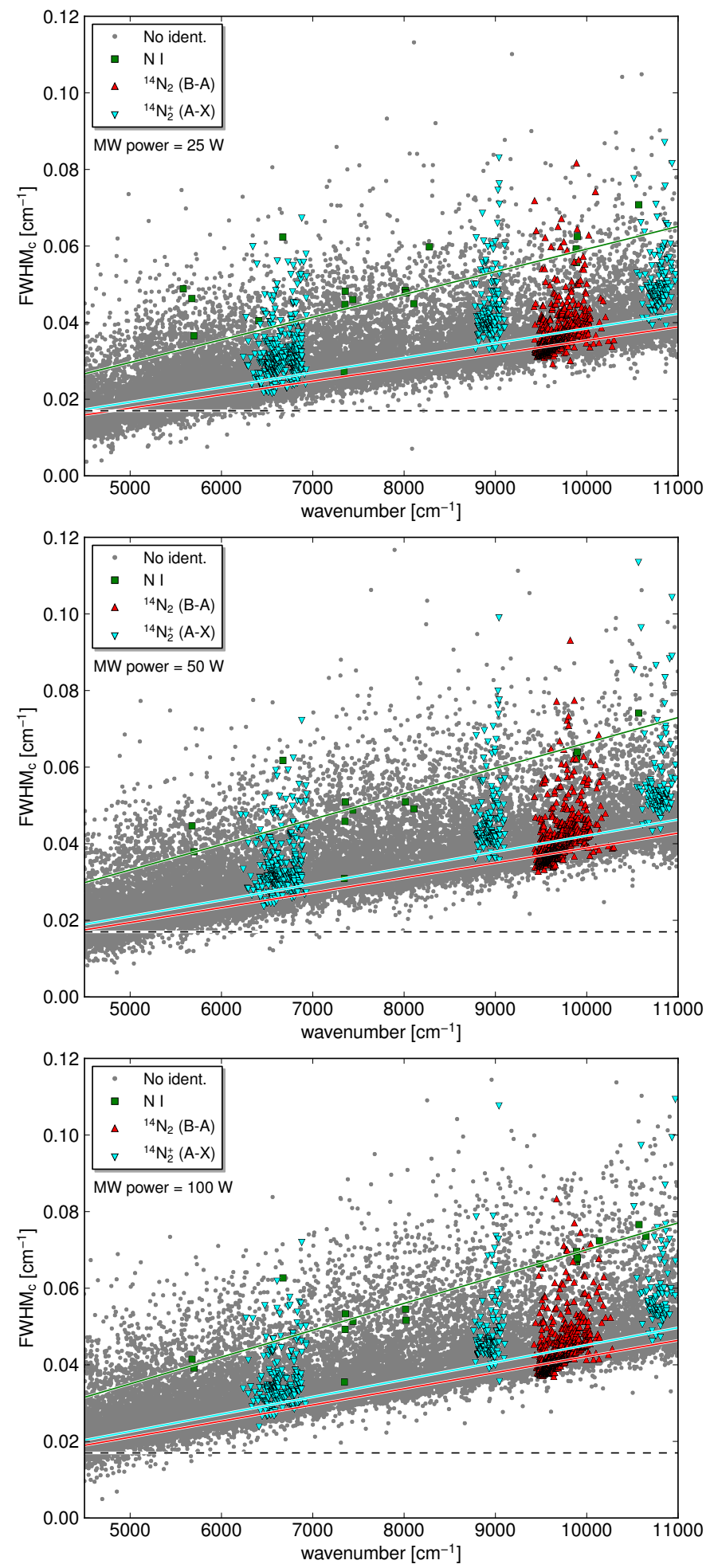

Figure 5.9: Each point represents the $F W H M_{c}$ of one spectral line as a function of wavenumber. The solid lines show linear regressions with ordinate crossing forced at the origin. The black dashed horizontal line indicates the FTS resolution. From top to bottom: discharge operated at microwave power of 25, 50, and $100 \mathrm{~W}$. (Boesch 8 Reiners, 2015, Fig. 11) 


\begin{tabular}{l|rrr}
\hline \hline & $\mathrm{N} \mathrm{I}$ & $\mathrm{N}_{2}{ }^{a}$ & $\mathrm{~N}_{2}^{+b}$ \\
\hline$T(25 \mathrm{~W})[\mathrm{K}]$ & $957 \pm 10$ & $681 \pm 11$ & $809 \pm 36$ \\
$T(50 \mathrm{~W})[\mathrm{K}]$ & $1200 \pm 2$ & $825 \pm 8$ & $967 \pm 27$ \\
$T(100 \mathrm{~W})[\mathrm{K}]$ & $1339 \pm 2$ & $970 \pm 7$ & $1113 \pm 20$ \\
\hline
\end{tabular}

Table 5.3: Values of the temperature parameter $T$ in Eq. 5.1 as derived by linear regression. Notes: ${ }^{(a)} 1$ band of the $B^{3} \Pi_{g}-A^{3} \Sigma_{u}^{+}$system. ${ }^{(b)} 4$ bands of the $A^{2} \Pi_{u}-X^{2} \Sigma_{g}^{+}$system. (Boesch E Reiners, 2015, Table 5)

Furthermore, we analyzed the change in peak intensities for the 25 transitions of $\mathrm{N}$ I that are identified in all three spectra. Figure 5.10 displays the mean change in peak intensity when the MW power is increased from $25 \mathrm{~W}$ to $50 \mathrm{~W}$ and then to $100 \mathrm{~W}$. The values are calculated in the following way: the intensity $I_{\mathrm{c}, P}$ of each $\mathrm{N}$ I line in the spectrum of the discharge operated at MW power $P$ is divided by the intensity $I_{\mathrm{c}, 25 \mathrm{~W}}$ of the same line in the spectrum of the discharge operated at $25 \mathrm{~W}$; then, the mean value is calculated. The latter appears to follow a linear trend as indicated by the dashed line in Fig. 5.10 and described by the following linear regression:

$$
\left\langle\frac{I_{\mathrm{c}, P}}{I_{\mathrm{c}, 25 \mathrm{~W}}}\right\rangle=0.0756 \frac{1}{\mathrm{~W}} \cdot P[\mathrm{~W}]-0.8679
$$

The individual intensity values are well separated into two disjointed distributions for the measurements with $50 \mathrm{~W}$ and $100 \mathrm{~W}$, with only one outlier at $7349 \mathrm{~cm}^{-1}$. If we remove this outlier, the error bars shrink by about a factor of two and the linear regression becomes $\left\langle\frac{I_{\mathrm{c}, P}}{I_{\mathrm{c}, 25 \mathrm{~W}}}\right\rangle=0.0781 \frac{1}{\mathrm{~W}} \cdot P[\mathrm{~W}]-0.9318$.

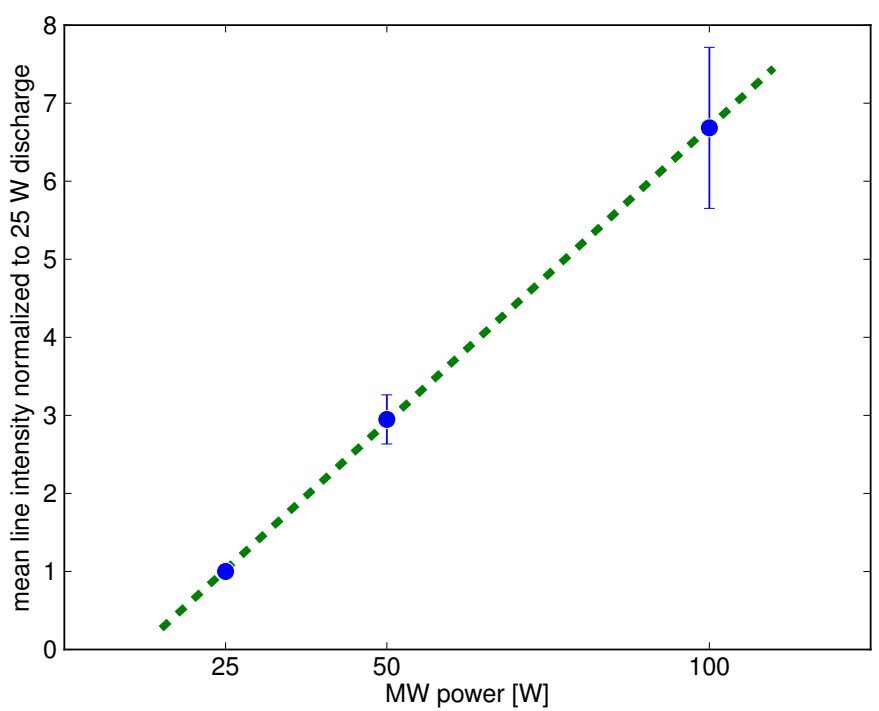

Figure 5.10: The mean intensity of the detected $N$ I lines relative to the respective line intensity in the spectrum of the discharge operated at $25 \mathrm{~W}$. The green dashed line indicates a linear regression. (Boesch 8 Reiners, 2015, Fig. 12) 


\subsubsection{The $\mathrm{CN}$ discharge cell ${ }^{6}$}

\subsubsection{Line identification}

We identify the emission lines in the spectrum to show qualitative properties of the different species, such as line distribution and line intensities. The empirical line identification is based on the distance of the spectral lines in our spectrum relative to observed and published line positions. For a match, we require that the absolute difference between a measured line position in our spectrum and a reference line from the literature is less than the line's half width at half maximum.

We assign transitions of the Red System $\mathrm{A}^{2} \Pi-\mathrm{X}^{2} \Sigma^{+}$of the CN molecule adopting a subset of the line list by Brooke et al. (2014): we use observed line positions from 20 vibrational bands that have more than 50 lines in the wavenumber interval between 4500 and $11000 \mathrm{~cm}^{-1}$. For the identification of lines from molecular nitrogen, we use the line list published in Boesch \& Reiners (2015) from the $\mathrm{N}_{2}$ discharge cell operated at a $\mathrm{MW}$ power of $50 \mathrm{~W}$. Furthermore, we checked line positions of atomic transitions (He I, C I, N I, O I and Ar I) using data from NIST (Kramida et al., 2014). In total, we could identify $87 \%$ of all detected lines with the above mentioned line lists. Only about $10 \%$ of the He I, O I, and Ar I transitions that are listed in the NIST database could be assigned to lines in our spectrum (12 lines in total). These lines are either misidentified and the corresponding species not present in the discharge spectrum, or only a few strong lines are above the detection threshold and just a very small amount of the corresponding species is present. The very small number of lines from argon, which is the third most abundant species in the atmosphere, indicates that the gas cell was properly filled and sealed. Of the other species, we assigned $4509 \mathrm{CN}$ lines, 25946 molecular nitrogen lines, $22 \mathrm{C}$ I lines, and 19 N I lines. 4474 lines remain unidentified.

Because the identification is solely based on line position, we expect that some lines are misidentified due to blends and noise, especially at low intensities. For the identified molecular species, we use the expected relative peak intensities of the spectral lines as a second criterion to check the overall consistency of the assignments.

For the molecular nitrogen lines, the comparison of measured and expected line intensities is relatively straightforward. The line list given in Boesch \& Reiners (2015) states observed line intensities that were determined under very similar experimental conditions. We assume that the temperature and therefore the relative line intensities are comparable to our new measurement. We show in Fig. 5.11 the correlation of line intensities in our spectrum with the corresponding intensities in the reference. The bulk of the data follows the expected perfect positive correlation. The orange line represents a fit to the data points that passes through the origin. We calculate a correlation coefficient $\operatorname{cov}(i, j) / \sqrt{\operatorname{cov}(i, i) \cdot \operatorname{cov}(j, j)}=0.94$, where $\operatorname{cov}(i, j)$ is the covariance matrix for two datasets $i$ and $j$. The scatter can be explained with an uncertainty in the gas temperature.

\footnotetext{
${ }^{6}$ Sections 5.2.3.1-5.2.3.3 have been submitted for publication to the scientific journal Astronomy 8 Astrophysics as Sect. 3.2.4 "Line identification", Sect. 3.3 "Line intensity" and Sect. 3.4 "Line density" in "Near-infrared wavelength calibration with molecular discharge spectra of nitrogen and CN".
} 
Most outliers occur in the upper left part of the figure, i.e., the measured lines are stronger than their assigned lines from the reference. This can be caused by blends with other spectral lines.

For the CN lines, we used the molecular constants given by Brooke et al. (2014) to model the vibrational bands with the program PGOPHER. For the simulation, we used a temperature of $T_{0}=943 \mathrm{~K}$ that we estimated from the widths of the CN lines after correcting for instrumental broadening. The measured intensities of the lines are plotted against calculated intensities in Fig. 5.12. The size of each point represents the rotational quantum number of the lower energy level, $J^{\prime \prime}$, with larger point sizes for decreasing $J^{\prime \prime}$. As for the molecular nitrogen lines in Fig. 5.11, the bulk of the data in Fig. 5.12 follows a strong positive correlation; the orange line indicates again a linear regression. The correlation coefficient for the data shown in Fig. 5.12 is 0.96 . However, data points from the $0-0,0-1$, and $0-2$ vibrational bands also show a large deviation from the expected trend for higher $J^{\prime \prime}$ values. This deviation is not random but contains structure. The structure becomes more visible when the temperature of the simulation is changed from $T_{0}$ to higher or lower values: in these cases, all data points shift away from the orange line and the points from each vibrational band follow a distinct progression. Because all lines from one band follow the same progression, we believe that the lines are correctly identified as $\mathrm{CN}$, and assume that the theoretical treatment of intensities for spectral lines with high rotational quantum numbers is less accurate compared to the transitions with lower $J^{\prime \prime}$. We conclude that the observed scatter in Figs. 5.11 and 5.12 is acceptable for identifying lines for the qualitative analysis of line intensities and line density.

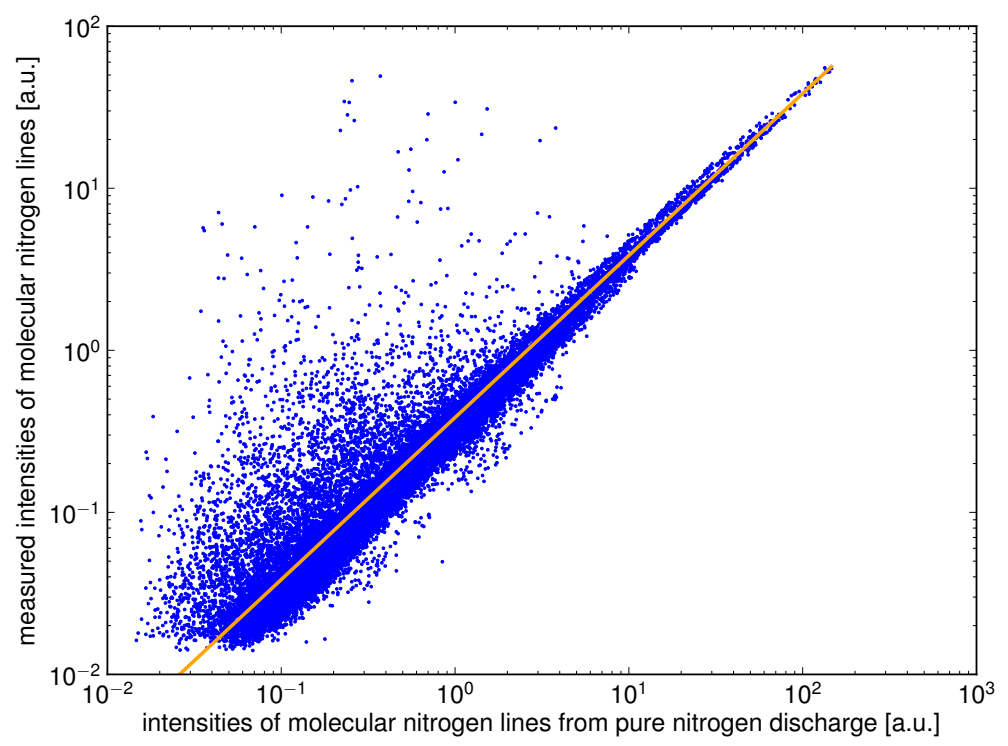

Figure 5.11: Correlation between flat-field corrected peak intensities of assigned molecular nitrogen lines in the spectrum of the $C N$ discharge cell and the flat-field corrected peak intensities of spectral lines from the $\mathrm{N}_{2}$ discharge cell as published in Boesch 8 Reiners (2015). The orange line represents a linear regression to the data points passing through the origin. 


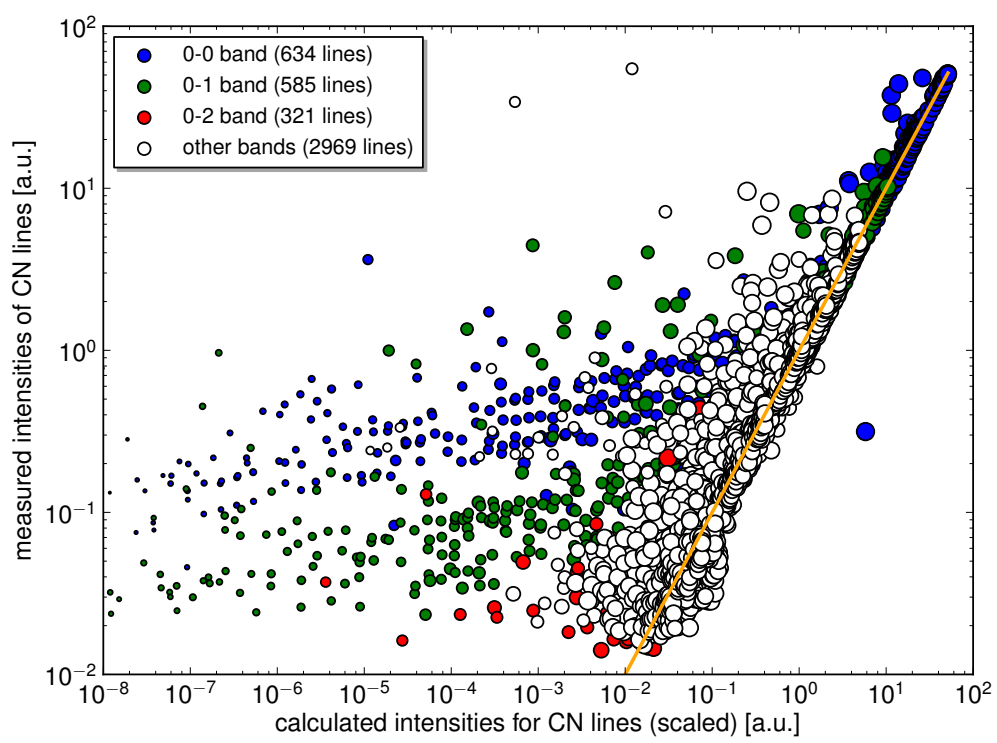

Figure 5.12: Correlation between flat-field corrected peak intensities of assigned CN lines in the spectrum of the $C N$ discharge cell and calculated line intensities. The line intensities are calculated with the program PGOPHER individually for each vibrational band and then scaled to the intensities of the observed values. Larger points correspond to smaller vibrational quantum numbers $J^{\prime \prime}$. The orange line represents a linear regression to the data points and through the origin.

\subsubsection{Line intensity}

We show in Fig. 5.13 the flat-field corrected line intensities as a function of wavenumber. Each point represents one detected spectral line. We highlight the assigned CN lines with larger red points. The overall structure of the plot looks similar to Fig. 5.5 for the $\mathrm{N}_{2}$ discharge cell: the detection limit at lower intensities follows the shape of the flat-field curve and the detection sensitivity is better at smaller wavenumbers compared to larger wavenumbers. The dynamic range of the detected lines after flat-field correction covers three to four orders of magnitude. In total, $11 \%$ of the lines have an intensity larger than 1 a.u., $46 \%$ of the line intensities fall into the region between 0.1 a.u. and 1 a.u., and $43 \%$ of the lines have an intensity of 0.1 a.u. and less. Compared to the pure $\mathrm{N}_{2}$ discharge, the CN molecules add additional vibrational bands. In general, these CN bands have similar band intensities as the nitrogen bands. For wavelength calibration, these additional lines can bring both advantages and disadvantages: on the one hand, more lines add RV information to the spectrum; on the other hand, an increasing number of blended lines, especially at low intensities, may be an obstacle during the wavelength calibration process.

\subsubsection{Line density}

In Fig. 5.14, we show a histogram of the number of spectral lines per wavenumber bin. The bin width corresponds to 1000 resolution elements based on a resolving power of 


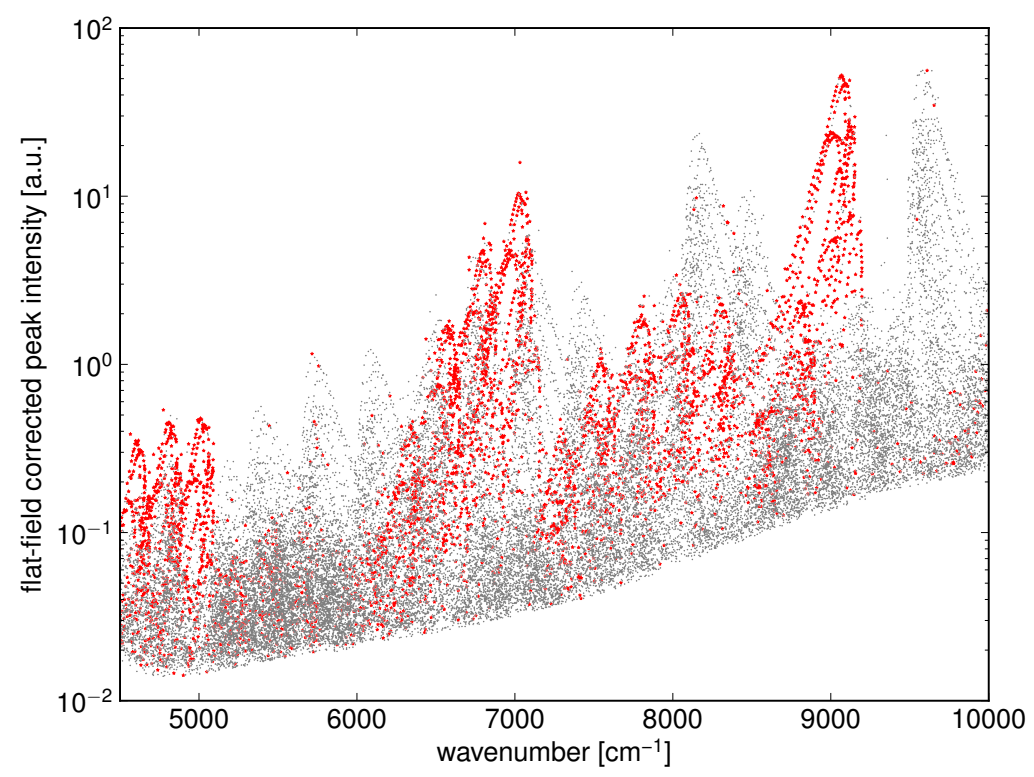

Figure 5.13: Flat-field corrected line intensities as a function of wavenumber. Each point represents one spectral line. The assigned $C N$ lines are highlighted as larger points with red color.

$R=100000$. Consequently, the bin width increases from $45 \mathrm{~cm}^{-1}$ at $4500 \mathrm{~cm}^{-1}$ to $100 \mathrm{~cm}^{-1}$ at $10000 \mathrm{~cm}^{-1}$. Identified molecular nitrogen and $\mathrm{CN}$ lines are indicated separately. Not identified lines and atomic lines are combined under the label "not ident./other" for clarity. An average of approximately $80 \mathrm{CN}$ lines is located within one bin in the spectral range between $6500 \mathrm{~cm}^{-1}$ and $9000 \mathrm{~cm}^{-1}$. This are about four times less lines from $\mathrm{CN}$ than from molecular nitrogen in this region. At wavenumbers below $5000 \mathrm{~cm}^{-1}$, an average of approximately $60 \mathrm{CN}$ lines is located in one bin; about half as many lines as from molecular nitrogen on average. Only very few $\mathrm{CN}$ lines are present past the $0-0$ band at wavenumbers above approximately $9200 \mathrm{~cm}^{-1}$. The distribution of molecular nitrogen lines is similar to the $\mathrm{N}_{2}$ discharge, as discussed in detail in Sect. 5.2.2, however, with approximately 150 fewer lines per bin, mostly because of missing faint lines.

In total, 34982 spectral lines were determined in the spectrum, including $4509 \mathrm{CN}$ lines and 25946 molecular nitrogen lines (see line identification in Sect. 5.2.3.1). For comparison, line lists for HCLs, which are often used for wavelength calibration of astronomical spectrographs, contain about 7000 lines for uranium (Redman et al., 2011) and about 3600 lines for thorium (Redman et al., 2014) in the same spectral region. We include the number of uranium and thorium lines per wavenumber bin in Fig. 5.14 as represented by the solid and dashed lines, respectively. The number of emission lines from the CN molecule alone does not raise the number of documented lines, as compared to HCLs. However, when the emission lines from molecular nitrogen are included, we obtain about four times more lines as compared to uranium in HCLs. It is noteworthy that the observations of the different lamps were all carried out under different experimental conditions. Therefore, the number of lines stated above are not necessarily the true number of lines provided by each source, and not all lines might be usable for wavelength calibration depending on 
the properties (e.g., resolution, sensitivity) of a spectrograph. The stated numbers should rather be understood as an overview over the quantity of currently documented lines under certain experimental conditions.

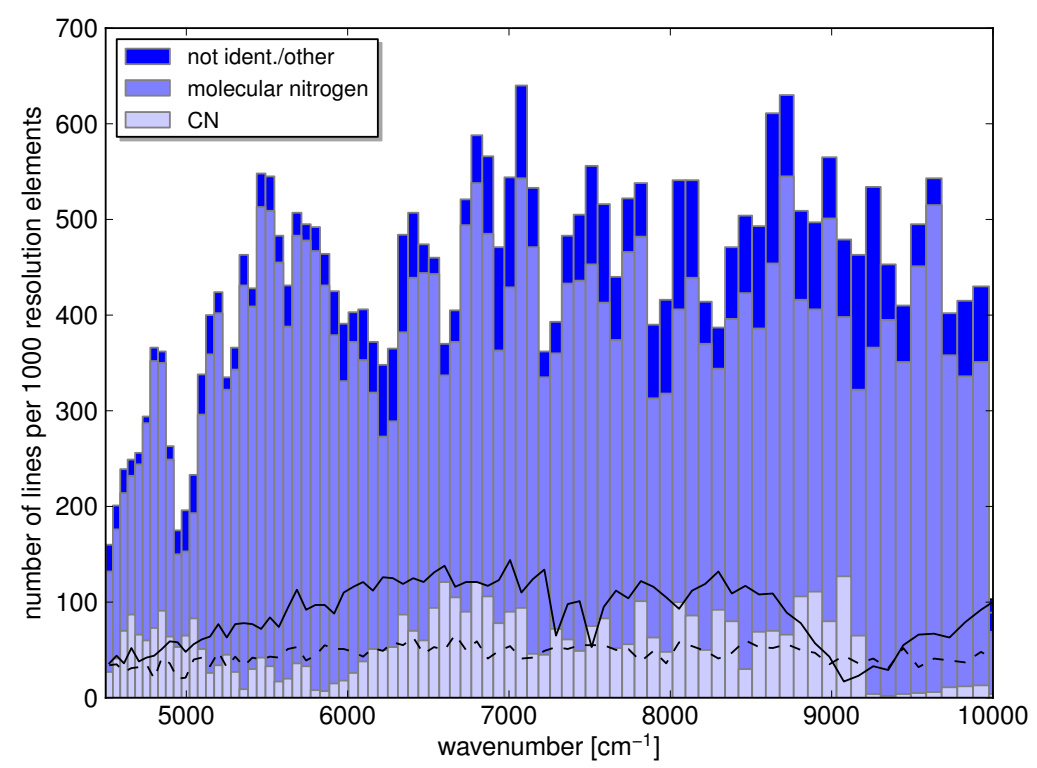

Figure 5.14: A stacked histogram showing the number of spectral lines as a function of wavenumber. Each bin covers 1000 resolution elements based on a resolving power of $R=100000$. The category "not ident./other" includes spectral lines that could not be assigned to molecular nitrogen or CN, and the relatively few identified atomic spectral lines. The solid and dashed lines indicate the number of uranium and thorium lines as derived from spectra of HCLs by Redman et al. (2011, 2014), respectively.

\subsubsection{Isolated lines in a limited dynamic range}

The emission lines in the discharge spectrum show inhomogeneities regarding line spacing and line intensities. Many lines are blended at the high resolution achievable with the FTS: $42 \%$ of the lines have a neighboring line that is closer than the sum of the two lines' FWHM. Even more lines will overlap when the spectrum is recorded with a spectrograph of lower resolution. Therefore, it can be expected that a large number of lines will not be usable for wavelength calibration of astrophysical spectrographs. It is very difficult to make a general statement concerning the number of usable lines that accounts for all possible characteristics of instruments and wavelength ranges. However, using a few assumptions, I try to estimate the number of lines that are not blended at a certain spectral resolution and that have an intensity within a limited dynamic range. A comparison with HCLs that is universally valid is also difficult because the published lines lists for these lamps have been measured with different spectrographs and at different operating currents.

A number of assumptions are made for the following treatment. First, a detector used for an astrophysical spectrograph shall distinguish spectral lines over a dynamic range 
of two orders of magnitude. In order not to saturate any pixels of the detector, an exposure time is chosen so that the strongest line is just below the saturation limit. In the recorded spectrum of the $\mathrm{CN}$ cell, the strongest line has a flat-field corrected intensity of $I_{\mathrm{c}}=55.63$ a.u. (compare Fig. 5.13). Thus, all lines with intensities between 0.56 a.u. and 55.63 a.u. are considered. At wavenumbers below $6500 \mathrm{~cm}^{-1}$, only very few lines fall into this intensity range (compare again Fig. 5.13) and the wavenumber range $4500-6500 \mathrm{~cm}^{-1}$ is treated separately. The maximum intensity is $I_{\mathrm{c}}=2.04 \mathrm{a} . \mathrm{u}$. in the spectral range below $6500 \mathrm{~cm}^{-1}$. The second assumption is that the position of a line is not significantly influenced by blends with other lines if it is isolated under the following conditions ("isolated line"): no neighboring line, that is closer than three resolution elements, has a relative peak intensity stronger than $10 \%$. A resolution element shall be equal to the FWHM of the unresolved lines. Comparing two overlapping Gaussian profiles (same FWHM, peak intensity ratio of 0.1 ) at different separations, it can be shown that the maximum shift of the stronger line's peak position is less than three percent of its FWHM. This shift is comparable to the measurement uncertainty of the peak position in FTS spectra. In a similar test, it can be shown that stronger neighboring lines with a separation larger than three resolution elements do not influence the peak position, taking into account that the intensity range is already limited to two orders of magnitude.

I show in Fig. 5.15 the number of lines from the spectrum of the CN cell that fulfill the above mentioned criteria. The bin width of the histogram is $250 \mathrm{~cm}^{-1}$ and the results are shown for three different spectral resolving powers $R=125000, R=100000$ and $R=75000$. It can be seen that the number of lines per bin increases with higher spectral resolution. Increasing the resolving power from $R=75000$ by a factor of 1.67 to $R=125000$ raises the number of lines in a bin by a factor of 2 to 6 .

For better comparison with Fig. 5.14, I show in Fig. 5.16 the histogram with bin widths corresponding to resolution elements based on a resolving power of $R=100000$. The histogram bars represent the number of lines in the spectrum of the CN cell that fulfill the above mentioned criteria for isolated lines based also on a resolving power of $R=100000$. The line intensities are again limited to the same values as in Fig. 5.15 with a split at $6500 \mathrm{~cm}^{-1}$. Comparing with Fig. 5.14, the number of lines in a bin has decreased on average by $85 \%$ and $95 \%$ for the spectral ranges below and above $6500 \mathrm{~cm}^{-1}$, respectively. Less than 10 lines are left in a few bins. The spectral ranges with a higher number of spectral lines (e.g., around $7000 \mathrm{~cm}^{-1}, 8200 \mathrm{~cm}^{-1}$ and $9700 \mathrm{~cm}^{-1}$ ) correspond to the strongest vibrational bands of $\mathrm{N}_{2}$ and $\mathrm{CN}$ which can be seen in the raw spectrum shown in Fig. 5.3.

The distribution of uranium and thorium lines is also shown in Fig. 5.16 using data from the line lists by Redman et al. (2011) and Kerber et al. (2008), respectively. The lines of uranium and thorium are filtered in a similar way as the emission lines of the discharge spectrum. First, the lines with the strongest intensities within two orders of magnitude are selected within the two spectral ranges $4500-6500 \mathrm{~cm}^{-1}$ and $6500-10000^{-1}$. Second, the "isolated lines" are selected as described above for the lines of the CN cell's spectrum. After this selection process for the spectral lines, the discharge spectrum still provides more emission lines in almost all wavenumber bins. Note that spectral lines from the filling gas in the HCL are not considered. In practice, they can account for many of the strongest lines 


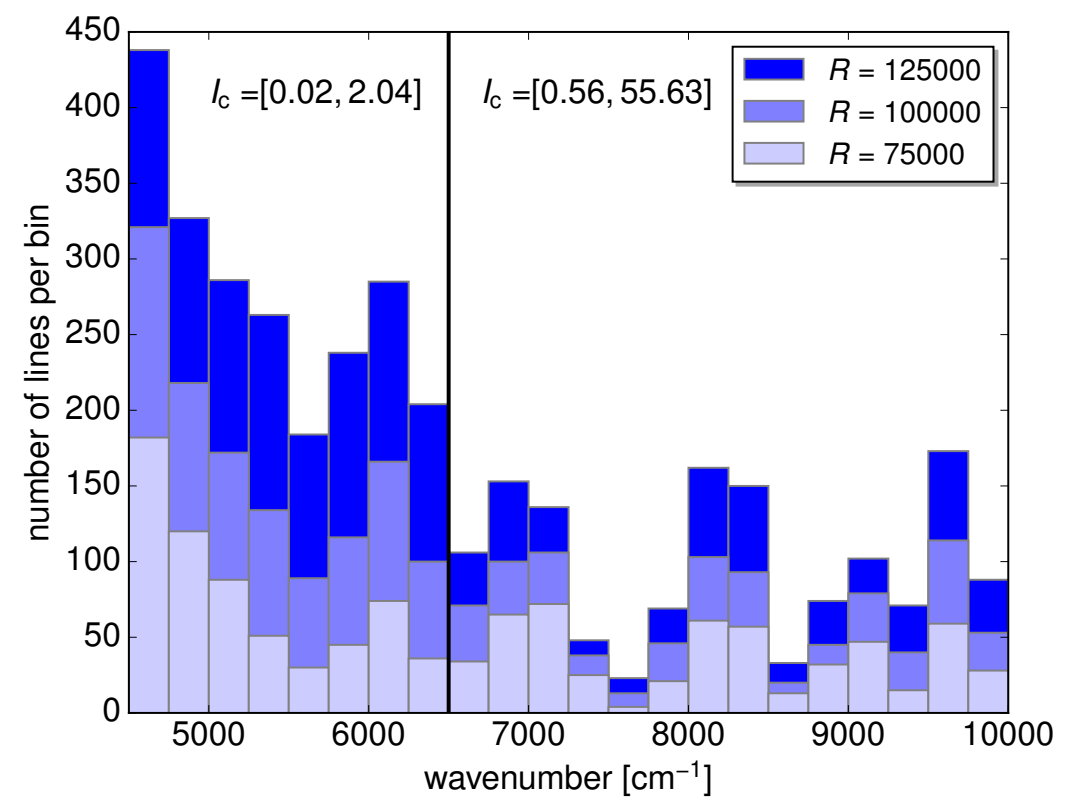

Figure 5.15: Histogram showing the number of isolated spectral lines (see main text for definition) in the spectrum of the $C N$ cell as a function of wavenumber for three different resolving powers $R$. The bin width is fixed to $250 \mathrm{~cm}^{-1}$. In the spectral range $4500-6500 \mathrm{~cm}^{-1}$, the histogram only contains lines with flat-field corrected intensities in the range $I_{c}=[0.02,2.04]$ and in the spectral range $6500-10000 \mathrm{~cm}^{-1}$ it shows only lines with intensities in the range $I_{c}=[0.56,55.63]$.

in the spectrum. For example, in the line list by Kerber et al. (2008) for a thorium-argon HCL operated at $20 \mathrm{~mA}$, the 43 strongest lines in the spectral range $4500-10000 \mathrm{~cm}^{-1}$ originate from argon. The intensities of these argon lines are up to 30 times higher than the strongest thorium line. The strong lines from the filling gas saturate the detector if the exposure time is not adjusted. Another note concerns the operating current of the uranium lamps that have been used to create the line list by Redman et al. (2011): the lamps were operated at currents of up to $300 \mathrm{~mA}$ which is considerably higher than the current used for HCL in wavelength calibration (around $10 \mathrm{~mA}$ ). Taking these considerations into account, less lines from HCLs might be usable for wavelength calibration as the ones shown in Fig. 5.16. 


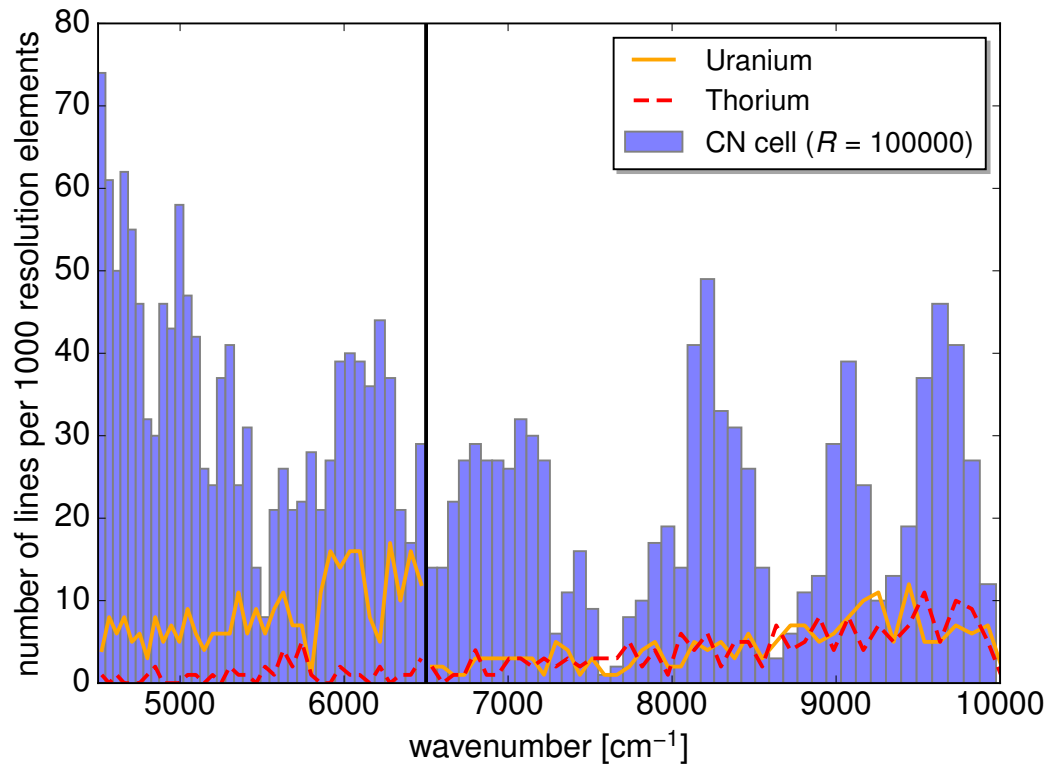

Figure 5.16: Histogram showing the number of isolated spectral lines (see main text for definition) in the spectrum of the CN cell and for species used in HCLs as a function of wavenumber. The bin width corresponds to 1000 resolution elements based on a resolving power of $R=100000$. See main text for the intensities of the considered spectral lines and the split at $6500 \mathrm{~cm}^{-1}$.

\subsection{Stability of line positions ${ }^{7}$}

In order to be useful as a wavelength calibrator in precision spectroscopy, a calibration source must provide a spectral output with stable line positions. In this section, we analyze the shifts in the positions of the emission lines over time. The FTS uses an internal stabilized laser to establish the wavelength scale of the recorded spectra. However, this wavelength solution is not sufficient to reliably measure shifts on the $\mathrm{m} / \mathrm{s}$ level because it varies with changing environmental conditions. Therefore, we simultaneously record the absorption spectrum of a gas cell filled with acetylene $\left(\mathrm{C}_{2} \mathrm{H}_{2}\right)$ that serves as a relative wavelength reference. The $\mathrm{C}_{2} \mathrm{H}_{2}$ gas cell is illuminated by a halogen lamp [compare the experimental setup shown in Fig. 4.4].

\subsubsection{Measurement procedure}

The stability measurements for the $\mathrm{N}_{2}$ cell and the CN cell were carried out on 31 May and 2 June 2015, respectively. We recorded one spectrum every 30 minutes over 24 hours of the combined light of the MW powered discharge and the absorption cell reference (resulting in a total of 48 spectra). Figure 5.17 shows one of these spectra from the measurements with the $\mathrm{N}_{2}$ discharge cell. Each spectrum is an average of 50 scans with a total scanning time

\footnotetext{
${ }^{7}$ This section has been submitted for publication to the scientific journal Astronomy 83 Astrophysics as Sect. 4 "Stability of line positions" in "Near-infrared wavelength calibration with molecular discharge spectra of nitrogen and CN".
} 
of approximately 25 minutes. We used a resolution of $0.05 \mathrm{~cm}^{-1}$ and an aperture of $1.3 \mathrm{~mm}$. Experience has shown that we obtain a sufficient signal-to-noise with these parameters for both light sources, yet still allowing a reasonable time resolution. The vacuum pump of the FTS was activated about ten hours before the first measurement to create a stable pressure of $\leq 0.1$ mbar inside the spectrometer.

After the 24 hour time series, templates were recorded for each channel: one template spectrum of only the discharge and one template of only the halogen lamp with the $\mathrm{C}_{2} \mathrm{H}_{2}$ cell. The templates were recorded with twice the number of scans (100 scans) in order to obtain a higher signal-to-noise.

The spectral data shown in Fig. 5.17 covers the range $4000-10000 \mathrm{~cm}^{-1}$. Because of the shape of the black-body and the contamination from atmospheric water, only the spectral regions $5600-6700 \mathrm{~cm}^{-1}$ and $7500-10000 \mathrm{~cm}^{-1}$ are used in the stability analysis (highlighted in green in Fig. 5.17).

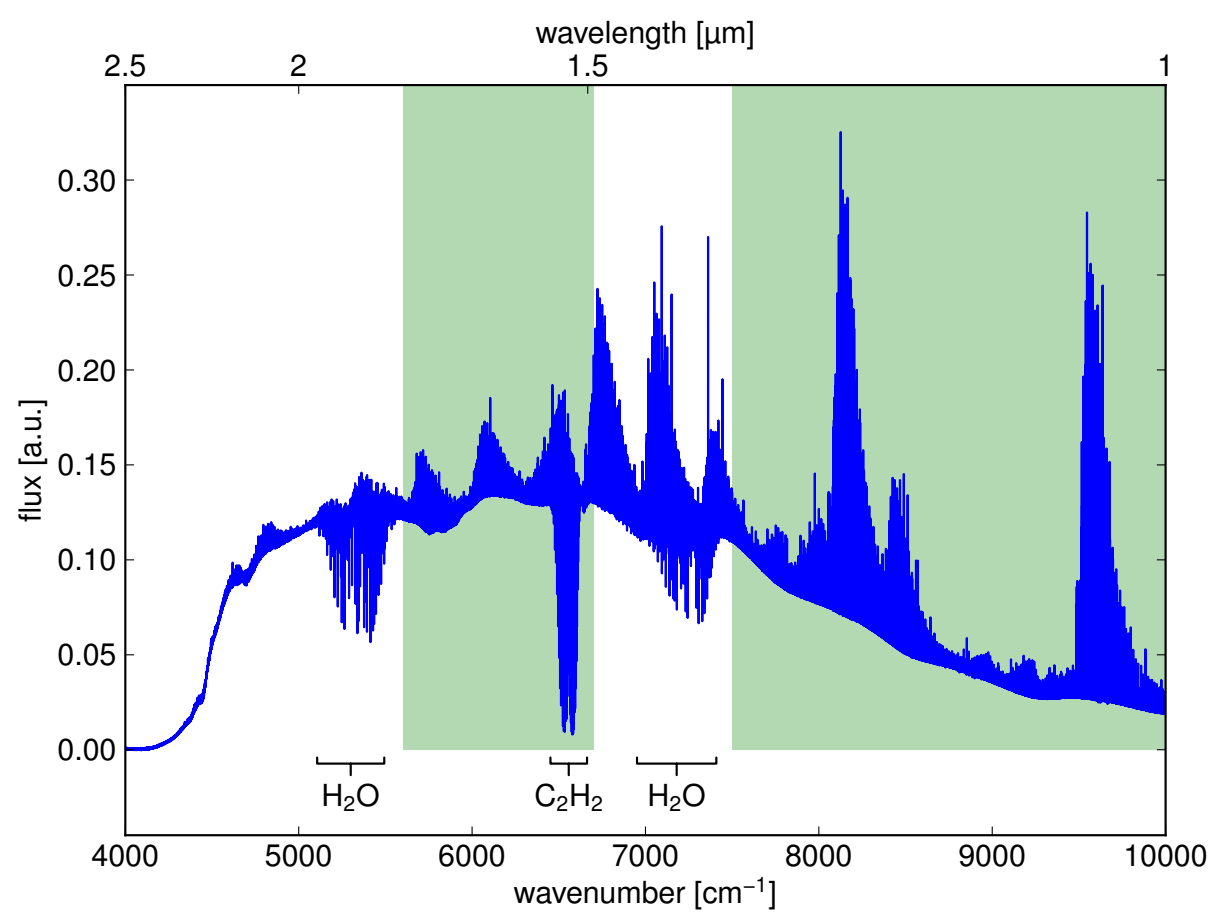

Figure 5.17: One spectrum from the RV stability test time series of the combined light of the $N_{2}$ discharge cell (emission lines) and the $\mathrm{C}_{2} \mathrm{H}_{2}$ reference cell (black body contribution from halogen lamp and absorption lines). Additional absorption comes from atmospheric water. The spectral parts used for the stability analysis are shadowed in green color.

\subsubsection{Algorithm for measuring relative radial velocity shifts}

The apparent RV shifts of the two components (discharge lamp and absorption reference) in the combined spectrum need to be disentangled. We use a modified version of the 
algorithm presented by Anglada-Escudé \& Butler (2012) [see Sect. 2.4.2 for a full analytic description of the modified algorithm]. Given the template of the discharge spectrum $F_{\mathrm{MW}}$ and the template of the absorption reference $F_{\mathrm{C}_{2} \mathrm{H}_{2}}$, we want model the observed spectra $f_{\text {com }}$ with the two combined sources from the 24 hours time series. Adopting the notation of Anglada-Escudé \& Butler (2012), we define the difference $R$ between the templates and a spectrum from the time series at each wavelength $\lambda$ as

$$
\begin{aligned}
R[\lambda, \hat{\alpha}] & =F_{\mathrm{MW}}\left[\alpha_{\mathrm{MW}, \mathrm{v}} \lambda\right] \cdot \sum_{m=0}^{M} \alpha_{\mathrm{MW}, m}\left(\lambda-\lambda_{\mathrm{c}}\right)^{m} \\
& +F_{\mathrm{C}_{2} \mathrm{H}_{2}}\left[\alpha_{\mathrm{C}_{2} \mathrm{H}_{2}, \mathrm{v}} \lambda\right] \cdot \sum_{m=0}^{M} \alpha_{\mathrm{C}_{2} \mathrm{H}_{2}, m}\left(\lambda-\lambda_{\mathrm{c}}\right)^{m}-f_{\mathrm{com}}[\lambda] .
\end{aligned}
$$

In addition to fitting wavelength shifts, we used one polynomial for each template to correct for intensity changes. The subtraction of the central wavelength $\lambda_{\mathrm{c}}$ helps to avoid numerical problems with large numbers. A linear polynomial was used in our analysis $(M=1)$, resulting in the set of fitting parameters $\hat{\alpha}=\left\{\alpha_{\mathrm{MW}, \mathrm{v}}, \alpha_{\mathrm{MW}, 0}, \alpha_{\mathrm{MW}, 1}, \alpha_{\mathrm{C}_{2} \mathrm{H}_{2}, \mathrm{v}}, \alpha_{\mathrm{C}_{2} \mathrm{H}_{2}, 0}, \alpha_{\mathrm{C}_{2} \mathrm{H}_{2}, 1}\right\}$. The RV shifts for the two templates $F_{\mathrm{MW}}$ and $F_{\mathrm{C}_{2} \mathrm{H}_{2}}$ can be extracted using the expressions $v_{\text {rad, } \mathrm{MW}}=c \cdot\left(\alpha_{\mathrm{MW}, \mathrm{v}}-1\right)$ and $v_{\mathrm{rad}, \mathrm{C}_{2} \mathrm{H}_{2}}=c \cdot\left(\alpha_{\mathrm{C}_{2} \mathrm{H}_{2}, \mathrm{v}}-1\right)$, respectively. We drop the indices $\mathrm{MW}$ and $\mathrm{C}_{2} \mathrm{H}_{2}$ where it is clear from the context which $\mathrm{RV}$ is presented. The forward modeling is an iterative process that tries to minimize the following equation

$$
\chi^{2}=\sum_{i}^{N} \omega\left(R\left[\lambda_{i}, \hat{\alpha}\right]\right)^{2},
$$

with the sum over all $N$ wavelengths (data points) in the spectrum. The weight $\omega$ is the same at all wavelengths and is calculated from the noise in the three spectra $F_{\mathrm{MW}}, F_{\mathrm{C}_{2} \mathrm{H}_{2}}$ and $f_{\text {com }}$, namely $\omega=\left(\sigma\left(F_{\mathrm{MW}}\right)^{2}+\sigma\left(F_{\mathrm{C}_{2} \mathrm{H}_{2}}\right)^{2}+\sigma\left(f_{\text {com }}\right)^{2}\right)^{-1}$. The noise values $\sigma(f)$, with $f=\left\{F_{\mathrm{MW}}, F_{\mathrm{C}_{2} \mathrm{H}_{2}}, f_{\mathrm{com}}\right\}$, were determined as described in Sect. 3.4.1 using the spectral region $4000-4100 \mathrm{~cm}^{-1}$. The errors of the final fit parameters are calculated from the covariance matrix of the modeling algorithm (see Anglada-Escudé \& Butler, 2012), which are then propagated to calculate the errors of the radial velocities.

\subsubsection{Limits on radial velocity precision}

The precision of the RV measurement is limited by two factors: first, the RV information content of the spectrum depending on the shape of the spectrum and the noise in the observation; second, the wavelength stability of the $\mathrm{C}_{2} \mathrm{H}_{2}$ reference spectrum.

\subsubsection{Radial velocity information content}

The fundamental limit to RV measurements due to the noise in a spectrum has been analyzed, e.g., by Connes (1985), Butler et al. (1996) and Bouchy et al. (2001). The uncertainty of the RV depends on the number of data points $N$, the gradient of the 


\begin{tabular}{lc}
\hline \hline spectrum type & RV uncertainty $\sigma\left(v_{\text {rad }}\right)$ \\
\hline $\mathrm{C}_{2} \mathrm{H}_{2}$ cell template & $0.31 \mathrm{~m} / \mathrm{s}$ \\
$\mathrm{N}_{2}$ discharge cell template & $0.04 \mathrm{~m} / \mathrm{s}$ \\
$\mathrm{N}_{2}$ discharge cell $+\mathrm{C}_{2} \mathrm{H}_{2}$ cell & $0.08 \mathrm{~m} / \mathrm{s}$ \\
\hline $\mathrm{C}_{2} \mathrm{H}_{2}$ cell template & $0.38 \mathrm{~m} / \mathrm{s}$ \\
$\mathrm{CN}$ discharge cell template & $0.06 \mathrm{~m} / \mathrm{s}$ \\
$\mathrm{CN}$ discharge cell $+\mathrm{C}_{2} \mathrm{H}_{2}$ cell & $0.15 \mathrm{~m} / \mathrm{s}$ \\
\hline
\end{tabular}

Table 5.4: Uncertainty in the RV for the different types of spectra as calculated with Eq. 5.5. Notes: ${ }^{(a)}$ An individual template for the $\mathrm{C}_{2} \mathrm{H}_{2}$ cell was recorded for each of the two $\mathrm{RV}$ measurement runs.

spectrum at each data point (i.e., the sharpness of the spectral lines) and the noise $\sigma(f)$ :

$$
\sigma\left(v_{\mathrm{rad}}\right)=c \cdot\left(\sqrt{\sum_{i}^{N}\left(\frac{\lambda_{i} \cdot\left(\partial f_{i} / \partial \lambda_{i}\right)}{\sigma(f)}\right)^{2}}\right)^{-1}
$$

where $c$ is the speed of light, $f_{i}$ the intensity and $\lambda_{i}$ the wavelength (or wavenumber) at data point $i$ [see Sect. 2.5 for a derivation of Eq. 5.5].

Table 5.4 lists the calculated RV uncertainties for the combined spectral regions 5600 $6700 \mathrm{~cm}^{-1}$ and $7500-10000 \mathrm{~cm}^{-1}$ using Eq. 5.5. The RV uncertainty is highest for the $\mathrm{C}_{2} \mathrm{H}_{2}$ absorption template spectrum: although the $\mathrm{C}_{2} \mathrm{H}_{2}$ lines are very sharp, the small number of lines in a limited spectral range (compare Fig. 5.17) keeps the RV accuracy at $31 \mathrm{~cm} / \mathrm{s}$ and $38 \mathrm{~cm} / \mathrm{s}$ for the two measurements. The lowest uncertainty is calculated for the two template spectra of the discharge cells. These spectra have many sharp emission lines covering a large spectral range. In comparison to the discharge template, the RV uncertainty increases in the combined spectrum of the discharge and the absorption spectrum of the $\mathrm{C}_{2} \mathrm{H}_{2}$ cell. This is caused by the relatively high integrated flux produced by the halogen lamp which raises the noise level (Davis et al., 2001).

\subsubsection{Precision of $\mathrm{C}_{2} \mathrm{H}_{2}$ spectrum}

The absorption lines of ${ }^{12} \mathrm{C}_{2} \mathrm{H}_{2}$ around $6557 \mathrm{~cm}^{-1}(1525 \mathrm{~nm})$ are a NIST standard for wavelength calibration with line positions measured accurately to a few tens of meters per second (Gilbert \& Swann, 2001). We shortly discuss some external influences on the line positions. The pressure-induced shift of the lines was investigated by Swann \& Gilbert (2000), including the effect of temperature and electromagnetic fields. The latter is interesting, because the absorption cell is in close proximity of the MIP lamp. However, the regime in which high-intensity ac electric fields can split and shift line centers starts at $8 \cdot 10^{8} \mathrm{~W} / \mathrm{cm}^{2}$ for a gas pressure of $100 \mathrm{mbar}$. This is far beyond the intensity measured at the position of the acetylene cell in our setup $\left(<4 \mathrm{~mW} / \mathrm{cm}^{2}\right)$.

Variations in temperature can influence the pressure shift by changing the collision frequency. According to Swann \& Gilbert (2000), this effect is proportional to the gas density (which is constant in our case) and the square root of the temperature. The temperature-induced 
pressure shift $\Delta \tilde{\nu}(T)$ can be written as

$$
\Delta \tilde{\nu}(T)=\Delta \tilde{\nu}\left(T_{m}\right) \sqrt{T / T_{m}}
$$

where $\Delta \tilde{\nu}\left(T_{m}\right)$ is the pressure shift measured at a reference temperature $T_{m}$; temperatures are given in Kelvin. The temperature in the laboratory was constantly measured and stayed nearly constant at $(21.6 \pm 1){ }^{\circ} \mathrm{C}$. The temperature inside the Faraday cage of the experiment increased as soon as the MIP lamp was switched on. The available temperature recordings and experience from other measurements suggest that the temperature inside the Faraday cage did not exceed $35^{\circ} \mathrm{C}$. The actual temperature of the gas inside the absorption tube at a given time is unknown, but using Eq. 5.6 we can estimate an upper limit for the temperature dependent pressure shift. For the temperature difference of $20.6{ }^{\circ} \mathrm{C}$ to $35^{\circ} \mathrm{C}$, the shift is about $1.42 \mathrm{~m} / \mathrm{s}$. A relative RV shift of up to $1.42 \mathrm{~m} / \mathrm{s}$ between the discharge spectrum and the $\mathrm{C}_{2} \mathrm{H}_{2}$ spectrum can therefore be explained by the temperature dependence of the reference spectrum. The observed relative shift can be smaller if the gas inside the cell does not heat up to the ambient air temperature or if the theoretical temperature dependence is overestimated.

\subsubsection{Radial velocity stability}

\subsubsection{Global stability of the $\mathrm{N}_{2}$ cell and the $\mathrm{CN}$ cell}

We show the results of the RV stability tests for the $\mathrm{N}_{2}$ cell and the CN cell in Figs. 5.18 and 5.19, respectively. For this analysis we used the wavelength ranges $5600-6700 \mathrm{~cm}^{-1}$ and $7500-10000 \mathrm{~cm}^{-1}$. In both figures, the top panel shows the RV curves for the discharge cell (blue squares) and the absorption reference cell (red triangles). We subtracted the respective mean $\bar{v}_{\text {rad }}$ from the RV values to center the curves around zero. This procedure does not change the relative shift between the two sources. Moreover, the template spectra do not provide an absolute wavelength reference, because they are themselves prone to RV shifts. For completeness, we give the subtracted mean values: in Fig. $5.18,-0.11 \mathrm{~m} / \mathrm{s}$ for the $\mathrm{N}_{2}$ cell and $+0.94 \mathrm{~m} / \mathrm{s}$ for the $\mathrm{C}_{2} \mathrm{H}_{2}$ cell; in Fig. $5.19,-0.78 \mathrm{~m} / \mathrm{s}$ for the CN cell and $-0.16 \mathrm{~m} / \mathrm{s}$ for the $\mathrm{C}_{2} \mathrm{H}_{2}$ cell. The variations in the $\mathrm{RV}$ curves stay within $\pm 3 \mathrm{~m} / \mathrm{s}$ for almost all data points.

In the center panel, we present the difference between the RV data of the discharge cell and the absorption cell. This is the drift of the two spectra relative to each other. Assuming that the spectrum of the $\mathrm{C}_{2} \mathrm{H}_{2}$ cell is stable, we obtain the RV scatter of the discharge spectrum: the standard deviation of the data points is $1.0 \mathrm{~m} / \mathrm{s}$ and $0.8 \mathrm{~m} / \mathrm{s}$ for the $\mathrm{N}_{2}$ cell and $\mathrm{CN}$ cell, respectively. The measurement accuracy is limited by the $\mathrm{C}_{2} \mathrm{H}_{2}$ reference spectrum as discussed in the previous section. The errors on the individual data points of $\delta v_{\text {rad }}$ (in the center panels) are about $0.6-0.7 \mathrm{~m} / \mathrm{s}$. These error values are only about two times larger than the largest calculated uncertainties (for the $\mathrm{C}_{2} \mathrm{H}_{2}$ templates) as listed in Table 5.4. The agreement is even better if we take into account the cumulative value of the uncertainties from the two template spectra and the spectrum of the time series as listed in Table 5.4. We conclude that we measured the RV stability of the discharge lamp 
to an accuracy which is close to the theoretical limit based on the shape of the spectra and the noise in the observations.
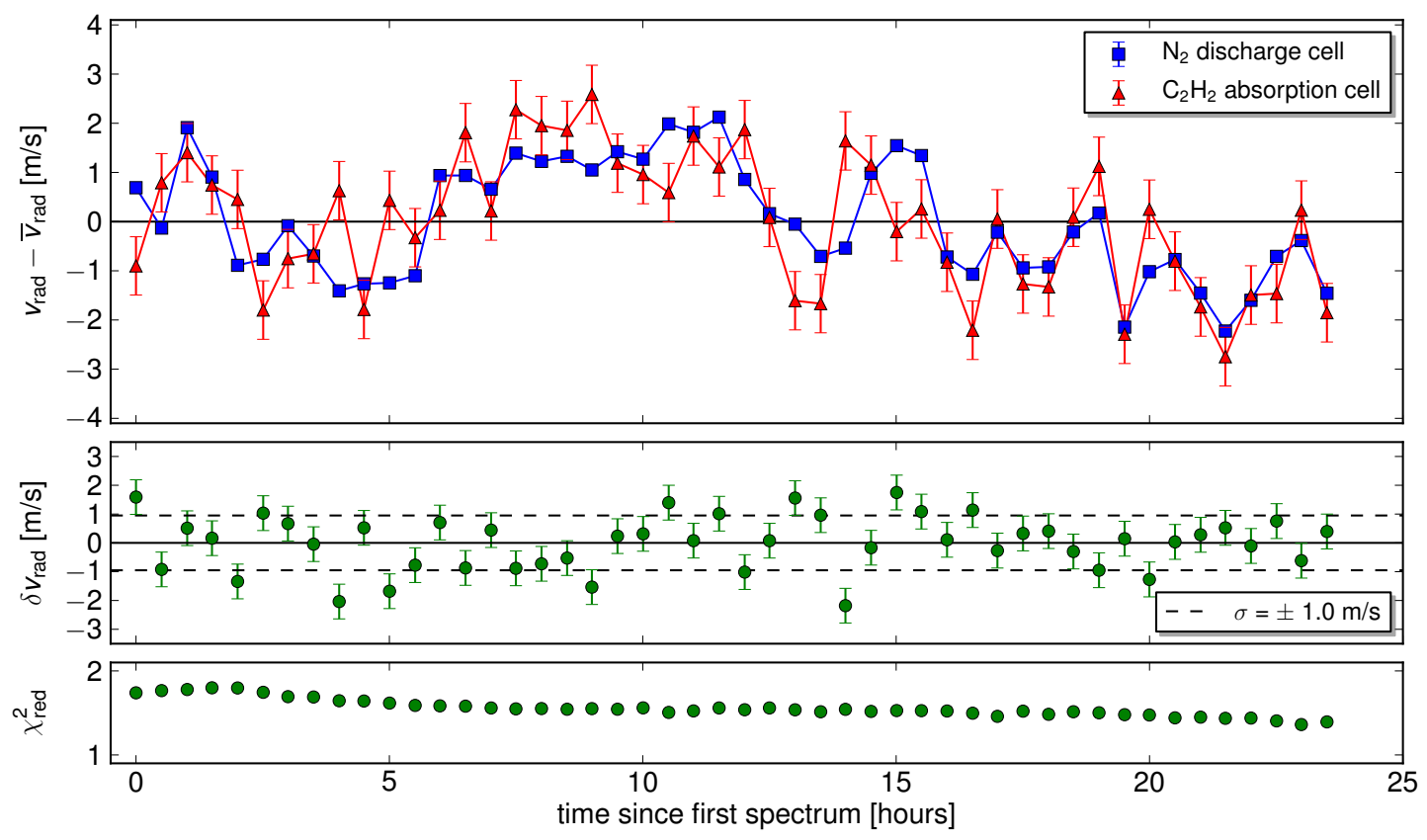

Figure 5.18: Radial velocity stability measurement for the spectrum of the $N_{2}$ discharge cell over one day. Top panel: The shifts of the template spectra for the discharge cell (blue squares) and for the reference gas cell (red triangles) required to match the spectra of the time series with the combined light from both sources. The respective mean is subtracted. Middle panel: The relative RV shift between the two sources. Lower panel: the reduced $\chi^{2}$ of the fitting process.

The bottom panel shows the reduced $\chi^{2}$ from the converged modeling process. For the $\mathrm{N}_{2}$ cell, the $\chi_{\text {red }}^{2}$ remains relatively constant between values 2 and 1 with a slight trend to lower values towards the end of the time series, i.e., closer to the recording time of the templates. We conclude that the spectrum is not subject to large variations that are not covered by the model (compare Eq. 5.3). The same curve for the CN cell shows a stronger increase in $\chi_{\text {red }}^{2}$ as we go further away in time from the template. Apparently, the spectrum of the CN cell undergoes larger changes than the spectrum of the $\mathrm{N}_{2}$ cell, and these changes cannot be accurately modeled. One effect that is not taken into account in the modeling is the different evolution of emission line intensities of different species. The spectrum of the $\mathrm{CN}$ cell contains emission features of $\mathrm{CN}, \mathrm{N}_{2}$ and other molecular and atomic byproducts, and we assume that all these species show slightly different intensity variations which lead to the increase in $\chi_{\text {red }}^{2}$. 

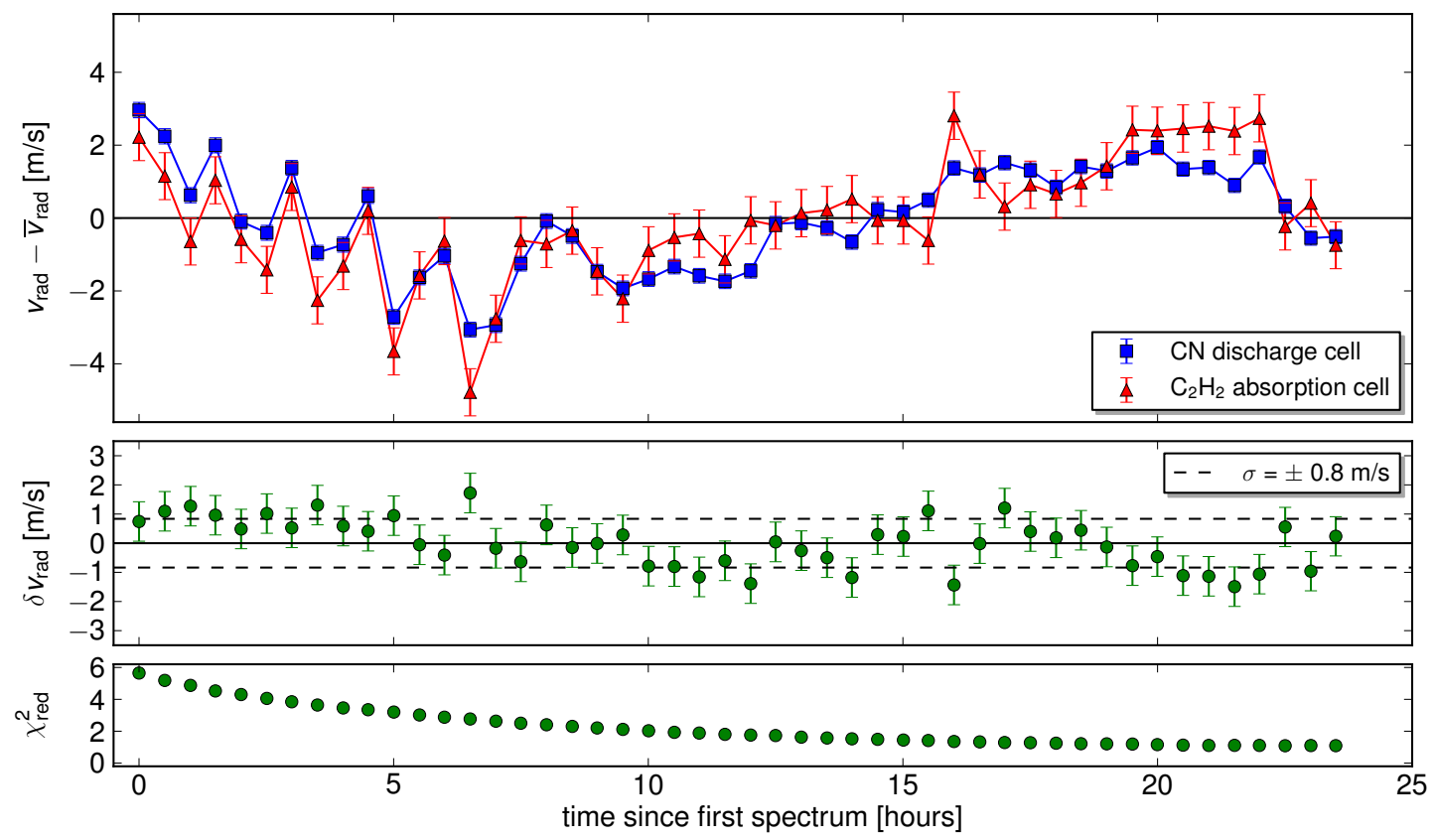

Figure 5.19: Same as Fig. 5.18, but for the CN discharge cell.

\subsubsection{The CN cell: spectral region of the $0-0$ band of $\mathrm{CN}$}

As we have seen from the histogram in Fig. 5.14, the spectrum of the CN cell is dominated by molecular nitrogen lines which amount for about $3 / 4$ of all detected lines. To get a better idea of the stability of the CN lines, we repeated the RV analysis with a smaller spectral range: we included only the wavenumber regions at $8700-9200 \mathrm{~cm}^{-1}$, which is dominated by the strong 0-0 vibrational band of CN (compare Fig. 5.3), and the region at $6454-6626 \mathrm{~cm}^{-1}$ for the $\mathrm{C}_{2} \mathrm{H}_{2}$ reference lines.

There is only a small change in the RV curve as compared to the analysis with the larger spectral range shown in Fig. 5.19 (relative RV scatter of $0.9 \mathrm{~m} / \mathrm{s}$ ). However, the trend in $\chi_{\text {red }}^{2}$ is much flatter, starting at around 2.4 and declining to 1 towards the end of the time series, similar to the $\chi_{\text {red }}^{2}$ trend for the measurements with the $\mathrm{N}_{2}$ cell shown in Fig. 5.18. This result supports the above mentioned conclusion that emission lines from different species exhibit a different variability in their line intensity over time. If we use a spectral region where one species dominates, the relative intensity changes are minimized and the modeling algorithm converges with a lower $\chi_{\text {red }}^{2}$. The line positions however appear to be equally stable independent of $\mathrm{CN}$ or molecular nitrogen lines. 


\subsection{Aging behavior 8}

Calibrators for astrophysical spectrographs need to maintain a stable spectrum over their anticipated operational lifetime. In practice, emission sources often show signs of aging. We conducted a dedicated observation run to study the behavior of the MIP lamp during longtime usage. We discuss the results of our measurements in comparison with information on HCLs, and also report previous aging experiences with the MIP lamp.

\subsubsection{Accelerated aging test}

We conducted an accelerated aging test with the $\mathrm{N}_{2}$ discharge cell. Before the aging test, the $\mathrm{N}_{2}$ discharge cell had been operated between September 2014 and June 2015 for about 105 hours in documented measurements which each lasted between 5 and 26 hours.

We started the aging test on 6 August 2015, keeping the lamp running continuously, and recording spectra twice a day. At each observation time, we recorded spectra of the $\mathrm{N}_{2}$ cell, the $\mathrm{C}_{2} \mathrm{H}_{2}$ cell and the combined light from both sources. We used the same experimental setup as for the RV measurements presented in Sect. 5.3. For the first six measurements (first 60 hours of operation), the MIP lamp operated with a stable reflected MW power of $\leq 1 \mathrm{~W}$. The reflected power, as measured at the MW generator, is the power not absorbed by the gas discharge, but instead reflected back to the generator. After about 72.75 hours, the reflected power increased above $15 \mathrm{~W}$ which triggered an automatic shutdown to prevent damage to the MW generator. Several attempts restarting the lamp were not successful. Although the gas inside the cell glowed when an active high frequency coil was positioned near the cell, a discharge was not sustained by the microwaves. The gas cell was removed from the experimental setup for inspection. No damage was visible at the gas cell or at any other part of the hardware.

We then heated the gas cell in an oven at $300^{\circ} \mathrm{C}$ for three hours and, afterward, could restart the discharge. The spectrum of the discharge recorded after the restart exhibited two obvious changes compared to the measurements that had been taken before the lamp shut down: the overall flux was lower and some strong lines appeared. Most of these new strong lines could be assigned to oxygen. The oxygen probably comes from the cell material (quartz glass) and was evaporated during the heating process. Approximately 10 hours after the restart, the lamp shut down again with the same characteristics as described before. We discuss this behavior in Sect. 5.4.2.

In Fig. 5.20, we show the RV curve for the accelerated aging test. We refer to Sect. 5.3.4.1 for a detailed description of the two panels. The RV shifts of the $\mathrm{N}_{2}$ discharge and the $\mathrm{C}_{2} \mathrm{H}_{2}$ absorption reference are measured relative to two templates that were recorded at the beginning of the time series. The first six data points represent the spectra that were recorded during the first 60 hours of the aging test. The lamp shut down at the time indicated by the dashed line (about 72.75 hours after the first spectrum). The last data

\footnotetext{
${ }^{8}$ This section has been submitted for publication to the scientific journal Astronomy $\mathcal{E}$ Astrophysics as Sect. 5 "Aging behavior" in "Near-infrared wavelength calibration with molecular discharge spectra of nitrogen and CN".
} 
point represents the measurement after heating the gas cell and restart of the discharge. The vertical dashed line at 113.5 hours indicates the second shut down. The stability of the line positions corresponds to an RV scatter of $2.2 \mathrm{~m} / \mathrm{s}$. It is noteworthy that the stability is preserved even after the heating process and the restart of the discharge.

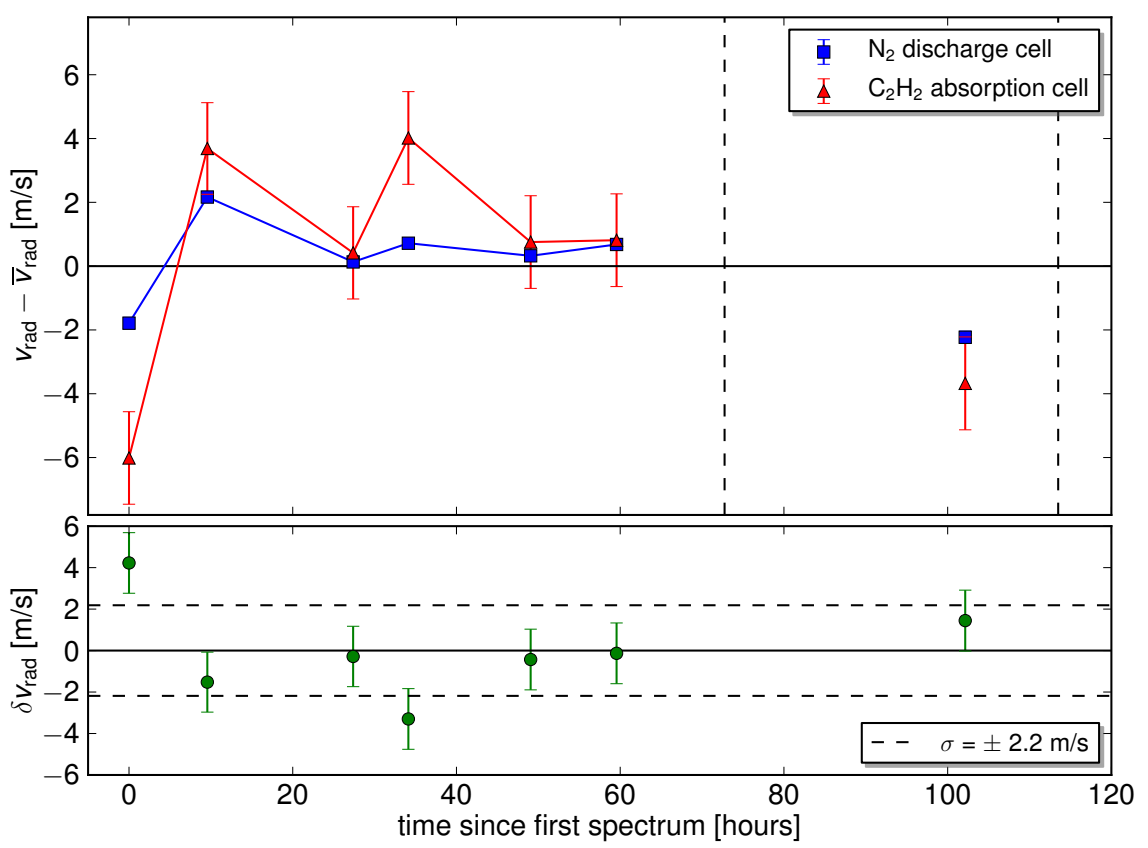

Figure 5.20: Radial velocity measurements during the accelerated aging test. Top panel: The shifts of the template spectra for the discharge cell (blue squares) and for the reference gas cell (red triangles) to match the spectra of the time series with the combined light from both sources. The respective mean is subtracted. The dashed vertical lines indicate the times when the lamp shut off. Lower panel: The relative RV shift of the two sources.

We also investigated the change in line intensities. In the spectrum of the nitrogen gas discharge, we can distinguish emission lines from atomic and molecular nitrogen. In Fig. 5.21, we show the evolution of the average line intensities for these two species. The values are calculated in the following way: in each of the seven recorded spectra, we determine the line intensities using the line fitting procedure described in Sect. 3.4.2 and the intensity of a line is divided by the intensity of the same line in the first spectrum. Then, we calculate the average intensity ratio for the lines from each species. Before the first shutdown of the lamp (indicated by the left dashed vertical line in Fig. 5.21), the molecular nitrogen lines remain at a nearly constant intensity. The intensities of the N I lines, however, increase linearly over time. In the last spectrum, lines from both species show intensities lower by a factor of 0.6 . This drop in intensity can also be influenced by a slightly different optical alignment of the discharge cell after its removal and reintegration into the experimental setup. We observe a larger scatter in the intensity for the molecular lines in the last spectrum, but there remains a clear difference between the data points from molecular and atomic lines. At this point, we can only speculate whether the change 
in the relative intensities between the two species is a symptom of aging, whether it is related to the cause of the lamp's shutdown, and whether it can be used to predict the remaining lifetime of the lamp.

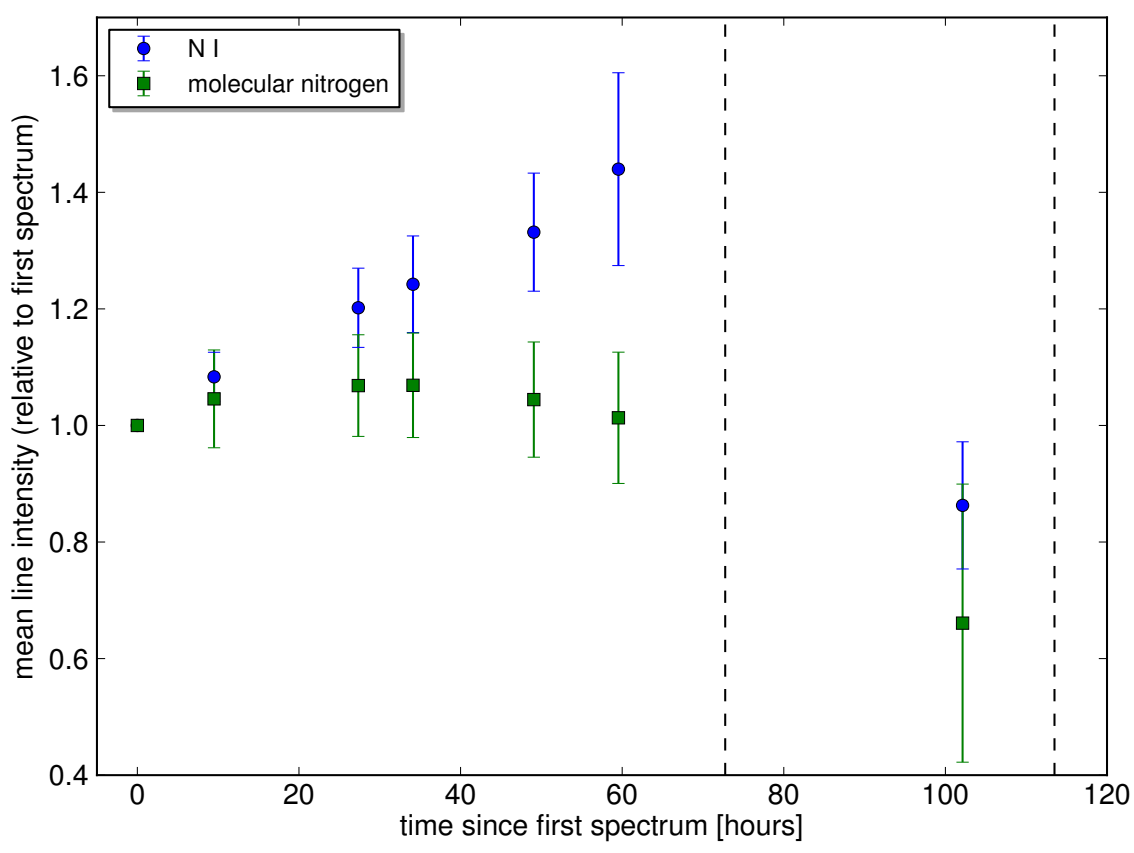

Figure 5.21: Evolution of mean line intensities of atomic and molecular nitrogen relative to the corresponding line intensities in the first spectrum. The dashed vertical lines indicate the times when the lamp shut off.

\subsubsection{Discussion of aging behavior}

A potential advantage of the MIP lamp is its electrodeless design. This mode of operation eliminates the sputtering process which is one source of aging in HCLs. Nevertheless, the MW powered discharge shut down after about 72.75 hours of continuous operation in the accelerated aging test. We believe that a different aging effect is observed in this test run, which has also been reported for HCLs: the so-called "clean-up" effect that describes the absorption of the gas by the surfaces inside the lamp (e.g., Kerber et al., 2007). In the electrodeless discharge lamp, the gas can be absorbed by the inner walls of the glass tube. This would lead to a decrease in gas pressure until the pressure is too low to sustain the discharge. If gas absorption by the glass enclosure at the plasma region is the limiting factor for the operation, a larger gas cell with a larger gas reservoir might extend the lifetime.

The heating of the gas cell, as described in the previous section, leads to outgassing of material from the glass. The evaporated material does not only include the original gas components, i.e., nitrogen molecules, but also particles from the glass tube. As the glass 
tube is made from quartz $\left(\mathrm{SiO}_{2}\right)$, this would explain the strong oxygen lines that are observed in the spectrum after the heating process. Lines from silicon could however not be seen in the spectrum. We expect that the resulting change in the gas composition, and consequently in the spectrum, will make the wavelength calibration less accurate, because the emission lines in a calibration spectrum do not anymore match prepared lines lists or a high-resolution spectral template.

For the aging test of the MIP lamp presented in the previous section, we decided not to switch the lamp on and off, but to keep the lamp running continuously. In contrast to HCLs, we argue that the ignition process should not have a strong influence on the aging behavior of the discharge lamp because of the electrodeless design. However, the uninterrupted operation could put a high amount of thermal stress on the material and therefore accelerate aging processes. If the lamp was operated for shorter times (e.g., 1 hour per night at an observatory), the operational time might increase significantly.

We have previously reported a similar behavior of atomic and molecular line intensities for a precursor of the CN discharge cell (Boesch et al., 2014). Additionally, we reported that the discharge of this discharge cell changed its color after a few days of continuous operation: the spectrum contained no molecular bands anymore, but solely atomic lines (Boesch et al., 2014). The fact that we observe a similar aging behavior with the $\mathrm{N}_{2}$ discharge cell, probably means that the aging process does not strongly depend on the gas mixture.

\subsection{Measurements with CRIRES}

The analyses of discharge spectra presented in the previous sections are based on observations with an FTS. However, grating spectrographs with CCD detectors are used almost exclusively for astronomical observations and every instrument has its own characteristics, e.g., regarding detector response. For this reason, wavelength calibration sources need to be tested with the instruments that are used for astronomical spectroscopy. Here, I present first measurements and wavelength calibration capabilities of the MIP lamp with the CRyogenic high-resolution InfraRed Echelle Spectrograph (CRIRES).

The CRIRES instrument has been operated at the Very Large Telescope (VLT) in Chile since the year 2006 (Käufl et al., 2004, Kerber et al., 2008). It provides a resolving power of up to 100000 in the spectral range from $1 \mu \mathrm{m}$ to $5 \mu \mathrm{m}$. In 2014/15, the instrument was disassembled and shipped to the headquarter of the European Southern Observatory (ESO) in Garching where it will be upgraded to become CRIRES+ (Follert et al., 2014). The instrument was reassembled in its original design and cooled-down to cryogenic temperatures for decommissioning. In this period, I recorded spectra of the MIP lamp with CRIRES on 6-8 October 2015 in Garching ${ }^{9}$. Due to the disassembly of the instrument in Chile and the subsequent reassembly in Germany, the wavelength calibration of CRIRES

${ }^{9}$ I thank the CRIRES consortium for the opportunity to carry out the measurements. I especially thank Ulf Seemann for his commitment and help during the observations and for extracting the spectra from the raw data. 
did not match the documented settings. This means that the recorded spectral range for a certain setting (grating and prism positions) was not known accurately. Only a few spectral ranges and instrument settings were calibrated with emission lines from a krypton lamp.

CRIRES is designed to operate at the Nasmyth focus of the telescope and the light enters the instrument through a dichroic window. The optical fiber that guides the light from the MIP lamp was connected to a collimating mirror (RC08SMA-P01 from Thorlabs) and positioned in front of the entrance window. The spectrum that is presented below was recorded with an exposure time of 10 seconds and 20 images were co-added. The MIP lamp was operated at a MW power of $50 \mathrm{~W}$. In addition, flat-field spectra with the halogen lamp (compare Sect. 5.2.1) and dark frames were recorded. Standard flat-field and dark corrections were applied during data reduction.

I show in Fig. 5.22 a spectrum of the CN discharge cell with emission lines from the 0-0 band of the Red System of CN. The blue curve represents a spectrum recorded with CRIRES covering the spectral range of one detector. The FTS spectrum of the same light source is shown in Fig. 5.22 as yellow curve. The latter has been convolved with a Gaussian kernel to broaden the spectral lines and to approximately match the resolving power of the grating spectrograph. Both spectra are normalized to the intensity of the strongest emission peak. The wavelength scale was assigned by matching the positions of emission lines in the CRIRES spectrum with the corresponding line positions from a spectrum recorded with the FTS as described below. Compared to the high-resolution FTS spectrum characterized in Sect. 5.2, the spectrum recorded with CRIRES contains more blended lines because of the lower spectral resolution.

For the wavelength calibration, I fitted emission lines in the CRIRES spectrum with Gaussian profiles to determine the line centers in pixel values. Next, peak positions in wavelengths were assigned using the spectral line list created with high-resolution FTS data of the same light source (see Sect. 5.2.1 and 5.2.3 for details on the FTS measurements). Special attention was given to blends in the CRIRES spectrum which shift the apparent positions of the corresponding lines. By comparison with the high-resolution FTS spectrum, the blended lines could be identified and avoided in the calibration process. Finally, 14 lines with a normalized intensity above 0.1 were selected to create the dispersion relation that is shown in the top panel of Fig. 5.23. A third order polynomial was fitted to the peak positions (green curve). The blue circles in the lower panel of Fig. 5.23 represent the residuals between the peak positions and the polynomial fit. The standard deviation in the residuals is about $100 \mathrm{~m} / \mathrm{s}$. For comparison, Nieva et al. (2011) report accuracy in the wavelength calibration of CRIRES of about $300 \mathrm{~m} / \mathrm{s}(1 / 10$ of a resolution element). They used the physical instrument model that is implemented in the CRIRES pipeline in combination with a model for telluric lines.

An additional method to determine the central lines positions was used to check the influence of broadened lines and possible effects of blends on the wavelength solution: The high-resolution FTS spectrum was convolved with a Gaussian kernel to broaden the spectral lines and to match the shape of the FTS spectrum with the CRIRES spectrum. Then, the positions of the same 14 emission lines were determined in the convolved FTS spectrum by fitting a Gaussian profile. These peak positions are compared with the dispersion 


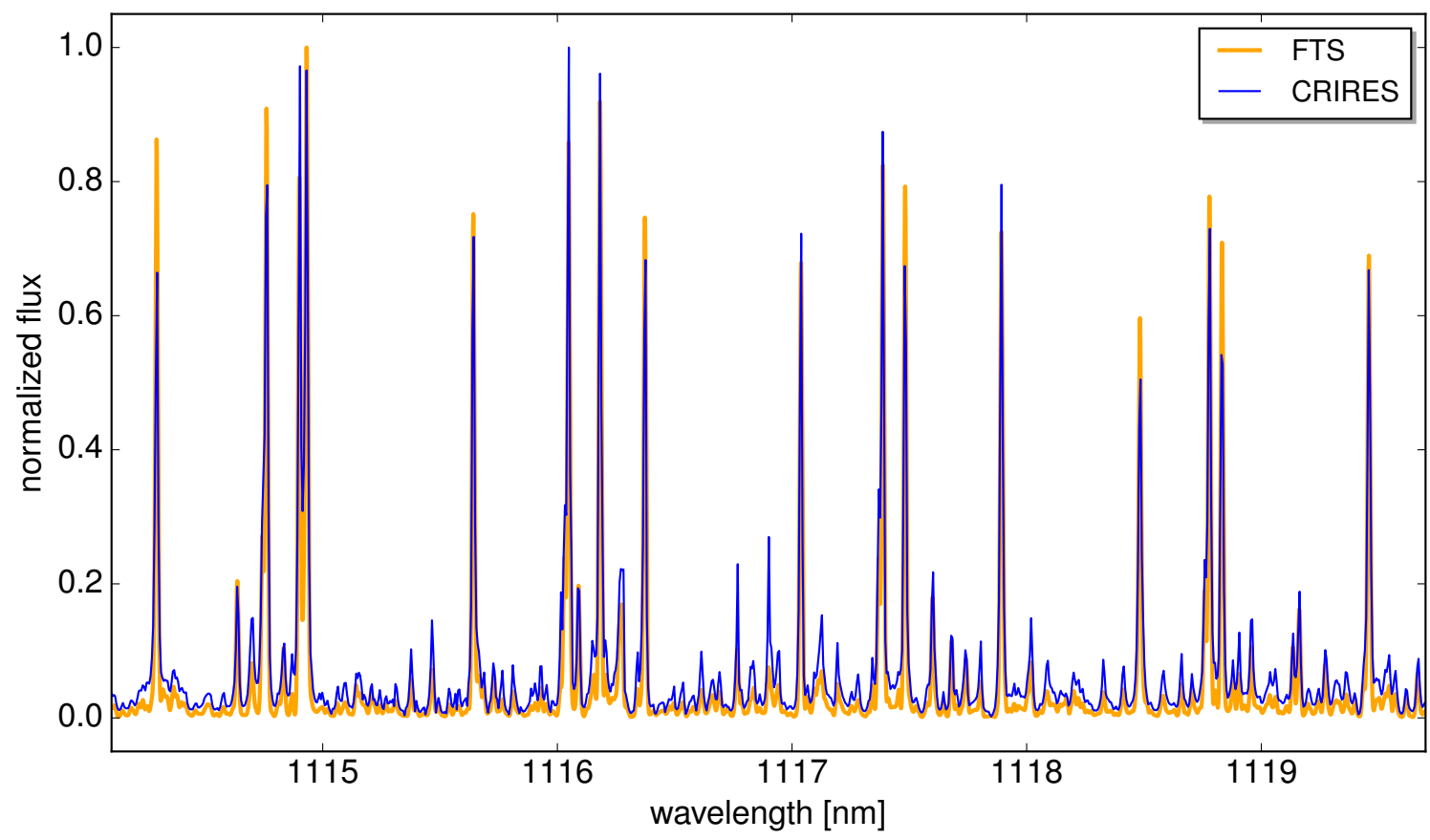

Figure 5.22: A wavelength calibrated spectrum recorded with the CRIRES spectrograph (blue curve). For comparison, an FTS spectrum of the same light source, degraded to a resolving power of $R=100000$, is shown in the background (yellow curve).

relation that has been determined using the peak positions from the original line list. The corresponding residuals are shown as orange triangles in the lower panel of Fig. 5.23. The scatter in the residuals is similar for both techniques. Consequently, the effect of line blends can be minimized if the emission lines, which are used for the wavelength calibration, are carefully selected. 


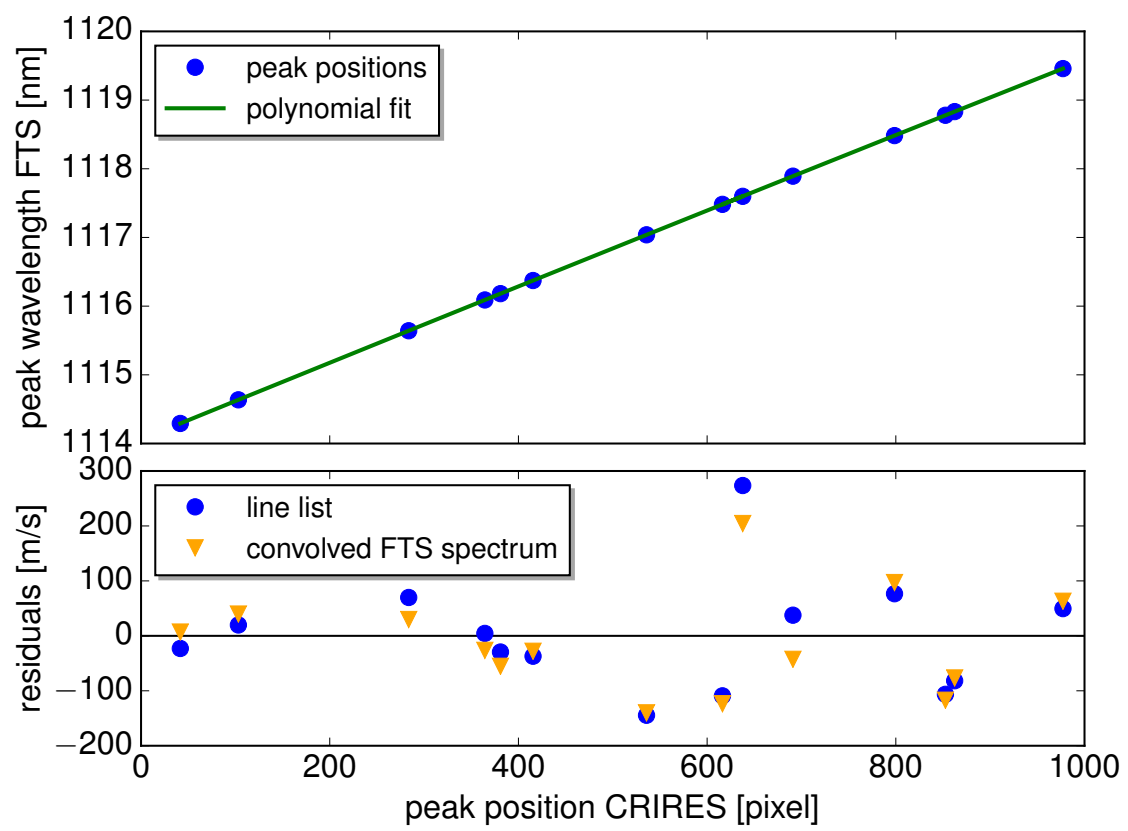

Figure 5.23: Top panel: dispersion relation for the detector using corresponding spectral line positions from a line list (in wavelengths) and in the CRIRES spectrum (pixel position). A 3rd order polynomial is fitted to the data points. Lower panel: residuals after the fit from the upper panel has been subtracted from the data points, using peak positions from the line list (blue circles) or peak positions determined from fits to broadened spectral lines (orange triangles; see main text for details). 


\title{
6 First tests with the discharge lamp in a magnetic field
}

\author{
Contents \\ 6.1 Introduction and motivation . . . . . . . . . . . 99 \\ 6.2 Selection and installation of permanent magnets $\ldots \ldots \ldots 101$ \\ 6.3 Calibration of the magnetic field with argon lines . . . . . . 104 \\ 6.4 Outlook . . . . . . . . . . . . . . . 108
}

\subsection{Introduction and motivation}

Application of the microwave-induced plasma (MIP) lamp in an astrophysical context is not limited to the task of wavelength calibration presented in Chap. 5. The lamp can be operated with discharge tubes filled with various gases. This allows the analysis of emission spectra of gaseous species that are of astrophysical interest. An application that I started to realize experimentally is the observation of spectra under the influence of a magnetic field. This chapter provides an outlook for future investigations by summarizing the modified experimental setup and first measurements.

Magnetic fields have been found in many stars across a wide range of spectral types. A review on magnetic fields in nondegenerate stars has been given by Donati \& Landstreet (2009). The main source of our knowledge of stellar magnetic fields is the atomic Zeeman effect (Illing, 1981). The Dutch physicist Pieter Zeeman first described the splitting of spectral lines under the influence of a magnetic field that can be directly observed through high-resolution spectroscopy (Zeeman, 1897). However, for dwarf stars of spectral type M and later, atomic lines decay rapidly and rotation-vibrational bands of molecules dominate the stellar spectra. Donati \& Landstreet (2009, Sect. 3.1) describe the resulting challenge in the following way: "The Zeeman effect of molecular lines, and in particular their Landé factor, is still rather poorly documented, with almost no measurements from laboratory experiments [...]." Laboratory measurements of molecular spectra with calibrated magnetic fields are therefore needed to derive Landé factors which characterize the strength of line splittings.

In the last years, attention has been given to the Wing-Ford system of iron hydride $(0-0$ band of the $\mathrm{F}^{4} \Delta-\mathrm{X}^{4} \Delta$ electronic system) as a diagnostic tool for stellar magnetic fields. Valenti et al. (2001) first observed Zeeman-broadened lines of iron hydride (FeH) in an M-dwarf, and compared them with magnetic sensitive lines of $\mathrm{FeH}$ in sunspot spectra 
published by Wallace et al. (1998). Shulyak et al. (2010) point out that modeling the Zeeman effect in $\mathrm{FeH}$ lines is complicated because the transitions follow intermediate Hund's cases and the theoretical description is based on certain approximations. Based on the formalism for the molecular Zeeman effect described in Berdyugina \& Solanki (2002), Shulyak et al. (2010) include FeH lines in the modeling of stellar spectra to confirm strong magnetic fields for the three M-dwarfs YZ CMi, EV Lac, and AD Leo. Improvements in modeling the molecular Zeeman effect are accompanied by new laboratory measurements: for example, scientists from a research group centered around the Institut Lumière Metière in Lyon use laser excitation spectroscopy to determine Landé factors of $\mathrm{FeH}$ (Crozet et al., 2012, 2014). Their experimental setup is described in detail in Vallon et al. (2009).

Beside $\mathrm{FeH}$, spectral lines of about ten other diatomic molecules have been observed in the visible part of sunspot spectra and in spectra of cool stars (Berdyugina \& Solanki, 2002). Among these species is the cyano radical (CN) that is investigated in the context of wavelength calibration of spectrographs in Chap. 5. Observations of CN lines at around $1100 \mathrm{~nm}$ in sunspot spectra were reported by Harvey (1973). He reports that the relative intensities of the Zeeman components of molecular lines are not necessarily symmetric in strong magnetic fields, and concludes that the molecular Zeeman effect is more complicated than the atomic Zeeman effect. In a follow-up study on Harvey's data, Illing (1981) writes that for certain molecules a fairly weak magnetic field can cause a Paschen-Back effect. In case of the $\mathrm{A}-\mathrm{X}$ transition of the $\mathrm{CN}$ molecule, the transition to the Paschen-Back regime can already occur for fields as weak as $77 \mathrm{G}$ (Berdyugina \& Solanki, 2002). Berdyugina \& Solanki (2002) claim that the Red System of CN would be a sensitive tool for solar and stellar magnetic studies if the Paschen-Back effect is properly included. It should be investigated if laboratory emission spectra of CN can be recorded with the MIP lamp under the influence of a magnetic field to study the behavior of the spectral lines and to determine Landé factors.

The characterization of magnetic fields in cool stars is important for the general understanding of their generation and evolution (Shulyak et al., 2010). Furthermore, stellar activity is believed to be a by-product of magnetic fields generated within the convective envelope of cool stars through dynamo processes (Donati \& Landstreet, 2009). This has an impact on radial velocity observations because activity phenomena, such as stellar spots, are limiting the accuracy of these measurements (e.g., Perryman, 2011). A good understanding of activity processes is therefore necessary to disentangle stellar activity and exoplanet signatures in radial velocity measurements.

In this chapter, I present the initial progress of installing permanent magnets in the experimental setup and determining the magnetic field strength. The selection of magnets and the design of the modified experimental setup is described in Sect. 6.2. A first measurement of the magnetic field strength using the Zeeman-splitting of argon lines is presented in Sect. 6.3. Finally, an outlook for future work is given Sect. 6.4. 


\subsection{Selection and installation of permanent magnets}

In this section, I explain the modifications that have been made to the experimental setup. See Chap. 4 for a description of the setup as a whole. The objective was to install a device creating a magnetic field that is homogeneous at the point where the light is emitted, and that is strong enough to induce detectable Zeeman-splitting. The limited space around the microwave cavity, where the light is emitted, turned out to be a main challenge and eliminated many design options. In addition, the gas discharge cannot be approximated as a point source, but extends a few centimeters inside (and even a little bit outside) the MW cavity. This makes it more complicated to create a homogeneous field over the whole region of light emission.

Following the approach presented in Vallon et al. (2009), I decided to use permanent magnets to create the magnetic field. Permanent magnets made from an alloy of neodymium, iron and boron ( $\mathrm{NdFeB}$, neodymium magnet) are the strongest type of permanent magnets commercially available (Fraden, 2010, Sect. 3.3.4). Crozet et al. (2012, 2014) report that they created a field of up to $0.5 \mathrm{~T}$ using $\mathrm{NdFeB}$ magnets and a ferromagnetic circuit. To observe a clear separation of the $\sigma$-components even under the influence of a weaker magnetic field, I decided to position the magnets in such a configuration that the magnetic field lines follow the direction of the optical axis. This suppresses the central Zeemancomponent ( $\pi$-component) in the observed spectrum (longitudinal Zeeman effect). See, e.g., Haken \& Wolf (2004) for a full description of the Zeeman effect.

The program FEMM was used to simulate different types and arrangements of NdFeB magnets, and to estimate the achievable magnetic field strength. FEMM (Finite Element Method Magnetics) is a program for solving electromagnetic problems in two dimensions by finite element analysis ${ }^{1}$. According to the program's manual, an NdFeB magnet can be modeled based on the knowledge of two quantities: the coercivity and the permeability. The coercivity $H_{\mathrm{c}}$ is the absolute strength of an external magnetic field that is needed to demagnetize the magnet. It can be calculated from the maximum magnetic energy $E$. This is the maximum value of the product $B \cdot H$ given by the Demagnetization Curve (BH curve) of the magnet. The grade of the magnet is derived from this quantity, e.g., grade N42 for a magnet with $E=42$ MGOe (MGOe: Mega Gauss Oersted). The manual from FEMM states the following relation between the coercivity and the maximum magnetic energy (see also Campbell, 1994):

$$
H_{\mathrm{c}} \approx \frac{5 \cdot 10^{5} \sqrt{E[\mathrm{MGOe}]}}{\pi} \frac{\mathrm{A}}{\mathrm{m}} .
$$

This results in $H_{\mathrm{c}}=1031442 \mathrm{~A} / \mathrm{m}$ for $E=42$ MGOe. A good assumption for the relative permeability is $\mu_{\mathrm{r}}=1$, according the manual of the FEMM software.

In Fig. 6.1, I show the absolute magnetic flux density $|B|$ for configurations with two different pairs of magnets as simulated with FEMM. Both panels show two-dimensional maps of a plane containing the optical axis. The optical axis is the rotational axis of

\footnotetext{
${ }^{1}$ http://www.femm.info, Version 4.2
} 


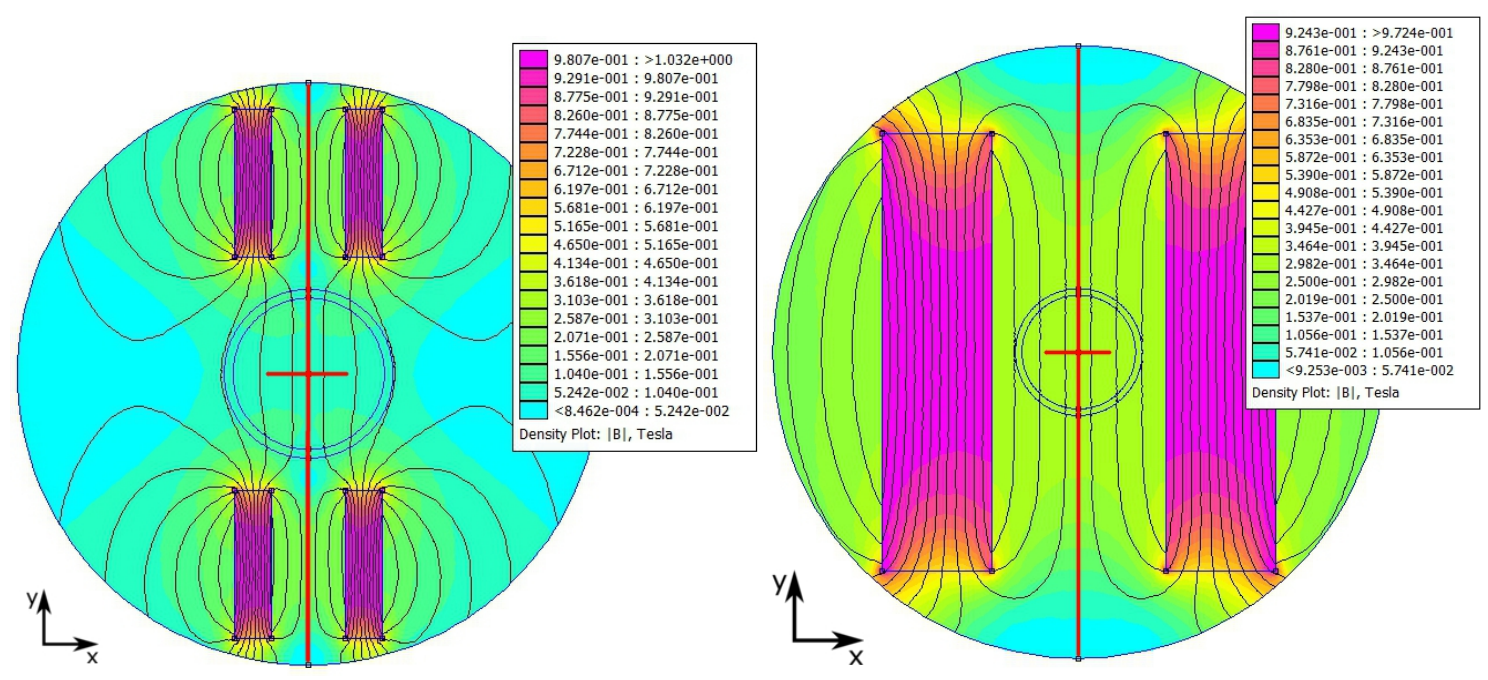

Figure 6.1: Maps of the magnetic flux density as simulated with FEMM in a plane containing the optical axis (red vertical line). The circles in the middle mark the inner and outer diameters of the cavity. Left panel: Two ring magnets with outer diameter of 1 inch, inner diameter of 0.5 inch and length of 1 inch (represented by bar magnets in this two-dimensional simulation). Right panel: Two block magnets with a length of $100 \mathrm{~mm}$ and a width of $25 \mathrm{~mm}$.

the discharge gas cell and it extends along the central vertical axes of the two maps (red vertical line). For the left panel, I simulated two ring magnets with an outer diameter of 1 inch, an inner diameter of $0.5 \mathrm{inch}$ and a length of $1 \mathrm{inch}$. The grade of the NdFeB magnets is N42. In a plane, one ring magnet can be represented by two bar magnets. The ring magnets are aligned along the optical axis in such a way that the discharge gas cell fits through the holes of the ring magnets and the MW cavity (refer also to Fig. 6.3 for orientation). The size of the MW cavity is also shown in Fig. 6.1 as circles for orientation, with an inner diameter of $26 \mathrm{~mm}$ and an outer diameter of $29 \mathrm{~mm}$. We see in the left panel, that the magnetic field lines are close to parallel in the range of the cavity and inside the ring magnets. However, the field changes orientation along the optic axis between the cavity and the magnets, and this causes a strong variation in the absolute magnetic flux density.

The right panel shows an alternative configuration with two block magnets that are arranged alongside the gas cell. The magnets have a length of $100 \mathrm{~mm}$ and a width of $25 \mathrm{~mm}$. The space around the MW cavity would allow for a height of $10 \mathrm{~mm}$, but this is not represented in the two-dimensional simulation. A stronger and more homogeneous field seems to be possible with these magnets compared to the configuration with ring magnets.

Figure 6.2 shows cuts through the magnetic field along two different spatial axes as calculated from the two simulations shown in Fig. 6.1. The left panel shows the tangential field along the axis of the gas cell, i.e., the value of the $y$-component of the magnetic field vector along the red vertical line in Fig. 6.1. The blue curve represents the case with the ring magnets and the green curve represents the case with the block magnets. We can see 
clearly the variation in the field created by the ring magnets. However, in the proximity of the MW cavity, marked by the dashed lines, the field strength is stable within a range of about $0.02 \mathrm{~T}$ with a maximum of about $0.11 \mathrm{~T}$. The block magnets create a field with a maximum of about $0.32 \mathrm{~T}$ at the center of the MW cavity. The field strength drops below $20 \%$ of the peak value not before a distance of about $4 \mathrm{~cm}$ from the cavity's center. In the right panel, I show the magnetic flux density in $y$-direction across the diameter of the gas cell (indicated by the red horizontal line in Fig. 6.1). The trend is approximately constant for both configurations. Note that the dashed lines in the right panel mark the size of the gas cell and not of the MW cavity.

I also investigated the normal field along the optical axis and the tangential field along the diameter of the gas cell. The field strengths in these directions are negligibly small.
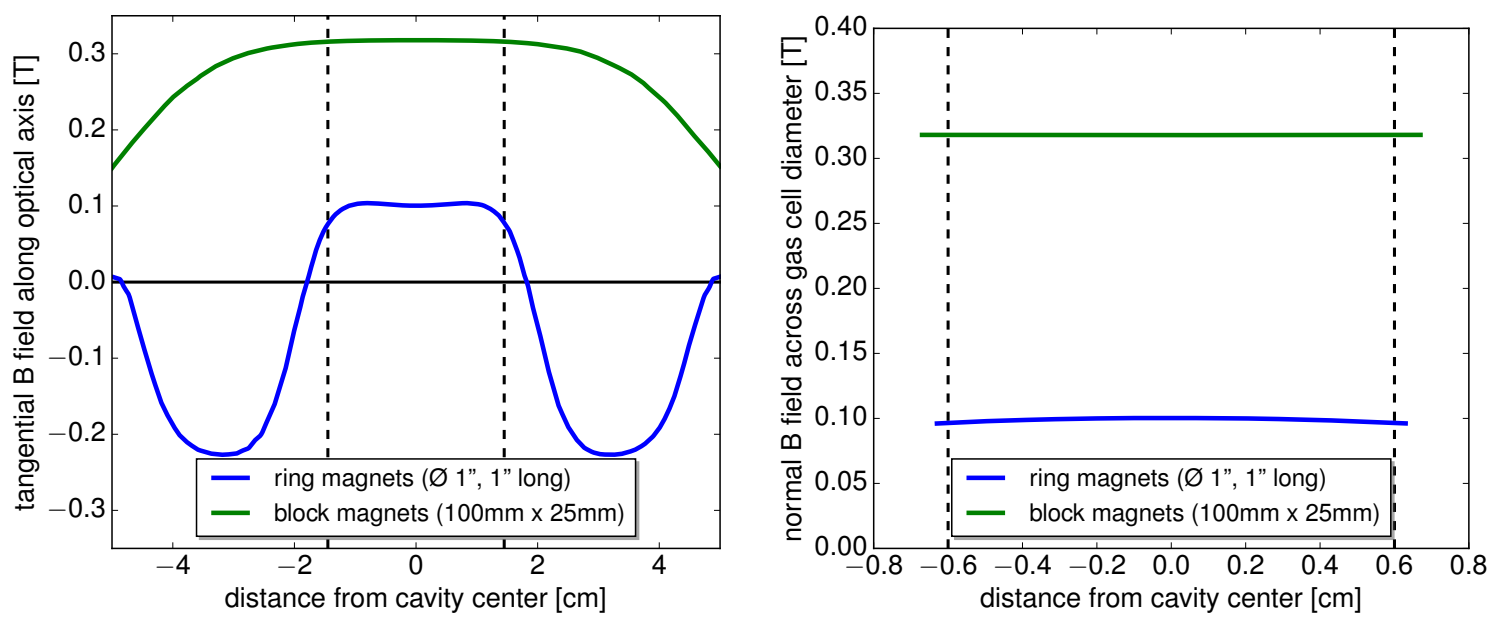

Figure 6.2: Calculated magnetic flux density for configurations with ring or block magnets. Left panel: The tangential component of the magnetic field along the optical axis (along the axis of the gas cell). The black vertical lines indicate the outer diameter of the MW cavity. Right panel: The normal component of the magnetic field along the diameter of the gas cell. The black vertical lines mark the diameter of the gas cell.

As already mentioned above, the available space in the experimental setup limits the options for the size and the shape of the magnets. This is mainly due to the connectors for the coaxial cable (MW power supply) and for the air hose (cooling) at the MW cavity. Both connectors are positioned at the intersection of the MW cavity and the gas cell (see Figs. 4.3 and 4.6 in Chap. 4 for a picture of the experimental setup and a larger picture of the MW cavity, respectively). Another restriction is the limited choice of shapes and sizes for the magnets that are commercially and off-the-shelf available. I could not find the type of block magnet that has been discussed above in the inventory of any supplier of NdFeB magnets. The usual magnetization direction for commercially distributed magnets appears to be along the shortest edge of a magnet, and not along the longest edge as used for the simulation shown in the right panel of Fig. 6.1.

Therefore, I use ring magnets with the above-mentioned dimensions (model RX08X0 from K\&J Magnetics). These magnets have the practical advantage that they can be held by a 
standard cage plate (Thorlabs CP02/M) with an inner bore of 1 inch and can be easily integrated into the existing cage system. A schematic drawing of the modified experimental setup is shown in Fig. 6.3.
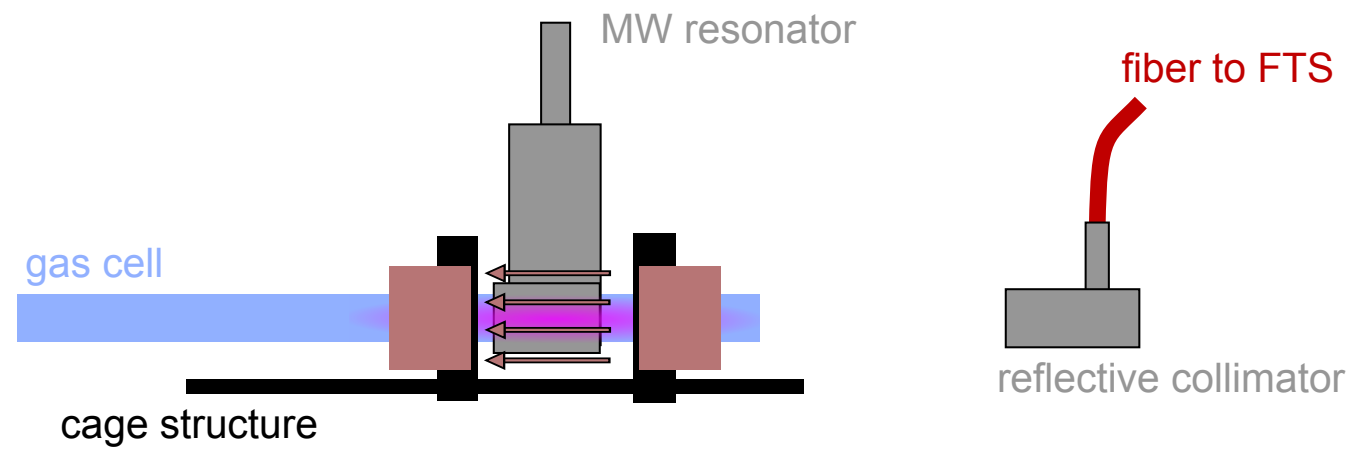

$(\mathrm{N})$ ring magnets $(\mathrm{S})$

Figure 6.3: Simplified drawing of the modified experimental setup (view from the side). One ring magnet is installed on each side of the microwave cavity along the axis of the discharge gas cell. The magnets are oriented in the same direction (the arrows indicate the direction of the magnetic field for the shown configuration). Not all optical elements and support structures are shown.

\subsection{Calibration of the magnetic field with argon lines}

Before the experimental setup can be used to determine unknown parameters of molecular transitions, the magnetic field needs to be calibrated. This can be done by measuring the Zeeman-splitting of spectral lines that have known Landé factors. Following the approach by Crozet et al. (2014), I use three transitions of neutral argon. The reference data for these transitions is listed in Table 6.1. The transition wavenumbers $\tilde{\nu}_{0}$ and the corresponding energy states ( $E^{\prime \prime}$ and $E^{\prime}$ for the lower and upper state, respectively) can be found in the "Lines" section of the NIST Atomic Spectra Database (Kramida et al., 2014). The quantum numbers $J$ and Landé factors $g$ are listed in the "Levels" section of the same database. The last three columns of Table 6.1 list the expected number of Zeeman-components for each line. For example, the spectral line at $10351.5012 \mathrm{~cm}^{-1}$ splits into two $\pi$-components, two $\sigma^{-}$-components, and two $\sigma^{+}$-components; the full Zeeman-pattern consists of six lines.

\begin{tabular}{c|cc|cc|cc|ccc}
\hline \hline$\tilde{\nu}_{0}\left[\mathrm{~cm}^{-1}\right]$ & $E^{\prime \prime}\left[\mathrm{cm}^{-1}\right]$ & $E^{\prime}\left[\mathrm{cm}^{-1}\right]$ & $J^{\prime \prime}$ & $J^{\prime}$ & $g^{\prime \prime}$ & $g^{\prime}$ & $\sigma^{-}$ & $\pi$ & $\sigma^{+}$ \\
\hline 10351.5012 & 93750.5978 & 104102.0990 & 1 & 1 & 1.404 & 1.985 & 2 & 2 & 2 \\
10837.7242 & 95399.8276 & 106237.5518 & 1 & 2 & 1.102 & 1.305 & 3 & 3 & 3 \\
10958.3390 & 93143.7600 & 104102.0990 & 2 & 1 & 1.506 & 1.985 & 3 & 3 & 3 \\
\hline
\end{tabular}

Table 6.1: Reference data for the three Ar I lines that are used to calibrate the magnetic field. Data from NIST (Kramida et al., 2014). The last three columns give the number of Zeeman-components in the different polarization directions.

Spectra were recorded using a sealed discharge cell filled with 2 mbar of argon. The MW 
discharge lamp was operated at a power of $50 \mathrm{~W}$, and the FTS was set to a resolution of $\Delta \tilde{\nu}_{\text {OPUS }}=0.03 \mathrm{~cm}^{-1}$, an aperture diameter of $1.3 \mathrm{~mm}$, CaF beamsplitter, and InSb detector. The spectra were recorded on 30 June 2015.

In order to describe the line splitting of argon with the Zeeman effect, the magnetic field must be "weak". According to Haken \& Wolf (2004, Sect. 13.4), a "weak" magnetic field for a certain atom means that the splitting of the energy levels caused by the magnetic field is small compared to the fine structure splitting. The strength of spin-orbit coupling increases with the atomic number and a "strong" field is realized at lower magnetic field strengths for lighter atoms as compared to heavier ones. For sodium (with atomic number $Z=11$ ) a magnetic field with $B=3 \mathrm{~T}$ is still "weak" (Haken \& Wolf, 2004). Therefore, the expected magnetic flux density of about $0.1 \mathrm{~T}$ (see Fig. 6.2) can be considered as "weak" for $\operatorname{argon}(Z=18)$, and the line splitting can be described by the Zeeman effect.

The additional energy for each Zeeman-splitted energy level gained from the external magnetic field $B$ is given by:

$$
\Delta E=m_{J} \cdot g \cdot \mu_{\mathrm{B}} \cdot B
$$

where $m_{J}$ is the magnetic quantum number $m_{J}=J, J-1, \ldots,-J$ and $\mu_{B}=e \hbar / 2 m_{\mathrm{e}}$ is the Bohr magneton $\left(\hbar=h / 2 \pi\right.$ : reduced Planck constant, $e$ : elementary charge, $m_{\mathrm{e}}$ : electron rest mass; Haken \& Wolf, 2004, Sect. 13.3). Thus, the separation in wavenumber for a transition between two Zeeman-splitted energy levels with quantum numbers $m_{J^{\prime}}$ and $m_{J^{\prime \prime}}$ is

$$
\begin{aligned}
\Delta \tilde{\nu} & =\left(\Delta E_{m_{J^{\prime}}}-\Delta E_{m_{J^{\prime \prime}}}\right) /(h c) \\
& =\left(m_{J^{\prime}} \cdot g^{\prime} \cdot \mu_{\mathrm{B}} \cdot B-m_{J^{\prime \prime}} \cdot g^{\prime \prime} \cdot \mu_{\mathrm{B}} \cdot B\right) /(h c) \\
& =\left(m_{J^{\prime}} \cdot g^{\prime}-m_{J^{\prime \prime}} \cdot g^{\prime \prime}\right) \mu_{\mathrm{B}}^{*} B,
\end{aligned}
$$

where I introduced $\mu_{\mathrm{B}}^{*}=\mu_{\mathrm{B}} /(h c) \approx 0.46686(\mathrm{~T} \cdot \mathrm{cm})^{-1}$. Not all Zeeman-components are resolved if the magnetic field is too weak. Instead, the different lines of the $\sigma$-components overlap. In this case, the so-called effective Landé-factor $g^{*}$ can be used to calculate the wavelength separation of the center of gravity of the $\sigma$-components with respect to the line center at $\tilde{\nu}_{0}$ (Landi Degl'Innocenti, 1982):

$$
\begin{aligned}
g^{*} & =\frac{1}{2}\left(g^{\prime}+g^{\prime \prime}\right)+\frac{1}{4}\left(J^{\prime}-J^{\prime \prime}\right)\left(g^{\prime}-g^{\prime \prime}\right)\left(J^{\prime}+J^{\prime \prime}+1\right) \\
& =\frac{1}{2}\left(g^{\prime}+g^{\prime \prime}\right)+\frac{1}{4}\left(g^{\prime}-g^{\prime \prime}\right)\left(J^{\prime}\left(J^{\prime}+1\right)-J^{\prime \prime}\left(J^{\prime \prime}+1\right)\right) .
\end{aligned}
$$

Substituting the effective Landé factor into Eq. 6.1 for the term $\left(m_{J^{\prime}} \cdot g^{\prime}-m_{J^{\prime \prime}} \cdot g^{\prime \prime}\right)$, the positions of the unresolved $\sigma$-components are calculated:

$$
\tilde{\nu}_{\sigma}=\tilde{\nu}_{0} \pm \Delta \tilde{\nu}=\tilde{\nu}_{0} \pm g^{*} \cdot \mu_{\mathrm{B}}^{*} \cdot B
$$

In Figure 6.4, I show the three argon lines observed without a magnetic field (blue crosses) and with the configuration of magnets shown in Fig. 6.3 (green circles). It can be seen that 
the argon lines split into two $\sigma$-components and that the central $\pi$-component is strongly suppressed as expected. I fitted a Gaussian profile to the unsplitted line (blue curve) to determine the exact line center, line width, and line intensity. Then, I fitted a superposition of three Gaussian profiles to the splitted line. One Gaussian function is centered at the position of the unsplitted line, while the other two lines are centered at $\tilde{\nu}_{\sigma}$ as given by Eq. 6.3. The fit parameters are the magnetic flux density $B$, the intensities, and the width of the broadened profiles. In the middle panel of Fig. $6.4\left(\tilde{\nu}_{0}=10937.7242 \mathrm{~cm}^{-1}\right)$, a small peak is visible at around $\tilde{\nu}_{0}$ in the case with magnetic field. This is probably a weak emission line from a different species, most likely due to residual air inside the discharge gas cell.

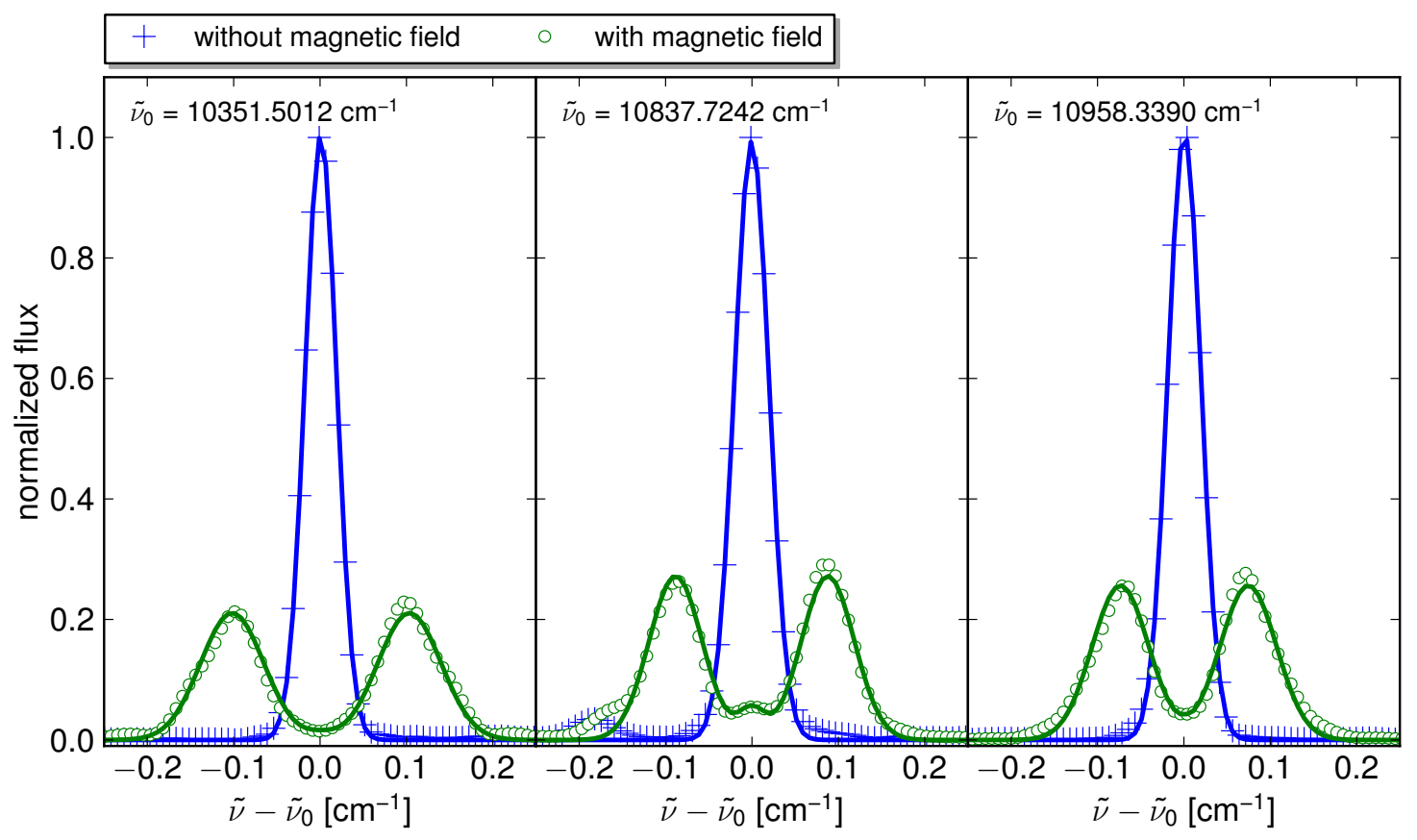

Figure 6.4: Spectral lines of neutral argon for the transitions listed in Table 6.1. Experimental data is shown as blue crosses (without magnetic field) and green circles (with magnetic field). The solid curves in the respective colors are fits to the data.

\begin{tabular}{ccc}
\hline \hline Transition $\tilde{\nu}_{0}\left[\mathrm{~cm}^{-1}\right]$ & $g^{*}$ & $B[\mathrm{~T}]$ \\
\hline 10351.5012 & 1.695 & 0.130 \\
10837.7242 & 1.407 & 0.134 \\
10958.3390 & 1.267 & 0.125 \\
\hline
\end{tabular}

Table 6.2: Effective Landé factors for the three argon transitions, and the magnetic field as calculated from the Zeeman-splitted line patterns.

Table 6.2 lists the effective Landé factors of the three argon transitions as calculated with Eq. 6.2 using the data from Table 6.1. Furthermore, it lists the fit results for the magnetic flux density $B$. The error on $B$, as calculated from the weighted fit, is about $10^{-5} \mathrm{~T}$. 
However, the systematic error can be expected to be larger considering the spread in $B$ for the three transitions. Thus, I rounded the results to the first decimal place where the results differ. The best determination of the magnetic flux density using the three values from Table 6.2 is

$$
B=\bar{B} \pm \frac{t \cdot s}{\sqrt{N}}=(0.130 \pm 0.003) \mathrm{T}
$$

where $\bar{B}$ is the mean and $t=1.32$ is the Student-t-factor for $N=3$ measurements (Drosg, 2009). The standard deviation of the single measurements is given by $s$. This value for $B$ is about $20 \%$ higher than the maximum of the simulated field inside the MW cavity of about $0.11 \mathrm{~T}$ (compare Fig. 6.2). The simulated value should be treated rather as an estimate and not as a reference. Considering the approximations in the theoretical treatment and the uncertainties in the magnets' properties, the agreement between the experimental and calculated values is satisfactory.

I also tried to fit the magnetic data shown in Fig. 6.4 with a superposition of Gaussian profiles for the full Zeeman-pattern with separations in wavenumber as described by Eq. 6.1. However, this method does not properly converge or does not give consistent results for the magnetic field because the lines are not resolved.

A more precise determination of the magnetic field should be possible if all Zeemancomponents are resolved. Therefore, I estimate the minimum magnetic field that would cause a sufficiently large line splitting. Figure 6.5 shows a superposition of Gaussian profiles simulating the Zeeman-pattern of the transition centered at $\tilde{\nu}_{0}=10837.7242 \mathrm{~cm}^{-1}$ for different magnetic flux densities and line widths. Note that only the $\sigma$-components are plotted. The line width is calculated from pure Doppler broadening at a temperature $T$. For orientation, the left panel of Fig. 6.5 shows the case with parameters that correspond to the measurements presented above in this section $(B=0.13 \mathrm{~T}, T=800 \mathrm{~K})$. The temperature is a rough estimate derived from the line widths of the three observed unsplitted argon lines. For the graph in the middle panel, I kept the temperature constant, but increased the value for $B$. For a line width that corresponds to a temperature of $T=800 \mathrm{~K}$, the magnetic flux density needs to be increased to about $B=0.34 \mathrm{~T}$ to resolve the three $\sigma^{-}$ and three $\sigma^{+}$lines. We can compare this value for $B$ with the maximum magnetic field created with the block magnets that have been discussed in Sect. 6.2 as an alternative to the ring magnets. The field created with the block magnets reaches a maximum flux density of $0.32 \mathrm{~T}$ and the line splitting would probably not be visible. However, the line width can be decreased if the lamp is operated at a lower MW input power, e.g., using $25 \mathrm{~W}$ instead of $50 \mathrm{~W}$. With a reduced line width, there is a good chance of resolving the full Zeeman-pattern with a magnetic flux density of $B=0.32 \mathrm{~T}$.

I carried out the same kind of simulations for the other two argon lines: the transition centered at $10958.3390 \mathrm{~cm}^{-1}$ is already resolved for weaker fields $(B \approx 0.15 \mathrm{~T})$; the transition centered at $10351.5012 \mathrm{~cm}^{-1}$ theoretically requires a magnetic field of $B \approx 0.12 \mathrm{~T}$ to be resolved. The instrumental broadening of the overlapping lines probably prevents to see the individual line peaks of the latter transition in Fig. 6.4. In the right panel of Fig. 6.5, I show a fully resolved Zeeman-pattern for comparison. The values for $B$ and $T$ in the right panel are similar to the conditions of the experiments conducted at the Institut Lumière 
Metière in Lyon (P. Crozet, private communication). With the fully resolved set of lines, the research group in Lyon is able to determine the magnetic field with an accuracy of 0.5\% (Crozet et al., 2012, 2014).

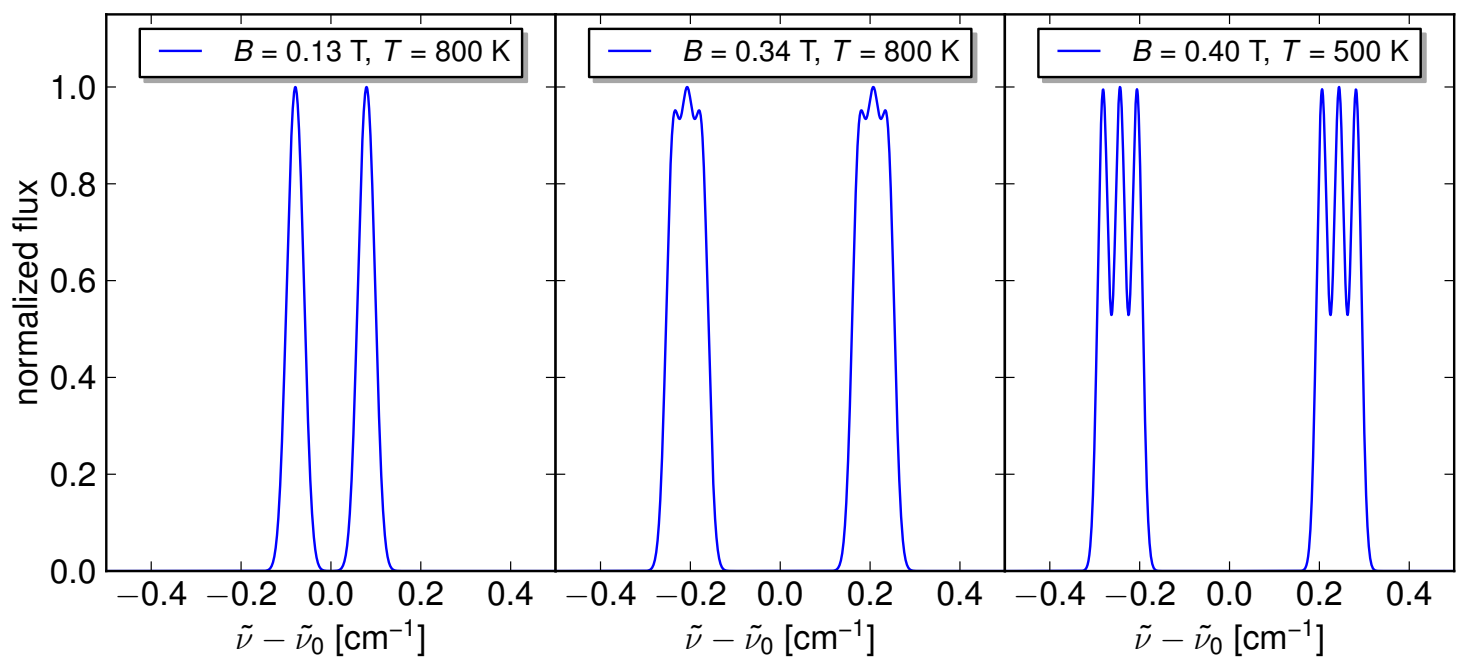

Figure 6.5: Simulated Gaussian-profiles illustrating the expected Zeeman-splitting of the argon line at $\tilde{\nu}_{0}=10837.7242 \mathrm{~cm}^{-1}$. Line separation is calculated based on the magnetic flux density $B$ and the Landé factors given in Table 6.1. The line width is calculated from the temperature $T$ assuming pure Doppler broadening.

\subsection{Outlook}

In the previous sections, I presented the results from the first tests with the discharge lamp in a magnetic field. With the current experimental setup, a magnetic field with a flux density of $B=0.13 \mathrm{~T}$ can be created. The flux density is measured with an accuracy of about $3 \%$ from the Zeeman-splitting of three argon transitions. A more accurate determination of the magnetic field should be possible if all Zeeman-components are resolved using a stronger magnetic field (compare results from Crozet et al., 2014). Therefore, future work on the experimental setup should aim to increase the magnetic field strength. At the same time, the homogeneity of the field should be increased. The equipment to achieve these goals might include the utilization of a ferromagnetic circuit, custom made $\mathrm{NdFeB}$ magnets or magnetic coils. An experimental setup with a well-calibrated magnetic field is a requirement for any further scientific studies.

Once the calibration is accurate enough, a scientific focus could be the study of molecular spectra under the influence of a magnetic field. As mentioned in the introduction (Sect. 6.1), about ten different diatomic molecules, including $\mathrm{CN}$, have been identified in sunspot spectra. They could serve as tools to study the magnetic fields in cool stars. This requires a good understanding of the magnetic sensitivity of the individual spectral lines, and of the underlying theoretical mechanism. Laboratory measurements are needed to identity 
spectral lines that show a high sensitivity for magnetic fields and can be observed in stellar spectra.

First test measurements of the CN spectrum under the influence of a magnetic field have been carried out. Fig. 6.6 shows the spectrum of $\mathrm{CN}$ with and without a magnetic field. The spectra of $\mathrm{CN}$ were recorded at the same day and under the same experimental conditions as the argon spectra that were used for calibration. The CN cell (see Sect. 4.3.4) was used to create the discharge. Many of the emission lines show variations under the influence of the magnetic field in the form of broadening, splitting, shift in line position or a change in intensity. A visual inspection of the 0-0 band of the Red System (around $1.1 \mu \mathrm{m}$ or $9100 \mathrm{~cm}^{-1}$ ) revealed a few lines with a clear splitting of about $0.05 \mathrm{~cm}^{-1}$. This is about half of the splitting observed for the three argon lines used for calibration. However, most lines do not show a clear splitting but rather a line broadening or a line shift. The latter can be an effect of blended lines. A stronger magnetic field might result in a larger line-splitting, both in laboratory and stellar spectra. For example, Shulyak et al. (2010) report strong magnetic fields of $0.27 \mathrm{~T}$ for three $\mathrm{M}$ dwarfs. A dedicated analysis needs to be conducted in the future to identify the $\mathrm{CN}$ lines that are most sensitive to the magnetic field and can be useful to probe the magnetic fields of cool stars.

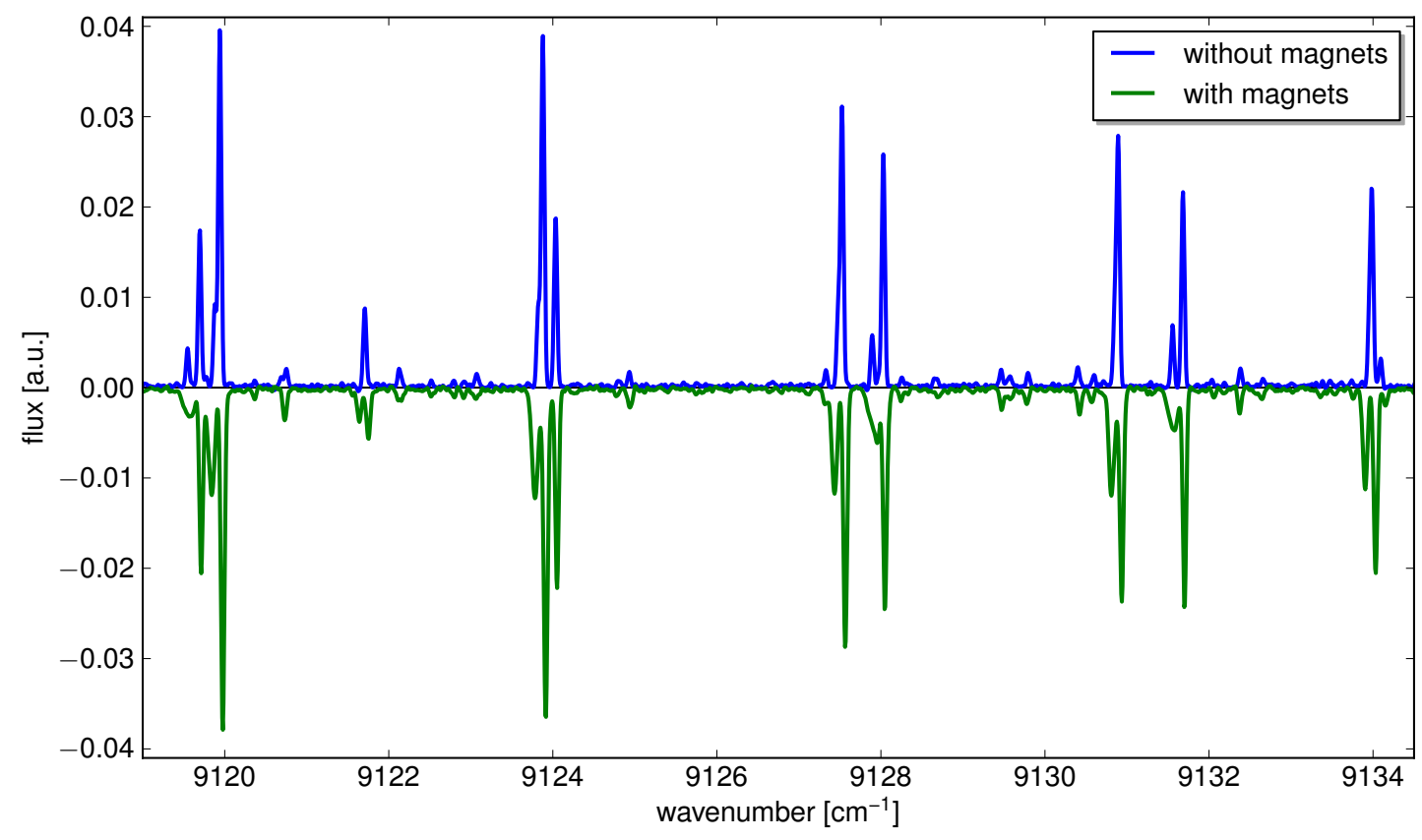

Figure 6.6: Emission spectrum of $C N$ molecules (lines from the 0-0 band of the Red System). The blue curve (top) shows the spectrum without a magnetic field. The green curve (folded to the lower half for better visibility) shows the spectrum with the magnets installed. 



\section{Summary and discussion}

Atomic and molecular emission spectra can be recorded from a microwave-induced plasma (MIP). An experimental setup with an MIP lamp has been constructed and spectra of gas discharges were recorded using a high-resolution Fourier transform spectrometer (FTS). Gas discharge spectra of molecular nitrogen and the cyano radical (CN) exhibit many emission lines in the near-infrared. The analysis of spectra from these species focused on the usability as wavelength standards for astrophysical spectrographs in the wavelength range between approximately $1 \mu \mathrm{m}$ and $2 \mu \mathrm{m}$. As summarized in Chap. 1, current wavelength calibration sources for high-precision spectroscopy in this spectral range are sparse or have not yet proven long-time reliability. It is therefore reasonable to investigate the potential of alternative calibration sources, such as molecular emission spectra from the MIP lamp.

Characteristics of the MIP lamp are the sealed gas cell and the electrodeless design. A sealed gas cell offers the best possibility to record spectra repeatedly under the same experimental conditions. This is important for wavelength calibration where a reproducible spectrum is a requirement. The proper cleaning and filling of the gas cell have proven to be crucial to avoid contamination in the spectrum from unwanted species. In addition, the sealed gas cell allows for a compact setup of the MIP lamp with no requirements for gas supply during operation. The mobility of the setup, even in the current experimental state, has been demonstrated with its transport from the laboratory in Göttingen to the ESO integration hall in Garching, and the subsequent successful operation of the MIP lamp in combination with the CRIRES spectrograph. Only minimal wear effects were originally expected because of the electrodeless operation, but only 180 hours of lifetime could be demonstrated for a discharge cell in the MIP lamp. The lifetime is probably limited by absorption of the gas by the inner surface of the glass tube. Thus, the MIP lamp does not yet achieve the lifetime of other emission sources, such as hollow cathode lamps (HCLs) which operate 4-6 times longer for typical operational currents (Kerber et al., 2007). Future advancements in the design of the gas cell (e.g., a larger gas reservoir) and a different mode of operation (e.g., less microwave power and shorter run-time at a stretch) might extend the lifetime.

The FTS at the Institut für Astrophysik in Göttingen was the primary instrument to record spectra of the MIP lamp. A computer script has been developed to determine the parameters of emission lines from the observed high-resolution spectra. It uses a superposition of Gaussian or Voigt profiles to fit the recorded data and is tailored to work with densely-spaced spectral lines over a large spectral range. For wavelength stability tests of the MIP lamp, time series of the discharges were recorded simultaneously with a gas absorption cell. The absorption cell serves as a relative wavelength reference to track drifts of the FTS wavelength scale. An existing algorithm was modified to disentangle the 
wavelength shifts of the two sources in the combined spectra. The software tools developed to analyze the spectra of the MIP lamp might also be beneficial when studying FTS spectra of other sources.

\subsection{Wavelength calibration}

In the context of wavelength calibration, I analyzed spectra of gas discharges using two different sealed gas cells. One gas cell, the $\mathrm{N}_{2}$ cell, was filled with pure nitrogen. The second cell, the CN cell, was filled with a gas mixture of nitrogen, carbon monoxide, and helium.

For the $\mathrm{N}_{2}$ cell, between approximately 40000 and 60000 emission lines could be detected in the spectral range $4500-11000 \mathrm{~cm}^{-1}(0.9-2.2 \mu \mathrm{m})$. The exact number of detected lines depends on the microwave (MW) power that was used to operate the discharge. For example, 51776 lines with intensities above the detection threshold were observed when the discharge was operated at $50 \mathrm{~W}$. The spectral lines of molecular nitrogen cover the entire spectral range under investigation. The spectrum of the $\mathrm{CN}$ cell exhibits additional vibrational bands from the $\mathrm{CN}$ molecule. About $4500 \mathrm{CN}$ lines were observed in the spectrum of the discharge operated at a MW power of $50 \mathrm{~W}$ (spectral range: $4500-10000 \mathrm{~cm}^{-1}$ ). However, the spectrum is dominated by the approximately 26000 lines from molecular nitrogen. The dynamic range of the detected lines covers about four orders of magnitude. The detection limit for spectral lines at low intensities is set by the noise level in the spectrum and by the wavelength-depended detection efficiency of the measuring system. A typical width of the narrow molecular emission lines is about $0.04 \mathrm{~cm}^{-1}$ at $\tilde{\nu}=10000 \mathrm{~cm}^{-1}(0.004 \mathrm{~nm}$ at $\lambda=1000 \mathrm{~nm})$.

Many of the emission lines will not be usable for wavelength calibration because of the inhomogeneities regarding line separations and peak intensities. In fact, many lines are overlapping even at the high resolution provided by the FTS $(R \approx 560000$ at $\lambda=1 \mu \mathrm{m})$. The percentage of blended lines is between $35 \%$ and $55 \%$ in the spectra recorded with the FTS, and these numbers will increase when a spectrum is recorded at a lower spectral resolution with an astrophysical spectrograph. Blends are a problem for wavelength calibration, because the apparent position of a blended spectral feature does not match the reference position of the corresponding unblended lines. This makes accurate determination of line positions more complicated. In addition, the shape of a blended line changes over time when the individual components do not have a constant relative intensity. It is very difficult to make a general statement concerning the number of usable lines that accounts for all possible characteristics of instruments (e.g., detector response) and wavelength ranges. In general, the molecular emission spectra are best used with high resolution spectrographs ( $R \approx 100000$ and higher) which resolve as many lines as possible. The highest number of spectral lines usable for wavelength calibration can be expected in the spectral ranges where strong vibrational bands are present (e.g., CN bands around $1.1 \mu \mathrm{m}$ and $1.4 \mu \mathrm{m}$, and $\mathrm{N}_{2}$ bands around $1.04 \mu \mathrm{m}$ and $1.2 \mu \mathrm{m}$ ). A calibration task that is less affected by blended lines is the measurement of relative wavelength shifts: a cross-correlation can be used to determine the wavelength shift of a stable reference spectrum, which determines the drift 
of the measuring system. In contrast to HCLs, the molecular emission spectra from the MIP lamp do not contain many strong atomic lines that might saturate the detector or contaminate parts of a simultaneously recorded science spectrum.

The wavelength stability of spectra of the $\mathrm{N}_{2}$ discharge cell and the CN discharge cell has been measured with a precision of about $1 \mathrm{~m} / \mathrm{s}$ over 24 hours using the spectral ranges $5600-6700 \mathrm{~cm}^{-1}$ and $7500-10000 \mathrm{~cm}^{-1}$. A very similar result has been obtained from measurements of the CN cell using the smaller spectral range $8700-9200 \mathrm{~cm}^{-1}$ which is dominated by the strong $0-0$ vibrational band of the CN molecule's Red System. This shows that the stability of about $1 \mathrm{~m} / \mathrm{s}$ is not only obtained for the molecular nitrogen lines, but also for the lines from the CN molecule and even in a smaller spectral range. The achievable precision of the RV stability measurements is limited by the spectral properties of the acetylene $\left(\mathrm{C}_{2} \mathrm{H}_{2}\right)$ absorption cell that serves as a relative wavelength reference. The absorption band of $\mathrm{C}_{2} \mathrm{H}_{2}$ has relatively few lines and therefore a lower $\mathrm{RV}$ information content compared to the discharge spectra. The measured RV stability on the $\mathrm{m} / \mathrm{s}$ level is comparable to the performance of gas absorption cells used for wavelength calibration in the near-infrared (e.g., Seifahrt \& Käufl, 2008, Bean et al., 2010). In the future, the wavelength stability might be measured with a higher precision if a laser frequency comb becomes available to externally calibrate the FTS. With a theoretical RV information content of better than $10 \mathrm{~cm} / \mathrm{s}$, the discharge spectra have the potential to become very precise (relative) references if the corresponding RV stability can be demonstrated. The wavelength stability tests have been carried out at a resolving power of $R \approx 150000$ which is in the range of future spectrographs such as the planed E-ELT/HIRES (Zerbi et al., 2014).

Aging behavior and wavelength stability are similar for the $\mathrm{N}_{2}$ cell and the CN cell, with no clear indication which discharge is more promising to be a reliable calibrator. In comparison with the pure nitrogen discharge, the $\mathrm{CN}$ cell provides additional vibrational bands from the $\mathrm{CN}$ molecule that can be useful for wavelength calibration, especially if the observable dynamic range is limited. For example, the strong vibrational band of $\mathrm{CN}$ at around $9000 \mathrm{~cm}^{-1}$ fills a gap between two bands of $\mathrm{N}_{2}$. However, the relative intensities of spectral lines from different species may evolve differently, as it is suspected from the results of the wavelength stability measurements of the $\mathrm{CN}$ cell. This might limit the precision of the calibration. In addition, the filling process of the $\mathrm{CN}$ cell with three gas components is rather complicated and the gas mixture is very difficult to reproduce with the same partial pressures. Therefore, I recommend using the pure nitrogen spectrum as a wavelength reference if the number of lines is sufficient in the spectral range of interest.

\subsection{Outlook}

Molecular emission spectra are an interesting alternative for wavelength calibration because of the large number of spectral lines and the high wavelength stability. The measurements with the CRIRES spectrograph have demonstrated that wavelength calibration is possible in a small spectral region with strong emission lines from CN. The small spectral coverage of the observation prevents a more extensive investigation. Comprehensive testing of the 
MIP lamp with an astrophysical spectrograph is now necessary to learn more about the performance and the usability of the MIP lamp in the field.

Some improvements to the MIP lamp need to be made for convenient and routine operation outside the laboratory. First, the size of the setup should be reduced to ensure better mobility. Without the $\mathrm{C}_{2} \mathrm{H}_{2}$ absorption cell, the setup can be designed in a compact way: a metal enclosure holds the MW cavity, a focusing lens and a fiber connector, and provides space for the discharge gas cell (compare Fig. 5.1). Second, a larger gas reservoir should be tested for the discharge gas cell to achieve a longer lifetime (compare Salit et al., 2004). Third, the air cooling permits a flexible usage of the MIP lamp because it requires pressurized air. An alternative could be a liquid-cooled discharge cell as described by Salit et al. (2004). They work with a quartz cell that has an inner diameter of $7 \mathrm{~mm}$ and is surrounded by a water jacket for temperature stabilization. Salit et al. (2004) used water or a solution of ethylene glycol mixed with ethanol as coolants. Fourth, the ignition process needs to be automated. Principally, it should be possible to operate the MW generator remotely using the serial connection and a computer script, and to synchronize the plasma ignition with the high-voltage generator.

The MIP lamp can be operated with discharge tubes filled with a variety of gases and this allows the analysis of emission spectra from different species that are of astrophysical interest. In Chap. 6, first measurements with the MIP lamp in a magnetic field were presented. Two ring magnets were installed on both sides of the MW cavity axially symmetric with the optical axis. Using the Zeeman splitting of three argon lines in a discharge spectrum, a magnetic flux density of $B=(0.130 \pm 0.003) \mathrm{T}$ could be determined. As already discussed in Sect. 6.4, future work needs to focus on a stronger, more homogeneous field and a more accurate calibration. Then, the next step could be the analysis of molecular spectra. For example, the CN molecule has been observed in sunspots, and Berdyugina \& Solanki (2002) claim that its Red System could be a sensitive tool for solar and stellar magnetic studies. A first measurement of the CN cell with the installed magnets shows that many of the CN lines change through broadening, splitting, shift in line position or a change in intensity. A detailed investigation is needed to identify the $\mathrm{CN}$ lines that are most sensitive to the magnetic field and to determine Landé factors. 


\section{Bibliography}

Abrams, M. C., Davis, S. P., Rao, M. L. P., Engleman, Jr., R. \& Brault, J. W. (1994) High-resolution Fourier transform spectroscopy of the Meinel system of OH. ApJS, 93, 351-395.

Akatsuka, H. (2012) Progresses in Experimental Study of $\mathrm{N}_{2}$ Plasma Diagnostics by Optical Emission Spectroscopy. In Chemical Kinetics, edited by V. Patel, InTech.

Anglada-Escudé, G. \& Butler, R. P. (2012) The HARPS-TERRA Project. I. Description of the Algorithms, Performance, and New Measurements on a Few Remarkable Stars Observed by HARPS. ApJS, 200, 15-33.

Anglada-Escudé, G., Plavchan, P., Mills, S., Gao, P., García-Berríos, E., Lewis, N. S., Sung, K., Ciardi, D. et Al. (2012) Design and Construction of Absorption Cells for Precision Radial Velocities in the K Band Using Methane Isotopologues. PASP, 124, 586-597.

Appenzeller, I. (2013) Introduction to Astronomical Spectroscopy. Cambridge University Press.

Baranne, A., Mayor, M. \& Poncet, J. L. (1979) CORAVEL - A new tool for radial velocity measurements. Vistas in Astronomy, 23, 279-316.

Baranne, A., Queloz, D., Mayor, M., Adrianzyk, G., Knispel, G., Kohler, D., Lacroix, D., Meunier, J.-P. ET AL. (1996) ELODIE: A spectrograph for accurate

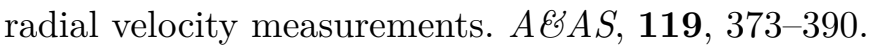

Barge, P., Baglin, A., Auvergne, M., Rauer, H., Léger, A., Schneider, J., Pont, F., Aigrain, S. ET AL. (2008) Transiting exoplanets from the CoRoT space mission. I. CoRoT-Exo-1b: a low-density short-period planet around a G0V star. $A \mathscr{E} A$, 482, L17-L20.

Bean, J. L., Seifahrt, A., Hartman, H., Nilsson, H., Wiedemann, G., Reiners, A., Dreizler, S. \& Henry, T. J. (2010) The CRIRES Search for Planets Around the Lowest-mass Stars. I. High-precision Near-infrared Radial Velocities with an Ammonia Gas Cell. ApJ, 713, 410-422.

Berdyugina, S. V. \& Solanki, S. K. (2002) The molecular Zeeman effect and diagnostics of solar and stellar magnetic fields. I. Theoretical spectral patterns in the Zeeman regime. $A \mathscr{E A}, \mathbf{3 8 5}, 701-715$.

Bernath, P. F. (2005) Spectra of Atoms and Molecules. Oxford University Press. 
Boesch, A. \& Reiners, A. (2015) Spectral line lists of a nitrogen gas discharge for wavelength calibration in the range $4500-11000 \mathrm{~cm}^{-1}$. A $A \& A, \mathbf{5 8 2}, \mathrm{A} 43$.

Boesch, A., Reiners, A., Bernath, P. F. \& Seifahrt, A. (2014) Near-infrared wavelength calibration of astrophysical spectrographs with the emission spectrum of the CN molecule. In SPIE Conference Series, vol. 9147.

Borucki, W. J., Koch, D. G., Brown, T. M., Basri, G., Batalha, N. M., Caldwell, D. A., Cochran, W. D., Dunham, E. W. et al. (2010) Kepler-4b: A Hot Neptune-like Planet of a G0 Star Near Main-sequence Turnoff. ApJ, 713, L126-L130.

Bouchy, F., Pepe, F. \& Queloz, D. (2001) Fundamental photon noise limit to radial velocity measurements. $A \mathscr{E} A, \mathbf{3 7 4}, 733-739$.

Bouchy, F. \& Queloz, D. (2007) Radial Velocity Follow-up for Confirmation and Characterization of Transiting Exoplanets. In Transiting Extrasolar Planets Workshop, edited by C. Afonso, D. Weldrake \& T. Henning, vol. 366 of Astronomical Society of the Pacific Conference Series.

Brault, J. W. (1985) Fourier Transform Spectroscopy. In High Resolution in Astronomy, edited by A. O. Benz, M. Huber \& M. Mayor, Geneva Observatory.

Brooke, J. S. A., Ram, R. S., Western, C. M., Li, G., Schwenke, D. W. \& Bernath, P. F. (2014) Einstein A Coefficients and Oscillator Strengths for the $A^{2} \Pi-$ $\mathrm{X}^{2} \Sigma^{+}$(Red) and $\mathrm{B}^{2} \Sigma^{+}-\mathrm{X}^{2} \Sigma^{+}$(Violet) Systems and Rovibrational Transitions in the $\mathrm{X}^{2} \Sigma^{+}$State of CN. ApJS, 210, 23-37.

Browning, R. C., Baker, E. A., Herron, J. A. \& Kram, R. (2006) Effects of obesity and sex on the energetic cost and preferred speed of walking. Journal of Applied Physiology, 100, 390-398.

Butler, R. P., Marcy, G. W., Williams, E., McCarthy, C., Dosanjh, P. \& Vogt, S. S. (1996) Attaining Doppler Precision of $3 \mathrm{~m} \mathrm{~s}^{-1}$. PASP, 108, 500-509.

Campbell, B. \& Walker, G. A. H. (1979) Precision radial velocities with an absorption cell. PASP, 91, 540-545.

Campbell, P. (1994) Permanent magnet materials and their application. Cambridge University Press.

Chamberlain, J. (1979) The Principles of Interferometric Spectroscopy. Wiley.

Cobine, J. D. \& Wilbur, D. A. (1951) The Electronic Torch and Related High Frequency Phenomena. Journal of Applied Physics, 22(6), 835-841.

Connes, P. (1985) Absolute astronomical accelerometry. ApESSS, 110, 211-255.

Crozet, P., Dobrev, G., Richard, C. \& Ross, A. J. (2014) Determination of Landé factors in the $\mathrm{F}^{4} \Delta_{5 / 2,7 / 2}$ state of ${ }^{56} \mathrm{FeH}$ by laser excitation spectroscopy. Journal of Molecular Spectroscopy, 303, 46-53. 
Crozet, P., Tourasse, G., Ross, A., Paletou, F. \& López Ariste, A. (2012) Laboratory determination of Landé factors for the molecular radical FeH. In European Conference on Laboratory Astrophysics - ECLA, vol. 58 of EAS Publications Series.

Davis, P. D., Abrams, M. C. \& Brault, J. W. (2001) Fourier Transform Spectrometry. Academic Press.

DemtröDer, W. (2006) Experimentalphysik 2: Elektrizität und Optik. Springer.

Donati, J.-F. \& Landstreet, J. D. (2009) Magnetic Fields of Nondegenerate Stars. $A R A \& A, 47,333-370$.

Drosg, M. (2009) Dealing with Uncertainties. Springer.

Effantin, C., Amiot, C. \& Verges, J. (1979) Analysis of the (0-0), (1-0), and (2-0) bands of the $\mathrm{B}^{3} \Pi_{g} \rightarrow \mathrm{A}^{3} \Sigma_{u}^{+}$system of ${ }^{14} \mathrm{~N}_{2}$ and ${ }^{15} \mathrm{~N}_{2}$. Journal of Molecular Spectroscopy, 76, 221-265.

Elghazaly, M. H., Solyman, S. \& Abdel baky, A. M. (2007) Study of Some Basic Transport Coefficients in Noble-Gas Discharge Plasmas. Egypt. J. Solids, 30, 137-149.

Fehsenfeld, F. C., Evenson, K. M. \& Broida, H. P. (1965) Microwave Discharge Cavities Operating at $2450 \mathrm{MHz}$. Review of Scientific Instruments, 36(3), 294-298.

Ferguson, D. W., Narahari Rao, K., Martin, P. A. \& Guelachvili, G. (1992) High resolution infrared Fourier transform emission spectra of the ${ }^{14} \mathrm{~N}_{2}^{+}$Meinel system: $\mathrm{A}^{2} \Pi_{u}-\mathrm{X}^{2} \Sigma_{g}^{+}$. Journal of Molecular Spectroscopy, 153, 599-609.

Figueira, P., Pepe, F., Melo, C. H. F., Santos, N. C., Lovis, C., Mayor, M., Queloz, D., Smette, A. et Al. (2010) Radial velocities with CRIRES. Pushing precision down to $5-10 \mathrm{~m} / \mathrm{s}$. A\& $A, \mathbf{5 1 1}$, A 55 .

Follert, R., Dorn, R. J., Oliva, E., Lizon, J. L., Hatzes, A., Piskunov, N., Reiners, A., Seemann, U. ET AL. (2014) CRIRES+: a cross-dispersed high-resolution infrared spectrograph for the ESO VLT. In SPIE Conference Series, vol. 9147.

Fraden, J. (2010) Handbook of Modern Sensors: Physics, Designs, and Applications. Springer.

Fray, N., Bénilan, Y., Cottin, H., Gazeau, M.-C. \& Crovisier, J. (2005) The origin of the CN radical in comets: A review from observations and models. Planet. Space Sci., 53, 1243-1262.

Gilbert, S. L. \& Swann, W. C. (2001) Acetylene ${ }^{12} C_{2} H_{2}$ Absorption Reference for $1510 \mathrm{~nm}$ to $1540 \mathrm{~nm}$ Wavelength Calibration-SRM 2517a. Tech. Rep. 260-133, NIST.

Gilmore, F. R., Laher, R. R. \& Espy, P. J. (1992) Franck-Condon Factors, r-Centroids, Electronic Transition Moments, and Einstein Coefficients for Many Nitrogen and Oxygen Band Systems. J. Phys. Chem. Ref. Data, 21, 1005-1107. 
Goodman, J. \& Weare, J. (2010) Ensemble samplers with affine invariance. Comm. App. Math. and Conp. Sci., 5(1), 65-80.

Griffin, R. \& GRIFFIn, R. (1973) On the possibility of determining stellar radial velocities to $0.01 \mathrm{~km} \mathrm{~s}^{-1}$. MNRAS, 162, 243-253.

Griffiths, P. R. \& DE Haseth, J. A. (2007) Fourier Transform Infrared Spectrometry. Wiley.

Grigorian, G. \& Cenian, A. (2011) Formation and Excitation of CN Molecules in HeCO- $\mathrm{N}_{2}-\mathrm{O}_{2}$ Discharge Plasmas. Plasma Chemistry and Plasma Processing, 31, 337-352.

Haken, H. \& Wolf, H. C. (2004) Atom- und Quantenphysik. Springer.

Harvey, J. W. (1973) Polarization of Red System CN Lines in Sunspots. Sol. Phys., 28, $43-47$.

Heacox, W. D. (1986) On the application of optical-fiber image scramblers to astronomical spectroscopy. AJ, 92, 219-229.

Hearnshaw, J. B. (2014) The Analysis of Starlight: Two Centuries of Astronomical Spectroscopy. Cambridge University Press.

Hilditch, R. W. (2001) An Introduction to Close Binary Stars. Cambridge University Press.

Hinkle, K., Wallace, L. \& Livingston, W. (1995) Infrared Atlas of the Arcturus Spectrum, 0.9-5.3 microns. PASP, 107, 1042-1046.

Hinkle, K. H., Joyce, R. R., Hedden, A., Wallace, L. \& Engleman, JR., R. (2001) Wavelength Calibration of Near-Infrared Spectra. PASP, 113, 548-566.

Hirao, T., Pinchemel, B. \& Bernath, P. F. (2000) The Rotational Analysis of the $\mathrm{A}^{2} \Pi_{r}-\mathrm{X}^{2} \Sigma^{+}$Band System of MgBr. Journal of Molecular Spectroscopy, 202, 213-222.

Huke, P., Holzhüter, H. \& Reiners, A. (2015) Transferring the Rb+ hyperfinestructure stability to a Fabry-Perot resonator used as a frequency standard for astronomical spectrographs. In SPIE Conference Series, vol. 9526.

IlLiNG, R. M. E. (1981) Broad-band polarization in molecular spectra. ApJ, 248, 358-372.

Jankowski, K. J. \& Reszke, E. (2011) Microwave Induced Plasma Analytical Spectrometry. The Royal Society of Chemistry.

Kang, K. R. (1999) Astrophysical Formulae, vol. I: Radiation, Gas Processes and High Energy Astrophysics. Springer.

Karttunen, H., Kroeger, P., Oja, H., Poutanen, M. \& Donner, K. J. (2003) Fundamental astronomy. Springer. 
Käufl, H.-U., Ballester, P., Biereichel, P., Delabre, B., Donaldson, R., Dorn, R., Fedrigo, E., Finger, G. ET AL. (2004) CRIRES: a high-resolution infrared spectrograph for ESO's VLT. In SPIE Conference Series, vol. 5492.

Kauppinen, J. \& Partanen, J. (2001) Fourier Transforms in Spectroscopy. WILEY-VCH.

Kerber, F., Nave, G. \& Sansonetti, C. J. (2008) The Spectrum of Th-Ar Hollow Cathode Lamps in the 691-5804 nm region: Establishing Wavelength Standards for the Calibration of Infrared Spectrographs. ApJS, 178, 374-381.

Kerber, F., Nave, G., Sansonetti, C. J., Bristow, P. \& Rosa, M. R. (2007) The Spectrum of Th-Ar Hollow Cathode Lamps in the 900-4500 nm Region: Establishing Wavelength Standards for the Calibration of VLT Spectrographs. In The Future of Photometric, Spectrophotometric and Polarimetric Standardization, edited by C. Sterken, vol. 364 of Astronomical Society of the Pacific Conference Series.

Kirkbright, G. F. \& SArgent, M. (1974) Atomic absorption and fluorescence spectroscopy. Academic Press.

Kitchin, C. (2012) Exoplanets: Finding, Exploring and Understanding Alien Worlds. Springer.

Koch, O. G. (1985) Analytische Chemie des Mangans. Springer.

Kramida, A., Yu. Ralchenko, Reader, J. \& And NiST ASD Team (2014) NIST Atomic Spectra Database (ver. 5.2), [Online]. Available: http://physics.nist.gov/asd. National Institute of Standards and Technology, Gaithersburg, MD.

Kurucz, R. L., Furenlid, I., Brault, J. \& Testerman, L. (1984) Solar flux atlas from 296 to $1300 \mathrm{~nm}$. Tech. Rep., NSO Atlas No. 1.

Laher, R. R. \& Gilmore, F. R. (1991) Impoved Fits for the Vibrational and Rotational Constants of Many States of Nitrogen and Oxygen. J. Phys. Chem. Ref. Data, 20, 685-693.

Lambert, D. L., Brown, J. A., Hinkle, K. H. \& Johnson, H. R. (1984) Carbon, nitrogen, and oxygen abundances in Betelgeuse. ApJ, 284, 223-237.

Landi Degl'Innocenti, E. (1982) On the effective Landé factor of magnetic lines. Sol. Phys., 77, 285-289.

Lide, D. R. (ed.) (2006) CRC handbook of chemistry and physics. Taylor \& Francis.

Lofthus, A. \& Krupenie, P. H. (1977) The Spectrum of Molecular Nitrogen. J. Phys. Chem. Ref. Data, 6, 113-307.

Lovis, C. \& Fischer, D. (2010) Radial Velocity Techniques for Exoplanets. In Exoplanets, edited by S. Seager, University of Arizona Press, pp. 27-53.

Lovis, C. \& PePe, F. (2007) A new list of thorium and argon spectral lines in the visible. $A \mathscr{E} A, 468,1115-1121$. 
Lu, W. \& Rucinski, S. M. (1999) Radial Velocity Studies of Close Binary Stars. I. AJ, $118,515-526$.

Mahadevan, S. \& Ge, J. (2009) The Use of Absorption Cells as a Wavelength Reference for Precision Radial Velocity Measurements in the Near-Infrared. ApJ, 692, 1590-1596.

Mahadevan, S., Ramsey, L., Bender, C., Terrien, R., Wright, J. T., Halverson, S., Hearty, F., Nelson, M. et Al. (2012) The habitable-zone planet finder: a stabilized fiber-fed NIR spectrograph for the Hobby-Eberly Telescope. In SPIE Conference Series, vol. 8446.

Marcy, G. W. \& Butler, R. P. (1992) Precision radial velocities with an iodine absorption cell. PASP, 104, 270-277.

Marcy, G. W. \& Butler, R. P. (1996) A Planetary Companion to 70 Virginis. ApJ, 464, L147-L151.

Mayor, M., Pepe, F., Queloz, D., Bouchy, F., Rupprecht, G., Lo Curto, G., Avila, G., Benz, W. et Al. (2003) Setting New Standards with HARPS. The Messenger, 114, 20-24.

Mayor, M. \& Queloz, D. (1995) A Jupiter-mass companion to a solar-type star. Nature, 378, 355-359.

Meyer, D. M. \& JuRA, M. (1985) A precise measurement of the cosmic microwave background temperature from optical observations of interstellar CN. ApJ, 297, 119-132.

Murphy, M. T., Udem, T., Holzwarth, R., Sizmann, A., Pasquini, L., AraujoHauck, C., Dekker, H., D'Odorico, S. et Al. (2007) High-precision wavelength calibration of astronomical spectrographs with laser frequency combs. $M N R A S, \mathbf{3 8 0}$, 839-847.

Nave, G., Sansonetti, C. J., Penton, S. V., Cunningham, N., Beasley, M., Osterman, S., Kerber, F., Keyes, C. D. . et Al. (2012) Lifetime and Failure Characteristics of Pt/Ne Hollow Cathode Lamps Used as Calibration Sources for UV Space Instruments. PASP, 124, 1295-1308.

Nieva, M.-F., Przybilla, N., Seifahrt, A., Butler, K., Käufl, H.-U. \& Kaufer, A. (2011) Near-IR spectroscopy of OB stars with VLT/CRIRES. Bulletin de la Societe Royale des Sciences de Liege, 80, 175-179.

Norlén, G. (1973) Wavelengths and Energy Levels of Ar I and Ar II based on New Interferometric Measurements in the Region 3400-9800 A. Phys. Scr, 8, 249-268.

Olivero, J. J. \& Longbothum, R. L. (1977) Empirical fits to the Voigt line width: A brief review. J. Quant. Spectrosc. Radiat. Transfer, 17(2), 233-236.

Osterbrock, D. E., Fulbright, J. P. \& Bida, T. A. (1997) Night-Sky High-Resolution Spectral Atlas of OH Emission Lines for Echelle Spectrograph Wavelength Calibration. II. PASP, 109, 614-627. 
Osterbrock, D. E., Fulbright, J. P., Martel, A. R., Keane, M. J., Trager, S. C. \& BAsRI, G. (1996) Night-Sky High-Resolution Spectral Atlas of $\mathrm{OH}$ and $\mathrm{O}_{2}$ Emission Lines for Echelle Spectrograph Wavelength Calibration. PASP, 108, 277-308.

Palmer, B. A. \& Engleman, R. (1983) Atlas of the Thorium spectrum. Los Alamos: National Laboratory.

Parimucha, Š. \& ŠKodA, P. (2007) Comparison of Selected Methods for Radial Velocity Measurements. In IAU Symposium, edited by W. I. Hartkopf, P. Harmanec \& E. F. Guinan, vol. 240.

Pepe, F. \& Lovis, C. (2008) High Resolution and High Precision-Spectroscopy with HARPS. In 2007 ESO Instrument Calibration Workshop, edited by A. Kaufer \& F. Kerber.

Pepe, F., Mayor, M., Galland, F., Naef, D., Queloz, D., Santos, N. C., Udry, S. \& Burnet, M. (2002) The CORALIE survey for southern extra-solar planets VII. Two short-period Saturnian companions to HD 108147 and HD 168746. A\& A, 388, 632-638.

Perryman, M. (2011) The Exoplanet Handbook. Cambridge University Press.

Perryman, M. (2012) The History of Exoplanet Detection. Astrobiology, 12, 928-939.

Pozar, D. M. (2012) Microwave engineering. Wiley.

Press, W. H., Teukolsky, S. A., Vetterling, W. T. \& Flannery, B. P. (2002) Numerical recipes in $\mathrm{C}++$ : the art of scientific computing. Cambridge University Press.

Queloz, D. (1995) Echelle Spectroscopy with a CCD at Low Signal-To-Noise Ratio. In New Developments in Array Technology and Applications, edited by A. G. D. Philip, K. Janes \& A. R. Upgren, vol. 167 of IAU Symposium.

Quirrenbach, A., Amado, P. J., Caballero, J. A., Mundt, R., Reiners, A., Ribas, I., Seifert, W., Abril, M. et Al. (2014) CARMENES instrument overview. In SPIE Conference Series, vol. 9147.

Ram, R. S., Wallace, L. \& Bernath, P. F. (2010) High resolution emission spectroscopy of the $\mathrm{A}^{2} \Pi-\mathrm{X}^{2} \Sigma^{+}$(red) system of ${ }^{12} \mathrm{C}^{14} \mathrm{~N}$. Journal of Molecular Spectroscopy, 263, 82-88.

Ramírez, I. \& Allende Prieto, C. (2011) Fundamental Parameters and Chemical Composition of Arcturus. ApJ, 743, 135.

Redman, S. L., Kerber, F., Nave, G., Mahadevan, S., Ramsey, L. W., Smoker, J., KäUft, H.-U. \& Figueira, P. R. L. (2012) Near-infrared calibration systems for precise radial-velocity measurements. In SPIE Conference Series, vol. 8446.

Redman, S. L., Lawler, J. E., Nave, G., Ramsey, L. W. \& Mahadevan, S. (2011) The Infrared Spectrum of Uranium Hollow Cathode Lamps from $850 \mathrm{~nm}$ to $4000 \mathrm{~nm}$ : Wavenumbers and Line Identifications from Fourier Transform Spectra. ApJS, 195, 24. 
Redman, S. L., Nave, G. \& Sansonetti, C. J. (2014) The Spectrum of Thorium from $250 \mathrm{~nm}$ to $5500 \mathrm{~nm}$ : Ritz Wavelengths and Optimized Energy Levels. ApJS, 211, 4.

Regan, R. J. \& HaugsjaA, P. O. (1977) Tunable Microwave Cavity. US Patent No. 4002943.

Reiners, A., Bean, J. L., Huber, K. F., Dreizler, S., Seifahrt, A. \& Czesla, S. (2010) Detecting Planets Around Very Low Mass Stars with the Radial Velocity Method. ApJ, 710, 432-443.

Rothman, L. S., Gordon, I. E., Babikov, Y., Barbe, A., Chris Benner, D., Bernath, P. F., Birk, M., Bizzocchi, L. Et Al. (2013) The HITRAN2012 molecular spectroscopic database. J. Quant. Spec. Radiat. Transf., 130, 4-50.

Rousselot, P., Lidman, C., Cuby, J.-G., Moreels, G. \& Monnet, G. (2000) Night-sky spectral atlas of $\mathrm{OH}$ emission lines in the near-infrared. A $\mathcal{E} A, \mathbf{3 5 4}, 1134-1150$.

Roux, F., Michaud, F. \& Verges, J. (1983) High-resolution Fourier spectrometry of ${ }^{14} \mathrm{~N}_{2}$ infrared emission spectrum: Extensive analysis of the $\mathrm{B}^{3} \Pi_{g}-\mathrm{A}^{3} \Sigma_{u}{ }^{+}$system. Journal of Molecular Spectroscopy, 97, 253-265.

Rutscher, A. (2001) Characteristics of low-temperature plasmas under non-thermal conditions - a short summary. In Low Temperature Plasma Physics, edited by R. Hippler, S. Pfau, M. Schmidt \& K. H. Schoenbach, Wiley-VCH.

Salit, M. L., Sansonetti, C. J., Veza, D. \& Travis, J. C. (2004) Investigation of single-factor calibration of the wave-number scale in Fourier-transform spectroscopy. Journal of the Optical Society of America B Optical Physics, 21, 1543-1550.

Santos, N. C., Pasquini, L., Correia, A. C. M. \& Romaniello, M. (eds.) (2008) Precision Spectroscopy in Astrophysics, Proceedings of the ESO/Lisbon/Aveiro Conference held in Aveiro, Portugal, 11-15 September 2006.

Schäfer, S. \& Reiners, A. (2012) Two Fabry-Perot interferometers for high precision wavelength calibration in the near-infrared. In SPIE Conference Series, vol. 8446.

Schmidt, M. \& Conrads, H. (2001) Plasma sources. In Low Temperature Plasma Physics, edited by R. Hippler, S. Pfau, M. Schmidt \& K. H. Schoenbach, Wiley-VCH.

Seager, S. (2010) Exoplanets. University of Arizona Press.

Seemann, U., Anglada-Escudé, G., Bande, D., Bristow, P., Dorn, R. J., Follert, R., Gojak, D., Grunhut, J. ET AL. (2014) Wavelength calibration from $1-5 \mu \mathrm{m}$ for the CRIRES+ high-resolution spectrograph at the VLT. In SPIE Conference Series, vol. 9147.

Seifahrt, A. \& KÄUfl, H. U. (2008) High precision radial velocity measurements in the infrared. A first assessment of the RV stability of CRIRES. AESA, 491, 929-939. 
Shulyak, D., Reiners, A., Wende, S., Kochukhov, O., Piskunov, N. \& Seifahrt, A. (2010) Modelling the molecular Zeeman-effect in M-dwarfs: methods and first results. $A \& A, \mathbf{5 2 3}, \mathrm{A} 37$.

Skogerboe, R. K. \& Coleman, G. N. (1976) Microwave Plasma Emission Spectrometry. Analytical Chemistry, 48(7), 611-622.

Steinmetz, T., Wilken, T., Araujo-Hauck, C., Holzwarth, R., Hänsch, T. W. \& UDEM, T. (2009) Fabry-Pérot filter cavities for wide-spaced frequency combs with large spectral bandwidth. Applied Physics B: Lasers and Optics, 96, 251-256.

Swann, W. C. \& Gilbert, S. L. (2000) Pressure-induced shift and broadening of 1510-1540-nm acetylene wavelength calibration lines. Journal of the Optical Society of America B Optical Physics, 17, 1263-1270.

Takeda, Y., Ohshima, O., Kambe, E., Toda, H., Koyano, H., Sato, B., Nakamura, Y., NARItA, N. ET AL. (2015) Solar rotation inferred from radial velocities of the Sunas-a-star during the 2012 May 21 eclipse. PASJ, 67, 10.

Tennyson, J. (2011) Astronomical Spectroscopy. World Scientific Publishing.

Thibault, S., Rabou, P., Donati, J.-F., Desaulniers, P., Dallaire, X., Artigau, E., Pepe, F., Micheau, Y. Et Al. (2012) SPIRou @ CFHT: spectrograph optical design. In SPIE Conference Series, vol. 8446.

Thorne, A., Litzén, U. \& Johansson, S. (1999) Spectrophysics: Principles and Applications. Springer.

Tschöpel, P. (1979) Plasma excitation in spectrochemical analysis. In Comprehensive analytical chemistry, edited by G. Svehla, vol. 9, Elsevier, p. 173.

Udem, T., Holzwarth, R. \& Hänsch, T. W. (2002) Optical frequency metrology. Nature, 416, 233-237.

Valdivielso, L., Esparza, P., Martín, E. L., Maukonen, D. \& Peale, R. E. (2010) A New Gas Cell for High-precision Doppler Measurements in the Near-infrared. ApJ, 715, 1366-1369.

Valenti, J. A., Johns-Krull, C. M. \& Piskunov, N. E. (2001) Using FeH to Measure Magnetic Fields on Cool Stars and Brown Dwarfs. In 11th Cambridge Workshop on Cool Stars, Stellar Systems and the Sun, edited by R. J. Garcia Lopez, R. Rebolo \& M. R. Zapaterio Osorio, vol. 223 of Astronomical Society of the Pacific Conference Series.

Vallon, R., Ashworth, S. H., Crozet, P., Field, R. W., Forthomme, D., Harker, H., Richard, C. \& Ross, A. J. (2009) Room-Temperature Metal-Hydride Discharge Source, with Observations on $\mathrm{NiH}$ and FeH. Journal of Physical Chemistry A, 113, 13159-13166.

Voigtman, E. \& Winefordner, J. D. (1987) The multiplex disadvantage and excess low-frequency noise. Applied Spectroscopy, 41, 1182-1184. 
Wallace, L., Livingston, W. C., Bernath, P. F. \& Ram, R. S. (1998) An Atlas of the Sunspot Umbral Spectrum in the Red and Infrared from 1900 to $15050 \mathrm{~cm}^{-1}$ (6642 to $11230 \AA$ ). Technical report, N.S.O.

WARTEWig, S. (2003) IR and Raman spectroscopy: fundamental processing. Wiley-VCH.

Wilken, T., Lo Curto, G., Probst, R. A., Steinmetz, T., Manescau, A., Pasquini, L., González Hernández, J. I., Rebolo, R. et Al. (2012) A spectrograph for exoplanet observations calibrated at the centimetre-per-second level. Nature, 485, 611614.

Wilken, T., Lovis, C., Manescau, A., Steinmetz, T., Pasquini, L., Lo Curto, G., Hänsch, T. W., Holzwarth, R. ET AL. (2010) High-precision calibration of spectrographs using laser frequency combs. In SPIE Conference Series, vol. 7735.

Woan, G. (2000) The Cambridge Handbook of Physics Formulas. Cambridge University Press.

Wolszczan, A. \& Frail, D. A. (1992) A planetary system around the millisecond pulsar PSR1257 + 12. Nature, 355, 145-147.

Ycas, G. G., Quinlan, F., Diddams, S. A., Osterman, S., Mahadevan, S., Redman, S., Terrien, R., Ramsey, L. ET AL. (2012) Demonstration of on-sky calibration of astronomical spectra using a $25 \mathrm{GHz}$ near-IR laser frequency comb. Optics Express, 20, 6631.

Zeeman, P. (1897) The Effect of Magnetisation on the Nature of Light Emitted by a Substance. Nature, 55, 347.

Zerbi, F. M., Bouchy, F., Fynbo, J., Maiolino, R., Piskunov, N., Rebolo Lopez, R., Santos, N., Strassmeier, K. et Al. (2014) HIRES: the high resolution spectrograph for the E-ELT. In SPIE Conference Series, vol. 9147. 


\section{Acknowledgements}

First, I would like to thank my supervisor Ansgar Reiners for his support and advice. Ansgar, I appreciate very much that you always took the time to discuss my work and enabled me to develop the experiment.

I thank the additional members of my advisory committee from the Research Training Group "Extrasolar Planets and their Host Stars", Rick Hessman and Andreas Schweitzer, for regular discussions which helped to structure and advance the work. Many thanks to Thomas Giesen who volunteered to review this thesis as a second referee.

At the beginning of the $\mathrm{PhD}$ project I was given the opportunity to visit the research group of Peter Bernath and Michael Dulick at the Old Dominion University in Norfolk. They provided me with much practical information on Fourier transform spectroscopy and the microwave-induced plasma.

In Göttingen, I could use laboratory equipment from the Department of Physical Chemistry to fill the gas cells with support from Ulrich Schmitt. Peter Jeep and the team from the mechanical workshop fabricated parts of the experimental setup and helped with hardware issues.

I am thankful for the positive working atmosphere at the Institute. For all help related to the FTS and to experimental challenges, I would like to thank the team working in the optics laboratory, especially Ulrike Lemke, Ulf Seemann, Sebastian Schäfer, and Philipp Huke. A special thanks goes to Luis Fernando Sarmiento who shared the office with me for over three years. Luis, I will miss our discussions about science and beyond.

I would like to thank the following people who volunteered to proof-read individual parts of this thesis: Philipp Huke, Ulf Seemann, Denis Shulyak and Bill Galic.

Last but not least, I thank my family and friends for their support. Maren, your encouragement, understanding and love over the last years have been amazing. 